Supporting Information

\title{
Catalytic Enantioselective Synthesis of 1,4-Benzodioxepines
}

\author{
Xi Zou, ${ }^{a}$ Guangwu Sun, ${ }^{a}$ Hai Huang, ${ }^{\mathrm{c}}$ Jinping Wang*a, \\ Wen Yang*a and Jianwei Sun ${ }^{* b}$ \\ ${ }^{a}$ College of Chemistry and Chemical Engineering, Hunan University, Changsha 410082, \\ China \\ ${ }^{b}$ Department of Chemistry and Shenzhen Research Institute, The Hong Kong University \\ of Science and Technology, Clear Water Bay, Kowloon, Hong Kong SAR, China \\ c Jiangsu Key Laboratory of Advanced Catalytic Materials E Technology, School of \\ Petrochemical Engineering, Changzhou University, Changzhou 213164, China
}

\section{Table of Contents}

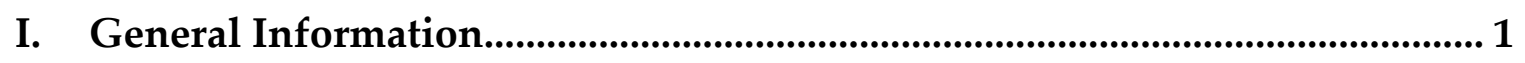

II. Synthesis of the Oxetane Substrates .......................................................... 3

III. Organocatalytic Intramolecular Desymmetrization of Oxetanes................. 22

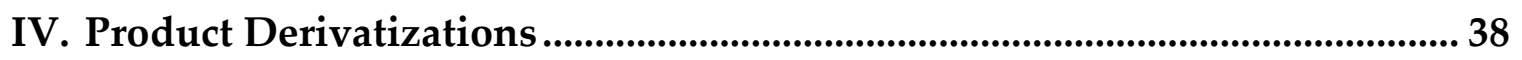

V. Determination of the Absolute Stereochemistry ........................................ 47

NMR Spectra and HPLC Traces 


\section{General Information}

Flash column chromatography was performed over silica gel (200-300 mesh) purchased from Qindao Puke Co., China. All air or moisture sensitive reactions were conducted in oven-dried glassware under nitrogen atmosphere using anhydrous solvents. Anhydrous dichloromethane, tetrahydrofuran, $\mathrm{N}, \mathrm{N}-$ dimethylformamide, and acetonitrile were purchased from Energy Chemical and used as received. ACS grade toluene, and ethyl ether were purchased from Sinopharm Chemical Reagent Co.,Ltd and used as received. ${ }^{1} \mathrm{H}$ and ${ }^{13} \mathrm{C}$ NMR spectra were collected on a Varian INOVA-400 NMR spectrometer using peaks of deuterated solvents as an internal standard ( ${ }^{1} \mathrm{H}$ NMR: $\mathrm{CDCl}_{3}$ at $7.26 \mathrm{ppm}$, d6acetone at $2.05 \mathrm{ppm} ;{ }^{13} \mathrm{C} \mathrm{NMR:} \mathrm{CDCl}_{3}$ at $77.0 \mathrm{ppm}$, d6-acetone at $\left.29.8 \mathrm{ppm}\right)$. Mass spectra were collected on a Thermo Scientific DSQ II mass spectrometer, a MALDI Micro MX mass spectrometer, or an API QSTAR XL System. Optical rotations were measured on JASCO P-2000 polarimeter with $[\alpha]_{\mathrm{D}}$ values reported in degrees; concentration $(c)$ is in $10 \mathrm{mg} / \mathrm{mL}$. The enantiomeric excesses were determined by chiral HPLC using a Shimadzu Prominence LC-20A instrument with Daicel CHIRALCEL OD-H, CHIRALPAK AD-H or AS-H column. The catalyst B4 was prepared according to the reported procedure. ${ }^{1}$

${ }^{1}$ Müller, S.; Webber, M. J.; List, B. J. Am. Chem. Soc. 2011, 133, 18534-18537. 


\section{Synthesis of the Oxetane Substrates}

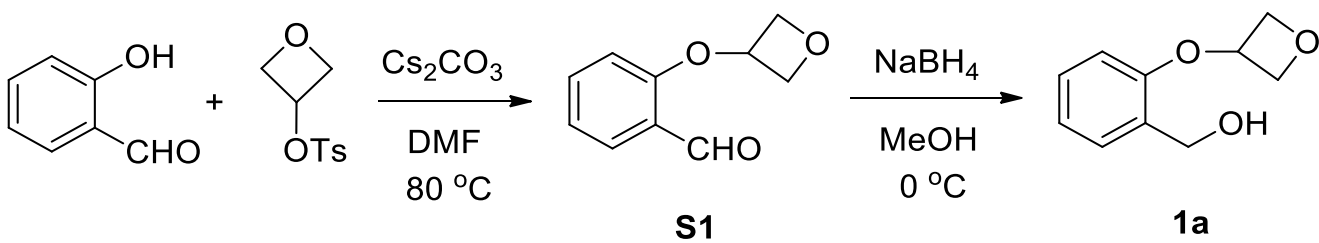

2-(Oxetan-3-yloxy)benzaldehyde (S1). A mixture of salicylaldehyde (2.75 g, 22.5 mmol), oxetan-3-yl 4-methylbenzenesulfonate (3.42 g, $15.0 \mathrm{mmol})$, cesium carbonate $(19.5 \mathrm{~g}, 60.0 \mathrm{mmol})$ in DMF $(30 \mathrm{~mL})$ was stirred overnight at $80{ }^{\circ} \mathrm{C}$ in an oil bath. The mixture was quenched by water $(20 \mathrm{~mL})$, extracted with ethyl ether $(6 \times 50 \mathrm{~mL})$. The organic layers were combined, washed with water $(2 \times 30 \mathrm{~mL})$, dried over anhydrous $\mathrm{Na}_{2} \mathrm{SO}_{4}$, and concentrated under reduced pressure. The residue was purified by silica gel column chromatography (petroleum ether/ethyl acetate $=10: 1$ to $2: 1)$ to afford the desired product $\mathbf{S} 1$ as colorless oil $(2.28 \mathrm{~g}, 85 \%$ yield).

${ }^{1}$ H NMR (400 MHz, $\left.\mathrm{CDCl}_{3}\right) \delta 10.54(\mathrm{~s}, 1 \mathrm{H}), 7.88(\mathrm{~d}, J=7.6 \mathrm{~Hz}, 1 \mathrm{H}), 7.51(\mathrm{t}, J=7.2 \mathrm{~Hz}$, 1H), $7.08(\mathrm{t}, J=7.6 \mathrm{~Hz}, 1 \mathrm{H}), 6.50(\mathrm{~d}, J=8.4 \mathrm{~Hz}, 1 \mathrm{H}), 5.34-5.30(\mathrm{~m}, 1 \mathrm{H}), 5.03(\mathrm{t}, J=6.8$ $\mathrm{Hz}, 2 \mathrm{H}), 4.84(\mathrm{~d}, J=6.4 \mathrm{~Hz}, 2 \mathrm{H}) \mathrm{ppm}$.

${ }^{13} \mathrm{C}$ NMR $\left(100 \mathrm{MHz}, \mathrm{CDCl}_{3}\right) \delta 189.2,158.7,135.9,129.0,124.9,121.6,112.2,77.6,70.7$ ppm.

HRMS (CI) Calcd for $\mathrm{C}_{10} \mathrm{H}_{10} \mathrm{O}_{3}[\mathrm{M}]^{+}:$178.0630, Found: 178.0632.

(2-(Oxetan-3-yloxy)phenyl)methanol (1a). To a solution of 2-(oxetan-3-yloxy)benzaldehyde S1 (446 mg, $2.5 \mathrm{mmol})$ in $\mathrm{MeOH}(5 \mathrm{~mL})$ at $0{ }^{\circ} \mathrm{C}$ was added $\mathrm{NaBH}_{4}$ 
(189 $\mathrm{mg}, 5.0 \mathrm{mmol})$ in portions. After stirring at $0{ }^{\circ} \mathrm{C}$ for $1 \mathrm{~h}$ upon completion, the mixture was slowly quenched by saturated $\mathrm{NH}_{4} \mathrm{Cl}$ aqueous solution $(10 \mathrm{~mL})$, and extracted with dichloromethane $(2 \times 30 \mathrm{~mL})$. The organic layers were combined, dried over anhydrous $\mathrm{Na}_{2} \mathrm{SO}_{4}$, and concentrated under reduced pressure. The residue was purified by silica gel column chromatography (petroleum ether/ethyl acetate $=10: 1$ to $1: 1)$ to afford the desired product 1 a as a white solid (420 mg, $93 \%$ yield).

${ }^{1} \mathrm{H}$ NMR $\left(400 \mathrm{MHz} \mathrm{CDCl}_{3}\right) \delta 7.36(\mathrm{~d}, J=6.8 \mathrm{~Hz}, 1 \mathrm{H}), 7.22(\mathrm{t}, J=7.6 \mathrm{~Hz}, 1 \mathrm{H}), 6.99(\mathrm{t}$, $J=7.6 \mathrm{~Hz}, 1 \mathrm{H}), 6.39(\mathrm{~d}, J=8.4 \mathrm{~Hz}, 1 \mathrm{H}), 5.28-5.22(\mathrm{~m}, 1 \mathrm{H}), 5.00(\mathrm{t}, J=6.8 \mathrm{~Hz}, 2 \mathrm{H})$, $4.77(\mathrm{t}, J=7.2 \mathrm{~Hz}, 2 \mathrm{H}), 4.74(\mathrm{~s}, 2 \mathrm{H}), 2.18$ (br s, 1H) ppm.

${ }^{13} \mathrm{C}$ NMR $\left(100 \mathrm{MHz}, \mathrm{CDCl}_{3}\right) \delta 154.2,129.5,129.2,128.9,121.6,110.9,78.0,70.2,61.5$ ppm.

HRMS (CI) Calcd for $\mathrm{C}_{10} \mathrm{H}_{11} \mathrm{O}_{3}[\mathrm{M}-\mathrm{H}]^{+}:$179.0708, Found: 179.0714 .<smiles>CC(=O)C1=CC#[Se]C=C1O</smiles><smiles>[Os+3]C1COC1</smiles><smiles>CC(C)(C)O[Mg]</smiles><smiles></smiles>

S2-S8<smiles></smiles>

1b-e, 1i-k<smiles>CC(=O)c1ccccc1OC1COC1</smiles>

S2 
1-(2-(Oxetan-3-yloxy)phenyl)ethanone (S2). ${ }^{2}$ A mixture of 1-(2-hydroxy-phenyl)ethanone (2.04 g, $15.0 \mathrm{mmol})$, oxetan-3-yl 4-methylbenzenesulfonate (2.28 g, 10.0 $\mathrm{mmol})$, cesium carbonate $(9.77 \mathrm{~g}, 30.0 \mathrm{mmol})$, and DMF $(20 \mathrm{~mL})$ was stirred at 80 ${ }^{\circ} \mathrm{C}$ in an oil bath for $16 \mathrm{~h}$. The mixture was quenched by water $(20 \mathrm{~mL})$ and extracted with ethyl ether $(6 \times 50 \mathrm{~mL})$. The organic layers were combined, washed with water $(2 \times 30 \mathrm{~mL})$, dried over anhydrous $\mathrm{Na}_{2} \mathrm{SO}_{4}$, and concentrated under reduced pressure. The residue was first purified by silica gel column chromatography (petroleum ether/ethyl acetate $=10: 1$ to $2: 1$ ) to give the desired product $\mathbf{S 2}$ as a white solid (1.56 g, 81\% yield).

${ }^{1} \mathbf{H}$ NMR $\left(400 \mathrm{MHz}, \mathrm{CDCl}_{3}\right) \delta 7.77(\mathrm{~d}, J=7.6 \mathrm{~Hz}, 1 \mathrm{H}), 7.40(\mathrm{t}, J=8.0 \mathrm{~Hz}, 1 \mathrm{H}), 7.04$ $\left(\mathrm{dt}, J_{1}=7.6 \mathrm{~Hz}, J_{2}=1.6 \mathrm{~Hz}, 1 \mathrm{H}\right), 6.45(\mathrm{~d}, J=8.0 \mathrm{~Hz}, 1 \mathrm{H}), 5.32-5.28(\mathrm{~m}, 1 \mathrm{H}), 5.03(\mathrm{t}, J$ $=6.4 \mathrm{~Hz}, 2 \mathrm{H}), 4.82-4.79(\mathrm{~m}, 2 \mathrm{H}), 2.68(\mathrm{~s}, 3 \mathrm{H}) \mathrm{ppm}$.

${ }^{13} \mathrm{C}$ NMR $\left(100 \mathrm{MHz}, \mathrm{CDCl}_{3}\right) \delta 199.2,155.6,133.6,130.9,128.6,121.5,112.1,77.7,70.6$, 31.9 ppm.

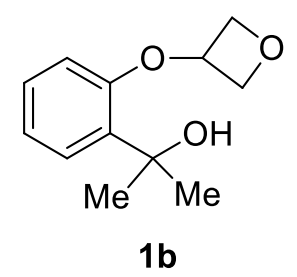

2-(2-(Oxetan-3-yloxy)phenyl)propan-2-ol (1b). ${ }^{2}$ At $0{ }^{\circ} \mathrm{C}$, to a solution of 1-(2(oxetan-3-yloxy)phenyl)ethanone S2 $(384 \mathrm{mg}, 2.0 \mathrm{mmol})$ in THF $(10 \mathrm{~mL})$ was added dropwise $\mathrm{MeMgBr}\left(3.0 \mathrm{mmol}, 1.0 \mathrm{~mL}, 3 \mathrm{M}\right.$ in THF). After stirring at $0^{\circ} \mathrm{C}$ for $1 \mathrm{~h}$, the mixture was slowly quenched with water $(5 \mathrm{~mL})$ and extracted with ethyl acetate $(3 \times 20 \mathrm{~mL})$. The combined organic layers were dried over anhydrous $\mathrm{Na}_{2} \mathrm{SO}_{4}$ and concentrated under reduced pressure. The residue was purified by silica gel column chromatography (petroleum ether/ethyl acetate $=2: 1$ ) to afford the desired product $\mathbf{1 b}$ as a white solid (362 $\mathrm{mg}$, 87\% yield).

2 Yang, W.; Sun, J. Angew. Chem., Int. Ed. 2016, 55, 1868. 
${ }^{1} \mathbf{H}$ NMR $\left(400 \mathrm{MHz}, \mathrm{CDCl}_{3}\right) \delta 7.41\left(\mathrm{dd}, J_{1}=7.6 \mathrm{~Hz}, J_{2}=1.6 \mathrm{~Hz}, 1 \mathrm{H}\right), 7.18\left(\mathrm{dt}, J_{1}=7.6\right.$ $\left.\mathrm{Hz}, J_{2}=1.6 \mathrm{~Hz}, 1 \mathrm{H}\right), 6.99(\mathrm{t}, J=7.6 \mathrm{~Hz}, 1 \mathrm{H}), 6.40(\mathrm{~d}, J=8.0 \mathrm{~Hz}, 1 \mathrm{H}), 5.33-5.28(\mathrm{~m}$, $1 \mathrm{H}), 5.02(\mathrm{t}, J=6.4 \mathrm{~Hz}, 2 \mathrm{H}), 4.80(\mathrm{t}, J=6.4 \mathrm{~Hz}, 2 \mathrm{H}), 3.71(\mathrm{br} \mathrm{s}, 1 \mathrm{H}), 1.66(\mathrm{~s}, 6 \mathrm{H}) \mathrm{ppm}$. ${ }^{13} \mathrm{C}$ NMR $\left(100 \mathrm{MHz}, \mathrm{CDCl}_{3}\right) \delta 153.7,136.2,128.2,126.4,121.8,111.7,77.9,72.5,70.3$, 29.7 ppm.<smiles>CC(=O)c1cc(F)ccc1OC1COC1</smiles>

S3

1-(5-Fluoro-2-(oxetan-3-yloxy)phenyl)ethanone (S3) was prepared from 1-(5fluoro-2-hydroxyphenyl)ethanone (740 $\mathrm{mg}, 4.8 \mathrm{mmol}$ ) and oxetan-3-yl 4methylbenzenesulfonate $(913 \mathrm{mg}, 4.0 \mathrm{mmol}$ ) according to the same procedure for the synthesis of S2 (purification by flash column chromatography: petroleum ether/ethyl acetate $=10: 1$ to 3:1). White solid, $735 \mathrm{mg}$, $87 \%$ yield .

${ }^{1} \mathbf{H}$ NMR $\left(400 \mathrm{MHz} \mathrm{CDCl}_{3}\right) \delta 7.46\left(\mathrm{dd}, J_{1}=8.8 \mathrm{~Hz}, J_{2}=3.2 \mathrm{~Hz}, 1 \mathrm{H}\right), 7.10-7.06(\mathrm{~m}$, $1 \mathrm{H}), 6.41\left(\mathrm{dd}, J_{1}=8.8 \mathrm{~Hz}, J_{2}=4.0 \mathrm{~Hz}, 1 \mathrm{H}\right), 5.28-5.22(\mathrm{~m}, 1 \mathrm{H}), 4.99(\mathrm{t}, J=6.8 \mathrm{~Hz}, 2 \mathrm{H})$, $4.77(\mathrm{t}, J=1.6 \mathrm{~Hz}, 2 \mathrm{H}), 2.65(\mathrm{~s}, 3 \mathrm{H}) \mathrm{ppm}$.

${ }^{13} \mathrm{C}$ NMR (100 MHz, $\left.\mathrm{CDCl}_{3}\right) \delta$ 197.6, $157.2(\mathrm{~d}, J=240.3 \mathrm{~Hz}), 152.0$ (d, J = 2.1 Hz), $129.6(\mathrm{~d}, J=5.8 \mathrm{~Hz}), 120.0(\mathrm{~d}, J=23.4 \mathrm{~Hz}), 117.2(\mathrm{~d}, J=24.0 \mathrm{~Hz}), 113.5(\mathrm{~d}, J=7.5 \mathrm{~Hz})$, 77.6, 71.2, $31.8 \mathrm{ppm}$.

HRMS (CI) Calcd for $\mathrm{C}_{11} \mathrm{H}_{10} \mathrm{FO}_{3}[\mathrm{M}-\mathrm{H}]^{+}:$209.0614, Found: 209.0610.<smiles>CC(C)(O)c1cc(F)ccc1OC1COC1</smiles>

$1 \mathrm{c}$

2-(5-Fluoro-2-(oxetan-3-yloxy)phenyl)propan-2-ol (1c) was prepared from S3 (735 $\mathrm{mg}$, $3.5 \mathrm{mmol})$ and $\mathrm{MeMgBr}(5.25 \mathrm{mmol}, 1.75 \mathrm{~mL}, 3 \mathrm{M}$ in THF) according to the 
same procedure for the synthesis of $\mathbf{1 b}$ (purification by flash column chromatography: petroleum ether/ethyl acetate $=10: 1$ to $\mathrm{CH}_{2} \mathrm{Cl}_{2}$ ). White solid, 642 $\mathrm{mg}, 81 \%$ yield.

${ }^{1}$ H NMR $\left(400 \mathrm{MHz}, \mathrm{CDCl}_{3}\right) \delta 7.18\left(\mathrm{dd}, J_{1}=10.0 \mathrm{~Hz}, J_{2}=2.8 \mathrm{~Hz}, 1 \mathrm{H}\right), 6.87-6.82(\mathrm{~m}$, $1 \mathrm{H}), 6.32\left(\mathrm{dd}, J_{1}=8.8 \mathrm{~Hz}, J_{2}=4.4 \mathrm{~Hz}, 1 \mathrm{H}\right), 5.27-5.22(\mathrm{~m}, 1 \mathrm{H}), 5.00(\mathrm{t}, J=6.8 \mathrm{~Hz}, 2 \mathrm{H})$, $4.77(\mathrm{t}, J=5.6 \mathrm{~Hz}, 2 \mathrm{H}), 3.33$ (br s, 1H), $1.63(\mathrm{~s}, 6 \mathrm{H}) \mathrm{ppm}$.

${ }^{13} \mathrm{C}$ NMR (100 MHz, $\left.\mathrm{CDCl}_{3}\right) \delta 157.6(\mathrm{~d}, J=238.4 \mathrm{~Hz}), 149.7$ (d, J = 2.3 Hz), 138.5 (d, $J=6.0 \mathrm{~Hz}), 114.0(\mathrm{~d}, J=29.4 \mathrm{~Hz}), 113.7(\mathrm{~d}, J=27.7 \mathrm{~Hz}), 112.5(\mathrm{~d}, J=8.2 \mathrm{~Hz}), 77.8$, 72.2, 70.7, 29.5 ppm.

HRMS (CI) Calcd for $\mathrm{C}_{12} \mathrm{H}_{14} \mathrm{FO}_{3}[\mathrm{M}-\mathrm{H}]^{+}$: 225.0927, Found: 225.0932.<smiles>CC(=O)c1cc(Cl)ccc1OC1COC1</smiles>

S4

1-(5-Chloro-2-(oxetan-3-yloxy)phenyl)ethan-1-one (S4) was prepared from 1-(5chloro-2-hydroxyphenyl)ethanone (819 $\mathrm{mg}, 4.8 \mathrm{mmol})$ and oxetan-3-yl 4methylbenzenesulfonate $(913 \mathrm{mg}, 4.0 \mathrm{mmol})$ according to the same procedure for the synthesis of $\mathbf{S 2}$ (purification by flash column chromatography: petroleum ether/ethyl acetate $=10: 1$ to 3:1). White solid, $791 \mathrm{mg}, 87 \%$ yield .

${ }^{1} \mathrm{H}$ NMR $\left(400 \mathrm{MHz}, \mathrm{CDCl}_{3}\right) \delta 7.72(\mathrm{~s}, 1 \mathrm{H}), 7.35-7.33(\mathrm{~m}, 1 \mathrm{H}), 6.40(\mathrm{~d}, J=8.8 \mathrm{~Hz}, 1 \mathrm{H})$, 5.30-5.25 (m, 1H), $5.01(\mathrm{t}, J=6.8 \mathrm{~Hz}, 2 \mathrm{H}), 4.78(\mathrm{t}, J=6.0 \mathrm{~Hz}, 2 \mathrm{H}), 2.66(\mathrm{~s}, 3 \mathrm{H}) \mathrm{ppm}$. ${ }^{13} \mathrm{C}$ NMR $\left(100 \mathrm{MHz}, \mathrm{CDCl}_{3}\right) \delta 197.6,154.2,133.1,130.7,129.7,127.1,113.6,77.5,71.1$, $31.8 \mathrm{ppm}$.

HRMS (CI) Calcd for $\mathrm{C}_{11} \mathrm{H}_{10} \mathrm{ClO}_{3}[\mathrm{M}-\mathrm{H}]^{+}$: 225.0318, Found: 225.0320.<smiles>CC(C)(O)c1cc(Cl)ccc1OC1COC1</smiles> 
2-(5-Chloro-2-(oxetan-3-yloxy)phenyl)propan-2-ol (1d) was prepared from S4 (725 mg, $3.2 \mathrm{mmol})$ and $\mathrm{MeMgBr}(4.8 \mathrm{mmol}, 1.6 \mathrm{~mL}, 3 \mathrm{M}$ in THF) according to the same procedure for the synthesis of $\mathbf{1 b}$ (purification by flash column chromatography: petroleum ether/ethyl acetate $=5: 1$ to $2: 1)$. White solid, $685 \mathrm{mg}$, $88 \%$ yield.

${ }^{1} \mathrm{H}$ NMR $\left(400 \mathrm{MHz}, \mathrm{CDCl}_{3}\right) \delta 7.43(\mathrm{~s}, 1 \mathrm{H}), 7.12(\mathrm{~d}, J=8.8 \mathrm{~Hz}, 1 \mathrm{H}), 6.31(\mathrm{~d}, J=8.4 \mathrm{~Hz}$, $1 \mathrm{H}), 5.28-5.23(\mathrm{~m}, 1 \mathrm{H}), 5.0(\mathrm{t}, J=6.8 \mathrm{~Hz}, 2 \mathrm{H}), 4.76(\mathrm{t}, J=5.6 \mathrm{~Hz}, 2 \mathrm{H}), 3.21(\mathrm{br} \mathrm{s}, 1 \mathrm{H})$, $1.64(\mathrm{~s}, 6 \mathrm{H}) \mathrm{ppm}$.

${ }^{13} \mathrm{C}$ NMR $\left(100 \mathrm{MHz}, \mathrm{CDCl}_{3}\right) \delta$ 152.2, 138.3, 127.7, 127.0, 126.9, 112.9, 77.7, 72.3, 70.6, 29.5 ppm.

HRMS (CI) Calcd for $\mathrm{C}_{12} \mathrm{H}_{14} \mathrm{ClO}_{3}[\mathrm{M}-\mathrm{H}]^{+}:$241.0631, Found: 241.0636.<smiles>CC(=O)c1cc(Br)ccc1OC1COC1</smiles>

S5

1-(5-Bromo-2-(oxetan-3-yloxy)phenyl)ethan-1-one (S5) was prepared from 1-(5bromo-2-hydroxyphenyl)ethanone (1.03 g, $4.8 \mathrm{mmol}$ ) and oxetan-3-yl 4-methylbenzenesulfonate $(913 \mathrm{mg}, 4.0 \mathrm{mmol})$ according to the same procedure for the synthesis of S2 (purification by flash column chromatography: petroleum ether/ethyl acetate $=10: 1$ to 3:1). White solid, $672 \mathrm{mg}, 62 \%$ yield .

${ }^{1} \mathbf{H}$ NMR $\left(400 \mathrm{MHz}, \mathrm{CDCl}_{3}\right) \delta 7.76(\mathrm{~d}, J=1.6 \mathrm{~Hz}, 1 \mathrm{H}), 7.48\left(\mathrm{dd}, J_{1}=9.6 \mathrm{~Hz}, J_{2}=6.0\right.$ $\mathrm{Hz}, 1 \mathrm{H}), 6.34(\mathrm{~d}, J=7.6 \mathrm{~Hz}, 1 \mathrm{H}), 5.30-5.27(\mathrm{~m}, 1 \mathrm{H}), 5.01(\mathrm{t}, J=6.4 \mathrm{~Hz}, 2 \mathrm{H}), 4.78(\mathrm{t}, J$ $=4.2 \mathrm{~Hz}, 2 \mathrm{H}), 2.65(\mathrm{~s}, 3 \mathrm{H}) \mathrm{ppm}$.

${ }^{13} \mathrm{C}$ NMR $\left(100 \mathrm{MHz}, \mathrm{CDCl}_{3}\right) \delta 197.6,154.7,136.1,133.6,130.1,114.2,114.0,77.5,71.0$, $31.8 \mathrm{ppm}$.

HRMS (CI) Calcd for $\mathrm{C}_{11} \mathrm{H}_{10} \mathrm{BrO}_{3}[\mathrm{M}-\mathrm{H}]^{+}:$268.9813, Found: 268.9810. 
<smiles>CC(C)(O)c1cc(Br)ccc1OC1COC1</smiles>

2-(5-Bromo-2-(oxetan-3-yloxy)phenyl)propan-2-ol (1e) was prepared from S5 (651 $\mathrm{mg}$, $2.4 \mathrm{mmol})$ and $\mathrm{MeMgBr}(3.6 \mathrm{mmol}, 1.2 \mathrm{~mL}, 3 \mathrm{M}$ in THF) according to the same procedure for the synthesis of $\mathbf{1 b}$ (purification by flash column chromatography: petroleum ether/ethyl acetate $=5: 1$ to $2: 1$ ). White solid, $527 \mathrm{mg}$, $76 \%$ yield .

${ }^{1} \mathrm{H}$ NMR $\left(400 \mathrm{MHz}, \mathrm{CDCl}_{3}\right) \delta 7.59(\mathrm{~s}, 1 \mathrm{H}), 7.27(\mathrm{~d}, J=6.8 \mathrm{~Hz}, 1 \mathrm{H}), 6.26(\mathrm{~d}, J=8.4 \mathrm{~Hz}$, 1H), 5.28-5.22 (m, 1H), $5.00(\mathrm{t}, J=6.8 \mathrm{~Hz}, 2 \mathrm{H}), 4.76(\mathrm{t}, J=6.0 \mathrm{~Hz}, 2 \mathrm{H}), 3.41(\mathrm{br} \mathrm{s}, 1 \mathrm{H})$, $1.64(\mathrm{~s}, 6 \mathrm{H}) \mathrm{ppm}$.

${ }^{13} \mathrm{C}$ NMR $\left(100 \mathrm{MHz}, \mathrm{CDCl}_{3}\right) \delta 152.6,138.7,130.7,129.7,114.4,113.4,77.7,72.2,70.5$, 29.5 ppm.

HRMS (CI) Calcd for $\mathrm{C}_{12} \mathrm{H}_{14} \mathrm{BrO}_{3}[\mathrm{M}-\mathrm{H}]^{+}$: 285.0126, Found: 285.0132.<smiles>CC(=O)c1cc(OCc2ccccc2)ccc1OC1COC1</smiles>

S6

1-(5-(Benzyloxy)-2-(oxetan-3-yloxy)phenyl)ethan-1-one (S6) was prepared from 1-(5-benzyloxy-2-hydroxyphenyl)ethanone (2.66 g, $11.0 \mathrm{mmol})$ and oxetan-3-yl 4methylbenzenesulfonate $(1.66 \mathrm{~g}, 7.3 \mathrm{mmol})$ according to the same procedure for the synthesis of $\mathbf{S 2}$ (purification by flash column chromatography: petroleum ether/ethyl acetate $=5: 1$ to1:1). White solid, $2.17 \mathrm{~g}$, 99\% yield.

${ }^{1} \mathbf{H}$ NMR $\left(400 \mathrm{MHz}, \mathrm{CDCl}_{3}\right) \delta 7.43-7.30(\mathrm{~m}, 6 \mathrm{H}), 7.03\left(\mathrm{dd}, J_{1}=9.2 \mathrm{~Hz}, J_{2}=3.2 \mathrm{~Hz}\right.$, $1 \mathrm{H}), 6.40(\mathrm{~d}, J=8.8 \mathrm{~Hz}, 1 \mathrm{H}), 5.26-5.21(\mathrm{~m}, 1 \mathrm{H}), 5.03(\mathrm{~s}, 2 \mathrm{H}), 4.99(\mathrm{t}, J=3.2 \mathrm{~Hz}, 2 \mathrm{H})$, $4.79(\mathrm{t}, J=5.6 \mathrm{~Hz}, 2 \mathrm{H}), 2.68(\mathrm{~s}, 3 \mathrm{H}) \mathrm{ppm}$.

${ }^{13} \mathrm{C}$ NMR $\left(100 \mathrm{MHz}, \mathrm{CDCl}_{3}\right) \delta 198.8,153.2,150.3,136.7,128.8,128.6,128.1,127.6$, 121.0, 115.7, 113.6, 77.9, 71.0, 70.6, $32.0 \mathrm{ppm}$.

HRMS (CI) Calcd for $\mathrm{C}_{18} \mathrm{H}_{17} \mathrm{O}_{4}[\mathrm{M}-\mathrm{H}]^{+}$: 297.1127, Found: 297.1132. 
<smiles>CC(C)(O)c1cc(OCc2ccccc2)ccc1OC1COC1</smiles>

2-(5-(Benzyloxy)-2-(oxetan-3-yloxy)phenyl)propan-2-ol (1i) was prepared from S6 (596 mg, $2.0 \mathrm{mmol})$ and $\mathrm{MeMgBr}(6.0 \mathrm{mmol}, 2.0 \mathrm{~mL}, 3 \mathrm{M}$ in THF) according to the same procedure for the synthesis of $\mathbf{1 b}$ (purification by flash column chromatography: petroleum ether/ethyl acetate $=5: 1$ to $3: 1)$. White solid, $550 \mathrm{mg}$, $87 \%$ yield.

${ }^{1}$ H NMR (400 MHz, $\left.\mathrm{CDCl}_{3}\right) \delta$ 7.43-7.33 (m, 5H), $7.10(\mathrm{~d}, J=2.4 \mathrm{~Hz}, 1 \mathrm{H}), 6.74\left(\mathrm{dd}, J_{1}\right.$ $\left.=8.8 \mathrm{~Hz}, J_{2}=5.2 \mathrm{~Hz}, 1 \mathrm{H}\right), 6.30(\mathrm{~d}, J=8.8 \mathrm{~Hz}, 1 \mathrm{H}), 5.26-5.20(\mathrm{~m}, 1 \mathrm{H}), 5.00(\mathrm{t}, J=2.8$ $\mathrm{Hz}, 4 \mathrm{H}), 4.78(\mathrm{t}, J=6.0 \mathrm{~Hz}, 2 \mathrm{H}), 3.26$ (br s, 1H), $1.64(\mathrm{~s}, 6 \mathrm{H}) \mathrm{ppm}$.

${ }^{13} \mathrm{C}$ NMR $\left(100 \mathrm{MHz}_{2} \mathrm{CDCl}_{3}\right) \delta 153.4,147.9,137.6,137.0,128.6,128.0,127.6,114.7$, $112.4,112.3,78.0,72.5,70.6,70.5,29.6 \mathrm{ppm}$.

HRMS (CI) Calcd for $\mathrm{C}_{19} \mathrm{H}_{21} \mathrm{O}_{4}[\mathrm{M}-\mathrm{H}]^{+}:$313.1440, Found: 313.1455.<smiles>C=CCOc1ccc(OC2COC2)c(C(C)=O)c1</smiles>

S7

1-(5-(Allyloxy)-2-(oxetan-3-yloxy)phenyl)ethan-1-one (S7) was prepared from 1(5-allyloxy-2-hydroxyphenyl)ethanone (1.44 g, $7.5 \mathrm{mmol})$ and oxetan-3-yl 4methylbenzenesulfonate $(1.14 \mathrm{~g}, 5.0 \mathrm{mmol})$ according to the same procedure for the synthesis of $\mathbf{S 2}$ (purification by flash column chromatography: petroleum ether/ethyl acetate $=5: 1$ to 1:1). White solid, $1.21 \mathrm{~g}$, 97\% yield .

${ }^{1} \mathrm{H}$ NMR $\left(400 \mathrm{MHz}, \mathrm{CDCl}_{3}\right) \delta 7.32(\mathrm{~d}, J=3.2 \mathrm{~Hz}, 1 \mathrm{H}), 6.98\left(\mathrm{dd}, J_{1}=7.2 \mathrm{~Hz}, J_{2}=3.2\right.$ $\mathrm{Hz}, 1 \mathrm{H}), 6.39$ (d, J = 8.8 Hz, 1H), 6.06-5.90 (m, 1H), 5.39 (d, J = 17.2 Hz, 1H), 5.28$5.20(\mathrm{~m}, 2 \mathrm{H}), 4.99(\mathrm{t}, J=7.2 \mathrm{~Hz}, 2 \mathrm{H}), 4.78(\mathrm{t}, J=5.6 \mathrm{~Hz}, 2 \mathrm{H}), 4.50(\mathrm{~d}, J=5.2 \mathrm{~Hz}, 2 \mathrm{H})$, $2.66(\mathrm{~s}, 3 \mathrm{H}) \mathrm{ppm}$. 
${ }^{13} \mathrm{C}$ NMR $\left(100 \mathrm{MHz}, \mathrm{CDCl}_{3}\right) \delta 198.7,153.0,150.2,133.0,128.7,121.0,117.9,115.6$, 113.5, 77.8, 71.0, 69.4, 32.0 ppm.

HRMS (CI) Calcd for $\mathrm{C}_{14} \mathrm{H}_{15} \mathrm{O}_{4}[\mathrm{M}-\mathrm{H}]^{+}:$247.0970, Found: 247.0981.<smiles>C=CCOc1ccc(OC2COC2)c(C(C)(C)O)c1</smiles>

$1 \mathrm{j}$

2-(5-(Allyloxy)-2-(oxetan-3-yloxy)phenyl)propan-2-ol (1j) was prepared from S7 (744 mg, $3.0 \mathrm{mmol})$ and $\mathrm{MeMgBr}(9.0 \mathrm{mmol}, 3.0 \mathrm{~mL}, 3 \mathrm{M}$ in THF) according to the same procedure for the synthesis of $\mathbf{1 b}$ (purification by flash column chromatography: petroleum ether/ethyl acetate $=5: 1$ to $3: 1$ ). Pale yellow oil, 460 $\mathrm{mg}, 58 \%$ yield.

${ }^{1}$ H NMR (400 MHz, $\left.\mathrm{CDCl}_{3}\right) \delta 7.03(\mathrm{~s}, 1 \mathrm{H}), 6.67(\mathrm{~d}, J=8.8 \mathrm{~Hz}, 1 \mathrm{H}), 6.29(\mathrm{~d}, J=8.8 \mathrm{~Hz}$, 1H), 6.07-5.98 (m, 1H), $5.39(\mathrm{~d}, J=13.2 \mathrm{~Hz}, 1 \mathrm{H}), 5.26-5.20(\mathrm{~m}, 2 \mathrm{H}), 4.99(\mathrm{t}, J=6.8 \mathrm{~Hz}$, $2 \mathrm{H}), 4.77(\mathrm{t}, J=6.0 \mathrm{~Hz}, 2 \mathrm{H}), 4.48(\mathrm{~d}, J=5.3 \mathrm{~Hz}, 2 \mathrm{H}), 3.46$ (br s, $1 \mathrm{H}), 1.62(\mathrm{~s}, 6 \mathrm{H}) \mathrm{ppm}$. ${ }^{13} \mathrm{C}$ NMR $\left(100 \mathrm{MHz}, \mathrm{CDCl}_{3}\right) \delta 153.2,147.9,137.4,133.3,117.7,114.5,112.2,112.3$, 78.0, 72.4, 70.5, 69.4, $29.6 \mathrm{ppm}$.

HRMS (CI) Calcd for $\mathrm{C}_{15} \mathrm{H}_{19} \mathrm{O}_{4}[\mathrm{M}-\mathrm{H}]^{+}:$263.1283, Found: 263.1888.<smiles>[Y5]C(=O)c1cc(OCC#C)ccc1OC1COC1</smiles>

1-(2-(Oxetan-3-yloxy)-5-(prop-2-yn-1-yloxy)phenyl)ethan-1-one (S8) was prepared from 1-(2-hydroxy-5-(prop-2-yn-1-yloxy)phenyl)ethanone (1.42 g, 7.5 $\mathrm{mmol})$ and oxetan-3-yl 4-methylbenzenesulfonate (1.14 g, $5.0 \mathrm{mmol})$ according to the same procedure for the synthesis of $\mathbf{S 2}$ (purification by flash column chromatography: petroleum ether/ethyl acetate $=5: 1$ to $1: 1)$. White solid, $1.04 \mathrm{~g}, 85 \%$ yield. 
${ }^{1} \mathbf{H}$ NMR $\left(400 \mathrm{MHz}, \mathrm{CDCl}_{3}\right) \delta 7.39(\mathrm{~d}, J=2.8 \mathrm{~Hz}, 1 \mathrm{H}), 7.04\left(\mathrm{dd}, J_{1}=9.2 \mathrm{~Hz}, J_{2}=3.2\right.$ $\mathrm{Hz}, 1 \mathrm{H}), 6.41(\mathrm{~d}, J=8.8 \mathrm{~Hz}, 1 \mathrm{H}), 5.28-5.22(\mathrm{~m}, 1 \mathrm{H}), 4.99(\mathrm{t}, J=2.8 \mathrm{~Hz}, 2 \mathrm{H}), 4.77(\mathrm{t}, J$ $=6.0 \mathrm{~Hz}, 2 \mathrm{H}), 4.65(\mathrm{~d}, J=1.2 \mathrm{~Hz}, 2 \mathrm{H}), 2.66(\mathrm{~s}, 3 \mathrm{H}), 2.51(\mathrm{~s}, 1 \mathrm{H}) \mathrm{ppm}$.

${ }^{13} \mathrm{C}$ NMR $\left(100 \mathrm{MHz}, \mathrm{CDCl}_{3}\right) \delta 198.5,151.9,150.7,128.8,121.1,116.1,113.5,78.3,77.8$, 75.8, 71.0, 56.5, 32.0 ppm.

HRMS (CI) Calcd for $\mathrm{C}_{14} \mathrm{H}_{13} \mathrm{O}_{4}[\mathrm{M}-\mathrm{H}]^{+}:$245.0814, Found: 245.0829.<smiles>C#CCOc1ccc(OC2COC2)c(C(C)(C)O)c1</smiles>

2-(2-(Oxetan-3-yloxy)-5-(prop-2-yn-1-yloxy)phenyl)propan-2-ol (1k) was prepared from S8 $(660 \mathrm{mg}, 2.68 \mathrm{mmol})$ and $\mathrm{MeMgBr}(8.04 \mathrm{mmol}, 2.68 \mathrm{~mL}, 3 \mathrm{M}$ in $\mathrm{THF}$ ) according to the same procedure for the synthesis of $\mathbf{1 b}$ (purification by flash column chromatography: petroleum ether/ethyl acetate $=5: 1$ to 3:1). Pale yellow oil, $322 \mathrm{mg}$, 49\% yield.

${ }^{1} \mathbf{H}$ NMR $\left(400 \mathrm{MHz}, \mathrm{CDCl}_{3}\right) \delta 7.09(\mathrm{~d}, J=2.8 \mathrm{~Hz}, 1 \mathrm{H}), 6.77\left(\mathrm{dd}, J_{1}=8.8 \mathrm{~Hz}, J_{2}=2.8\right.$ $\mathrm{Hz}, 1 \mathrm{H}), 6.30(\mathrm{~d}, J=8.8 \mathrm{~Hz}, 1 \mathrm{H}), 5.24-5.18(\mathrm{~m}, 1 \mathrm{H}), 4.97(\mathrm{t}, J=6.8 \mathrm{~Hz}, 2 \mathrm{H}), 4.75(\mathrm{t}, J$ $=5.6 \mathrm{~Hz}, 2 \mathrm{H}), 4.61(\mathrm{~s}, 2 \mathrm{H}), 3.40(\mathrm{br} \mathrm{s}, 1 \mathrm{H}), 2.51(\mathrm{~s}, 1 \mathrm{H}), 1.62(\mathrm{~s}, 6 \mathrm{H}) \mathrm{ppm}$.

${ }^{13} \mathrm{C}$ NMR $\left(100 \mathrm{MHz}, \mathrm{CDCl}_{3}\right) \delta 152.1,148.4,137.8,114.8,112.8,112.3,78.7,78.0,75.6$, 72.4, 70.5, 56.4, 29.6 ppm.

HRMS (CI) Calcd for $\mathrm{C}_{15} \mathrm{H}_{17} \mathrm{O}_{4}[\mathrm{M}-\mathrm{H}]^{+}:$261.1127, Found: 261.1130.<smiles>[Y]c1c[R11]ccc1C(=O)O[Na]</smiles>
$X=O, S$

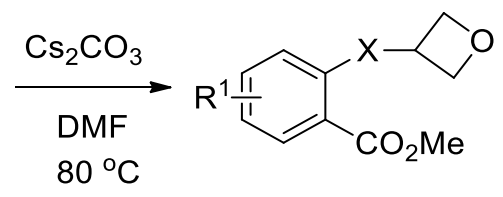

S9-S12

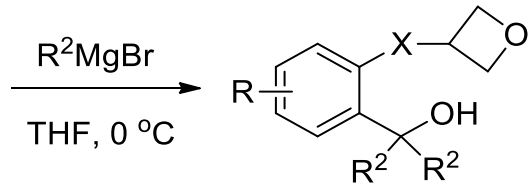

1f, 1g-I, 1n 
<smiles>COc1ccc(Cl)cc1OC1COC1</smiles>

S9

Methyl 4-chloro-2-(oxetan-3-yloxy)benzoate (S9). A mixture of methyl 4-chloro2-hydroxybenzoate (1.40 g, $7.5 \mathrm{mmol})$, oxetan-3-yl 4-methylbenzenesulfonate (1.14 g, $5.0 \mathrm{mmol})$, cesium carbonate $(4.89 \mathrm{~g}, 15.0 \mathrm{mmol})$, and DMF $(10 \mathrm{~mL})$ was stirred at $80^{\circ} \mathrm{C}$ in an oil bath overnight. The mixture was quenched by water $(15 \mathrm{~mL})$ and extracted with ethyl ether $(6 \times 30 \mathrm{~mL})$. The organic layers were combined, washed with water $(2 \times 30 \mathrm{~mL})$, dried over anhydrous $\mathrm{Na}_{2} \mathrm{SO}_{4}$, and concentrated under reduced pressure. The residue was purified by silica gel column chromatography (petroleum ether/ethyl acetate $=10: 1$ to $\mathrm{CH}_{2} \mathrm{Cl}_{2}$ )) to give the desired product $\mathbf{S 9}$ as a white solid (832 $\mathrm{mg}, 69 \%$ yield).

${ }^{1}$ H NMR $\left(400 \mathrm{MHz}, \mathrm{CDCl}_{3}\right) \delta 7.78(\mathrm{~d}, J=8.4 \mathrm{~Hz}, 1 \mathrm{H}), 7.0(\mathrm{~d}, J=8.0 \mathrm{~Hz}, 1 \mathrm{H}), 6.45(\mathrm{~s}$, 1H), 5.25-5.19 (m, 1H), $4.98(\mathrm{t}, J=6.4 \mathrm{~Hz}, 2 \mathrm{H}), 4.80(\mathrm{t}, J=6.4 \mathrm{~Hz}, 2 \mathrm{H}), 3.88(\mathrm{~s}, 3 \mathrm{H})$ ppm.

${ }^{13} \mathrm{C}$ NMR $\left(100 \mathrm{MHz} \mathrm{CDCl}_{3}\right) \delta 165.5,156.8,139.3,133.4,121.5,119.0,113.6,77.6,71.3$, $52.2 \mathrm{ppm}$.

HRMS (CI) Calcd for $\mathrm{C}_{11} \mathrm{H}_{10} \mathrm{ClO}_{4}[\mathrm{M}-\mathrm{H}]^{+}:$241.0268, Found: 241.0262.<smiles>CC(C)(O)c1ccc(Cl)cc1OC1COC1</smiles>

2-(4-Chloro-2-(oxetan-3-yloxy)phenyl)propan-2-ol (1f). At $0{ }^{\circ} \mathrm{C}$, to a solution of methyl 4-chloro-2-(oxetan-3-yloxy)benzoate $\mathbf{S 9}$ (728 mg, $3.0 \mathrm{mmol})$ in THF (15 mL) was added dropwise $\mathrm{MeMgBr}(10.0 \mathrm{mmol}, 3.3 \mathrm{~mL}, 3 \mathrm{M}$ in THF). The mixture was warmed to room temperature and stirred overnight. The reaction was slowly quenched with water $(10 \mathrm{~mL})$ and extracted with ethyl acetate $(3 \times 25 \mathrm{~mL})$. The combined organic layers were dried over anhydrous $\mathrm{Na}_{2} \mathrm{SO}_{4}$ and concentrated 
under reduced pressure. The residue was purified by silica gel column chromatography (petroleum ether/ethyl acetate $=4: 1$ to $2: 1$ ) to afford the desired product 1 f as a white solid (639 $\mathrm{mg}, 89 \%$ yield).

${ }^{1}$ H NMR (400 MHz, $\left.\mathrm{CDCl}_{3}\right) \delta 7.38(\mathrm{~d}, J=8.4 \mathrm{~Hz}, 1 \mathrm{H}), 6.96(\mathrm{~d}, J=8.4 \mathrm{~Hz}, 1 \mathrm{H}), 6.36$ (s, 1H), $5.28-5.25(\mathrm{~m}, 1 \mathrm{H}), 5.03(\mathrm{t}, J=6.8 \mathrm{~Hz}, 2 \mathrm{H}), 4.77(\mathrm{t}, J=6.0 \mathrm{~Hz}, 2 \mathrm{H}), 3.23(\mathrm{br} \mathrm{s}, 1 \mathrm{H})$, $1.63(\mathrm{~s}, 6 \mathrm{H}) \mathrm{ppm}$.

${ }^{13} \mathrm{C}$ NMR (100 MHz, $\left.\mathrm{CDCl}_{3}\right) \delta 154.1,135.1,133.3,127.5,121.7,112.4,77.6,72.2,70.7$, 29.6 ppm.

HRMS (CI) Calcd for $\mathrm{C}_{12} \mathrm{H}_{14} \mathrm{ClO}_{3}[\mathrm{M}-\mathrm{H}]^{+}:$241.0631, Found: 241.0628.<smiles>COC(=O)c1ccc(C)cc1OC1COC1</smiles>

S10

Methyl 4-methyl-2-(oxetan-3-yloxy)benzoate (S10) was prepared from methyl 4methyl-2-hydroxybenzoate (997 mg, $6.0 \mathrm{mmol}$ ) and oxetan-3-yl 4-methylbenzenesulfonate $(913 \mathrm{mg}, 4.0 \mathrm{mmol}$ ) according to the same procedure for the synthesis of S9 (purification by flash column chromatography: petroleum ether/ethyl acetate $=10: 1$ to 3:1). Colorless oil, $707 \mathrm{mg}$, 80\% yield.

${ }^{1} \mathrm{H}$ NMR $\left(400 \mathrm{MHz}, \mathrm{CDCl}_{3}\right) \delta 7.75(\mathrm{~d}, J=8.0 \mathrm{~Hz}, 1 \mathrm{H}), 6.83(\mathrm{~d}, J=8.0 \mathrm{~Hz}, 1 \mathrm{H}), 6.27(\mathrm{~s}$, $1 \mathrm{H}), 5.27-5.23(\mathrm{~m}, 1 \mathrm{H}), 4.97(\mathrm{t}, J=6.8 \mathrm{~Hz}, 2 \mathrm{H}), 4.83(\mathrm{t}, J=6.0 \mathrm{~Hz}, 2 \mathrm{H}), 3.88(\mathrm{~s}, 3 \mathrm{H})$, $2.65(\mathrm{~s}, 3 \mathrm{H}) \mathrm{ppm}$.

${ }^{13} \mathrm{C}$ NMR $\left(100 \mathrm{MHz}, \mathrm{CDCl}_{3}\right) \delta 165.3,155.4,143.6,131.3,121.0,116.6,112.9,77.0,69.9$, 50.9, 20.9 ppm.

HRMS (CI) Calcd for $\mathrm{C}_{12} \mathrm{H}_{13} \mathrm{O}_{4}[\mathrm{M}-\mathrm{H}]^{+}:$221.0814, Found: 221.0820.<smiles>Cc1ccc(C(C)(C)O)c(OC2COC2)c1</smiles>

$1 \mathrm{~g}$ 
2-(4-Methyl-2-(oxetan-3-yloxy)phenyl)propan-2-ol (1g) as prepared from S10 (556 $\mathrm{mg}$, $2.5 \mathrm{mmol})$ and $\mathrm{MeMgBr}(7.5 \mathrm{mmol}, 2.5 \mathrm{~mL}, 3 \mathrm{M}$ in THF) according to the same procedure for the synthesis of $\mathbf{1 f}$ (purification by flash column chromatography: petroleum ether/ethyl acetate $=3: 1$ to $2: 1$ ). White solid, $451 \mathrm{mg}, 81 \%$ yield .

${ }^{1} \mathrm{H}$ NMR $\left(400 \mathrm{MHz} \mathrm{CDCl}_{3}\right) \delta 7.20(\mathrm{~d}, J=8.4 \mathrm{~Hz}, 1 \mathrm{H}), 6.72(\mathrm{~d}, J=7.6 \mathrm{~Hz}, 1 \mathrm{H}), 6.12(\mathrm{~s}$, 1H), 5.24-5.19 (m, 1H), $4.95(\mathrm{t}, J=6.4 \mathrm{~Hz}, 2 \mathrm{H}), 4.72(\mathrm{t}, J=6.0 \mathrm{~Hz}, 2 \mathrm{H}), 3.22(\mathrm{~s}, 3 \mathrm{H})$, $1.56(\mathrm{~s}, 6 \mathrm{H}) \mathrm{ppm}$.

${ }^{13} \mathrm{C}$ NMR $\left(100 \mathrm{MHz}, \mathrm{CDCl}_{3}\right) \delta 153.6,138.1,133.3,126.2,122.2,112.6,78.0,72.3,70.2$, 29.7, 21.2 ppm.

HRMS (CI) Calcd for $\mathrm{C}_{13} \mathrm{H}_{17} \mathrm{O}_{3}[\mathrm{M}-\mathrm{H}]^{+}:$221.1178, Found: 221.1188.<smiles>COc1ccc(OC2COC2)c(C(=O)O)c1</smiles>

Methyl 5-methoxy-2-(oxetan-3-yloxy)benzoate (S11) was prepared from methyl 4-methoxy-2-hydroxybenzoate (1.09 g, $6.0 \mathrm{mmol})$ and oxetan-3-yl 4-methylbenzenesulfonate $(913 \mathrm{mg}, 4.0 \mathrm{mmol})$ according to the same procedure for the synthesis of S9 (purification by flash column chromatography: petroleum ether/ethyl acetate $=5: 1$ to 3:1). White solid, $924 \mathrm{mg}$, 39\% yield.

${ }^{1} \mathbf{H}$ NMR $\left(400 \mathrm{MHz}, \mathrm{CDCl}_{3}\right) \delta 7.34(\mathrm{~d}, J=2.8 \mathrm{~Hz}, 1 \mathrm{H}), 6.95\left(\mathrm{dd}, J_{1}=8.8 \mathrm{~Hz}, J_{2}=3.2\right.$ $\mathrm{Hz}, 1 \mathrm{H}), 6.47(\mathrm{~d}, J=5.2 \mathrm{~Hz}, 1 \mathrm{H}), 5.19-5.13(\mathrm{~m}, 1 \mathrm{H}), 4.91(\mathrm{t}, J=6.4 \mathrm{~Hz}, 2 \mathrm{H}), 4.80(\mathrm{t}, J$ $=5.6 \mathrm{~Hz}, 2 \mathrm{H}), 3.88(\mathrm{~s}, 3 \mathrm{H}), 3.77(\mathrm{~s}, 3 \mathrm{H}) \mathrm{ppm}$.

${ }^{13} \mathrm{C}$ NMR $\left(100 \mathrm{MHz} \mathrm{CDCl}_{3}\right) \delta 166.2,153.8,150.4,121.4,119.5,116.4,115.4,78.1,71.8$, 55.8, 52.1 ppm.

HRMS (CI) Calcd for $\mathrm{C}_{12} \mathrm{H}_{13} \mathrm{O}_{5}[\mathrm{M}-\mathrm{H}]^{+}:$237.0763, Found: 237.0772. 
<smiles>COc1ccc(OC2COC2)c(C(C)(C)O)c1</smiles>

2-(5-Methoxy-2-(oxetan-3-yloxy)phenyl)propan-2-ol (1h) was prepared from S11 ( $1.20 \mathrm{~g}, 5.04 \mathrm{mmol})$ and $\mathrm{MeMgBr}(15.12 \mathrm{mmol}, 5.04 \mathrm{~mL}, 3 \mathrm{M}$ in THF) according to the same procedure for the synthesis of $1 f$ (purification by flash column chromatography: petroleum ether/ethyl acetate $=4: 1$ to $2: 1$ ). White solid, $1.10 \mathrm{~g}, 92 \%$ yield.

${ }^{1} \mathrm{H}$ NMR $\left(400 \mathrm{MHz}, \mathrm{CDCl}_{3}\right) \delta 7.02(\mathrm{~s}, 1 \mathrm{H}), 6.65(\mathrm{~d}, J=8.8 \mathrm{~Hz}, 1 \mathrm{H}), 6.30(\mathrm{~d}, J=8.8 \mathrm{~Hz}$, 1H), 5.23-5.18 (m, 1H), $4.97(\mathrm{t}, J=6.8 \mathrm{~Hz}, 2 \mathrm{H}), 4.75(\mathrm{t}, J=5.6 \mathrm{~Hz}, 2 \mathrm{H}), 3.74(\mathrm{~s}, 3 \mathrm{H})$, 3.49 (br s, 1H), $1.62(\mathrm{~s}, 6 \mathrm{H})$ ppm.

${ }^{13} \mathrm{C}$ NMR $\left(100 \mathrm{MHz}, \mathrm{CDCl}_{3}\right) \delta 154.2,147.4,137.7,113.6,112.4,111.4,78.0,72.4,70.5$, 55.7, 29.6 ppm.

HRMS (CI) Calcd for $\mathrm{C}_{13} \mathrm{H}_{17} \mathrm{O}_{4}[\mathrm{M}-\mathrm{H}]^{+}:$237.1127, Found: 237.1140.

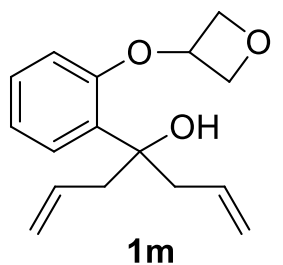

4-(2-(Oxetan-3-yloxy)phenyl)hepta-1,6-dien-4-ol (1m) was prepared from methyl 2-(oxetan-3-yloxy)benzoate (1.35 g, $6.5 \mathrm{mmol})$ and allylmagnesium bromide (19.5 $\mathrm{mmol}, 19.5 \mathrm{~mL}, 1.0 \mathrm{M}$ in THF) according to the same procedure for the synthesis of 1f (purification by flash column chromatography: petroleum ether/ethyl acetate = 10:1 to 5:1). White solid, $764 \mathrm{mg}, 45 \%$ yield.

${ }^{1} \mathbf{H}$ NMR $\left(400 \mathrm{MHz}, \mathrm{CDCl}_{3}\right) \delta 7.47(\mathrm{~d}, J=8.0 \mathrm{~Hz}, 1 \mathrm{H}), 7.17(\mathrm{t}, J=7.6 \mathrm{~Hz}, 1 \mathrm{H}), 6.98(\mathrm{t}$, $J=7.2 \mathrm{~Hz}, 1 \mathrm{H}), 6.35(\mathrm{~d}, J=8.0 \mathrm{~Hz}, 1 \mathrm{H}), 5.66-5.56(\mathrm{~m}, 2 \mathrm{H}), 5.29-5.23(\mathrm{~m}, 1 \mathrm{H}), 5.10(\mathrm{~s}$, 1H), 5.05-5.0 (m, 5H), $4.77(\mathrm{t}, J=6.0 \mathrm{~Hz}, 2 \mathrm{H}), 3.06-3.01(\mathrm{~m}, 2 \mathrm{H}), 2.89$ (br s, $1 \mathrm{H}), 2.61-$ $2.58(\mathrm{~m}, 2 \mathrm{H}) \mathrm{ppm}$. 
${ }^{13} \mathrm{C}$ NMR $\left(100 \mathrm{MHz}, \mathrm{CDCl}_{3}\right) \delta 152.8,134.1,133.2,128.6,128.3,121.5,118.3,111.5$, 78.0, 75.3, 70.2, $44.5 \mathrm{ppm}$.

HRMS (CI) Calcd for $\mathrm{C}_{16} \mathrm{H}_{19} \mathrm{O}_{3}[\mathrm{M}-\mathrm{H}]^{+}:$259.1334, Found: 259.1316.<smiles>CCC(=O)c1ccccc1O</smiles><smiles>[Os+3]C1COC1</smiles><smiles>CCC(=O)c1ccccc1OC1CCC1</smiles>

S12

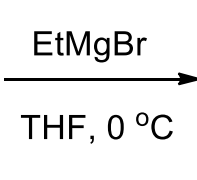

1-(2-(Oxetan-3-yloxy)phenyl)propan-1-one (S12). A mixture of 1-(2-hydroxyphenyl)propan-1-one (1.35 g, $9.0 \mathrm{mmol})$, oxetan-3-yl 4-methylbenzenesulfonate (1.37 g, $6.0 \mathrm{mmol})$, cesium carbonate ( $4.89 \mathrm{~g}, 18.0 \mathrm{mmol})$ in DMF (30 mL) was stirred at $80{ }^{\circ} \mathrm{C}$ in an oil bath overnight. The mixture was quenched by water $(20$ $\mathrm{mL})$, extracted with ethyl ether $(6 \times 50 \mathrm{~mL})$. The organic layers were combined, washed with water $(2 \times 30 \mathrm{~mL})$, dried over anhydrous $\mathrm{Na}_{2} \mathrm{SO}_{4}$, and concentrated under reduced pressure. The residue was purified by silica gel column chromatography (petroleum ether/ethyl acetate $=10: 1$ to $2: 1$ ) to afford the desired product S13 as a white solid (1.09 g, 88\% yield).

${ }^{1}$ H NMR $\left(400 \mathrm{MHz} \mathrm{CDCl}_{3}\right) \delta 7.66(\mathrm{~d}, J=7.6 \mathrm{~Hz}, 1 \mathrm{H}), 7.34(\mathrm{t}, J=7.6 \mathrm{~Hz}, 1 \mathrm{H}), 6.98(\mathrm{t}$, $J=7.6 \mathrm{~Hz}, 1 \mathrm{H}), 6.41(\mathrm{~d}, J=8.0 \mathrm{~Hz}, 1 \mathrm{H}), 5.27-5.22(\mathrm{~m}, 1 \mathrm{H}), 4.97(\mathrm{t}, J=6.8 \mathrm{~Hz}, 2 \mathrm{H})$, $4.74(\mathrm{t}, J=6.0 \mathrm{~Hz}, 2 \mathrm{H}), 3.02(\mathrm{q}, J=7.2 \mathrm{~Hz}, 2 \mathrm{H}), 1.15(\mathrm{t}, J=7.2 \mathrm{~Hz}, 3 \mathrm{H}) \mathrm{ppm}$.

${ }^{13} \mathrm{C}$ NMR $\left(100 \mathrm{MHz}, \mathrm{CDCl}_{3}\right) \delta 202.9,155.2,133.1,130.7,128.8,121.5,112.1,77.7,70.6$, 37.1, 8.5 ppm.

HRMS (CI) Calcd for $\mathrm{C}_{12} \mathrm{H}_{13} \mathrm{O}_{3}[\mathrm{M}-\mathrm{H}]^{+}$: 205.0865, Found: 205.0866.

3-(2-(Oxetan-3-yloxy)phenyl)pentan-3-ol (11). At $0{ }^{\circ} \mathrm{C}$, to a solution of 1-(2-(oxetan3-yloxy)phenyl)propan-1-one $\mathbf{S 1 2}(1.09 \mathrm{~g}, 5.3 \mathrm{mmol})$ in THF (20 mL) was added dropwise EtMgBr $\left(8.0 \mathrm{mmol}, 8.0 \mathrm{~mL}, 1 \mathrm{M}\right.$ in THF). After stirring at $0{ }^{\circ} \mathrm{C}$ for $1 \mathrm{~h}$, the 
mixture was slowly quenched with water $(5 \mathrm{~mL})$ and extracted with ethyl acetate $(3 \times 20 \mathrm{~mL})$. The combined organic layers were dried over anhydrous $\mathrm{Na}_{2} \mathrm{SO}_{4}$, and concentrated under reduced pressure. The residue was purified by silica gel column chromatography (petroleum ether/ethyl acetate $=2: 1$ ) to afford the desired product $1 \mathrm{~m}$ as a white solid (374 $\mathrm{mg}, 30 \%$ yield).

${ }^{1} \mathbf{H}$ NMR $\left(400 \mathrm{MHz} \mathrm{CDCl}_{3}\right) \delta 7.43(\mathrm{~d}, J=7.6 \mathrm{~Hz}, 1 \mathrm{H}), 7.15(\mathrm{t}, J=7.6 \mathrm{~Hz}, 1 \mathrm{H}), 6.98(\mathrm{t}$, $J=7.6 \mathrm{~Hz}, 1 \mathrm{H}), 6.34(\mathrm{~d}, J=8.0 \mathrm{~Hz}, 1 \mathrm{H}), 5.26-5.20(\mathrm{~m}, 1 \mathrm{H}), 4.98(\mathrm{t}, J=6.4 \mathrm{~Hz}, 2 \mathrm{H})$, $4.75(\mathrm{t}, J=6.0 \mathrm{~Hz}, 2 \mathrm{H}), 3.09$ (br s, 1H), 2.19-2.10 (m, 2H), 1.88-1.79 (m, 2H), 0.77 (t, J $=7.6 \mathrm{~Hz}, 6 \mathrm{H}) \mathrm{ppm}$.

${ }^{13} \mathrm{C}$ NMR $\left(100 \mathrm{MHz}_{2} \mathrm{CDCl}_{3}\right) \delta 153.5,133.2,128.8,127.9,121.5,111.6,78.0,77.9,70.2$, $32.9,8.3$ ppm.

HRMS (CI) Calcd for $\mathrm{C}_{14} \mathrm{H}_{19} \mathrm{O}_{3}[\mathrm{M}-\mathrm{H}]^{+}: 235.1334$ Found: 235.1340.<smiles>Oc1ccccc1C1(O)CCCC1</smiles>

S13-18

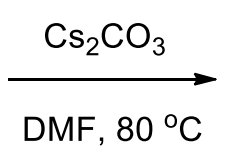

DMF, $80^{\circ} \mathrm{C}$<smiles>OC1(c2ccccc2OC2COC2)CCCC1</smiles>

$1 \mathrm{n}-\mathrm{s}$<smiles>OC1(c2ccccc2OC2COC2)CCC1</smiles>

$1 n$

1-(2-(Oxetan-3-yloxy)phenyl)cyclobutan-1-ol (1n). A mixture of 2-(1-hydroxycyclobutyl)phenol S13 (369 mg, 2.25 mmol), oxetan-3-yl 4-methylbenzenesulfonate (342 mg, $1.5 \mathrm{mmol})$, cesium carbonate (1.48 g, $4.5 \mathrm{mmol})$, and DMF (5 mL) was stirred at $80^{\circ} \mathrm{C}$ in an oil bath overnight. The mixture was quenched by water $(5 \mathrm{~mL})$ and extracted with ethyl ether $(6 \times 20 \mathrm{~mL})$. The organic layers were combined, washed with water $(2 \times 20 \mathrm{~mL})$, dried over anhydrous $\mathrm{Na}_{2} \mathrm{SO}_{4}$, and concentrated under reduced pressure. The residue was purified by silica gel 
column chromatography (petroleum ether/ethyl acetate $=10: 1$ to $2: 1$ ) to give the desired product $1 \mathrm{n}$ as a white solid (322 $\mathrm{mg}, 98 \%$ yield).

${ }^{1} \mathbf{H}$ NMR $\left(400 \mathrm{MHz} \mathrm{CDCl}_{3}\right) \delta 7.34(\mathrm{~d}, J=7.6 \mathrm{~Hz}, 1 \mathrm{H}), 7.18(\mathrm{t}, J=8.0 \mathrm{~Hz}, 1 \mathrm{H}), 7.0(\mathrm{t}, \mathrm{J}$ $=7.6 \mathrm{~Hz}, 1 \mathrm{H}), 6.41(\mathrm{~d}, J=8.0 \mathrm{~Hz}, 1 \mathrm{H}), 5.31-5.26(\mathrm{~m}, 1 \mathrm{H}), 5.00(\mathrm{t}, J=6.8 \mathrm{~Hz}, 2 \mathrm{H}), 4.75$ $(\mathrm{t}, J=6.0 \mathrm{~Hz}, 2 \mathrm{H}), 2.62-2.55(\mathrm{~m}, 2 \mathrm{H}), 2.41-2.38(\mathrm{~m}, 2 \mathrm{H}), 2.14-2.10(\mathrm{~m}, 1 \mathrm{H}), 1.74-1.66$ (m, 1H) ppm.

${ }^{13} \mathrm{C}$ NMR $\left(100 \mathrm{MHz}_{2} \mathrm{CDCl}_{3}\right) \delta 154.3,133.8,128.7,126.4,121.6,111.6,78.0,76.8,70.2$, 35.2, 14.4 ppm.

HRMS (CI) Calcd for $\mathrm{C}_{13} \mathrm{H}_{15} \mathrm{O}_{3}[\mathrm{M}-\mathrm{H}]^{+}:$219.1021, Found: 219.1025.

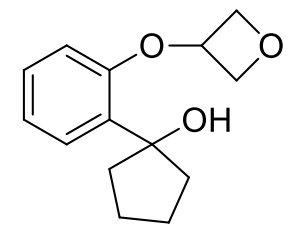

10

1-(2-(Oxetan-3-yloxy)phenyl)cyclopentan-1-ol (10) was prepared from S14 (800 $\mathrm{mg}, 4.49 \mathrm{mmol}$ ) and oxetan-3-yl 4-methyl-benzenesulfonate (570 mg, $2.5 \mathrm{mmol})$ according to the same procedure for the synthesis of 1n (purification by flash column chromatography: petroleum ether/ethyl acetate $=10: 1$ to $3: 1$ ). White solid, $361 \mathrm{mg}, 62 \%$ yield.

${ }^{1} \mathrm{H}$ NMR $\left(400 \mathrm{MHz},\left(\mathrm{CD}_{3}\right)_{2} \mathrm{CO}\right) \delta 7.61(\mathrm{~d}, J=7.6 \mathrm{~Hz}, 1 \mathrm{H}), 7.12(\mathrm{t}, J=7.6 \mathrm{~Hz}, 1 \mathrm{H}), 6.91$ $(\mathrm{t}, J=7.6 \mathrm{~Hz}, 1 \mathrm{H}), 6.50(\mathrm{~d}, J=8.0 \mathrm{~Hz}, 1 \mathrm{H}), 5.32-5.26(\mathrm{~m}, 1 \mathrm{H}), 4.93(\mathrm{t}, J=6.8 \mathrm{~Hz}, 2 \mathrm{H})$, $4.63(\mathrm{t}, J=6.0 \mathrm{~Hz}, 2 \mathrm{H}), 3.67$ (br s, 1H), 2.30-2.24 (m, 2H), 1.94-1.75 (m, 6H) ppm. ${ }^{13} \mathrm{C}$ NMR $\left(100 \mathrm{MHz},\left(\mathrm{CD}_{3}\right)_{2} \mathrm{CO}\right) \delta 153.8,135.8,127.7,126.9,120.8,111.8,81.5,77.3$, $70.3,40.0,24.6 \mathrm{ppm}$.

HRMS (CI) Calcd for $\mathrm{C}_{14} \mathrm{H}_{17} \mathrm{O}_{3}[\mathrm{M}-\mathrm{H}]^{+}:$233.1178, Found: 233.1190. 
<smiles>OC1(c2ccccc2OC2COC2)CCCCC1</smiles>

$1 \mathrm{p}$

1-(2-(Oxetan-3-yloxy)phenyl)cyclohexan-1-ol (1p) was prepared from S15 (692 $\mathrm{mg}, 3.6 \mathrm{mmol}$ ) and oxetan-3-yl 4-methyl-benzenesulfonate $(685 \mathrm{mg}, 3.0 \mathrm{mmol})$ according to the same procedure for the synthesis of 1 n (purification by flash column chromatography: petroleum ether/ethyl acetate $=10: 1$ to $3: 1$ ). White solid, $710 \mathrm{mg}, 95 \%$ yield.

${ }^{1} \mathrm{H}$ NMR $\left(400 \mathrm{MHz}, \mathrm{CDCl}_{3}\right) \delta 7.43(\mathrm{~d}, J=7.6 \mathrm{~Hz}, 1 \mathrm{H}), 7.16(\mathrm{t}, J=7.6 \mathrm{~Hz}, 1 \mathrm{H}), 6.98(\mathrm{t}$, $J=6.8 \mathrm{~Hz}, 1 \mathrm{H}), 6.38(\mathrm{~d}, J=8.4 \mathrm{~Hz}, 1 \mathrm{H}), 5.28-5.24(\mathrm{~m}, 1 \mathrm{H}), 5.0(\mathrm{t}, J=6.8 \mathrm{~Hz}, 2 \mathrm{H}), 4.77$ $(\mathrm{t}, J=5.6 \mathrm{~Hz}, 2 \mathrm{H}), 3.30$ (br s, $1 \mathrm{H}), 1.97-1.72(\mathrm{~m}, 7 \mathrm{H}), 1.63-1.60(\mathrm{~m}, 2 \mathrm{H}), 1.31-1.22(\mathrm{~m}$, 1H) ppm.

${ }^{13} \mathrm{C}$ NMR $\left(100 \mathrm{MHz}, \mathrm{CDCl}_{3}\right) \delta 153.9,136.8,128.0,126.5,121.8,111.9,77.9,73.1,70.2$, 36.5, 25.8, 22.0 ppm.

HRMS (CI) Calcd for $\mathrm{C}_{15} \mathrm{H}_{19} \mathrm{O}_{3}[\mathrm{M}-\mathrm{H}]^{+}:$247.1334, Found: 247.1350.

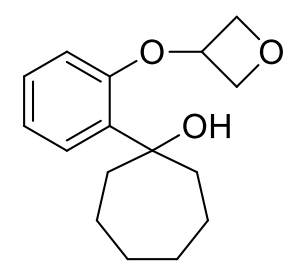

$1 \mathrm{q}$

1-(2-(Oxetan-3-yloxy)phenyl)cycloheptan-1-ol (1q) was prepared from S16 (1.55 g, $7.5 \mathrm{mmol})$ and oxetan-3-yl 4-methyl-benzenesulfonate (1.14 g, $5.0 \mathrm{mmol})$ according to the same procedure for the synthesis of 1n (purification by flash column chromatography: petroleum ether/ethyl acetate $=10: 1$ to $3: 1$ ). White solid, $799 \mathrm{mg}, 61 \%$ yield

${ }^{1} \mathrm{H}$ NMR $\left(400 \mathrm{MHz}^{\mathrm{CDCl}} 3\right) \delta 7.40(\mathrm{~d}, J=8.0 \mathrm{~Hz}, 1 \mathrm{H}), 7.15(\mathrm{t}, J=7.6 \mathrm{~Hz}, 1 \mathrm{H}), 6.98(\mathrm{t}$, $J=7.6 \mathrm{~Hz}, 1 \mathrm{H}), 6.38(\mathrm{~d}, J=8.0 \mathrm{~Hz}, 1 \mathrm{H}), 5.31-5.26(\mathrm{~m}, 1 \mathrm{H}), 5.01(\mathrm{t}, J=6.4 \mathrm{~Hz}, 2 \mathrm{H})$, 
$4.80(\mathrm{t}, J=4.8 \mathrm{~Hz}, 2 \mathrm{H}), 3.64(\mathrm{br} \mathrm{s}, 1 \mathrm{H}), 2.31-2.18(\mathrm{~m}, 2 \mathrm{H}), 2.07\left(\mathrm{dd}, J_{1}=14.4 \mathrm{~Hz}, J_{2}=\right.$ 8.0 Hz, 2H), 1.91-1.82 (m, 2H), 1.76-1.69 (m, 2H), 1.59-1.54 (m, 4H) ppm.

${ }^{13} \mathrm{C}$ NMR $\left(100 \mathrm{MHz}, \mathrm{CDCl}_{3}\right) \delta 153.8,138.0,128.9,126.6,121.9,112.1,78.0,77.5,70.5$, 40.8, 29.8, 22.8 ppm.

HRMS (CI) Calcd for $\mathrm{C}_{16} \mathrm{H}_{21} \mathrm{O}_{3}[\mathrm{M}-\mathrm{H}]^{+}:$261.1491, Found: 261.1486.<smiles>OC1(c2ccccc2OC2COC2)CCSCC1</smiles>

4-(2-(Oxetan-3-yloxy)phenyl)tetrahydro-2H-thiopyran-4-ol (1r) was prepared from S17 (581 mg, $2.76 \mathrm{mmol}$ ) and oxetan-3-yl 4-methyl-benzenesulfonate (525 mg, $2.3 \mathrm{mmol}$ ) according to the same procedure for the synthesis of $\mathbf{1 n}$ (purification by flash column chromatography: petroleum ether/ethyl acetate $=10: 1$ to $3: 1$ ). White solid, $508 \mathrm{mg}$, 82\% yield.

${ }^{1} \mathrm{H}$ NMR $\left(400 \mathrm{MHz}, \mathrm{CDCl}_{3}\right) \delta 7.36\left(\mathrm{dd}, J_{1}=7.6 \mathrm{~Hz}, J_{2}=0.8 \mathrm{~Hz}, 1 \mathrm{H}\right), 7.19(\mathrm{t}, J=6.8 \mathrm{~Hz}$, 1H), $7.01(\mathrm{t}, J=7.6 \mathrm{~Hz}, 1 \mathrm{H}), 6.39(\mathrm{~d}, J=8.0 \mathrm{~Hz}, 1 \mathrm{H}), 5.31-5.25(\mathrm{~m}, 1 \mathrm{H}), 5.01(\mathrm{t}, J=6.8$ $\mathrm{Hz}, 2 \mathrm{H}), 4.78(\mathrm{t}, J=5.6 \mathrm{~Hz}, 2 \mathrm{H}), 3.67(\mathrm{br} \mathrm{s}, 1 \mathrm{H}), 3.30\left(\mathrm{td}, J_{1}=12.0 \mathrm{~Hz}, J_{2}=4.8 \mathrm{~Hz}, 2 \mathrm{H}\right)$, $2.44(\mathrm{~d}, J=13.6 \mathrm{~Hz}, 2 \mathrm{H}), 2.34-2.22(\mathrm{~m}, 4 \mathrm{H}) \mathrm{ppm}$.

${ }^{13} \mathrm{C}$ NMR $\left(100 \mathrm{MHz}, \mathrm{CDCl}_{3}\right) \delta 153.7,136.1,128.5,126.4,122.2,112.1,77.8,71.9,70.5$, 37.6, $24.1 \mathrm{ppm}$.

HRMS (CI) Calcd for $\mathrm{C}_{14} \mathrm{H}_{17} \mathrm{O}_{3} \mathrm{~S}[\mathrm{M}-\mathrm{H}]^{+}:$265.0898, Found: 265.0890.<smiles>OC1(c2ccccc2OC2COC2)CCOCC1</smiles> 
4-(2-(Oxetan-3-yloxy)phenyl)tetrahydro-2H-pyran-4-ol (1s) was prepared from S18 (430 mg, $2.22 \mathrm{mmol}$ ) and oxetan-3-yl 4-methyl-benzenesulfonate (422 mg, 1.85 mmol) according to the same procedure for the synthesis of 1n (purification by flash column chromatography: petroleum ether/ethyl acetate $=10: 1$ to $3: 1$ ). White solid, $256 \mathrm{mg}$, 77\% yield

${ }^{1} \mathbf{H}$ NMR $\left(400 \mathrm{MHz}, \mathrm{CDCl}_{3}\right) \delta 7.35\left(\mathrm{dd}, J_{1}=8.0 \mathrm{~Hz}, J_{2}=1.6 \mathrm{~Hz}, 1 \mathrm{H}\right), 7.21\left(\mathrm{td}, J_{1}=7.6\right.$ $\left.\mathrm{Hz}, J_{2}=1.2 \mathrm{~Hz}, 1 \mathrm{H}\right), 7.02(\mathrm{t}, J=7.6 \mathrm{~Hz}, 1 \mathrm{H}), 6.42(\mathrm{~d}, J=8.0 \mathrm{~Hz}, 1 \mathrm{H}), 5.33-5.27(\mathrm{~m}$, $1 \mathrm{H}), 5.02(\mathrm{t}, J=7.2 \mathrm{~Hz}, 2 \mathrm{H}), 4.77(\mathrm{t}, J=5.2 \mathrm{~Hz}, 2 \mathrm{H}), 4.05-3.99(\mathrm{~m}, 2 \mathrm{H}), 3.86\left(\mathrm{dd}, J_{1}=\right.$ $\left.11.2 \mathrm{~Hz}, J_{2}=4.8 \mathrm{~Hz}, 2 \mathrm{H}\right), 3.75(\mathrm{br} \mathrm{s}, 1 \mathrm{H}), 2.23\left(\mathrm{td}, J_{1}=13.2 \mathrm{~Hz}, J_{2}=5.4 \mathrm{~Hz}, 2 \mathrm{H}\right), 1.95$ $(\mathrm{d}, J=12.4 \mathrm{~Hz}, 2 \mathrm{H})$ ppm.

${ }^{13} \mathrm{C}$ NMR $\left(100 \mathrm{MHz}, \mathrm{CDCl}_{3}\right) \delta 154.2,135.2,128.6,126.4,122.2,112.0,77.8,70.7,70.5$, 63.8, 37.0 ppm.

HRMS (CI) Calcd for $\mathrm{C}_{14} \mathrm{H}_{17} \mathrm{O}_{4}[\mathrm{M}-\mathrm{H}]^{+}:$249.1127, Found: 249.1120.<smiles>COC(=O)c1ccccc1SC1COC1</smiles>

S19

Methyl 2-(oxetan-3-ylthio)benzoate (S19) was prepared from methyl 2mercaptobenzoate $(1.26 \mathrm{~g}, 7.5 \mathrm{mmol})$ and oxetan-3-yl 4-methyl-benzenesulfonate (1.14 g, $5.0 \mathrm{mmol})$ according to the same procedure for the synthesis of S19 (purification by flash column chromatography: petroleum ether/ethyl acetate $=5: 1$ to $2: 1)$. Colorless oil, $455 \mathrm{mg}, 41 \%$ yield.

${ }^{1}$ H NMR $\left(400 \mathrm{MHz}, \mathrm{CDCl}_{3}\right) \delta 8.00(\mathrm{~d}, J=7.6 \mathrm{~Hz}, 1 \mathrm{H}), 7.41(\mathrm{t}, J=7.6 \mathrm{~Hz}, 1 \mathrm{H}), 7.18(\mathrm{t}$, $J=7.6 \mathrm{~Hz}, 1 \mathrm{H}), 6.79(\mathrm{~d}, J=8.0 \mathrm{~Hz}, 1 \mathrm{H}), 5.14(\mathrm{t}, J=6.8 \mathrm{~Hz}, 2 \mathrm{H}), 4.67(\mathrm{t}, J=6.4 \mathrm{~Hz}, 2 \mathrm{H})$, 4.56-4.50 (m, 1H), $3.92(\mathrm{~s}, 3 \mathrm{H}) \mathrm{ppm}$.

${ }^{13} \mathrm{C}$ NMR $\left(100 \mathrm{MHz}, \mathrm{CDCl}_{3}\right) \delta 166.7,140.6,132.8,131.7,127.2,125.1,124.5,77.5,52.2$, $37.4 \mathrm{ppm}$.

HRMS (CI) Calcd for $\mathrm{C}_{11} \mathrm{H}_{11} \mathrm{O}_{3} \mathrm{~S}[\mathrm{M}-\mathrm{H}]^{+}$: 223.0429, Found: 223.0431. 


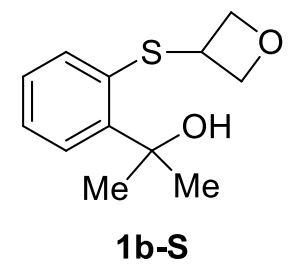

2-(2-(Oxetan-3-ylthio) phenyl)propan-2-ol (1b-S) was prepared from S19 ( $910 \mathrm{mg}$, $4.06 \mathrm{mmol})$ and $\mathrm{MeMgBr}(12.18 \mathrm{mmol}, 4.06 \mathrm{~mL}, 3 \mathrm{M}$ in THF) according to the same procedure for the synthesis of $\mathbf{1 f}$ (purification by flash column chromatography: petroleum ether/ethyl acetate $=5: 1$ to 3:1). Colorless oil, $710 \mathrm{mg}$, 78\% yield.

${ }^{1} \mathrm{H}$ NMR $\left(400 \mathrm{MHz}, \mathrm{CDCl}_{3}\right) \delta 7.44(\mathrm{t}, J=3.2 \mathrm{~Hz}, 1 \mathrm{H}), 7.19-7.17(\mathrm{~m}, 2 \mathrm{H}), 7.0(\mathrm{~d}, J=$ $4.8 \mathrm{~Hz}, 1 \mathrm{H}), 5.02(\mathrm{t}, J=6.8 \mathrm{~Hz}, 2 \mathrm{H}), 4.69(\mathrm{t}, J=6.4 \mathrm{~Hz}, 2 \mathrm{H}), 4.50-4.43(\mathrm{~m}, 1 \mathrm{H}), 3.54$ (br s, 1H), 1.68 (s, 6H) ppm.

${ }^{13} \mathrm{C}$ NMR (100 MHz, $\left.\mathrm{CDCl}_{3}\right) \delta 147.4,132.7,130.9,127.7,126.8,126.2,78.0,73.8,40.7$, $30.6 \mathrm{ppm}$.

HRMS (CI) Calcd for $\mathrm{C}_{12} \mathrm{H}_{15} \mathrm{O}_{2} \mathrm{~S}$ [M - H]+: 223.0793, Found: 223.0791.

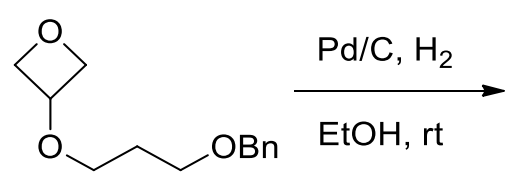

S20

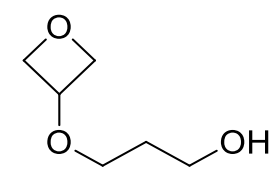

3

3-(Oxetan-3-yloxy)propan-1-ol (3) was prepared according to the reported procedure. ${ }^{3}$ Colorless oil, $90 \%$ yield.

${ }^{1} \mathrm{H} \mathrm{NMR}\left(400 \mathrm{MHz}, \mathrm{CDCl}_{3}\right) \delta 4.71(\mathrm{t}, J=6.8 \mathrm{~Hz}, 2 \mathrm{H}), 4.54(\mathrm{t}, J=5.2 \mathrm{~Hz}, 2 \mathrm{H}), 4.49(\mathrm{q}$, $J=5.6 \mathrm{~Hz}, 1 \mathrm{H}), 3.67(\mathrm{t}, J=6.0 \mathrm{~Hz}, 2 \mathrm{H}), 3.44(\mathrm{t}, J=6.0 \mathrm{~Hz}, 2 \mathrm{H}), 2.68(\mathrm{br} \mathrm{s}, 1 \mathrm{H}), 1.79$ (m, 2H) ppm.

${ }^{13} \mathrm{C}$ NMR $\left(100 \mathrm{MHz}, \mathrm{CDCl}_{3}\right) \delta 78.7,72.4,66.7,60.4,32.3 \mathrm{ppm}$.

3 W. Z. Ong, P. W. Nowak, B. C. Askew, J. Kim, PCT Int. Appl. (2014), WO 2014127214 A1 20140821. 
<smiles>CCOC(=O)C=C1COC1</smiles>

S21

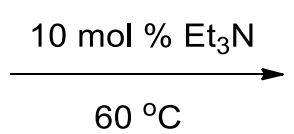

$\mathrm{OH}_{\mathrm{OH}}$

Ethyl 2-(3-((3-hydroxypropyl)thio)oxetan-3-yl)acetate (5) A mixture of ethyl 2(oxetan-3-ylidene)acetate S21 ${ }^{4}$ (711 mg, 5.0 mmol), 3-mercaptopropan-1-ol (461 $\mathrm{mg}, 5.0 \mathrm{mmol})$, and triethylamine $(70 \mu \mathrm{L}, 0.5 \mathrm{mmol})$ was stirred at $60{ }^{\circ} \mathrm{C}$ in an oil bath for $12 \mathrm{~h}$. The mixture was directly purified by silica gel column chromatography (petroleum ether/ethyl acetate $=2: 1$ to 1:1) to afford the desired product 5 as colorless oil (997 $\mathrm{mg}, 85 \%$ yield).

${ }^{1} \mathrm{H}$ NMR $\left(400 \mathrm{MHz}, \mathrm{CDCl}_{3}\right) \delta 4.76(\mathrm{~d}, J=6.8 \mathrm{~Hz}, 2 \mathrm{H}), 4.64(\mathrm{~d}, J=6.8 \mathrm{~Hz}, 2 \mathrm{H}), 4.12$ $(\mathrm{q}, J=7.2 \mathrm{~Hz}, 2 \mathrm{H}), 3.68(\mathrm{t}, J=6.0 \mathrm{~Hz}, 2 \mathrm{H}), 2.94(\mathrm{~s}, 2 \mathrm{H}), 2.70(\mathrm{t}, J=7.2 \mathrm{~Hz}, 2 \mathrm{H}), 2.39$ (br s, 1H), 1.82-1.75 (m, 2H), $1.22(\mathrm{t}, J=7.2 \mathrm{~Hz}, 3 \mathrm{H}) \mathrm{ppm}$.

${ }^{13} \mathrm{C}$ NMR (100 MHz, $\left.\mathrm{CDCl}_{3}\right) \delta 170.1,82.0,61.0,60.9,47.1,42.4,32.0,25.5,14.2 \mathrm{ppm}$. HRMS (CI) Calcd for $\mathrm{C}_{10} \mathrm{H}_{17} \mathrm{O}_{4} \mathrm{~S}$ [M - H] $]^{+}$233.0848, Found: 233.0840.

${ }^{4}$ G. Wuitschik, M. Rogers-Evans, K. Müller, H. Fischer, B. Wagner, F. Schuler, L. Polonchuk, E. M. Carreira, Angew. Chem. Int. Ed. 2006, 45, 7736-7739. 


\section{Organocatalytic Intramolecular Openings of Oxetanes}<smiles>[X]c1c#[R]ccc1C([R])O</smiles>

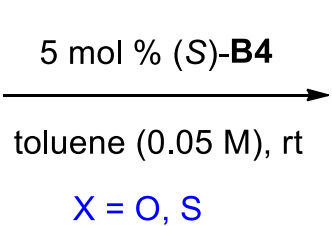

$X=0, S$<smiles>[R][R]C1OC[C@@H](CO)[X]c2cc[R]:[X]c21</smiles>

2

General Procedure. To a solution of oxetane $1(0.30 \mathrm{mmol})$ in toluene $(6.0 \mathrm{~mL}, c=$ $0.05 \mathrm{M}$ ) was added catalyst $(S)-\mathbf{B} 4(5 \mathrm{~mol} \%, 0.015 \mathrm{mmol}, 10.7 \mathrm{mg})$. After stirring at room temperature upon completion, the mixture was directly purified by silica gel column chromatography to give the desired product 2.<smiles>CC1(C)OC[C@@H](CO)Oc2ccccc21</smiles>

(R)-(5,5-Dimethyl-3,5-dihydro-2H-benzo[e][1,4]dioxepin-2-yl)methanol (2b) was prepared from (2-(oxetan-3-yloxy)phenyl)propan-2-ol $1 \mathbf{b}(62.5 \mathrm{mg}, 0.30 \mathrm{mmol})$ according to the general procedure ( $\mathrm{rt}, 16 \mathrm{~h}$; purification by flash column chromatography: petroleum ether/ethyl acetate $=10: 3,59.3 \mathrm{mg}$, white solid, $95 \%$ yield).

$[\alpha]_{\mathrm{D}^{10}}:+4.3\left(c=0.5, \mathrm{CHCl}_{3}\right) .91 \%$ ee, HPLC analysis of the product: Daicel CHIRALCEL OD-H column; 5\% $i-\mathrm{PrOH}$ in hexanes; $1.0 \mathrm{~mL} / \mathrm{min} ; 35{ }^{\circ} \mathrm{C}$; retention times: $12.3 \mathrm{~min}$ (minor), $13.6 \mathrm{~min}$ (major).

${ }^{1} \mathrm{H}$ NMR $\left(400 \mathrm{MHz}, \mathrm{CDCl}_{3}\right) \delta 7.25(\mathrm{~d}, J=6.4 \mathrm{~Hz}, 1 \mathrm{H}), 7.20(\mathrm{t}, J=7.6 \mathrm{~Hz}, 1 \mathrm{H}), 7.09$ $7.02(\mathrm{~m}, 2 \mathrm{H}), 4.05-3.94(\mathrm{~m}, 2 \mathrm{H}), 3.86\left(\mathrm{dd}, J_{1}=12.4 \mathrm{~Hz}, J_{2}=2.0 \mathrm{~Hz}, 1 \mathrm{H}\right), 3.80-3.71(\mathrm{~m}$, 2H), 2.19 (br s, 1H), 1.67 (s, 3H), 1.61 (s, 3H) ppm.

${ }^{13} \mathrm{C}$ NMR $\left(100 \mathrm{MHz}, \mathrm{CDCl}_{3}\right) \delta 155.7,138.8,128.5,126.5,124.0,122.3,82.0,78.1,64.6$, 62.6, 28.7, $27.0 \mathrm{ppm}$. 
HRMS (CI) Calcd for $\mathrm{C}_{12} \mathrm{H}_{15} \mathrm{O}_{3}[\mathrm{M}-\mathrm{H}]^{+}$: 207.1021, Found: 207.1014.<smiles>CC1(C)OC[C@H](CO)Oc2ccc(F)cc21</smiles>

(R)-(7-Fluoro-5,5-dimethyl-3,5-dihydro-2H-benzo[e][1,4]dioxepin-2-yl)methanol (2c) was prepared from 2-(5-fluoro-2-(oxetan-3-yloxy)phenyl)propan-2-ol 1c (67.9 $\mathrm{mg}, 0.30 \mathrm{mmol}$ ) according to the general procedure ( $\mathrm{rt}, 24 \mathrm{~h}$; purification by flash column chromatography: petroleum ether/ethyl acetate $=2: 1,63.6 \mathrm{mg}$, white solid, $94 \%$ yield).

$[\alpha]_{\mathrm{D}^{10}}:+11.5\left(c=0.5, \mathrm{CHCl}_{3}\right) .87 \%$ ee, HPLC analysis of the product: Daicel CHIRALCEL OD-H column; 5\% $i-\mathrm{PrOH}$ in hexanes; $1.0 \mathrm{~mL} / \mathrm{min} ; 35^{\circ} \mathrm{C}$; retention times: $10.1 \mathrm{~min}$ (minor), $11.9 \mathrm{~min}$ (major).

${ }^{1} \mathbf{H}$ NMR $\left(400 \mathrm{MHz} \mathrm{CDCl}_{3}\right) \delta$ 6.98-6.92 (m, 2H), $6.84(\mathrm{t}, J=8.0 \mathrm{~Hz}, 1 \mathrm{H}), 4.03-3.98$ (m, 1H), 3.90-3.82 (m, 2H), 3.76-3.67 (m, 2H), 2.42 (br s, 1H), 1.62 (s, 3H), 1.58 (s, 3H) ppm.

${ }^{13} \mathrm{C}$ NMR (100 MHz, $\left.\mathrm{CDCl}_{3}\right) \delta 158.9$ (d, $\left.J=240.2 \mathrm{~Hz}\right), 151.9$ (d, J = 2.4 Hz), 140.8 (d, $J=6.4 \mathrm{~Hz}), 123.4(\mathrm{~d}, J=8.4 \mathrm{~Hz}), 114.6(\mathrm{~d}, J=22.5 \mathrm{~Hz}), 113.5(\mathrm{~d}, J=24.2 \mathrm{~Hz}), 82.2$, $77.9,64.9,62.6,28.6,26.7 \mathrm{ppm}$.

HRMS (CI) Calcd for $\mathrm{C}_{12} \mathrm{H}_{15} \mathrm{FNaO}_{3}[\mathrm{M}+\mathrm{Na}]^{+}:$249.0903, Found: 249.0978.<smiles>CC1(C)OC[C@H](CO)Oc2ccc(Cl)cc21</smiles>

2d

(R)-(7-Chloro-5,5-dimethyl-3,5-dihydro-2H-benzo[e][1,4]dioxepin-2-yl)-

methanol (2d) was prepared from 2-(5-chloro-2-(oxetan-3-yloxy)phenyl)propan-2ol $1 \mathrm{~d}(72.8 \mathrm{mg}, 0.30 \mathrm{mmol})$ according to the general procedure ( $\mathrm{rt}, 24 \mathrm{~h}$; purification 
by flash column chromatography: petroleum ether/ethyl acetate $=2: 1,72.5 \mathrm{mg}$, white solid, 99\% yield).

$[\alpha]_{\mathrm{D}^{10}}:+4.6\left(c=0.5, \mathrm{CHCl}_{3}\right) .87 \%$ ee, HPLC analysis of the product: Daicel CHIRALCEL OD-H column; 5\% $i-\mathrm{PrOH}$ in hexanes; $1.0 \mathrm{~mL} / \mathrm{min} ; 35^{\circ} \mathrm{C}$; retention times: 9.9 min (minor), $11.5 \min$ (major).

${ }^{1} \mathrm{H}$ NMR $\left(400 \mathrm{MHz}, \mathrm{CDCl}_{3}\right) \delta 7.19(\mathrm{~s}, 1 \mathrm{H}), 7.12(\mathrm{~d}, J=8.4 \mathrm{~Hz}, 1 \mathrm{H}), 6.95(\mathrm{~d}, J=8.4 \mathrm{~Hz}$, 1H), 4.03-3.92 (m, 2H), $3.84(\mathrm{~d}, J=13.2 \mathrm{~Hz}, 1 \mathrm{H}), 3.76-3.68(\mathrm{~m}, 2 \mathrm{H}), 2.37$ (br s, 1H), $1.63(\mathrm{~s}, 3 \mathrm{H}), 1.57(\mathrm{~s}, 3 \mathrm{H}) \mathrm{ppm}$.

${ }^{13} \mathrm{C}$ NMR (100 MHz, $\left.\mathrm{CDCl}_{3}\right) \delta 154.6,140.7,129.1,128.4,126.7,123.8,82.3,77.9,64.7$, 62.6, 28.7, $26.8 \mathrm{ppm}$.

HRMS (CI) Calcd for $\mathrm{C}_{12} \mathrm{H}_{15} \mathrm{ClNaO}_{3}[\mathrm{M}+\mathrm{Na}]^{+}:$265.0607, Found: 265.0658.<smiles>CC1(C)OC[C@H](CO)Oc2ccc(Br)cc21</smiles>

$2 e$

(R)-(7-Bromo-5,5-dimethyl-3,5-dihydro-2H-benzo[e][1,4]dioxepin-2-yl)methanol (2e) was prepared from 2-(5-bromo-2-(oxetan-3-yloxy)phenyl)propan-2-ol 1e (86.2 $\mathrm{mg}, 0.30 \mathrm{mmol}$ ) according to the general procedure (rt, $24 \mathrm{~h}$; purification by flash column chromatography: petroleum ether/ethyl acetate $=2: 1,84.9 \mathrm{mg}$, white solid, $98 \%$ yield).

$[\alpha]_{\mathrm{D}^{10}}:+6.0\left(c=0.5, \mathrm{CHCl}_{3}\right) .88 \%$ ee, HPLC analysis of the product: Daicel CHIRALCEL OD-H column; $5 \% i-\mathrm{PrOH}$ in hexanes; $1.0 \mathrm{~mL} / \mathrm{min} ; 35^{\circ} \mathrm{C}$; retention times: $10.1 \mathrm{~min}$ (minor), $11.4 \mathrm{~min}$ (major).

${ }^{1} \mathrm{H}$ NMR $\left(400 \mathrm{MHz}, \mathrm{CDCl}_{3}\right) \delta 7.26(\mathrm{~s}, 1 \mathrm{H}), 7.20(\mathrm{~d}, J=6.4 \mathrm{~Hz}, 1 \mathrm{H}), 6.82(\mathrm{~d}, J=8.4 \mathrm{~Hz}$, 1H), 3.97-3.84 (m, 2H), $3.76(\mathrm{~d}, J=14.0 \mathrm{~Hz}, 1 \mathrm{H}), 3.69-3.60(\mathrm{~m}, 2 \mathrm{H}), 2.45$ (br s, 1H), $1.55(\mathrm{~s}, 3 \mathrm{H}), 1.49(\mathrm{~s}, 3 \mathrm{H}) \mathrm{ppm}$. 
${ }^{13} \mathrm{C}$ NMR $\left(100 \mathrm{MHz}_{2} \mathrm{CDCl}_{3}\right) \delta 155.1,141.1,131.4,129.6,124.2,116.8,82.3,77.9,64.7$, 62.5, 28.7, $26.8 \mathrm{ppm}$.

HRMS (CI) Calcd for $\mathrm{C}_{12} \mathrm{H}_{15} \mathrm{BrNaO}_{3}[\mathrm{M}+\mathrm{Na}]^{+}:$311.0082, Found: 311.0143 .<smiles>CC1(C)OC[C@@H](CO)Oc2cc(Cl)ccc21</smiles>

(R)-(8-Chloro-5,5-dimethyl-3,5-dihydro-2H-benzo[e][1,4]dioxepin-2-

yl)methanol (2f) was prepared from 2-(4-chloro-2-(oxetan-3yloxy)phenyl)propan-2-ol $\mathbf{1 f}(72.8 \mathrm{mg}, 0.30 \mathrm{mmol})$ according to the general procedure ( $\mathrm{rt}, 36 \mathrm{~h}$; purification by flash column chromatography: petroleum ether/ethyl acetate $=2: 1,71.1 \mathrm{mg}$, white solid, 98\% yield).

$[\alpha]_{\mathrm{D}}^{10}:+28.1\left(c=0.5, \mathrm{CHCl}_{3}\right) .92 \%$ ee, HPLC analysis of the product: Daicel CHIRALCEL OD-H column; $5 \%$ i-PrOH in hexanes; $1.0 \mathrm{~mL} / \mathrm{min} ; 35{ }^{\circ} \mathrm{C}$; retention times: $9.5 \mathrm{~min}$ (minor), $11.1 \mathrm{~min}$ (major).

${ }^{1}$ H NMR $\left(400 \mathrm{MHz}, \mathrm{CDCl}_{3}\right) \delta 7.15(\mathrm{~d}, J=8.0 \mathrm{~Hz}, 1 \mathrm{H}), 7.02(\mathrm{~d}, J=9.6 \mathrm{~Hz}, 2 \mathrm{H}), 4.05-$ $3.91(\mathrm{~m}, 2 \mathrm{H}), 3.85(\mathrm{~d}, J=13.2 \mathrm{~Hz}, 1 \mathrm{H}), 3.77-3.69(\mathrm{~m}, 2 \mathrm{H}), 2.57$ (br s, 1H), $1.63(\mathrm{~s}, 3 \mathrm{H})$, $1.56(\mathrm{~s}, 3 \mathrm{H}) \mathrm{ppm}$.

${ }^{13} \mathrm{C}$ NMR $\left(100 \mathrm{MHz}, \mathrm{CDCl}_{3}\right) \delta 156.7,137.6,133.3,127.6,124.0,122.7,82.6,77.8,64.7$, $62.5,28.8,26.9$ ppm.

HRMS (CI) Calcd for $\mathrm{C}_{12} \mathrm{H}_{15} \mathrm{ClKO}_{3}[\mathrm{M}+\mathrm{K}]^{+}:$281.0347, Found: 281.1232.<smiles>Cc1ccc2c(c1)O[C@H](CO)COC2(C)C</smiles>

$2 \mathrm{~g}$

(R)-(5,5,8-Trimethyl-3,5-dihydro-2H-benzo[e][1,4]dioxepin-2-yl)methanol (2g) was prepared from 2-(4-methyl-2-(oxetan-3-yloxy)phenyl)propan-2-ol 1g (66.7 mg, 
$0.30 \mathrm{mmol}$ ) according to the general procedure ( $\mathrm{rt}, 24 \mathrm{~h}$, purification by flash column chromatography: petroleum ether/ethyl acetate $=10: 3,63.2 \mathrm{mg}$, white solid, $95 \%$ yield).

$[\alpha]_{D^{10}}:+14.4\left(c=0.5, \mathrm{CHCl}_{3}\right) .93 \%$ ee, HPLC analysis of the product: Daicel CHIRALCEL OD-H column; $5 \% i$-PrOH in hexanes; $1.0 \mathrm{~mL} / \mathrm{min} ; 35^{\circ} \mathrm{C}$; retention times: $9.7 \mathrm{~min}$ (minor), $12.8 \mathrm{~min}$ (major).

${ }^{1}$ H NMR $\left(400 \mathrm{MHz}, \mathrm{CDCl}_{3}\right) \delta 7.12(\mathrm{~d}, J=7.6 \mathrm{~Hz}, 1 \mathrm{H}), 6.86(\mathrm{~d}, J=8.0 \mathrm{~Hz}, 2 \mathrm{H}), 4.03-$ $3.92(\mathrm{~m}, 2 \mathrm{H}), 3.84(\mathrm{~d}, J=12.4 \mathrm{~Hz}, 1 \mathrm{H}), 3.78-3.70(\mathrm{~m}, 2 \mathrm{H}), 2.51$ (br s, 1H), $2.29(\mathrm{~s}, 3 \mathrm{H})$, $1.65(\mathrm{~s}, 3 \mathrm{H}), 1.59(\mathrm{~s}, 3 \mathrm{H}) \mathrm{ppm}$.

${ }^{13} \mathrm{C}$ NMR $\left(100 \mathrm{MHz}, \mathrm{CDCl}_{3}\right) \delta 155.6,138.8,135.8,126.5,124.7,122.9,82.1,78.1,64.7$, 62.8, 28.8, 27.2, 20.7 ppm.

HRMS (CI) Calcd for $\mathrm{C}_{13} \mathrm{H}_{18} \mathrm{NaO}_{3}[\mathrm{M}+\mathrm{Na}]^{+}:$245.1154, Found: 245.1243.<smiles>COc1ccc2c(c1)C(C)(C)OC[C@@H](CO)O2</smiles>

$2 h$

(R)-(7-Methoxy-5,5-dimethyl-3,5-dihydro-2H-benzo[e][1,4]dioxepin-2-

yl)methanol (2h) was prepared from 2-(5-methoxy-2-(oxetan-3yloxy)phenyl)propan-2-ol $\mathbf{1 h}(71.5 \mathrm{mg}, 0.30 \mathrm{mmol})$ according to the general procedure (rt, $16 \mathrm{~h}$; purification by flash column chromatography: petroleum ether/ethyl acetate $=10: 3,70.4 \mathrm{mg}$, colorless oil, $98 \%$ yield).

$[\alpha]_{\mathrm{D}^{10}}:-4.9\left(c=0.5, \mathrm{CHCl}_{3}\right) .88 \%$ ee, HPLC analysis of the product: Daicel CHIRALPAK AD-H column; $10 \% i$-PrOH in hexanes; $1.0 \mathrm{~mL} / \mathrm{min} ; 35^{\circ} \mathrm{C}$; retention times: $9.4 \mathrm{~min}$ (major), $10.0 \mathrm{~min}$ (minor).

${ }^{1} \mathrm{H}$ NMR $\left(400 \mathrm{MHz}, \mathrm{CDCl}_{3}\right) \delta 6.93(\mathrm{~d}, J=8.4 \mathrm{~Hz}, 1 \mathrm{H}), 6.76(\mathrm{~s}, 1 \mathrm{H}), 6.66(\mathrm{~d}, J=8.4 \mathrm{~Hz}$, 1H), 3.98-3.85 (m, 2H), $3.81(\mathrm{~d}, J=12.8 \mathrm{~Hz}, 1 \mathrm{H}), 3.74(\mathrm{~s}, 3 \mathrm{H}), 3.72-3.63(\mathrm{~m}, 2 \mathrm{H}), 2.68$ (br s, 1H), $1.61(\mathrm{~s}, 3 \mathrm{H}), 1.57(\mathrm{~s}, 3 \mathrm{H}) \mathrm{ppm}$. 
${ }^{13} \mathrm{C}$ NMR $\left(100 \mathrm{MHz}_{2} \mathrm{CDCl}_{3}\right) \delta 155.7,149.4,140.0,122.9,113.1,112.1,82.0,78.2,64.8$, 62.5, 55.6, 28.7, 26.9 ppm.

HRMS (CI) Calcd for $\mathrm{C}_{13} \mathrm{H}_{18} \mathrm{NaO}_{4}[\mathrm{M}+\mathrm{Na}]^{+}:$261.1103, Found: 261.1170.<smiles>CC1(C)OC[C@@H](CO)Oc2ccc(OCc3ccccc3)cc21</smiles>

(R)-(7-(Benzyloxy)-5,5-dimethyl-3,5-dihydro-2H-benzo[e][1,4]dioxepin-2-yl)-

methanol (2i) was prepared from 2-(5-(benzyloxy)-2-(oxetan-3-yloxy)phenyl)propan-2-ol $1 \mathbf{i}(94.3 \mathrm{mg}, 0.30 \mathrm{mmol})$ according to the general procedure (rt, $20 \mathrm{~h}$; purification by flash column chromatography: petroleum ether/ethyl acetate $=10: 3$, $86.6 \mathrm{mg}$, white solid, 92\% yield).

$[\alpha]_{\mathrm{D}^{10}}:-3.2\left(c=0.5, \mathrm{CHCl}_{3}\right) .89 \%$ ee, HPLC analysis of the product: Daicel CHIRALCEL OD-H column; $10 \% \mathrm{i}-\mathrm{PrOH}$ in hexanes; $1.0 \mathrm{~mL} / \mathrm{min} ; 35^{\circ} \mathrm{C}$; retention times: $11.3 \mathrm{~min}$ (minor), $16.4 \mathrm{~min}$ (major).

${ }^{1} \mathrm{H}$ NMR $\left(400 \mathrm{MHz}^{\mathrm{CDCl}} 3\right) \delta 7.45-7.32(\mathrm{~m}, 5 \mathrm{H}), 6.97$ (d, J = 8.4 Hz, 1H), 6.89 (d, J= $2.4 \mathrm{~Hz}, 1 \mathrm{H}), 6.78\left(\mathrm{dd}, J_{1}=8.8 \mathrm{~Hz}, J_{2}=2.8 \mathrm{~Hz}, 1 \mathrm{H}\right), 5.01(\mathrm{~s}, 2 \mathrm{H}), 4.03-3.89(\mathrm{~m}, 2 \mathrm{H})$, $3.85(\mathrm{~d}, J=15.2 \mathrm{~Hz}, 1 \mathrm{H}), 3.78-3.68(\mathrm{~m}, 2 \mathrm{H}), 2.41(\mathrm{br} \mathrm{s}, 1 \mathrm{H}), 1.64(\mathrm{~s}, 3 \mathrm{H}), 1.61(\mathrm{~s}, 3 \mathrm{H})$ ppm.

${ }^{13} \mathrm{C}$ NMR $\left(100 \mathrm{MHz}, \mathrm{CDCl}_{3}\right) \delta 155.0,149.7,140.1,136.9,128.6,128.1,127.6,122.9$, $114.2,113.1,82.0,78.2,70.5,64.8,62.7,28.8,26.9$ ppm.

HRMS (CI) Calcd for $\mathrm{C}_{19} \mathrm{H}_{22} \mathrm{NaO}_{4}[\mathrm{M}+\mathrm{Na}]^{+}$: 337.1416, Found: 337.1478.<smiles>C=CCOc1ccc2c(c1)C(C)(C)OC[C@@H](CO)O2</smiles>

2j 


\section{(R)-(7-(allyloxy)-5,5-dimethyl-3,5-dihydro-2H-benzo[e][1,4]dioxepin-2-}

yl)methanol (2j) was prepared from 2-(5-(allyloxy)-2-(oxetan-3yloxy)phenyl)propan-2-ol $\mathbf{1 j}(79.3 \mathrm{mg}, 0.30 \mathrm{mmol})$ according to the general procedure (rt, $20 \mathrm{~h}$; purification by flash column chromatography: petroleum ether/ethyl acetate $=10: 3,75.1 \mathrm{mg}$, colorless oil, 95\% yield).

$[\alpha]_{D^{10}}:-4.0\left(c=0.5, \mathrm{CHCl}_{3}\right) . \quad 87 \%$ ee, HPLC analysis of the product: Daicel CHIRALCEL OD-H column; $10 \% \mathrm{i}-\mathrm{PrOH}$ in hexanes; $1.0 \mathrm{~mL} / \mathrm{min} ; 35{ }^{\circ} \mathrm{C}$; retention times: 7.3 min (minor), 10.4 min (major).

${ }^{1} \mathrm{H}$ NMR $\left(400 \mathrm{MHz}, \mathrm{CDCl}_{3}\right) \delta 6.93(\mathrm{~d}, J=8.4 \mathrm{~Hz}, 1 \mathrm{H}), 6.82(\mathrm{~d}, J=2.4 \mathrm{~Hz}, 1 \mathrm{H}), 6.70$ $\left(\mathrm{dd}, J_{1}=8.4 \mathrm{~Hz}, J_{2}=2.4 \mathrm{~Hz}, 1 \mathrm{H}\right), 6.08-5.99(\mathrm{~m}, 1 \mathrm{H}), 5.40(\mathrm{~d}, J=17.6 \mathrm{~Hz}, 1 \mathrm{H}), 5.28$ (t, $J=2.0 \mathrm{~Hz}, 1 \mathrm{H}), 4.48(\mathrm{~d}, J=5.2 \mathrm{~Hz}, 2 \mathrm{H}), 4.01-3.87(\mathrm{~m}, 2 \mathrm{H}), 3.82(\mathrm{~d}, J=12.8 \mathrm{~Hz}, 1 \mathrm{H})$, 3.76-3.67 (m, 2H), 2.42 (br s, 1H), $1.62(\mathrm{~s}, 3 \mathrm{H}), 1.59$ (s, 3H) ppm.

${ }^{13} \mathrm{C}$ NMR $\left(100 \mathrm{MHz}_{2} \mathrm{CDCl}_{3}\right) \delta 154.8,149.6,140.0,133.3,122.8,117.8,114.0,113.0$, $82.0,78.2,69.3,64.8,62.7,28.7,26.9 \mathrm{ppm}$.

HRMS (CI) Calcd for $\mathrm{C}_{15} \mathrm{H}_{20} \mathrm{NaO}_{4}[\mathrm{M}+\mathrm{Na}]^{+}: 287.1259$ Found: 287.1332.<smiles>C#CCOc1ccc2c(c1)C(C)(C)OC[C@@H](CO)O2</smiles>

2k

(R)-(5,5-Dimethyl-7-(prop-2-yn-1-yloxy)-3,5-dihydro-2H-benzo[e][1,4]dioxepin2-yl)methanol (2k) was prepared from 2-(2-(oxetan-3-yloxy)-5-(prop- 2-yn-1yloxy)phenyl)propan-2-ol $\mathbf{1 k}(78.7 \mathrm{mg}, 0.30 \mathrm{mmol})$ according to the general procedure (rt, $36 \mathrm{~h}$; purification by flash column chromatography: petroleum ether/ethyl acetate $=10: 3,73.6 \mathrm{mg}$, colorless oil, $94 \%$ yield).

$[\alpha]_{D^{10}}:-2.9\left(c=0.5, \mathrm{CHCl}_{3}\right) .88 \%$ ee, HPLC analysis of the product: Daicel CHIRALCEL OD-H column; $10 \% \mathrm{i}-\mathrm{PrOH}$ in hexanes; $1.0 \mathrm{~mL} / \mathrm{min} ; 35{ }^{\circ} \mathrm{C}$; retention times: $11.4 \mathrm{~min}$ (minor), $17.8 \mathrm{~min}$ (major). 
${ }^{1} \mathrm{H}$ NMR $\left(400 \mathrm{MHz}, \mathrm{CDCl}_{3}\right) \delta 6.95(\mathrm{~d}, J=7.6 \mathrm{~Hz}, 1 \mathrm{H}), 6.86(\mathrm{~d}, J=2.0 \mathrm{~Hz}, 1 \mathrm{H}), 6.76(\mathrm{t}$, $J=2.4 \mathrm{~Hz}, 1 \mathrm{H}), 4.63(\mathrm{~s}, 2 \mathrm{H}), 4.0-3.86(\mathrm{~m}, 2 \mathrm{H}), 3.82(\mathrm{~d}, J=13.2 \mathrm{~Hz}, 1 \mathrm{H}), 3.75-3.65(\mathrm{~m}$, 2H), $2.53(\mathrm{~s}, 2 \mathrm{H}), 1.62(\mathrm{~s}, 3 \mathrm{H}), 1.58(\mathrm{~s}, 3 \mathrm{H}) \mathrm{ppm}$.

${ }^{13} \mathrm{C}$ NMR $\left(100 \mathrm{MHz}, \mathrm{CDCl}_{3}\right) \delta 153.4,150.2,140.1,122.9,114.3,113.3,82.1,78.6,78.1$, $75.7,64.8,62.6,56.2,28.7,26.8$ ppm.

HRMS (CI) Calcd for $\mathrm{C}_{15} \mathrm{H}_{18} \mathrm{NaO}_{4}[\mathrm{M}+\mathrm{Na}]^{+}:$285.1103, Found: 285.1193.<smiles>CCC1(CC)OC[C@@H](CO)Oc2ccccc21</smiles>

(R)-(5,5-Diethyl-3,5-dihydro-2H-benzo[e][1,4]dioxepin-2-yl)methanol (21) was prepared from 3-(2-(oxetan-3-yloxy)phenyl)pentan-3-ol 11 (70.9 mg, $0.30 \mathrm{mmol}$ ) according to the general procedure ( $\mathrm{rt}, 48 \mathrm{~h}$; purification by flash column chromatography: petroleum ether/ethyl acetate $=10: 3,67.0 \mathrm{mg}$, colorless oil, $94 \%$ yield).

$[\alpha]_{\mathrm{D}^{10}}:-9.1\left(c=0.5, \mathrm{CHCl}_{3}\right) .93 \%$ ee, HPLC analysis of the product: Daicel CHIRALCEL OD-H column; $5 \% i-\mathrm{PrOH}$ in hexanes; $1.0 \mathrm{~mL} / \mathrm{min} ; 35^{\circ} \mathrm{C}$; retention times: $11.0 \mathrm{~min}$ (minor), $12.5 \mathrm{~min}$ (major).

${ }^{1} \mathbf{H}$ NMR $\left(400 \mathrm{MHz}, \mathrm{CDCl}_{3}\right) \delta 7.20(\mathrm{t}, J=6.4 \mathrm{~Hz}, 1 \mathrm{H}), 7.12-7.02(\mathrm{~m}, 3 \mathrm{H}), 4.04-3.99$ $(\mathrm{m}, 1 \mathrm{H}), 3.85-3.81(\mathrm{~m}, 3 \mathrm{H}), 3.73\left(\mathrm{dd}, J_{1}=11.6 \mathrm{~Hz}, J_{2}=4.0 \mathrm{~Hz}, 1 \mathrm{H}\right), 2.49$ (br s, $\left.1 \mathrm{H}\right)$, 2.11-1.89 (m, 3H), 1.82-1.73 (m, 1H), $0.95(\mathrm{t}, J=7.2 \mathrm{~Hz}, 3 \mathrm{H}), 0.75(\mathrm{t}, J=7.6 \mathrm{~Hz}, 3 \mathrm{H})$ ppm.

${ }^{13} \mathrm{C}$ NMR $\left(100 \mathrm{MHz} \mathrm{CDCl}_{3}\right) \delta 154.8,136.2,128.5,128.4,123.9,122.9,84.2,81.0,63.2$, 63.1, 30.8, 30.3, 8.3, 7.9 ppm.

HRMS (CI) Calcd for $\mathrm{C}_{14} \mathrm{H}_{20} \mathrm{NaO}_{3}[\mathrm{M}+\mathrm{Na}]^{+}:$259.1310, Found: 259.1419. 


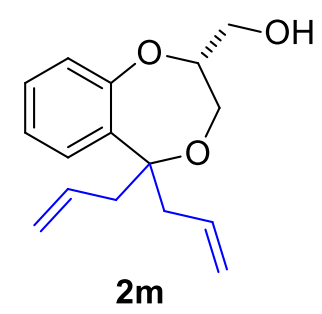

(R)-(5,5-Diallyl-3,5-dihydro-2H-benzo[e][1,4]dioxepin-2-yl)methanol (2m) was prepared from 4-(2-(oxetan-3-yloxy)phenyl)hepta-1,6-dien-4-ol 1m (78.1 mg, 0.30 mmol) according to the general procedure $\left(50{ }^{\circ} \mathrm{C}\right.$ in an oil bath, $60 \mathrm{~h}$; purification by flash column chromatography: petroleum ether/ethyl acetate $=10: 3,69.1 \mathrm{mg}$, colorless oil, $88 \%$ yield).

$[\alpha]_{\mathrm{D}^{10}}:-26.2\left(c=0.5, \mathrm{CHCl}_{3}\right) .95 \%$ ee, HPLC analysis of the product: Daicel CHIRALCEL OD-H column; $2 \%$ i-PrOH in hexanes; $1.0 \mathrm{~mL} / \mathrm{min} ; 35^{\circ} \mathrm{C}$; retention times: $19.5 \mathrm{~min}$ (major), $20.9 \mathrm{~min}$ (minor).

${ }^{1} \mathrm{H}$ NMR $\left(400 \mathrm{MHz}, \mathrm{CDCl}_{3}\right) \delta 7.22(\mathrm{t}, J=7.6 \mathrm{~Hz}, 1 \mathrm{H}), 7.14(\mathrm{~d}, J=7.2 \mathrm{~Hz}, 1 \mathrm{H}), 7.09(\mathrm{~d}$, $J=7.6 \mathrm{~Hz}, 1 \mathrm{H}), 7.04(\mathrm{~d}, J=8.0 \mathrm{~Hz}, 1 \mathrm{H}), 5.95-5.84(\mathrm{~m}, 1 \mathrm{H}), 5.65-5.54(\mathrm{~m}, 1 \mathrm{H}), 5.11(\mathrm{t}$, $J=15.2 \mathrm{~Hz}, 2 \mathrm{H}), 4.98(\mathrm{t}, J=9.6 \mathrm{~Hz}, 2 \mathrm{H}), 4.03-3.98(\mathrm{~m}, 1 \mathrm{H}), 3.92-3.88(\mathrm{~m}, 3 \mathrm{H}), 3.74$ $\left(\mathrm{dd}, J_{1}=11.6 \mathrm{~Hz}, J_{2}=4.0 \mathrm{~Hz}, 1 \mathrm{H}\right), 2.84-2.68(\mathrm{~m}, 3 \mathrm{H}), 2.61-2.56(\mathrm{~m}, 1 \mathrm{H}), 2.50(\mathrm{br} \mathrm{s}, 1 \mathrm{H})$ ppm.

${ }^{13} \mathrm{C}$ NMR (100 MHz, $\left.\mathrm{CDCl}_{3}\right) \delta 154.7,135.5,133.8,133.5,128.9,128.3,124.0,123.0$, $118.2,82.7,80.5,63.8,63.1,43.2,42.8 \mathrm{ppm}$.

HRMS (CI) Calcd for $\mathrm{C}_{16} \mathrm{H}_{20} \mathrm{NaO}_{3}[\mathrm{M}+\mathrm{Na}]^{+}:$283.1310, Found: 283.1388 .

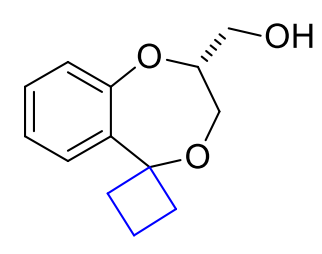

2n

(R)-(2,3-Dihydrospiro[benzo[ $e][1,4]$ dioxepine-5,1'-cyclobutan]-2-yl)methanol

(2n) was prepared from 1-(2-(oxetan-3-yloxy)phenyl)cyclobutanol 1n $(66.1 \mathrm{mg}$, $0.30 \mathrm{mmol}$ ) according to the general procedure ( $\mathrm{rt}, 36 \mathrm{~h}$; purification by flash 
column chromatography: petroleum ether/ethyl acetate $=10: 3,65.0 \mathrm{mg}$, white solid, $98 \%$ yield).

$[\alpha]_{\mathrm{D}^{10}}:-24.7\left(c=0.5, \mathrm{CHCl}_{3}\right) .98 \%$ ee, HPLC analysis of the product: Daicel CHIRALCEL OD-H column; $10 \% i$-PrOH in hexanes; $1.0 \mathrm{~mL} / \mathrm{min} ; 35^{\circ} \mathrm{C}$; retention times: $12.4 \mathrm{~min}$ (major), $13.4 \mathrm{~min}$ (minor).

${ }^{1} \mathrm{H}$ NMR $\left(400 \mathrm{MHz}, \mathrm{CDCl}_{3}\right) \delta 7.42(\mathrm{~d}, J=7.6 \mathrm{~Hz}, 1 \mathrm{H}), 7.23(\mathrm{t}, J=7.6 \mathrm{~Hz}, 1 \mathrm{H}), 7.11(\mathrm{t}$, $J=7.6 \mathrm{~Hz}, 1 \mathrm{H}), 7.05(\mathrm{~d}, J=8.0 \mathrm{~Hz}, 1 \mathrm{H}), 4.03-3.97(\mathrm{~m}, 1 \mathrm{H}), 3.94-3.84(\mathrm{~m}, 2 \mathrm{H}), 3.70(\mathrm{~d}$, $J=5.2 \mathrm{~Hz}, 2 \mathrm{H}), 2.73-2.70(\mathrm{~m}, 1 \mathrm{H}), 2.58-2.51(\mathrm{~m}, 1 \mathrm{H}), 2.37-2.28(\mathrm{~m}, 2 \mathrm{H}), 2.20-2.15$ (m, 1H), 1.85-1.78 (m, 1H), 1.61-1.50 (m, 1H) ppm.

${ }^{13} \mathrm{C}$ NMR (100 MHz, $\left.\mathrm{CDCl}_{3}\right) \delta 157.4,135.9,128.9,126.1,123.7,121.9,82.3,81.0,65.6$, 62.6, 32.1, 32.9, $12.9 \mathrm{ppm}$.

HRMS (CI) Calcd for $\mathrm{C}_{13} \mathrm{H}_{16} \mathrm{NaO}_{3}[\mathrm{M}+\mathrm{Na}]^{+}:$243.0997, Found: 243.1072 .

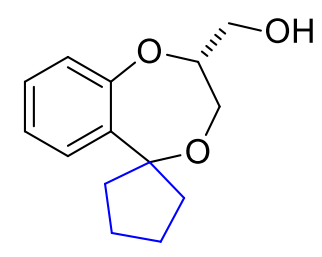

20

\section{(R)-(2,3-Dihydrospiro[benzo[e][1,4]dioxepine-5,1'-cyclopentan]-2-yl)methanol}

(2o) was prepared from 1-(2-(oxetan-3-yloxy)phenyl)cyclopentanol 1o (70.3 mg, $0.30 \mathrm{mmol}$ ) according to the general procedure $(\mathrm{rt}, 48 \mathrm{~h}$; purification by flash column chromatography: petroleum ether/ethyl acetate $=10: 3,59.8 \mathrm{mg}$, white solid, $85 \%$ yield).

$[\alpha]_{\mathrm{D}^{10}}:-21.5\left(c=0.5, \mathrm{CHCl}_{3}\right) .92 \%$ ee, HPLC analysis of the product: Daicel CHIRALCEL OD-H column; $5 \%$ i-PrOH in hexanes; $1.0 \mathrm{~mL} / \mathrm{min} ; 35^{\circ} \mathrm{C}$; retention times: $15.6 \mathrm{~min}$ (minor), $23.1 \mathrm{~min}$ (major).

${ }^{1} \mathrm{H}$ NMR $\left(400 \mathrm{MHz},\left(\mathrm{CD}_{3}\right)_{2} \mathrm{CO}\right) \delta 7.19(\mathrm{~d}, J=7.6 \mathrm{~Hz}, 1 \mathrm{H}), 7.07(\mathrm{t}, J=7.6 \mathrm{~Hz}, 1 \mathrm{H}), 6.92$ $(\mathrm{t}, J=9.2 \mathrm{~Hz}, 2 \mathrm{H}), 3.90(\mathrm{t}, J=6.4 \mathrm{~Hz}, 1 \mathrm{H}), 3.81-3.74(\mathrm{~m}, 3 \mathrm{H}), 3.66-3.61(\mathrm{~m}, 1 \mathrm{H}), 3.54-$ $3.49(\mathrm{~m}, 1 \mathrm{H}), 2.42-2.39(\mathrm{~m}, 1 \mathrm{H}), 2.10-1.95(\mathrm{~m}, 2 \mathrm{H}), 1.77-1.57$ (m, 5H) ppm. 
${ }^{13} \mathrm{C}$ NMR $\left.\left(100 \mathrm{MHz},\left(\mathrm{CD}_{3}\right)_{2} \mathrm{CO}\right)\right) \delta 157.8,137.3,128.2,126.3,123.3,121.2,87.6,82.7$, 65.0, 61.9, 37.0, 35.5, 22.2, $22.0 \mathrm{ppm}$.

HRMS (CI) Calcd for $\mathrm{C}_{14} \mathrm{H}_{18} \mathrm{NaO}_{3}[\mathrm{M}+\mathrm{Na}]^{+}:$257.1154, Found: 257.1250

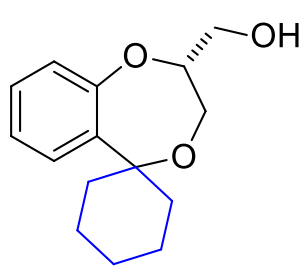

$2 p$

\section{(R)-(2,3-Dihydrospiro[benzo[e][1,4]dioxepine-5,1'-cyclohexan]-2-yl)methanol}

(2p) was prepared from 1-(2-(oxetan-3-yloxy)phenyl)cyclohexanol 1p (74.5 mg, $0.30 \mathrm{mmol})$ according to the general procedure $\left(50{ }^{\circ} \mathrm{C}\right.$ in an oil bath, $24 \mathrm{~h}$; purification by flash column chromatography: petroleum ether/ethyl acetate $=10: 3$, $67.8 \mathrm{mg}$, white solid, 91\% yield).

$[\alpha]_{D^{10}}:-26.3\left(c=0.5, \mathrm{CHCl}_{3}\right) .93 \%$ ee, HPLC analysis of the product: Daicel CHIRALCEL OD-H column; $10 \%$-PrOH in hexanes; $1.0 \mathrm{~mL} / \mathrm{min} ; 35^{\circ} \mathrm{C}$; retention times: $12.1 \mathrm{~min}$ (minor), $19.7 \mathrm{~min}$ (major).

${ }^{1}$ H NMR $\left(400 \mathrm{MHz}, \mathrm{CDCl}_{3}\right) \delta 7.27(\mathrm{~d}, J=7.2 \mathrm{~Hz}, 1 \mathrm{H}), 7.17(\mathrm{t}, J=7.6 \mathrm{~Hz}, 1 \mathrm{H}), 7.07-$ $7.01(\mathrm{~m}, 2 \mathrm{H}), 4.01-3.85(\mathrm{~m}, 3 \mathrm{H}), 3.78-3.71(\mathrm{~m}, 2 \mathrm{H}), 2.60$ (br s, 1H), $2.54(\mathrm{~d}, J=14.4$ $\mathrm{Hz}, 1 \mathrm{H}), 2.11-2.05$ (m, 1H), 1.96-1.56 (m, 6H), 1.47-1,39 (m, 1H), 1.29-1.20 (m, 1H) ppm.

${ }^{13} \mathrm{C}$ NMR (100 MHz, $\left.\mathrm{CDCl}_{3}\right) \delta 156.3,139.5,128.3,126.5,124.0,122.3,82.1,78.3,64.1$, 62.8, 35.7, 34.0, 25.6, 21.9, $21.7 \mathrm{ppm}$.

HRMS (CI) Calcd for $\mathrm{C}_{15} \mathrm{H}_{20} \mathrm{NaO}_{3}[\mathrm{M}+\mathrm{Na}]^{+}:$271.1310, Found: 271.1403.

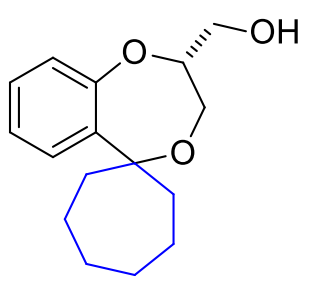

$2 q$ 


\section{(R)-(2,3-Dihydrospiro[benzo[e][1,4]dioxepine-5,1'-cycloheptan]-2-yl)methanol}

(2q) was prepared from 1-(2-(oxetan-3-yloxy)phenyl)cycloheptan-1-ol 1q (78.7 mg, $0.30 \mathrm{mmol}$ ) according to the general procedure $\left(50{ }^{\circ} \mathrm{C}\right.$ in an oil bath, $48 \mathrm{~h}$; purification by flash column chromatography: petroleum ether/ethyl acetate $=2: 1$, $78.0 \mathrm{mg}$, white solid, 99\% yield)

$[\alpha]_{\mathrm{D}^{22}}:-19.2\left(c=0.5, \mathrm{CHCl}_{3}\right) .94 \%$ ee, HPLC analysis of the product: Daicel CHIRALCEL OD-H column; $10 \% \mathrm{i}-\mathrm{PrOH}$ in hexanes; $1.0 \mathrm{~mL} / \mathrm{min} ; 35{ }^{\circ} \mathrm{C}$; retention times: 8.1 min (minor), 14.8 min (major).

${ }^{1} \mathrm{H}$ NMR $\left(400 \mathrm{MHz}, \mathrm{CDCl}_{3}\right) \delta 7.25(\mathrm{~d}, J=7.2 \mathrm{~Hz}, 1 \mathrm{H}), 7.18\left(\mathrm{td}, J_{1}=7.6 \mathrm{~Hz}, J_{2}=1.6 \mathrm{~Hz}\right.$, 1H), $7.07(\mathrm{t}, J=7.6 \mathrm{~Hz}, 1 \mathrm{H}), 7.01(\mathrm{~d}, J=8.0 \mathrm{~Hz}, 1 \mathrm{H}), 4.02-3.97(\mathrm{~m}, 1 \mathrm{H}), 3.39-3.82(\mathrm{~m}$, 2H), 3.80-3.72 (m, 2H), 2.49-2.43 (m, 1H), 2.26-2.20 (m, 1H), 2.14-2.08 (m, 1H), 1.89$1.47(\mathrm{~m}, 10 \mathrm{H}) \mathrm{ppm}$.

${ }^{13} \mathrm{C}$ NMR $\left(100 \mathrm{MHz}, \mathrm{CDCl}_{3}\right) \delta 154.9,140.2,128.4,127.0,124.1,122.8,83.7,81.6,63.6$, $63.1,39.7,38.5,29.8,29.5,22.5,22.0$ ppm.

HRMS (CI) Calcd for $\mathrm{C}_{16} \mathrm{H}_{22} \mathrm{NaO}_{3}[\mathrm{M}+\mathrm{Na}]^{+}:$285.1467, Found: 285.1482.

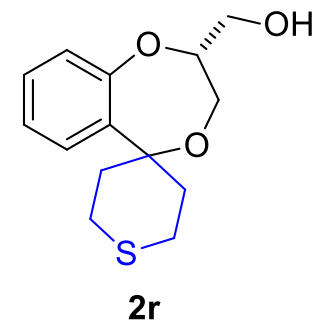

(R)-(2,2',3,3',5',6'-Hexahydrospiro[benzo[e][1,4]dioxepine-5,4'-thiopyran]-2-

yl)methanol (2r) was prepared from 4-(2-(oxetan-3-yloxy)phenyl)tetrahydro-2Hthiopyran-4-ol $1 \mathbf{r}(79.9 \mathrm{mg}, 0.30 \mathrm{mmol})$ according to the general procedure $\left(50{ }^{\circ} \mathrm{C}\right.$ in an oil bath, $48 \mathrm{~h}$; purification by flash column chromatography: petroleum ether/ethyl acetate $=2: 1,78.8 \mathrm{mg}$, white solid, 99\% yield) $[\alpha]_{\mathrm{D}^{22}}:-53.4\left(c=0.5, \mathrm{CHCl}_{3}\right) .90 \%$ ee, HPLC analysis of the product: Daicel CHIRALPAK AS-H column; 3\% $i$-PrOH in hexanes; $1.0 \mathrm{~mL} / \mathrm{min} ; 35^{\circ} \mathrm{C}$; retention times: $27.1 \mathrm{~min}$ (major), $29.5 \mathrm{~min}$ (minor). 
${ }^{1} \mathbf{H}$ NMR $\left(400 \mathrm{MHz} \mathrm{CDCl}_{3}\right) \delta 7.20(\mathrm{t}, J=8.4 \mathrm{~Hz}, 2 \mathrm{H}), 7.08(\mathrm{t}, J=7.6 \mathrm{~Hz}, 1 \mathrm{H}), 7.01(\mathrm{~d}$, $J=8.0 \mathrm{~Hz}, 1 \mathrm{H}), 4.00-3.90(\mathrm{~m}, 1 \mathrm{H}), 3.90-3.70(\mathrm{~m}, 4 \mathrm{H}), 3.24(\mathrm{t}, J=7.6 \mathrm{~Hz}, 1 \mathrm{H}), 3.10(\mathrm{t}$, $J=12.4 \mathrm{~Hz}, 1 \mathrm{H}), 2.68(\mathrm{~d}, J=14.4 \mathrm{~Hz}, 1 \mathrm{H}), 2.55$ (br s, 1H), 2.45 (d, J = $13.2 \mathrm{~Hz}, 1 \mathrm{H})$, $2.35(\mathrm{~d}, J=16.8 \mathrm{~Hz}, 2 \mathrm{H}), 2.29-2.23(\mathrm{~m}, 1 \mathrm{H}), 1.81\left(\mathrm{td}, J_{1}=14.0 \mathrm{~Hz}, J_{2}=3.2 \mathrm{~Hz}, 1 \mathrm{H}\right)$ ppm.

${ }^{13} \mathrm{C}$ NMR $\left(100 \mathrm{MHz}, \mathrm{CDCl}_{3}\right) \delta 155.0,138.4,128.9,126.7,124.5,122.8,81.5,76.8,63.3$, $62.9,36.7,36.1,23.9,23.8 \mathrm{ppm}$.

HRMS (CI) Calcd for $\mathrm{C}_{14} \mathrm{H}_{18} \mathrm{NaO}_{3} \mathrm{~S}[\mathrm{M}+\mathrm{Na}]^{+}$: 289.0874, Found: 289.0892.<smiles>OC[C@H]1COC2(CCOCC2)c2ccccc2O1</smiles>

2s

(R)-(2,2',3,3',5',6'-Hexahydrospiro[benzo[e][1,4]dioxepine-5,4'-pyran]-2-

yl)methanol (2s) was prepared from 4-(2-(oxetan-3-yloxy)phenyl)tetrahydro-2Hpyran-4-ol $1 \mathrm{~s}(75.1 \mathrm{mg}, 0.30 \mathrm{mmol})$ according to the general procedure $\left(50{ }^{\circ} \mathrm{C}\right.$ in an oil bath, 48 h; purification by flash column chromatography: petroleum ether/ethyl acetate $=2: 1,74.0 \mathrm{mg}$, white solid, 99\% yield).

$[\alpha]_{D^{22}}:-26.1\left(c=0.5, \mathrm{CHCl}_{3}\right) .93 \%$ ee, HPLC analysis of the product: Daicel CHIRALCEL OD-H column; $10 \%$-PrOH in hexanes; $1.0 \mathrm{~mL} / \mathrm{min} ; 35^{\circ} \mathrm{C}$; retention times: $14.0 \mathrm{~min}$ (minor), $20.2 \mathrm{~min}$ (major).

${ }^{1}$ H NMR $\left(400 \mathrm{MHz}, \mathrm{CDCl}_{3}\right) \delta 7.26-7.19(\mathrm{~m}, 2 \mathrm{H}), 7.09(\mathrm{t}, J=7.6 \mathrm{~Hz}, 1 \mathrm{H}), 7.03(\mathrm{~d}, J=$ $8.0 \mathrm{~Hz}, 1 \mathrm{H}), 4.01-3.87(\mathrm{~m}, 5 \mathrm{H}), 3.78-3.75(\mathrm{~m}, 4 \mathrm{H}), 2.58$ (br s, $1 \mathrm{H}), 2.49$ (dd, $J_{1}=14.4$ $\left.\mathrm{Hz}, J_{2}=1.6 \mathrm{~Hz}, 1 \mathrm{H}\right), 2.31\left(\mathrm{td}, J_{1}=13.2 \mathrm{~Hz}, J_{2}=4.8 \mathrm{~Hz}, 1 \mathrm{H}\right), 1.95\left(\mathrm{dd}, J_{1}=13.6 \mathrm{~Hz}, J_{2}=\right.$ $2.4 \mathrm{~Hz}, 1 \mathrm{H}), 1.87-1.79(\mathrm{~m}, 1 \mathrm{H}) \mathrm{ppm}$.

${ }^{13} \mathrm{C}$ NMR $\left(100 \mathrm{MHz}, \mathrm{CDCl}_{3}\right) \delta 156.3,137.9,129.0,126.3,124.4,122.6,82.0,76.0,64.2$, $63.9,63.5,62.8,35.9,34.3 \mathrm{ppm}$.

HRMS (CI) Calcd for $\mathrm{C}_{14} \mathrm{H}_{18} \mathrm{NaO}_{4}[\mathrm{M}+\mathrm{Na}]^{+}:$273.1103, Found: 273.1124 . 
<smiles>CC1(C)OC[C@@H](CO)Sc2ccccc21</smiles>

2b-S

(R)-(5,5-Dimethyl-3,5-dihydro-2H-benzo[e][1,4]oxathiepin-2-yl)methanol (2b-S) was prepared from 2-(2-(oxetan-3-ylthio)phenyl)propan-2-ol 1b-S (67.3 mg, 0.30 $\mathrm{mmol}$ ) according to the general procedure (rt, $12 \mathrm{~h}$; purification by flash column chromatography: petroleum ether/ethyl acetate $=4: 1,65.6 \mathrm{mg}$, colorless oil, $97 \%$ yield).

$[\alpha]_{\mathrm{D}^{10}}:+39.6\left(c=0.5, \mathrm{CHCl}_{3}\right) .90 \%$ ee, HPLC analysis of the product: Daicel CHIRALCEL OD-H column; 5\% $i-\mathrm{PrOH}$ in hexanes; $1.0 \mathrm{~mL} / \mathrm{min} ; 35^{\circ} \mathrm{C}$; retention times: $11.9 \mathrm{~min}$ (minor), $13.4 \mathrm{~min}$ (major).

${ }^{1} \mathrm{H}$ NMR $\left(400 \mathrm{MHz}, \mathrm{CDCl}_{3}\right) \delta 7.47(\mathrm{~d}, J=7.6 \mathrm{~Hz}, 1 \mathrm{H}), 7.26(\mathrm{~d}, J=4.0 \mathrm{~Hz}, 2 \mathrm{H}), 7.16-$ $7.13(\mathrm{~m}, 1 \mathrm{H}), 4.02\left(\mathrm{dd}, J_{1}=12.8 \mathrm{~Hz}, J_{2}=5.6 \mathrm{~Hz}, 1 \mathrm{H}\right), 3.61-3.47(\mathrm{~m}, 3 \mathrm{H}), 3.32-3.26(\mathrm{~m}$, 1H), 2.54 (br s, 1H), 1.65 (s, 6H) ppm.

${ }^{13} \mathrm{C}$ NMR $\left(100 \mathrm{MHz}, \mathrm{CDCl}_{3}\right) \delta 148.3,136.3,128.8,127.7,127.4,81.8,63.5,62.7,50.5$, 29.0, 28.9 ppm.

HRMS (CI) Calcd for $\mathrm{C}_{12} \mathrm{H}_{16} \mathrm{NaO}_{2} \mathrm{~S}[\mathrm{M}+\mathrm{Na}]^{+}$: 247.0769, Found: 247.0837.

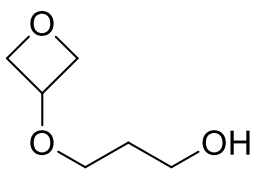

3

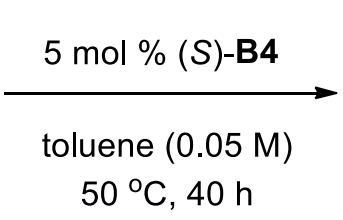

$50{ }^{\circ} \mathrm{C}, 40 \mathrm{~h}$<smiles>OC[C@H]1COCCCO1</smiles>

4

(R)-(1,4-Dioxepan-2-yl)methanol (4) was prepared from 3-(oxetan-3yloxy)propan-1-ol $3(39.7 \mathrm{mg}, 0.30 \mathrm{mmol})$ according to the general procedure (50 
${ }^{\circ} \mathrm{C}$ in an oil bath; $40 \mathrm{~h}$ purification by flash column chromatography: petroleum ether/ethyl acetate = 2:1, $28.3 \mathrm{mg}$, colorless oil, 71\% yield).

$[\alpha]_{\mathrm{D}^{24}}:-8.9\left(c=1.0, \mathrm{CHCl}_{3}\right) .69 \%$ ee, HPLC analysis of the O-4-nitrobenzoyl derivative: Daicel CHIRALCEL OD-H column; $2 \% i-\mathrm{PrOH}$ in hexanes; $1.0 \mathrm{~mL} / \mathrm{min}$; $35^{\circ} \mathrm{C}$; retention times: $43.1 \mathrm{~min}$ (minor), $45.2 \mathrm{~min}$ (major).

${ }^{1} \mathrm{H}$ NMR $\left(400 \mathrm{MHz}, \mathrm{CDCl}_{3}\right) \delta$ 4.04-3.99 (m, 1H), 3.87-3.80 (m, 2H), 3.74-3.67 (m, 3H), 3.57-3.41 (m, 3H), 2.57 (br s, 1H), 2.03-1.90 (m, 2H) ppm.

${ }^{13} \mathrm{C}$ NMR (100 MHz, $\left.\mathrm{CDCl}_{3}\right) \delta 81.6,73.0,69.3,67.9,62.9,32.6 \mathrm{ppm}$.

HRMS (CI) Calcd for $\mathrm{C}_{6} \mathrm{H}_{12} \mathrm{NaO}_{3}[\mathrm{M}+\mathrm{Na}]^{+}:$155.0684, Found: 155.0696.

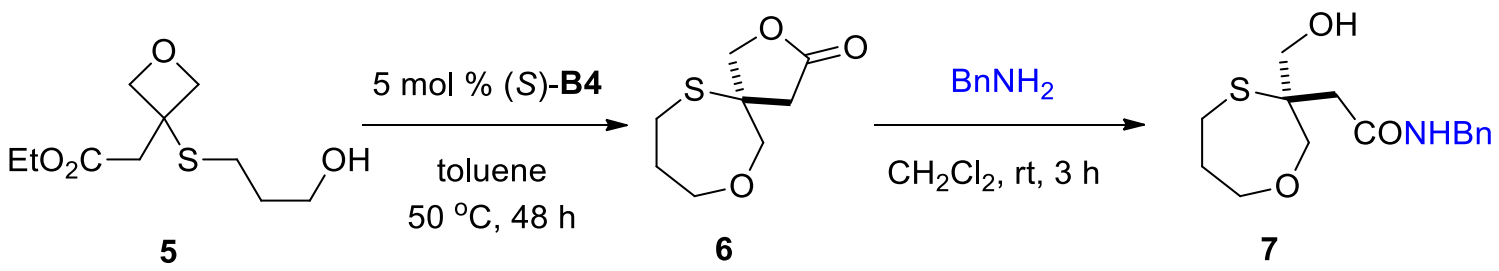

(R)-2,10-Dioxa-6-thiaspiro[4.6]undecan-3-one (6) was prepared from ethyl 2-(3((3-hydroxypropyl)thio)oxetan-3-yl)acetate 5 (70.3 $\mathrm{mg}, 0.30 \mathrm{mmol}$ ) according to the general procedure $\left(50{ }^{\circ} \mathrm{C}\right.$ in an oil bath, $48 \mathrm{~h}$; purification by flash column chromatography: petroleum ether/ethyl acetate $=2: 1,31.6 \mathrm{mg}$, colorless liquid, $56 \%$ yield).

$[\alpha]_{\mathrm{D}^{22}}:+16.2\left(c=0.5, \mathrm{CHCl}_{3}\right)$.

${ }^{1} \mathrm{H}$ NMR $\left(400 \mathrm{MHz}, \mathrm{CDCl}_{3}\right) \delta 4.40(\mathrm{~d}, J=6.4 \mathrm{~Hz}, 1 \mathrm{H}), 4.28(\mathrm{~d}, J=9.6 \mathrm{~Hz}, 1 \mathrm{H}), 3.99-$ $3.86(\mathrm{~m}, 3 \mathrm{H}), 3.76(\mathrm{~d}, J=12.8 \mathrm{~Hz}, 1 \mathrm{H}), 2.88-2.73(\mathrm{~m}, 2 \mathrm{H}), 2.62(\mathrm{~s}, 2 \mathrm{H}) 2.18-2.09(\mathrm{~m}$, 2H) ppm.

${ }^{13} \mathrm{C}$ NMR (100 MHz, $\left.\mathrm{CDCl}_{3}\right) \delta 174.6,79.9,75.5,71.7,51.6,39.2,32.8,26.9 \mathrm{ppm}$.

HRMS (CI) Calcd for $\mathrm{C}_{8} \mathrm{H}_{12} \mathrm{NaNO}_{3}$ [M + Na] $]^{+}$211.0405, Found: 211.0346. 
<smiles>O=C(CC[C@@]1(CO)COCCCS1)NCc1ccccc1</smiles>

7

(S)-N-Benzyl-2-(3-(hydroxymethyl)-1,4-oxathiepan-3-yl)acetamide (7). A mixture of $6(38.0 \mathrm{mg}, 0.20 \mathrm{mmol})$ and benzylamine $(0.4 \mathrm{~mL})$ was stirred at room temperature for $3 \mathrm{~h}$. The mixture was diluted with $\mathrm{CH}_{2} \mathrm{Cl}_{2}(40 \mathrm{~mL})$ and then washed with $\mathrm{HCl}$ aqueous solution $(2 \times 20 \mathrm{~mL}, 1 \mathrm{M})$. The organic layer was dried over anhydrous $\mathrm{Na}_{2} \mathrm{SO}_{4}$, and concentrated under reduced pressure to give the desired product 7 (purification by flash column chromatography: petroleum ether/ethyl acetate $=2: 1,59.0 \mathrm{mg}$, white solid, 99\% yield).

$[\alpha]_{D^{22}}:-8.4\left(c=0.5, \mathrm{CHCl}_{3}\right) .91 \%$ ee, HPLC analysis of the product: Daicel CHIRALCEL OD-H column; $10 \% \mathrm{i}-\mathrm{PrOH}$ in hexanes; $1.0 \mathrm{~mL} / \mathrm{min} ; 35^{\circ} \mathrm{C}$; retention times: $26.2 \mathrm{~min}$ (major), $29.6 \mathrm{~min}$ (minor).

${ }^{1} \mathrm{H}$ NMR (400 MHz, $\left.\mathrm{CDCl}_{3}\right) \delta 7.33-7.23(\mathrm{~m}, 5 \mathrm{H}), 6.70($ br s, $1 \mathrm{H}), 4.40(\mathrm{~d}, J=6.0 \mathrm{~Hz}$, 2H), 3.93 (br s, 1H), 3.88-3.57 (m, 6H), 2.78-2.65 (m, 2H), $2.57(\mathrm{~d}, J=13.6 \mathrm{~Hz}, 1 \mathrm{H})$, $2.46(\mathrm{~d}, J=13.6 \mathrm{~Hz}, 1 \mathrm{H}), 2.08-2.00(\mathrm{~m}, 2 \mathrm{H}) \mathrm{ppm}$.

${ }^{13} \mathrm{C}$ NMR $\left(100 \mathrm{MHz} \mathrm{CDCl}_{3}\right) \delta 170.5,138.0,128.7,127.8,127.5,72.4,66.0,54.2,43.8$, 42.8, 34.3, 29.7, $26.8 \mathrm{ppm}$.

HRMS (CI) Calcd for $\mathrm{C}_{15} \mathrm{H}_{21} \mathrm{NNaO}_{3} \mathrm{~S}[\mathrm{M}+\mathrm{Na}]^{+}$: 318.1140, Found: 318.1208. 


\section{Product Derivatizations}

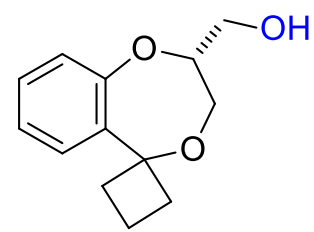

2n

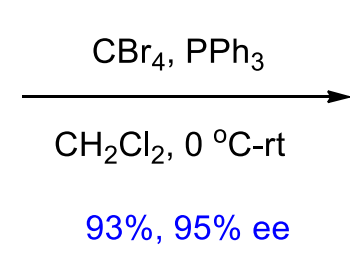

$93 \%, 95 \%$ ee

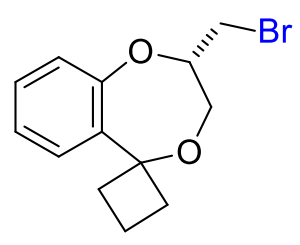

8

(S)-2-(Bromomethyl)-2,3-dihydrospiro[benzo[e][1,4]dioxepine-5,1'-cyclobutane]

(8). Under $\mathrm{N}_{2}$, to a solution of alcohol $2 \mathrm{n}(44.1 \mathrm{mg}, 0.20 \mathrm{mmol})$ and tetrabromomethane $(79.6 \mathrm{mg}, 0.24 \mathrm{mmol})$ in anhydrous $\mathrm{CH}_{2} \mathrm{Cl}_{2}(1.0 \mathrm{~mL})$ at $0{ }^{\circ} \mathrm{C}$ was added dropwise triphenylphosphine $(52.5 \mathrm{mg}, 0.20 \mathrm{mmol})$ in anhydrous $\mathrm{CH}_{2} \mathrm{Cl}_{2}$ (1.0 mL). The reaction mixture was spontaneously warmed to room temperature and stirired overnight. Upon completion, the mixture was diluted with $\mathrm{CH}_{2} \mathrm{Cl}_{2}(15$ $\mathrm{mL}$ ), washed successively with water and brine, dried over anhydrous $\mathrm{Na}_{2} \mathrm{SO}_{4}$, and concentrated under reduced pressure. The residue was purified by silica gel column chromatography (petroleum ether/ethyl acetate $=20: 1$ to $10: 1$ ) to afford the desired product 8 as colorless oil (52.9 mg, 93\% yield).

$[\alpha]_{D^{24}}:-3.9\left(c=2.0, \mathrm{CHCl}_{3}\right) .95 \%$ ee, HPLC analysis of the product: Daicel CHIRALPAK AD-H column; $2 \%$ i-PrOH in hexanes; $1.0 \mathrm{~mL} / \mathrm{min} ; 35^{\circ} \mathrm{C}$; retention times: $7.7 \mathrm{~min}$ (minor), $8.4 \mathrm{~min}$ (major).

${ }^{1} \mathbf{H}$ NMR $\left(400 \mathrm{MHz}, \mathrm{CDCl}_{3}\right) \delta 7.44(\mathrm{~d}, J=7.6 \mathrm{~Hz}, 1 \mathrm{H}), 7.29(\mathrm{t}, J=7.2 \mathrm{~Hz}, 1 \mathrm{H}), 7.19$ $7.13(\mathrm{~m}, 2 \mathrm{H}), 4.10-3.97(\mathrm{~m}, 3 \mathrm{H}), 3.52-3.47(\mathrm{~m}, 1 \mathrm{H}), 3.42\left(\mathrm{dd}, J_{1}=10.8 \mathrm{~Hz}, J_{2}=5.2 \mathrm{~Hz}\right.$, $1 \mathrm{H}), 2.69-2.66(\mathrm{~m}, 1 \mathrm{H}), 2.54(\mathrm{q}, J=6.4 \mathrm{~Hz}, 1 \mathrm{H}), 2.42-2.28(\mathrm{~m}, 2 \mathrm{H}), 1.88-1.81(\mathrm{~m}, 1 \mathrm{H})$, 1.67-1.55 (m, 1H) ppm.

${ }^{13} \mathrm{C}$ NMR $\left(100 \mathrm{MHz}, \mathrm{CDCl}_{3}\right) \delta 156.8,135.9,129.1,126.0,124.1,122.6,81.2,80.8,66.8$, $33.2,33.1,30.8,13.0$ ppm. 
HRMS (CI) Calcd for $\mathrm{C}_{13} \mathrm{H}_{13} \mathrm{BrNaO}_{2}[\mathrm{M}+\mathrm{Na}]^{+}$: 305.0153, Found: 305.0137 .

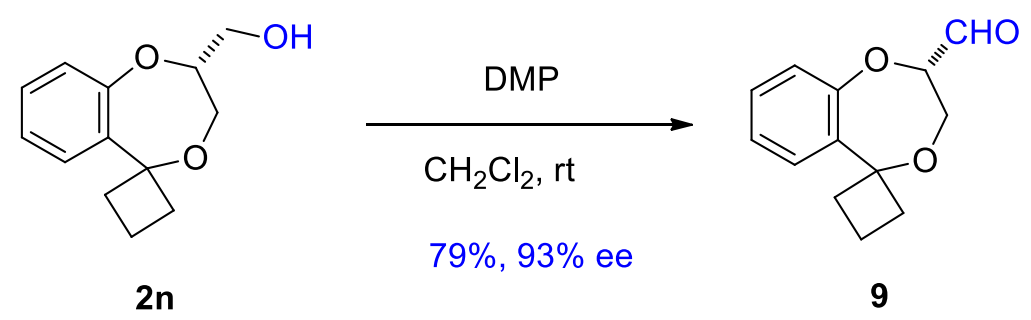

(S)-2,3-Dihydrospiro[benzo[e][1,4]dioxepine-5,1'-cyclobutane]-2-carbaldehyde

(9). To a solution of alcohol $2 \mathbf{n}(44.1 \mathrm{mg}, 0.20 \mathrm{mmol})$ in anhydrous $\mathrm{CH}_{2} \mathrm{Cl}_{2}(2 \mathrm{~mL})$ at $0{ }^{\circ} \mathrm{C}$ was added Dess-Martin periodinane $(169.6 \mathrm{mg}, 0.40 \mathrm{mmol})$ in one portion. The reaction mixture was spontaneously warmed to room temperature and stirired overnight. Upon completion, the mixture was diluted with $\mathrm{CH}_{2} \mathrm{Cl}_{2}$ (15 mL), washed successively with water and brine, dried over anhydrous $\mathrm{Na}_{2} \mathrm{SO}_{4}$, and concentrated under reduced pressure. The residue was purified by silica gel column chromatography (petroleum ether/ethyl acetate $=10: 1$ to 5:1) to afford the desired product 9 as colorless oil (34.5 mg, 79\% yield).

$[\alpha]_{D^{24}}:-31.5\left(c=0.5, \mathrm{CHCl}_{3}\right) .93 \%$ ee, HPLC analysis of the product: Daicel CHIRALCEL OD-H column; 10\% i-PrOH in hexanes; $1.0 \mathrm{~mL} / \mathrm{min}$; retention times: $12.5 \mathrm{~min}$ (minor), $16.1 \mathrm{~min}$ (major).

${ }^{1}$ H NMR $\left(400 \mathrm{MHz}, \mathrm{CDCl}_{3}\right) \delta 9.85(\mathrm{~s}, 1 \mathrm{H}), 7.44(\mathrm{~d}, J=6.8 \mathrm{~Hz}, 1 \mathrm{H}), 7.30-7.26(\mathrm{~m}, 1 \mathrm{H})$, 7.19-7.11 (m, 2H), $4.25\left(\mathrm{dd}, J_{1}=8.4 \mathrm{~Hz}, J_{2}=6.8 \mathrm{~Hz}, 1 \mathrm{H}\right), 4.10\left(\mathrm{dd}, J_{1}=13.2 \mathrm{~Hz}, J_{2}=2.8\right.$ $\mathrm{Hz}, 1 \mathrm{H}), 4.05-4.0(\mathrm{~m}, 1 \mathrm{H}), 2.75-2.68(\mathrm{~m}, 1 \mathrm{H}), 2.55-2.48(\mathrm{~m}, 1 \mathrm{H}), 2.40-2.32(\mathrm{~m}, 1 \mathrm{H})$, 2.25-2.20 (m, 1H), 1.86-1.78 (m, 1H), 1.62-1.50 (m, 1H) ppm. 
${ }^{13} \mathrm{C}$ NMR $\left(100 \mathrm{MHz}, \mathrm{CDCl}_{3}\right) \delta 200.1,156.9,135.3,129.3,126.5,124.5,122.2,85.5,81.5$, 64.0, 33.3, 33.1, 13.0 ppm.

HRMS (CI) Calcd for $\mathrm{C}_{13} \mathrm{H}_{14} \mathrm{NaO}_{3}[\mathrm{M}+\mathrm{Na}]^{+}: 241.0841$, Found: 241.0820 .

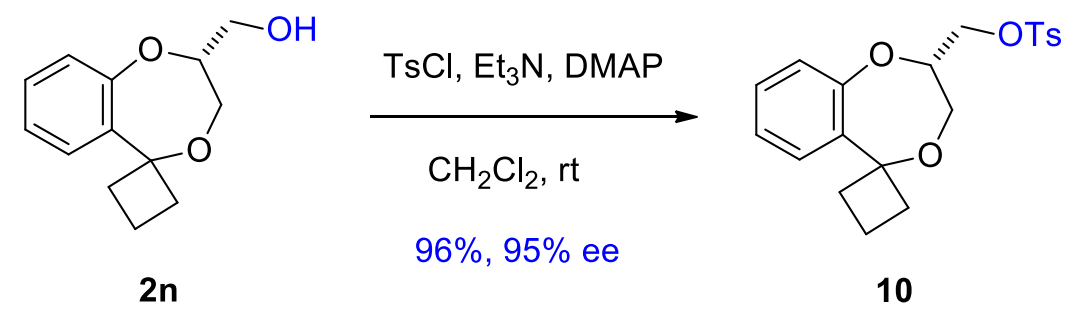

(S)-(2,3-Dihydrospiro[benzo[e][1,4]dioxepine-5,1'-cyclobutan]-2-yl)methyl 4methylbenzenesulfonate (10). To a solution of alcohol 2n (132.2 mg, $0.60 \mathrm{mmol})$, $\mathrm{Et}_{3} \mathrm{~N}(0.25 \mathrm{~mL}, 1.80 \mathrm{mmol})$, and DMAP $(18.3 \mathrm{mg}, 0.15 \mathrm{mmol})$ in anhydrous $\mathrm{CH}_{2} \mathrm{Cl}_{2}$ $(5 \mathrm{~mL})$ at $0{ }^{\circ} \mathrm{C}$ was added $\mathrm{TsCl}(228.7 \mathrm{mg}, 1.20 \mathrm{mmol})$ in one portion. The reaction mixture was spontaneously warmed to room temperature and stirired overnight. Upon completion, the mixture was quenched by saturated $\mathrm{NH}_{4} \mathrm{Cl}$ aqueous solution (5 mL). The layers were separated, and the aqueous layer was extracted with $\mathrm{CH}_{2} \mathrm{Cl}_{2}(2 \times 15 \mathrm{~mL})$. The combined organic layers were washed with brine, dried over anhydrous $\mathrm{Na}_{2} \mathrm{SO}_{4}$, and concentrated under reduced pressure. The residue was purified by silica gel column chromatography (petroleum ether/ethyl acetate $=15: 1$ to $10: 1)$ to afford the desired product 10 as colorless oil $(216.0 \mathrm{mg}, 96 \%$ yield $)$. $[\alpha]_{\mathrm{D}^{25}}:-23.3\left(c=0.5, \mathrm{CHCl}_{3}\right) .95 \%$ ee, HPLC analysis of the product: Daicel CHIRALCEL OD-H column; $5 \%$ - $\mathrm{PrOH}$ in hexanes; $1.0 \mathrm{~mL} / \mathrm{min} ; 35{ }^{\circ} \mathrm{C}$; retention times: $19.3 \mathrm{~min}$ (major), $23.5 \mathrm{~min}$ (minor).

${ }^{1} \mathrm{H}$ NMR $\left(400 \mathrm{MHz}, \mathrm{CDCl}_{3}\right) \delta 7.80(\mathrm{~d}, J=8.0 \mathrm{~Hz}, 2 \mathrm{H}), 7.38-7.33(\mathrm{~m}, 3 \mathrm{H}), 7.19(\mathrm{t}, J=$ $7.6 \mathrm{~Hz}, 1 \mathrm{H}), 7.09$ (t, J = 7.6 Hz, 1H), $6.94(\mathrm{~d}, J=7.6 \mathrm{~Hz}, 1 \mathrm{H}), 4.19-4.15(\mathrm{~m}, 1 \mathrm{H}), 4.09$ 
$4.02(\mathrm{~m}, 2 \mathrm{H}), 3.87-3.86(\mathrm{~m}, 2 \mathrm{H}), 2.64-2.57(\mathrm{~m}, 1 \mathrm{H}), 2.48-2.41(\mathrm{~m}, 4 \mathrm{H}), 2.32(\mathrm{~d}, J=9.6$ $\mathrm{Hz}, 1 \mathrm{H}), 2.23-2.17(\mathrm{~m}, 1 \mathrm{H}), 1.82-1.75(\mathrm{~m}, 1 \mathrm{H}), 1.56-1.49(\mathrm{~m}, 1 \mathrm{H}) \mathrm{ppm}$.

${ }^{13} \mathrm{C}$ NMR $\left(100 \mathrm{MHz}, \mathrm{CDCl}_{3}\right) \delta 156.5,145.1,135.6,132.8,130.0,129.0,128.1,126.0$, $124.0,122.4,81.2,78.5,68.7,65.2,33.2,33.1,21.7,12.9$ ppm.

HRMS (CI) Calcd for $\mathrm{C}_{20} \mathrm{H}_{22} \mathrm{NaO}_{5} \mathrm{~S}[\mathrm{M}+\mathrm{Na}]^{+}$: 397.1086, Found: 397.1062 .

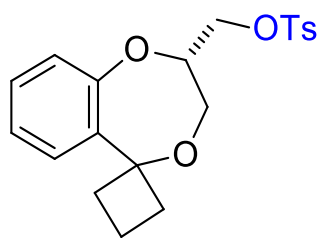

10

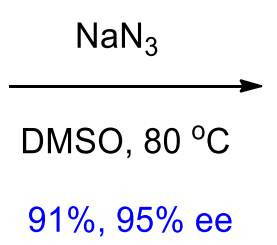

$91 \%, 95 \%$ ee

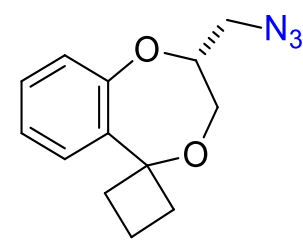

11

(R)-2-(Azidomethyl)-2,3-dihydrospiro[benzo[e][1,4]dioxepine-5,1'-cyclobutane]

(11). To a solution of $\mathbf{1 0}(37.5 \mathrm{mg}, 0.10 \mathrm{mmol})$ in DMSO $(0.5 \mathrm{~mL})$ was added sodium azide $(13.0 \mathrm{mg}, 0.20 \mathrm{mmol})$. After stirring at $80{ }^{\circ} \mathrm{C}$ overnight, the mixture was diluted with EtOAc $(10 \mathrm{~mL})$, and washed successively with $\mathrm{H}_{2} \mathrm{O}(3 \times 5 \mathrm{~mL})$ and brine $(5 \mathrm{~mL})$. The organic layer was dried over anhydrous $\mathrm{Na}_{2} \mathrm{SO}_{4}$, and concentrated under reduced pressure. The residue was purified by silica gel column chromatography (petroleum ether/ethyl acetate $=30: 1$ to $20: 1$ ) to give the desired product $\mathbf{1 1}$ as colorless oil (22.3 mg, 91\% yield).

$[\alpha]_{D^{22}}:-87.9\left(c=0.5, \mathrm{CHCl}_{3}\right) .95 \%$ ee, HPLC analysis of the product: Daicel CHIRALCEL OD-H column; 3\% $i-\mathrm{PrOH}$ in hexanes; $1.0 \mathrm{~mL} / \mathrm{min} ; 35^{\circ} \mathrm{C}$; retention times: $7.9 \mathrm{~min}$ (major), $9.0 \mathrm{~min}$ (minor).

${ }^{1} \mathbf{H}$ NMR $\left(400 \mathrm{MHz}, \mathrm{CDCl}_{3}\right) \delta 7.42(\mathrm{~d}, J=7.6 \mathrm{~Hz}, 1 \mathrm{H}), 7.26(\mathrm{t}, J=8.0 \mathrm{~Hz}, 1 \mathrm{H}), 7.16-$ $7.11(\mathrm{~m}, 2 \mathrm{H}), 4.04-3.92(\mathrm{~m}, 2 \mathrm{H}), 3.84\left(\mathrm{dd}, J_{1}=12.4 \mathrm{~Hz}, J_{2}=5.6 \mathrm{~Hz}, 1 \mathrm{H}\right), 3.52-3.47(\mathrm{~m}$, $1 \mathrm{H}), 3.21\left(\mathrm{dd}, J_{1}=9.2 \mathrm{~Hz}, J_{2}=3.6 \mathrm{~Hz}, 1 \mathrm{H}\right), 2.74-2.67(\mathrm{~m}, 1 \mathrm{H}), 2.58-2.50(\mathrm{~m}, 1 \mathrm{H}), 2.38-$ $2.30(\mathrm{~m}, 1 \mathrm{H}), 2.26-2.19(\mathrm{~m}, 1 \mathrm{H}), 1.87-1.78(\mathrm{~m}, 1 \mathrm{H}), 1.63-1.54(\mathrm{~m}, 1 \mathrm{H}) \mathrm{ppm}$. 
${ }^{13} \mathrm{C}$ NMR $\left(100 \mathrm{MHz}_{2} \mathrm{CDCl}_{3}\right) \delta 157.2,135.9,129.1,126.1,124.0,122.2,81.2,80.8,66.2$, 51.5, 33.1, 33.0, 13.0 ppm.

HRMS (CI) Calcd for $\mathrm{C}_{13} \mathrm{H}_{15} \mathrm{~N}_{3} \mathrm{NaO}_{2}[\mathrm{M}+\mathrm{Na}]^{+}$: 268.1062, Found: 268.1044.<smiles>[O-]C[C@H]1COC2(CCC2)c2ccccc2O1</smiles>

10<smiles>CN1C(=O)c2ccccc2C1=O</smiles>

(1)

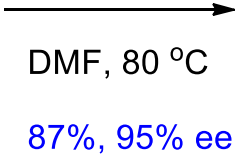

$87 \%, 95 \%$ ee<smiles>O=C1c2ccccc2C(=O)N1C[C@H]1COC2(CCC2)c2ccccc2O1</smiles>

12

(R)-2-((2,3-Dihydrospiro[benzo[e][1,4]dioxepine-5,1'-cyclobutan]-2-yl)methyl)-

isoindoline-1,3-dione (12). To a solution of $10(37.5 \mathrm{mg}, 0.10 \mathrm{mmol})$ in DMF (0.5 $\mathrm{mL}$ ) was added potassium 1,3-dioxoisoindolin-2-ide (37.0 mg, $0.20 \mathrm{mmol})$. After stirring at $80{ }^{\circ} \mathrm{C}$ overnight, the mixture was diluted with EtOAc $(10 \mathrm{~mL})$, and washed successively with $\mathrm{H}_{2} \mathrm{O}(3 \times 5 \mathrm{~mL})$ and brine $(5 \mathrm{~mL})$. The organic layer was dried over anhydrous $\mathrm{Na}_{2} \mathrm{SO}_{4}$, and concentrated under reduced pressure. The residue was purified by silica gel column chromatography (petroleum ether/ethyl acetate $=20: 1$ to $10: 1)$ to give the desired product 12 as a white solid (30.4 mg, 87\% yield).

$[\alpha]_{\mathrm{D}^{22}}:+14.0\left(c=0.5, \mathrm{CHCl}_{3}\right) .95 \%$ ee, HPLC analysis of the product: Daicel CHIRALCEL OD-H column; $5 \% i$-PrOH in hexanes; $1.0 \mathrm{~mL} / \mathrm{min} ; 35^{\circ} \mathrm{C}$; retention times: $18.6 \mathrm{~min}$ (minor), $25.3 \mathrm{~min}$ (major).

${ }^{1} \mathbf{H}$ NMR $\left(400 \mathrm{MHz}, \mathrm{CDCl}_{3}\right) \delta$ 7.89-7.86 (m, 2H), 7.76-7.72 (m, 2H), $7.37(\mathrm{~d}, J=7.6$ $\mathrm{Hz}, 1 \mathrm{H}), 7.13\left(\mathrm{td}, J_{1}=6.0 \mathrm{~Hz}, J_{2}=1.2 \mathrm{~Hz}, 1 \mathrm{H}\right), 7.05(\mathrm{t}, J=7.6 \mathrm{~Hz}, 1 \mathrm{H}), 6.82(\mathrm{~d}, J=8.0$ $\mathrm{Hz}, 1 \mathrm{H}), 4.24-4.18(\mathrm{~m}, 1 \mathrm{H}), 4.04-3.87(\mathrm{~m}, 3 \mathrm{H}), 3.69\left(\mathrm{dd}, J_{1}=14.0 \mathrm{~Hz}, J_{2}=4.8 \mathrm{~Hz}, 1 \mathrm{H}\right)$, 
2.71-2.64 (m, 1H), 2.58-2.50 (m, 1H), 2.35-2.28 (m, 1H), 2.25-2.18 (m, 1H), 1.84-1.75 (m, 1H), 1.55-1.48 (m, 1H) ppm.

${ }^{13} \mathrm{C}$ NMR $\left(100 \mathrm{MHz}, \mathrm{CDCl}_{3}\right) \delta 168.1,157.0,135.4,134.1,132.0,128.9,126.0,123.7$, $123.4,122.0,81.1,78.7,66.4,38.9,33.3,33.1,12.9$ ppm

HRMS (CI) Calcd for $\mathrm{C}_{21} \mathrm{H}_{19} \mathrm{NNaO}_{4}[\mathrm{M}+\mathrm{Na}]^{+}$: 372.1212, Found: 372.1192.

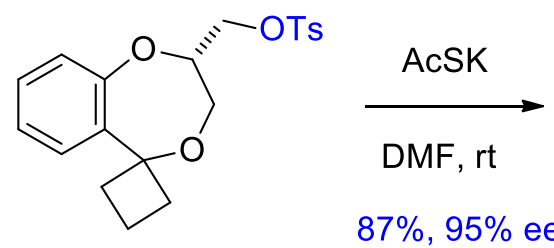

10

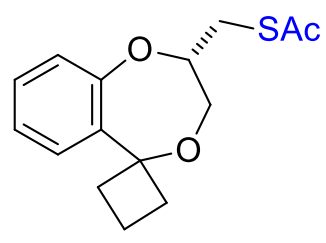

13

(S)-S-((2,3-Dihydrospiro[benzo[e][1,4]dioxepine-5,1'-cyclobutan]-2-yl)methyl)

ethanethioate (13). To a solution of $10(37.5 \mathrm{mg}, 0.10 \mathrm{mmol})$ in DMF $(0.5 \mathrm{~mL})$ was added potassium ethanethioate $(22.8 \mathrm{mg}, 0.20 \mathrm{mmol})$. After stirring at room temperature overnight, the mixture was diluted with EtOAc $(10 \mathrm{~mL})$, and washed successively with $\mathrm{H}_{2} \mathrm{O}(3 \times 5 \mathrm{~mL})$ and brine $(5 \mathrm{~mL})$. The organic layer was dried over anhydrous $\mathrm{Na}_{2} \mathrm{SO}_{4}$, and concentrated under reduced pressure. The residue was purified by silica gel column chromatography (petroleum ether/ethyl acetate $=30: 1)$ to give the desired product 13 as colorless oil (24.2 $\mathrm{mg}, 87 \%$ yield $)$.

$[\alpha]_{\mathrm{D}^{22}}:+48.8\left(c=0.5, \mathrm{CHCl}_{3}\right) .95 \%$ ee, HPLC analysis of the product: Daicel CHIRALCEL OD-H column; 3\% $i-\mathrm{PrOH}$ in hexanes; $1.0 \mathrm{~mL} / \mathrm{min} ; 35^{\circ} \mathrm{C}$; retention times: $10.4 \mathrm{~min}$ (major), $11.2 \mathrm{~min}$ (minor).

${ }^{1} \mathbf{H}$ NMR $\left(400 \mathrm{MHz}, \mathrm{CDCl}_{3}\right) \delta 7.39\left(\mathrm{dd}, J_{1}=8.4 \mathrm{~Hz}, J_{2}=1.6 \mathrm{~Hz}, 1 \mathrm{H}\right), 7.25-7.20(\mathrm{~m}$, 1H), 7.11-7.07 (m, 2H), 3.93-3.90 (m, 2H), 3.88-3.84 (m, 1H), 3.16-3.11 (m, 1H), 3.04$2.98(\mathrm{~m}, 1 \mathrm{H}), 2.70-2.63(\mathrm{~m}, 1 \mathrm{H}), 2.56-2.49(\mathrm{~m}, 1 \mathrm{H}), 2.36(\mathrm{~s}, 3 \mathrm{H}), 2.35-2.28(\mathrm{~m}, 1 \mathrm{H})$, 2.25-2.18 (m, 1H), 1.84-1.76 (m, 1H), 1.60-1.51 (m, 1H) ppm. 
${ }^{13} \mathrm{C}$ NMR $\left(100 \mathrm{MHz}, \mathrm{CDCl}_{3}\right) \delta 195.1,157.3,136.1,129.0,126.0,123.8,122.3,81.1,80.7$, $67.6,33.0,32.9,30.6,30.5,13.0 \mathrm{ppm}$

HRMS (CI) Calcd for $\mathrm{C}_{15} \mathrm{H}_{18} \mathrm{NaO}_{3} \mathrm{~S}[\mathrm{M}+\mathrm{Na}]^{+}$: 301.0874, Found: 301.0862 .

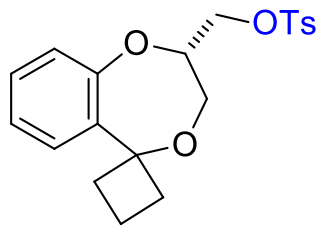

10

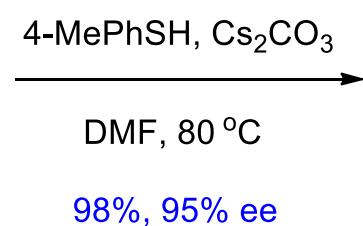

$98 \%, 95 \%$ ee

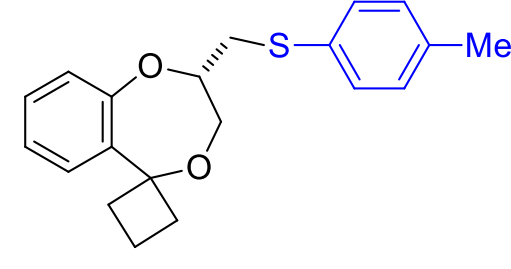

14

(S)-2-((p-Tolylthio)methyl)-2,3-dihydrospiro[benzo[e][1,4]dioxepine-5,1'-cyclo-

butane] (14). To a solution of $10(37.5 \mathrm{mg}, 0.10 \mathrm{mmol})$ in DMF $(0.5 \mathrm{~mL})$ was added 4-methylbenzenethiol $(24.8 \mathrm{mg}, 0.20 \mathrm{mmol})$ and cesium carbonate $(65.2 \mathrm{mg}, 0.20$ mmol). After stirring at $80{ }^{\circ} \mathrm{C}$ overnight, the mixture was diluted with EtOAc $(10$ $\mathrm{mL})$, and washed successively with $\mathrm{H}_{2} \mathrm{O}(3 \times 5 \mathrm{~mL})$ and brine $(5 \mathrm{~mL})$. The organic layer was dried over anhydrous $\mathrm{Na}_{2} \mathrm{SO}_{4}$, and concentrated under reduced pressure. The residue was purified by silica gel column chromatography (petroleum ether/ethyl acetate $=50: 1$ to $20: 1$ ) to give the desired product 14 as pale yellow oil (31.9 mg, 98\% yield).

$[\alpha]_{D^{22}}:+29.6\left(c=0.5, \mathrm{CHCl}_{3}\right) .95 \%$ ee, HPLC analysis of the product: Daicel CHIRALPAK AD-H column; $2 \% \mathrm{i}-\mathrm{PrOH}$ in hexanes; $1.0 \mathrm{~mL} / \mathrm{min} ; 35^{\circ} \mathrm{C}$; retention times: $10.1 \mathrm{~min}$ (minor), $17.0 \mathrm{~min}$ (major).

${ }^{1} \mathbf{H}$ NMR $\left(400 \mathrm{MHz}, \mathrm{CDCl}_{3}\right) \delta 7.39(\mathrm{~d}, J=7.2 \mathrm{~Hz}, 1 \mathrm{H}), 7.30(\mathrm{~d}, J=8.0 \mathrm{~Hz}, 2 \mathrm{H}), 7.23(\mathrm{t}$, $J=7.6 \mathrm{~Hz}, 1 \mathrm{H}), 7.11-7.08(\mathrm{~m}, 4 \mathrm{H}), 4.00-3.92(\mathrm{~m}, 3 \mathrm{H}), 3.16\left(\mathrm{dd}, J_{1}=13.6 \mathrm{~Hz}, J_{2}=6.4\right.$ $\mathrm{Hz}, 1 \mathrm{H}), 2.92\left(\mathrm{dd}, J_{1}=14.0 \mathrm{~Hz}, J_{2}=5.6 \mathrm{~Hz}, 1 \mathrm{H}\right), 2.68-2.61(\mathrm{~m}, 1 \mathrm{H}), 2.52(\mathrm{q}, J=9.6 \mathrm{~Hz}$, 1H), 2.36-2.21 (m, 5H), 1.85-1.77 (m, 1H), 1.64-1.52 (m, 1H) ppm. 
${ }^{13} \mathrm{C}$ NMR $\left(100 \mathrm{MHz}, \mathrm{CDCl}_{3}\right) \delta 157.4,136.8,136.1,132.1,130.7,130.0,129.0,126.0$, $123.8,122.5,81.2,80.4,67.6,36.3,33.1,33.0,21.1,13.0 \mathrm{ppm}$.

HRMS (CI) Calcd for $\mathrm{C}_{20} \mathrm{H}_{22} \mathrm{NaO}_{2} \mathrm{~S}[\mathrm{M}+\mathrm{Na}]^{+}: 349.1238$, Found: 349.1249 .

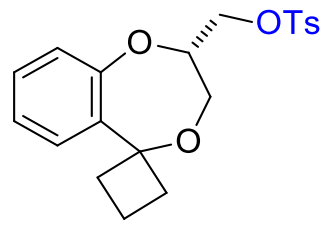

10

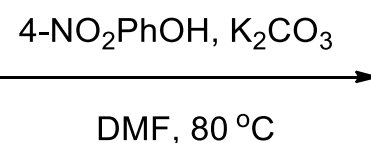

$97 \%, 95 \%$ ee

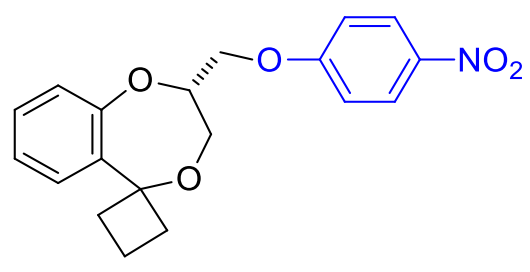

15

(R)-2-((4-Nitrophenoxy)methyl)-2,3-dihydrospiro[benzo[e][1,4]dioxepine-5,1'-

cyclobutane] (15). To a solution of $10(37.5 \mathrm{mg}, 0.10 \mathrm{mmol})$ in DMF (0.5 mL) was added 4-methylbenzenethiol (27.8 $\mathrm{mg}, 0.20 \mathrm{mmol})$ and potassium carbonate $(27.6$ $\mathrm{mg}, 0.20 \mathrm{mmol}$ ). After stirring at $80{ }^{\circ} \mathrm{C}$ overnight, the mixture was diluted with EtOAc $(10 \mathrm{~mL})$, and washed successively with $\mathrm{H}_{2} \mathrm{O}(3 \times 5 \mathrm{~mL})$ and brine $(5 \mathrm{~mL})$. The organic layer was dried over anhydrous $\mathrm{Na}_{2} \mathrm{SO}_{4}$, and concentrated under reduced pressure. The residue was purified by silica gel column chromatography (petroleum ether/ethyl acetate $=10: 1$ ) to give the desired product 15 as a pale yellow solid (33.1 mg, 97\% yield).

$[\alpha]_{D^{22}}:-36.8\left(c=0.5, \mathrm{CHCl}_{3}\right) .95 \%$ ee, HPLC analysis of the product: Daicel CHIRALCEL OD-H column; 5\% $i-\mathrm{PrOH}$ in hexanes; $1.0 \mathrm{~mL} / \mathrm{min} ; 35^{\circ} \mathrm{C}$; retention times: 24.5 min (major), 30.8 min (minor).

${ }^{1} \mathbf{H}$ NMR $\left(400 \mathrm{MHz} \mathrm{CDCl}_{3}\right) \delta 8.20(\mathrm{~d}, J=9.2 \mathrm{~Hz}, 2 \mathrm{H}), 7.43\left(\mathrm{dd}, J_{1}=7.6 \mathrm{~Hz}, J_{2}=0.8\right.$ $\mathrm{Hz}, 1 \mathrm{H}), 7.23\left(\mathrm{td}, J_{1}=7.6 \mathrm{~Hz}, J_{2}=1.6 \mathrm{~Hz}, 1 \mathrm{H}\right), 7.12\left(\mathrm{td}, J_{1}=7.6 \mathrm{~Hz}, J_{2}=0.8 \mathrm{~Hz}, 1 \mathrm{H}\right)$, $7.06(\mathrm{~d}, J=7.6 \mathrm{~Hz}, 1 \mathrm{H}), 6.98(\mathrm{~d}, J=9.2 \mathrm{~Hz}, 2 \mathrm{H}), 4.26(\mathrm{q}, J=6.4 \mathrm{~Hz}, 2 \mathrm{H}), 4.12(\mathrm{q}, J=$ 
$7.2 \mathrm{~Hz}, 1 \mathrm{H}), 4.05(\mathrm{~d}, J=4.4 \mathrm{~Hz}, 2 \mathrm{H}), 2.70-2.63(\mathrm{~m}, 1 \mathrm{H}), 2.58-2.50(\mathrm{~m}, 1 \mathrm{H}), 2.43-2.36$ (m, 1H), 2.34-2.26 (m, 1H), 1.87-1.79 (m, 1H), 1.67-1.54 (m, 1H) ppm.

${ }^{13} \mathrm{C}$ NMR $\left(100 \mathrm{MHz}, \mathrm{CDCl}_{3}\right) \delta 163.6,156.8,141.9,135.8,129.1,126.2,126.0,124.1$, $122.4,114.7,81.4,79.2,68.2,65.7,33.4,33.1,13.0 \mathrm{ppm}$.

HRMS (CI) Calcd for $\mathrm{C}_{19} \mathrm{H}_{19} \mathrm{NNaO}_{5}[\mathrm{M}+\mathrm{Na}]^{+}: 364.1161$, Found: 364.1186. 


\section{Determination of the Absolute Stereochemistry}

The absolute stereochemistry of product $\mathbf{2 e}$ was determined by X-ray diffraction. The X-ray data of $\mathbf{2 e}$ have been deposited at the Cambridge Crystallographic Data Center (CCDC 1967687). The stereochemistry of other products was assumed by analogy.

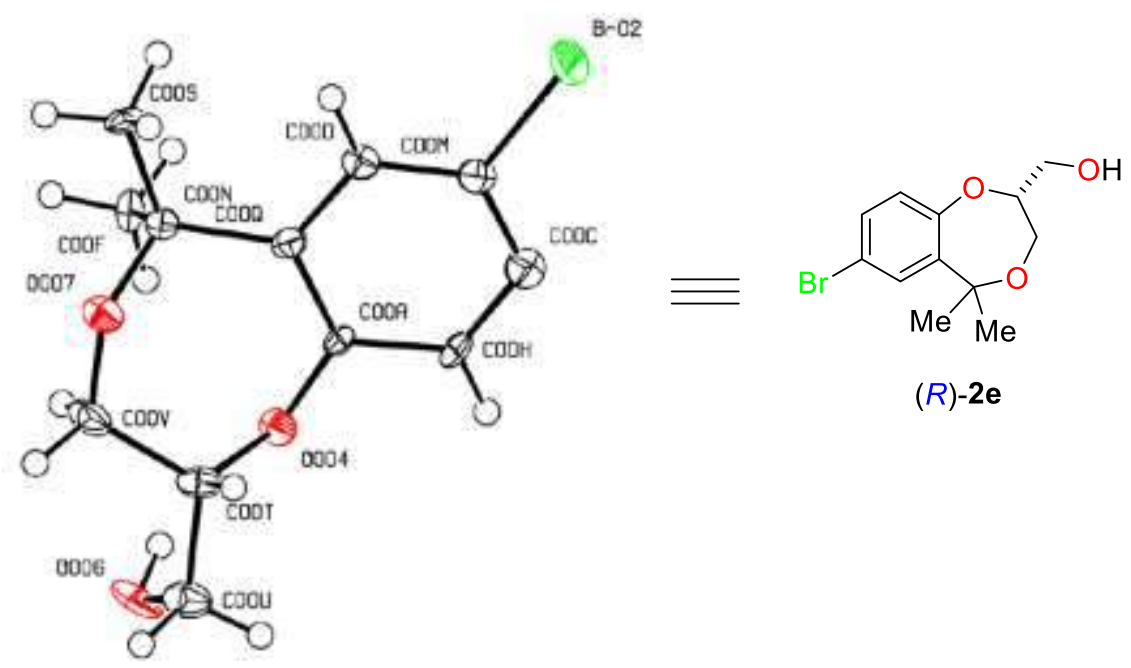

Table 1 Crystal data and structure refinement for $2 \mathrm{e}$.

Identification code 2e

Empirical formula

$\mathrm{C}_{24} \mathrm{H}_{28} \mathrm{Br}_{2} \mathrm{O}_{6}$

Formula weight

572.28

Temperature/K

273.15

Crystal system

monoclinic

Space group

$\mathrm{P} 21$

$\mathrm{a} / \AA$

6.1264(19)

$\mathrm{b} / \AA$

7.0600(17)

c/Å

28.102(8)

$\alpha /^{\circ}$

90

$\beta /{ }^{\circ}$

93.825(9)

$\gamma /{ }^{\circ}$

90

Volume $/ \AA^{3}$

1212.8(6) 
Z

$\rho_{\text {calcg }} / \mathrm{cm}^{3}$

$\mu / \mathrm{mm}^{-1}$

$\mathrm{F}(000)$

Crystal size $/ \mathrm{mm}^{3}$

Radiation

$2 \Theta$ range for data collection $/{ }^{\circ} 4.358$ to 50.786

Index ranges

Reflections collected

Independent reflections

Data/restraints/parameters

Goodness-of-fit on $\mathrm{F}^{2}$

Final R indexes $[I>=2 \sigma(I)]$

$-7 \leq \mathrm{h} \leq 7,-8 \leq \mathrm{k} \leq 8,-33 \leq 1 \leq 33$

15727

$4448\left[R_{\text {int }}=0.0485, R_{\text {sigma }}=0.0582\right]$

$4448 / 1 / 295$

1.100

Final $\mathrm{R}$ indexes [all data]

Largest diff. peak/hole / e $\AA^{-3}$

Flack parameter

$\mathrm{R}_{1}=0.0576, \mathrm{wR}_{2}=0.1450$

$\mathrm{R}_{1}=0.0624, \mathrm{wR}_{2}=0.1474$

$1.68 /-1.08$

$0.066(7)$

Table 2 Fractional Atomic Coordinates $\left(\times 10^{4}\right)$ and Equivalent Isotropic Displacement Parameters $\left(\AA^{2} \times 10^{3}\right)$ for $2 \mathrm{e}$. U $\mathrm{U}_{\text {eq }}$ is defined as $1 / 3$ of of the trace of the orthogonalised $\mathrm{U}_{\mathrm{IJ}}$ tensor.

\begin{tabular}{lrrrr} 
Atom & \multicolumn{1}{c}{$\boldsymbol{x}$} & \multicolumn{1}{c}{$\boldsymbol{y}$} & \multicolumn{1}{c}{$\boldsymbol{z}$} & \multicolumn{1}{c}{$\mathbf{U}(\mathbf{e q})$} \\
Br01 & $9609.8(18)$ & $-2234.8(14)$ & $4740.0(4)$ & \multicolumn{1}{c}{$31.1(3)$} \\
Br02 & $4745.8(19)$ & $12240.1(14)$ & $260.8(4)$ & $32.5(3)$ \\
O003 & $4334(10)$ & $3942(10)$ & $3737(2)$ & $16.3(15)$ \\
O004 & $128(11)$ & $6042(10)$ & $1267(3)$ & $19.1(15)$ \\
O005 & $2055(11)$ & $6984(12)$ & $3283(3)$ & $27.1(17)$ \\
O006 & $-1893(12)$ & $3028(11)$ & $1737(3)$ & $29.4(18)$ \\
O007 & $4545(11)$ & $5522(10)$ & $1762(3)$ & $20.4(15)$ \\
O008 & $8485(11)$ & $4514(10)$ & $3262(3)$ & $20.4(15)$ \\
C009 & $3622(15)$ & $7209(16)$ & $3679(4)$ & $24(2)$ \\
C00A & $1287(13)$ & $7444(14)$ & $1057(3)$ & $12.4(17)$ \\
C00B & $6025(17)$ & $308(15)$ & $4603(4)$ & $22(2)$ \\
C00C & $1270(18)$ & $9666(16)$ & $403(4)$ & $25(2)$ \\
C00D & $4256(15)$ & $9611(14)$ & $1012(3)$ & $16(2)$ \\
C00E & $5601(13)$ & $2524(14)$ & $3948(3)$ & $16.4(19)$ \\
C00F & $2625(16)$ & $8170(15)$ & $2127(3)$ & $20(2)$ \\
C00G & $4856(16)$ & $1753(15)$ & $4359(4)$ & $19(2)$
\end{tabular}




$\begin{array}{lrrrr}\mathrm{C00H} & 274(15) & 8249(15) & 642(3) & 17(2) \\ \mathrm{C} 00 \mathrm{I} & 6201(15) & 1945(15) & 2862(3) & 20(2) \\ \mathrm{C} 00 \mathrm{~J} & 8069(13) & 2459(14) & 3247(3) & 15.4(19) \\ \mathrm{C} 00 \mathrm{~K} & 7911(17) & -318(14) & 4417(4) & 20(2) \\ \mathrm{C} 00 \mathrm{~L} & 8612(15) & 383(14) & 3983(3) & 16.0(19) \\ \mathrm{C} 00 \mathrm{M} & 3280(17) & 10311(14) & 587(3) & 19(2) \\ \mathrm{C} 00 \mathrm{~N} & 4198(14) & 7541(14) & 1757(3) & 18.0(19) \\ \mathrm{C} 00 \mathrm{O} & 6665(17) & 5772(16) & 3240(4) & 24(2) \\ \mathrm{C} 00 \mathrm{P} & 5414(16) & 5737(14) & 3685(4) & 20(2) \\ \mathrm{C} 00 \mathrm{Q} & 3265(15) & 8185(13) & 1264(3) & 17(2) \\ \mathrm{C} 00 \mathrm{R} & 7455(16) & 1790(14) & 3740(3) & 17(2) \\ \mathrm{C} 00 \mathrm{~S} & 6457(15) & 8340(15) & 1886(4) & 19(2) \\ \mathrm{C} 00 \mathrm{~T} & 1248(16) & 4265(14) & 1343(4) & 21(2) \\ \mathrm{C} 00 \mathrm{U} & -557(15) & 2787(16) & 1346(4) & 26(2) \\ \mathrm{C} 00 \mathrm{~V} & 2737(17) & 4297(15) & 1799(4) & 27(2) \\ \mathrm{C} 00 \mathrm{~W} & 10204(16) & 1670(15) & 3093(4) & 24(2)\end{array}$

Table 3 Anisotropic Displacement Parameters $\left(\AA^{2} \times 10^{3}\right)$ for $2 f$. The Anisotropic displacement factor exponent takes the form: $-2 \pi^{2}\left[h^{2} a^{* 2} U_{11}+2 h k a * b * U_{12}+\ldots\right]$.

\begin{tabular}{lrrrrrr} 
Atom & \multicolumn{1}{c}{$\mathbf{U}_{\mathbf{1 1}}$} & \multicolumn{1}{c}{$\mathbf{U}_{\mathbf{2 2}}$} & \multicolumn{1}{c}{$\mathbf{U}_{33}$} & \multicolumn{1}{c}{$\mathbf{U}_{\mathbf{2 3}}$} & \multicolumn{1}{c}{$\mathbf{U} \mathbf{3}$} & \multicolumn{1}{c}{$\mathbf{U 1 2}$} \\
Br01 & $35.1(6)$ & $31.0(7)$ & $27.4(6)$ & $11.1(4)$ & $4.5(4)$ & $10.7(5)$ \\
Br02 & $37.0(6)$ & $31.9(7)$ & $28.3(6)$ & $12.2(5)$ & $-0.8(4)$ & $-9.9(5)$ \\
O003 & $13(3)$ & $17(4)$ & $19(4)$ & $1(3)$ & $2(3)$ & $1(3)$ \\
O004 & $15(3)$ & $18(4)$ & $25(4)$ & $2(3)$ & $0(3)$ & $2(3)$ \\
O005 & $19(3)$ & $23(4)$ & $39(4)$ & $8(3)$ & $-2(3)$ & $1(3)$ \\
O006 & $24(4)$ & $14(4)$ & $51(5)$ & $9(3)$ & $10(3)$ & $-5(3)$ \\
O007 & $14(3)$ & $18(4)$ & $30(4)$ & $3(3)$ & $2(3)$ & $0(3)$ \\
O008 & $12(3)$ & $15(4)$ & $35(4)$ & $2(3)$ & $1(3)$ & $-3(3)$ \\
C009 & $19(5)$ & $18(5)$ & $35(6)$ & $0(5)$ & $4(4)$ & $6(5)$ \\
C00A & $11(4)$ & $17(5)$ & $9(4)$ & $-3(4)$ & $1(3)$ & $-1(4)$ \\
C00B & $23(5)$ & $21(5)$ & $23(5)$ & $0(4)$ & $6(4)$ & $2(4)$ \\
C00C & $25(6)$ & $24(6)$ & $25(6)$ & $0(4)$ & $0(4)$ & $0(5)$ \\
C00D & $11(4)$ & $18(5)$ & $21(5)$ & $0(4)$ & $3(4)$ & $0(4)$ \\
C00E & $16(4)$ & $16(5)$ & $17(4)$ & $-7(4)$ & $0(3)$ & $-3(4)$ \\
C00F & $22(5)$ & $23(5)$ & $14(5)$ & $4(4)$ & $-1(4)$ & $4(4)$ \\
C00G & $13(4)$ & $22(6)$ & $24(5)$ & $-2(4)$ & $8(4)$ & $3(4)$ \\
C00H & $12(4)$ & $24(6)$ & $16(5)$ & $-2(4)$ & $-6(3)$ & $1(4)$ \\
C00I & $15(5)$ & $23(5)$ & $21(5)$ & $-1(4)$ & $-1(4)$ & $-3(4)$ \\
C00J & $14(4)$ & $16(5)$ & $17(4)$ & $4(4)$ & $4(3)$ & $-5(4)$ \\
& & & & & &
\end{tabular}




$\begin{array}{lrrrrrr}\text { C00K } & 23(5) & 17(5) & 19(5) & 4(4) & 1(4) & 2(4) \\ \text { C00L } & 9(4) & 17(5) & 22(5) & -2(4) & 0(4) & -2(4) \\ \text { C00M } & 22(5) & 19(5) & 17(5) & -2(4) & 5(4) & -3(4) \\ \text { C00N } & 18(4) & 18(5) & 18(4) & 2(4) & -1(3) & -4(4) \\ \text { C00O } & 23(5) & 18(5) & 31(6) & 9(4) & 6(4) & 2(4) \\ \text { C00P } & 15(5) & 15(5) & 29(6) & -3(4) & 0(4) & -1(4) \\ \text { C00Q } & 16(4) & 15(5) & 19(5) & -3(4) & -2(4) & 0(4) \\ \text { C00R } & 18(5) & 17(5) & 17(5) & -6(4) & 4(4) & -4(4) \\ \text { C00S } & 12(4) & 21(5) & 23(5) & -3(4) & -2(4) & -9(4) \\ \text { C00T } & 13(5) & 17(5) & 32(6) & -5(4) & 4(4) & -2(4) \\ \text { C00U } & 19(5) & 19(5) & 40(6) & -3(5) & 2(4) & 0(5) \\ \text { C00V } & 21(5) & 14(5) & 45(7) & 8(5) & -6(5) & 0(4) \\ \text { C00W } & 17(5) & 23(6) & 34(6) & 1(4) & 11(4) & 3(4)\end{array}$

Table 4 Bond Lengths for $2 \mathrm{e}$.

$\begin{array}{llll}\text { Atom Atom } & \text { Length/A } & \text { Atom Atom } & \text { Length/A } \\ \text { Br01 C00K } & 1.901(10) & \text { C00C C00H } & 1.373(15) \\ \text { Br02 C00M } & 1.900(10) & \text { C00C C00M } & 1.380(15) \\ \text { O003 C00E } & 1.378(12) & \text { C00DC00M } & 1.390(14) \\ \text { O003 C00P } & 1.442(12) & \text { C00DC00Q } & 1.394(14) \\ \text { O004 C00A } & 1.374(11) & \text { C00E C00G } & 1.381(13) \\ \text { O004 C00T } & 1.439(12) & \text { C00E C00R } & 1.411(13) \\ \text { O005 C009 } & 1.430(12) & \text { C00F C00N } & 1.529(13) \\ \text { O006 C00U } & 1.424(13) & \text { C00I C00J } & 1.564(12) \\ \text { O007 C00N } & 1.441(12) & \text { C00J C00R } & 1.534(12) \\ \text { O007 C00V } & 1.415(13) & \text { C00J C00W } & 1.510(13) \\ \text { O008 C00J } & 1.474(12) & \text { C00K C00L } & 1.409(13) \\ \text { O008 C00O } & 1.424(12) & \text { C00L C00R } & 1.376(14) \\ \text { C009 C00P } & 1.511(14) & \text { C00NC00Q } & 1.533(13) \\ \text { C00A C00H } & 1.403(13) & \text { C00NC00S } & 1.516(12) \\ \text { C00A C00Q } & 1.410(12) & \text { C00OC00P } & 1.508(14) \\ \text { C00B C00G } & 1.399(15) & \text { C00T C00U } & 1.521(14) \\ \text { C00B C00K } & 1.373(14) & \text { C00T C00V } & 1.523(15)\end{array}$

Table 5 Bond Angles for 2e. 


C00A O004 C00T
C00V O007 C00N
C00O O008 C00J
O005 C009 C00P
O004 C00A C00H
O004 C00A C00Q
C00H C00A C00Q
C00K C00B C00G
C00H C00C C00M
C00M C00D C00Q
O003 C00E C00G
O003 C00E C00R
C00G C00E C00R
C00E C00G C00B
C00C C00H C00A
O008 C00J C00I
O008 C00J C00R
O008 C00J C00W
C00R C00J C00I
C00W C00J C00I
C00W C00J C00R
C00B C00K Br01
C00B C00K C00L
C00L C00K Br01

C00A O004 C00T

C00V O007 C00N

C00O O008 C00J

O005 C009 C00P

$\mathrm{O} 004 \mathrm{COOACOOH}$

COOH COOA COOQ

$\mathrm{COOH} \mathrm{COOA} \mathrm{COOQ}$

COOK COOB COOG

$\mathrm{COOH} \mathrm{COOC} \mathrm{COOM}$

C00M C00D C00Q

OOO3 COOE COOR

O003 CO0E C00R

COOG COOE COOR

C00E CO0GCOOB

COOC COOHCOOA

O008 COOJ COOI

O008 C00J C00R

O008 C00J C00W

COOR C00J COOI

COOW COOJ C00I

COOW COOJ COOR

C00L C00K Br01

\begin{tabular}{|c|c|c|}
\hline $115.9(7)$ & C00C C00M Br02 & $119.6(8)$ \\
\hline $119.3(7)$ & C00C C00MC00D & $121.8(9)$ \\
\hline $118.7(7)$ & C00D C00MBr02 & $118.6(7)$ \\
\hline $112.4(9)$ & O007 C00N C00F & $112.4(8)$ \\
\hline $115.8(7)$ & O007 C00N C00Q & $110.4(8)$ \\
\hline $23.0(8)$ & O007 C00N CO0S & $103.5(7)$ \\
\hline $20.9(9)$ & COOF C00N CO0Q & $108.3(7)$ \\
\hline $117.6(9)$ & COOS COON COOF & $109.7(8)$ \\
\hline $118.1(10)$ & COOS COON COOQ & $112.6(8)$ \\
\hline $121.1(9)$ & O008 C00O C00P & $113.2(8)$ \\
\hline $116.1(8)$ & O003 C00P C009 & $105.5(8)$ \\
\hline $122.2(8)$ & $\mathrm{O} 003 \mathrm{C} 00 \mathrm{P} \mathrm{C} 00 \mathrm{O}$ & $111.1(8)$ \\
\hline $121.5(9)$ & C00OC00P C009 & $112.9(9)$ \\
\hline $120.7(9)$ & COOACOOQ COON & $121.4(8)$ \\
\hline $121.1(9)$ & COODC00Q C00A & $116.7(9)$ \\
\hline $111.5(7)$ & COODC00Q CO0N & $121.7(8)$ \\
\hline $109.2(7)$ & COOE COOR COOJ & $121.0(9)$ \\
\hline $102.7(7)$ & COOL COOR COOE & $117.4(9)$ \\
\hline $109.9(7)$ & COOL COOR COOJ & $121.5(8)$ \\
\hline $09.0(8)$ & O004 C00T C00U & $105.1(8)$ \\
\hline $114.4(8)$ & O004 C00T C00V & $111.4(8)$ \\
\hline $19.7(8)$ & COOUC00T C00V & $113.6(9)$ \\
\hline $122.0(9)$ & O006 C00U C00T & $112.2(9)$ \\
\hline $118.3(7)$ & O007 C00V C00T & $112.1(9)$ \\
\hline
\end{tabular}

Table 6 Torsion Angles for 2e.

\begin{tabular}{|c|c|c|c|c|c|c|}
\hline $\mathbf{A}$ & $\mathbf{C}$ & Angle $/^{\circ}$ & $\mathbf{A}$ & B & D & Angle $/^{\circ}$ \\
\hline $\mathrm{Br} 01$ & COOK COOL COOR & $-179.0(7)$ & $\mathrm{COC}$ & $\mathrm{COO}$ & COOMCOOD & $-2.3(16)$ \\
\hline O003 & C00E C00G C00B & $-179.8(9)$ & COOI & $\mathrm{COOJ}$ & CO0R C00E & $-62.2(11)$ \\
\hline $200 ?$ & COOE COOR COOJ & $-3.9(13)$ & COOI & COOJ & COOR COOL & $114.1(10)$ \\
\hline OQ & COOE COOR COOL & $179.6(8)$ & $\mathrm{COOJ}$ & O008 & C00O COOP & $69.9(12)$ \\
\hline 00 & $\mathrm{COOACOOH} \mathrm{COOC}$ & $179.7(9)$ & $\mathrm{COOK}$ & -400 & C00G C00E & $-0.7(16)$ \\
\hline 00 & COOA CO0Q COOD & $-179.7(8)$ & $\mathrm{COOK}$ & $\cos$ & 00R COOE & $1.8(14)$ \\
\hline 0004 & COOA COOQ COON & $-3.4(14)$ & $\mathrm{COOK}$ & $\mathrm{COOL}$ & COOR COOJ & $-174.7(9)$ \\
\hline $\mathrm{O} 00$ & C00T C00U O006 & $-64.7(11)$ & $\mathrm{COOM}$ & $\mathrm{I} C \mathrm{COC}$ & $\mathrm{COOH} \mathrm{COOA}$ & $-1.5(15)$ \\
\hline مOQ & C00T C00V O007 & $-68.7(11)$ & $\mathrm{COON}$ & $\mathrm{COODO}$ & COOQ COOA & $2.6(14)$ \\
\hline $\mathrm{O} 00$ & C009 C00P O003 & $-62.6(10)$ & $\mathrm{COOI}$ & $\mathrm{COOD}$ & C00Q C00N & $-173.7(9)$ \\
\hline $\mathrm{OOO}$ & C009 C00P C00O & $59.0(12)$ & $\mathrm{COO}$ & $\mathrm{O} 00$ & C00V C00T & $71.4(12)$ \\
\hline O007 & COONCOOQ COOA & $58.1(11)$ & $\mathrm{COOO}$ & O008 & $\mathrm{COOJ} \mathrm{COOI}$ & $43.1(11)$ \\
\hline
\end{tabular}




$\begin{array}{lrllr}\text { O007 C00N C00Q C00D } & -125.9(9) & \text { C00O O008 C00J C00R } & -78.5(10) \\ \text { O008 C00J C00R C00E } & 60.3(11) & \text { C00O O008 C00J C00W } & 159.7(8) \\ \text { O008 C00J C00R C00L } & -123.3(9) & \text { C00P O003 C00E C00G } & 121.3(9) \\ \text { O008 C00O C00P O003 } & -67.3(11) & \text { C00P O003 C00E C00R } & -63.7(11) \\ \text { O008 C00O C00P C009 } & 174.4(8) & \text { C00Q C00A C00H C00C } & 5.9(14) \\ \text { C00A O004 C00T C00U } & -153.9(8) & \text { C00Q C00D C00M Br02 } & -179.5(7) \\ \text { C00A O004 C00T C00V } & 82.7(10) & \text { C00Q C00D C00MC00C } & 1.7(15) \\ \text { C00B C00K C00L C00R } & 2.6(15) & \text { C00R C00E C00G C00B } & 5.1(15) \\ \text { C00E O003 C00P C009 } & -153.4(8) & \text { C00S C00NC00Q C00A } & 173.3(9) \\ \text { C00E O003 C00P C00O } & 83.9(10) & \text { C00S C00NC00Q C00D } & -10.7(13) \\ \text { C00F C00N C00Q C00A } & -65.3(11) & \text { C00T O004 C00A C00H } & 125.1(9) \\ \text { C00F C00N C00Q C00D } & 110.7(10) & \text { C00T O004 C00A C00Q } & -61.2(12) \\ \text { C00G C00B C00K Br01 } & 178.5(8) & \text { C00U C00T C00V O007 } & 172.9(8) \\ \text { C00G C00B C00K C00L } & -3.2(16) & \text { C00V O007 C00N C00F } & 43.0(11) \\ \text { C00G C00E C00R C00J } & 170.9(9) & \text { C00V O007 C00N C00Q } & -78.0(10) \\ \text { C00G C00E C00R C00L } & -5.6(14) & \text { C00V O007 C00N C00S } & 161.3(9) \\ \text { C00H C00A C00Q C00D } & -6.3(13) & \text { C00V C00T C00U O006 } & 57.2(12) \\ \text { C00H C00A C00Q C00N } & 169.9(9) & \text { C00W C00J C00R C00E } & 174.8(9) \\ \text { C00HC00C C00MBr02 } & 178.9(8) & \text { C00W C00J C00R C00L } & -8.8(13)\end{array}$

Table 7 Hydrogen Atom Coordinates $\left(\AA \times 10^{4}\right)$ and Isotropic Displacement Parameters $\left(\AA^{2} \times 10^{3}\right)$ for $2 \mathrm{e}$.

\begin{tabular}{lrrrr} 
Atom & \multicolumn{1}{c}{$\boldsymbol{y}$} & $\boldsymbol{z}$ & $\mathbf{U}(\mathbf{e q})$ \\
H005 & 1166 & 6167 & 3345 & 41 \\
H006 & -2073 & 4161 & 1785 & 44 \\
H00A & 2881 & 7121 & 3973 & 29 \\
H00B & 4270 & 8460 & 3666 & 29 \\
H00M & 5592 & 10103 & 1130 & 20 \\
H00N & 1241 & 7550 & 2065 & 30 \\
H00O & 2424 & 9517 & 2107 & 30 \\
H00Q & 3222 & 7839 & 2440 & 30 \\
H00G & 3564 & 2198 & 4475 & 23 \\
H00R & -1096 & 7814 & 528 & 30 \\
H00C & 4867 & 2547 & 2941 & 30 \\
H00D & 5998 & 596 & 2854 & 30 \\
H00E & 6590 & 2375 & 2555 & 29 \\
H00L & 9866 & -109 & 3861 & 29 \\
H00F & 5682 & 5436 & 2969 & 3190 \\
H00H & 7181 & 7051 & &
\end{tabular}




$\begin{array}{lrrrr}\text { H00P } & 6433 & 5950 & 3963 & 24 \\ \text { H00S } & 7007 & 7837 & 2188 & 28 \\ \text { H00T } & 6373 & 9695 & 1906 & 28 \\ \text { H00U } & 7423 & 7995 & 1645 & 28 \\ \text { H00V } & 2135 & 4019 & 1072 & 25 \\ \text { H00W } & -1464 & 2870 & 1050 & 31 \\ \text { H00X } & 96 & 1535 & 1362 & 31 \\ \text { H00Y } & 1902 & 4705 & 2061 & 33 \\ \text { H } & 3264 & 3025 & 1868 & 33 \\ \text { H00I } & 10527 & 2224 & 2794 & 36 \\ \text { H00J } & 10084 & 321 & 3058 & 36 \\ \text { H00K } & 11356 & 1964 & 3330 & 36\end{array}$

\section{Crystal structure determination of $2 \mathrm{e}$}

Crystal Data for $\mathrm{C}_{24} \mathrm{H}_{28} \mathrm{Br}_{2} \mathrm{O}_{6}(M=572.28 \mathrm{~g} / \mathrm{mol})$ : monoclinic, space group $\mathrm{P} 2{ }_{1}$ (no. 4), $a=6.1264(19) \AA, b=7.0600(17) \AA, c=28.102(8) \AA, \beta=93.825(9)^{\circ}, V=1212.8(6) \AA^{3}$, $Z=2, T=273.15 \mathrm{~K}, \mu(\mathrm{MoK} \alpha)=3.379 \mathrm{~mm}^{-1}$, Dcalc $=1.567 \mathrm{~g} / \mathrm{cm}^{3}, 15727$ reflections measured $\left(4.358^{\circ} \leq 2 \Theta \leq 50.786^{\circ}\right), 4448$ unique $\left(R_{\text {int }}=0.0485, \mathrm{R}_{\text {sigma }}=0.0582\right)$ which were used in all calculations. The final $R_{1}$ was $0.0576(\mathrm{I}>2 \sigma(\mathrm{I}))$ and $w R_{2}$ was 0.1474 (all data). 


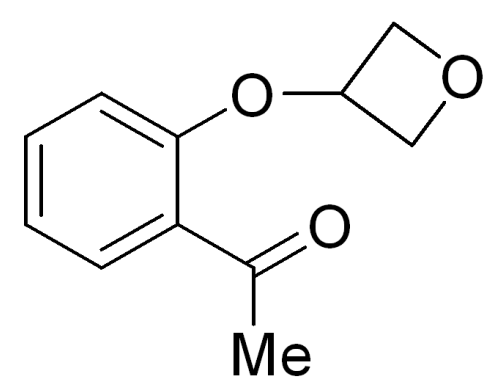

S2

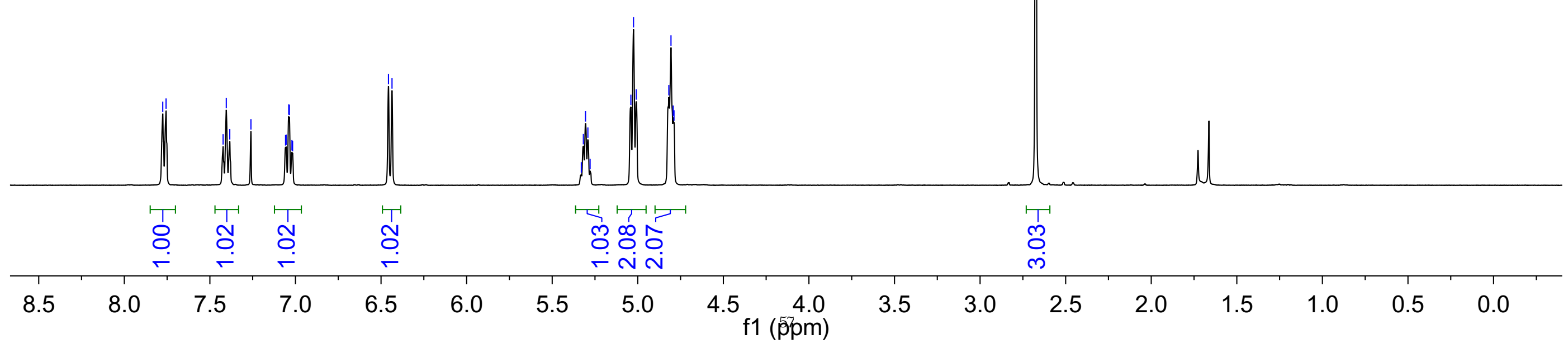


<smiles>CC(=O)c1ccccc1OC1COC1</smiles>

S2

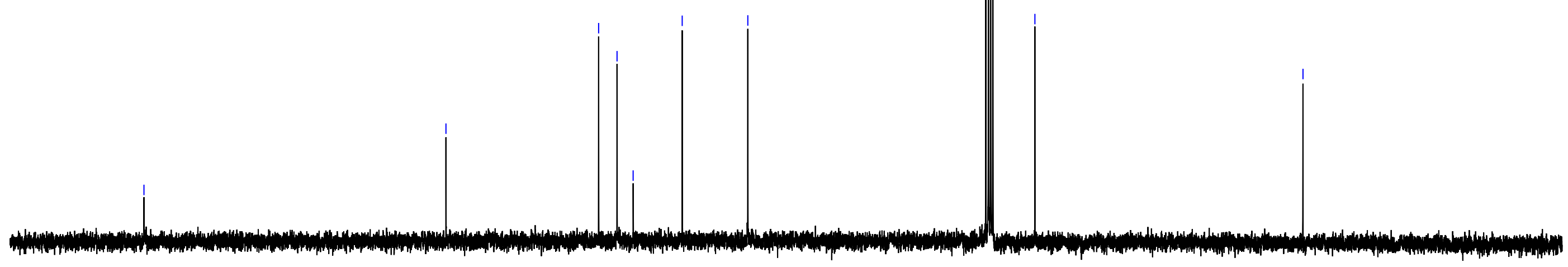




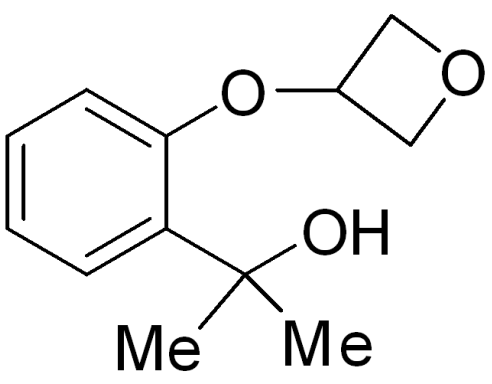

1b

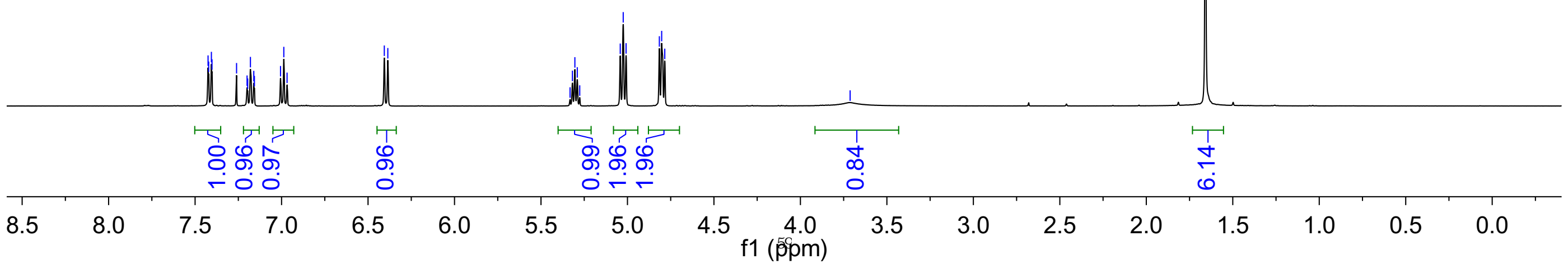


<smiles>CC(C)(O)c1ccccc1OC1COC1</smiles>

$1 b$ 


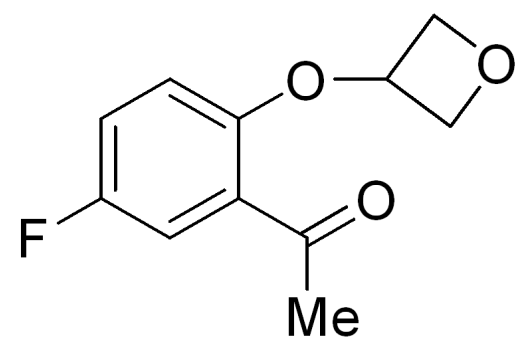

S3

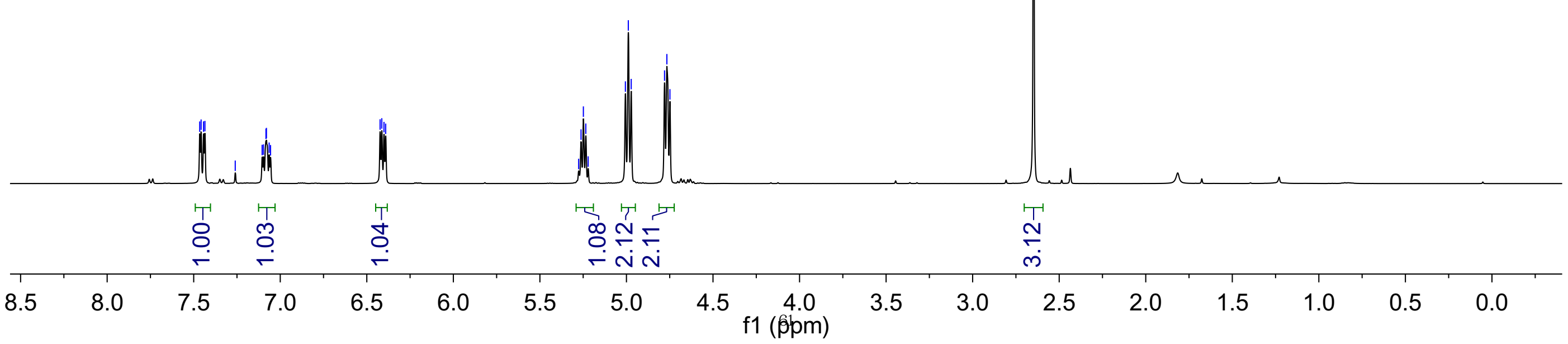




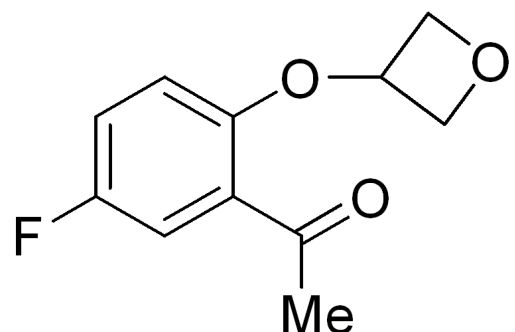

S3

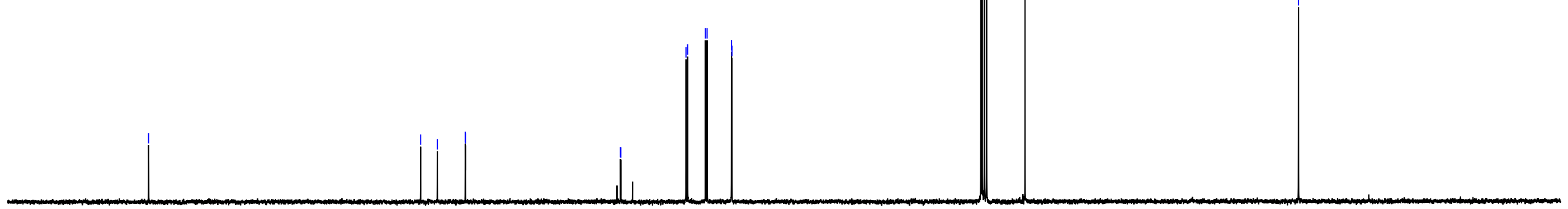

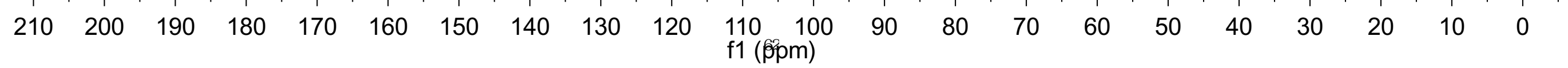


<smiles>CC(C)(O)c1cc(F)ccc1OC1COC1</smiles>

1c

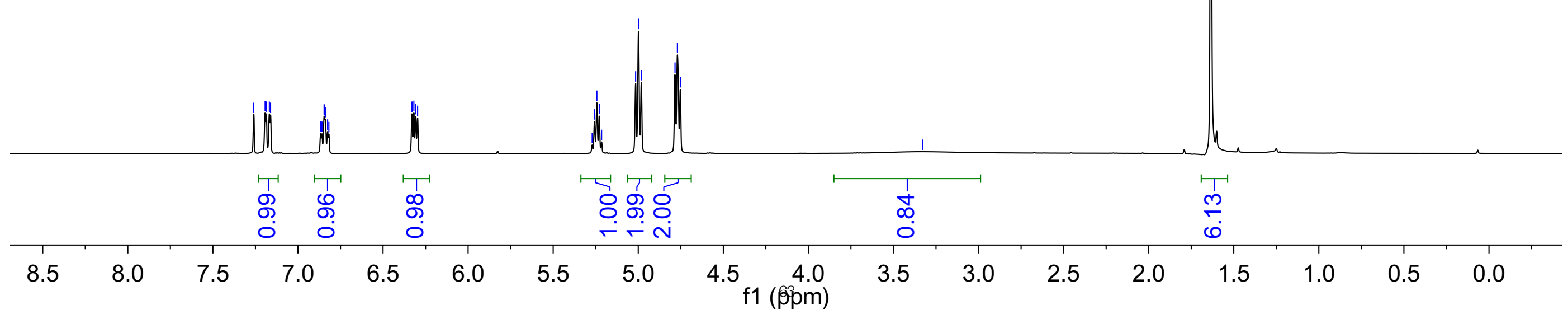


<smiles>CC(C)(O)c1cc(F)ccc1OC1COC1</smiles>

$1 c$

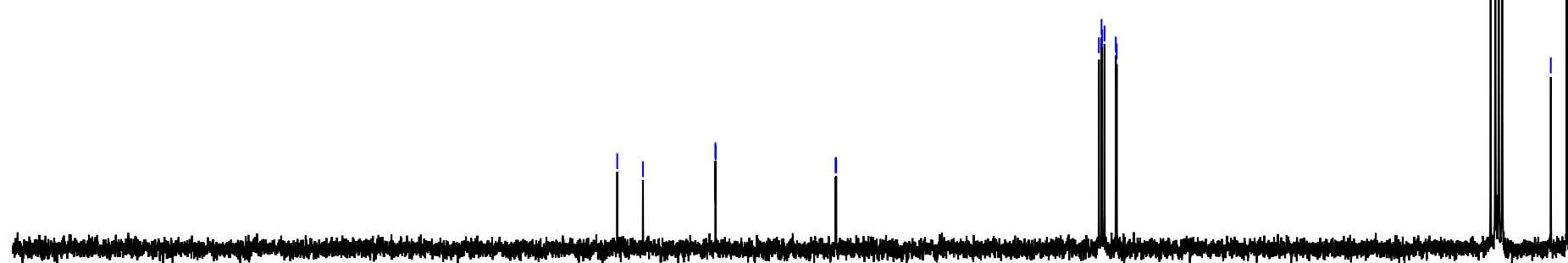


<smiles>CC(=O)c1cc(Cl)ccc1OC1COC1</smiles>

S4

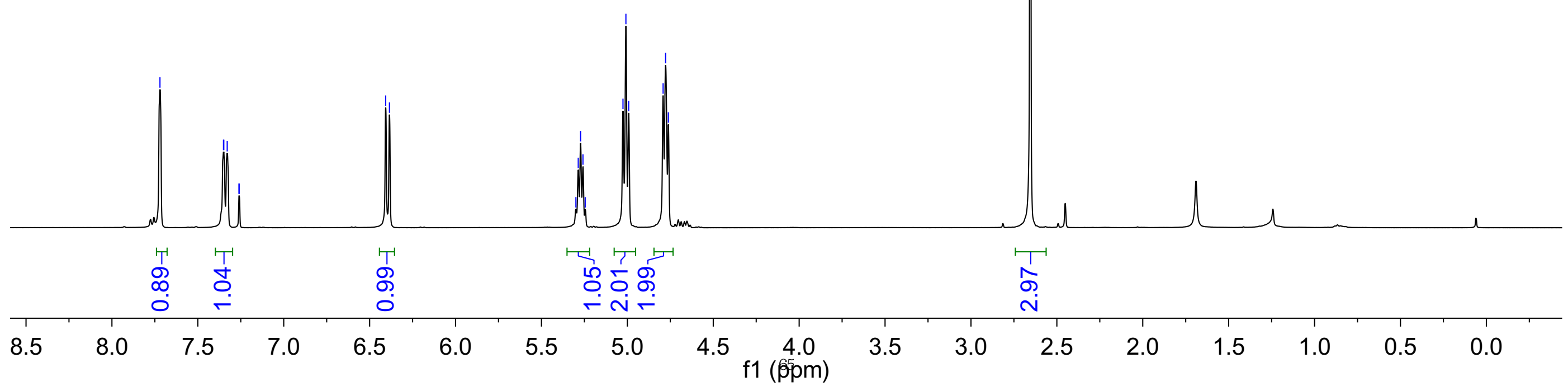


<smiles>CC(=O)c1cc(Cl)ccc1OC1COC1</smiles>

S4

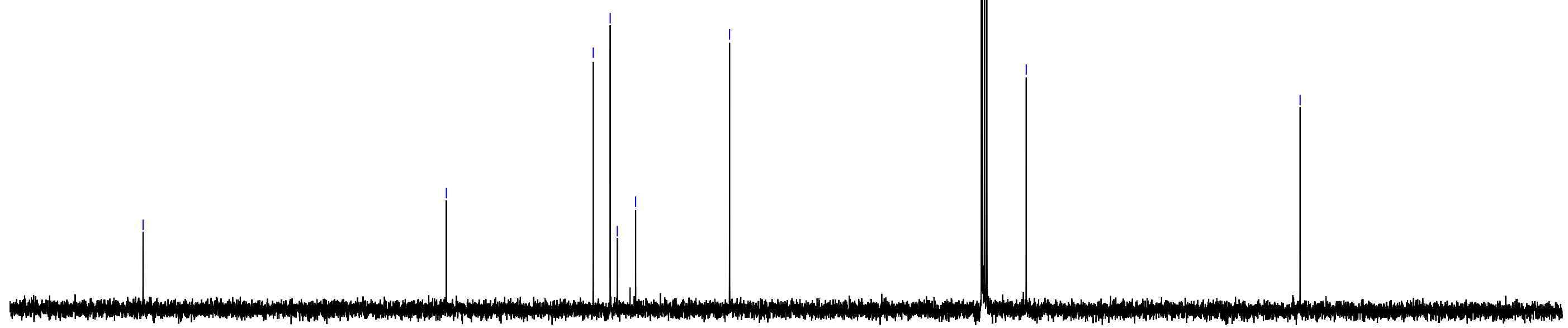

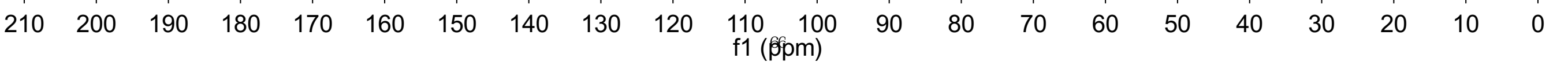




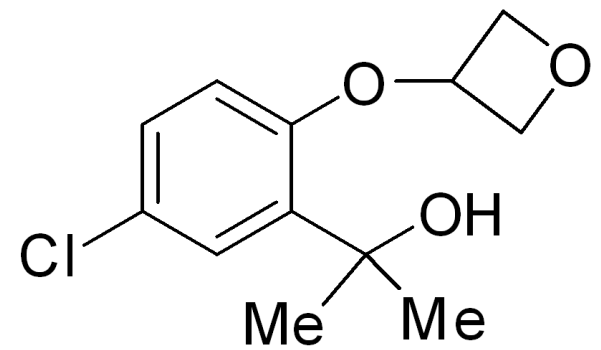

1d

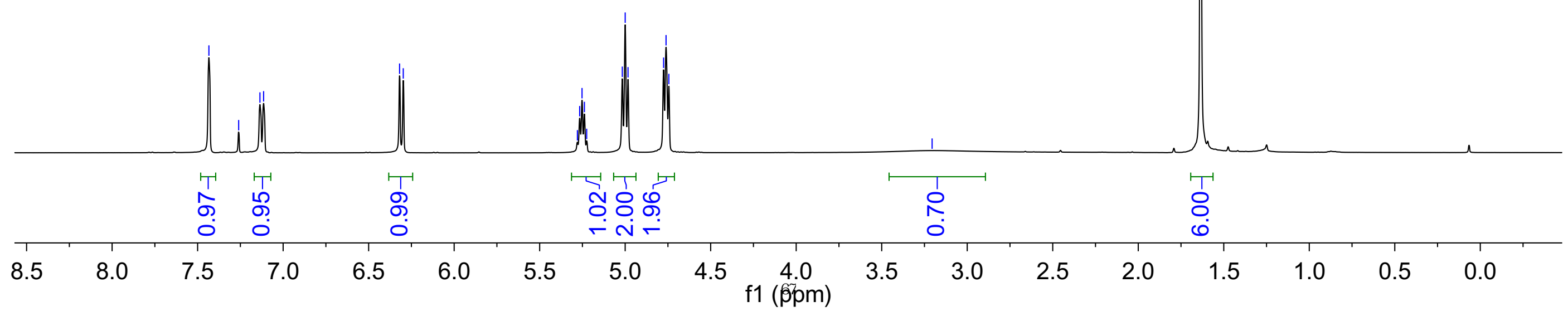


<smiles>CC(C)(O)c1cc(Cl)ccc1OC1COC1</smiles>

$1 d$

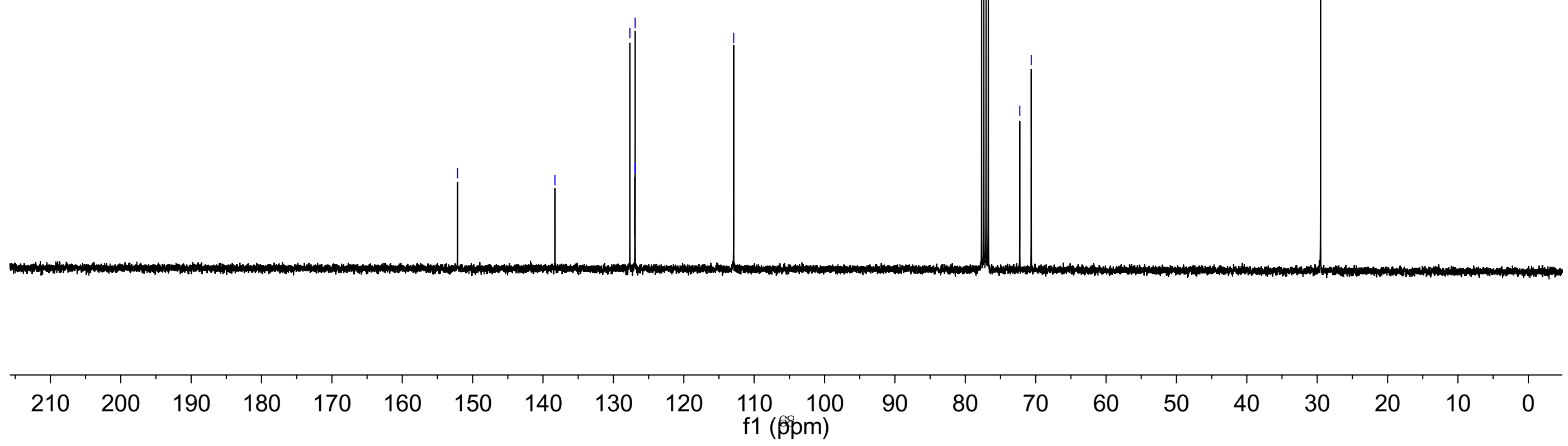


<smiles>CC(=O)c1cc(Br)ccc1OC1COC1</smiles>

S5

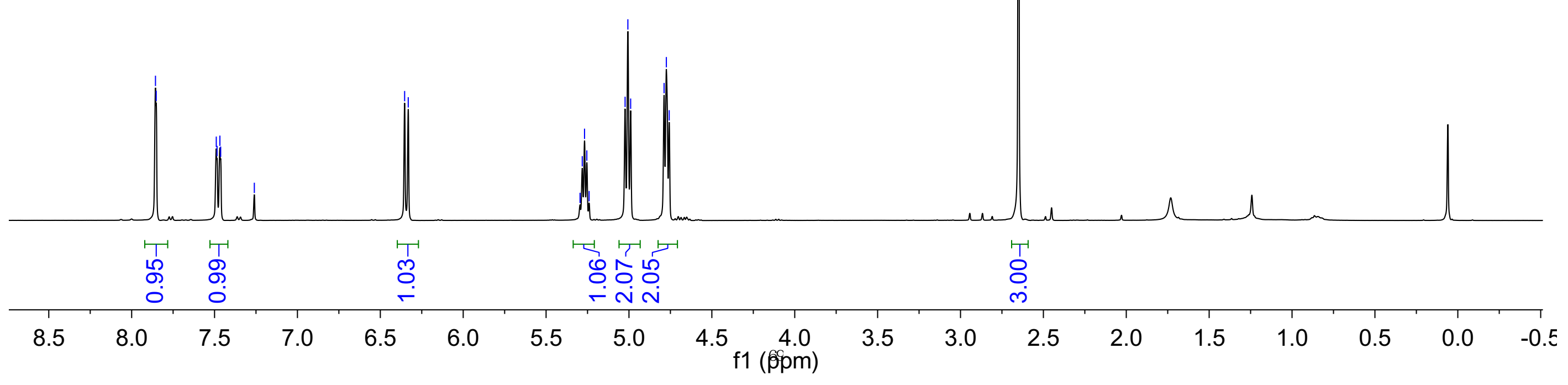




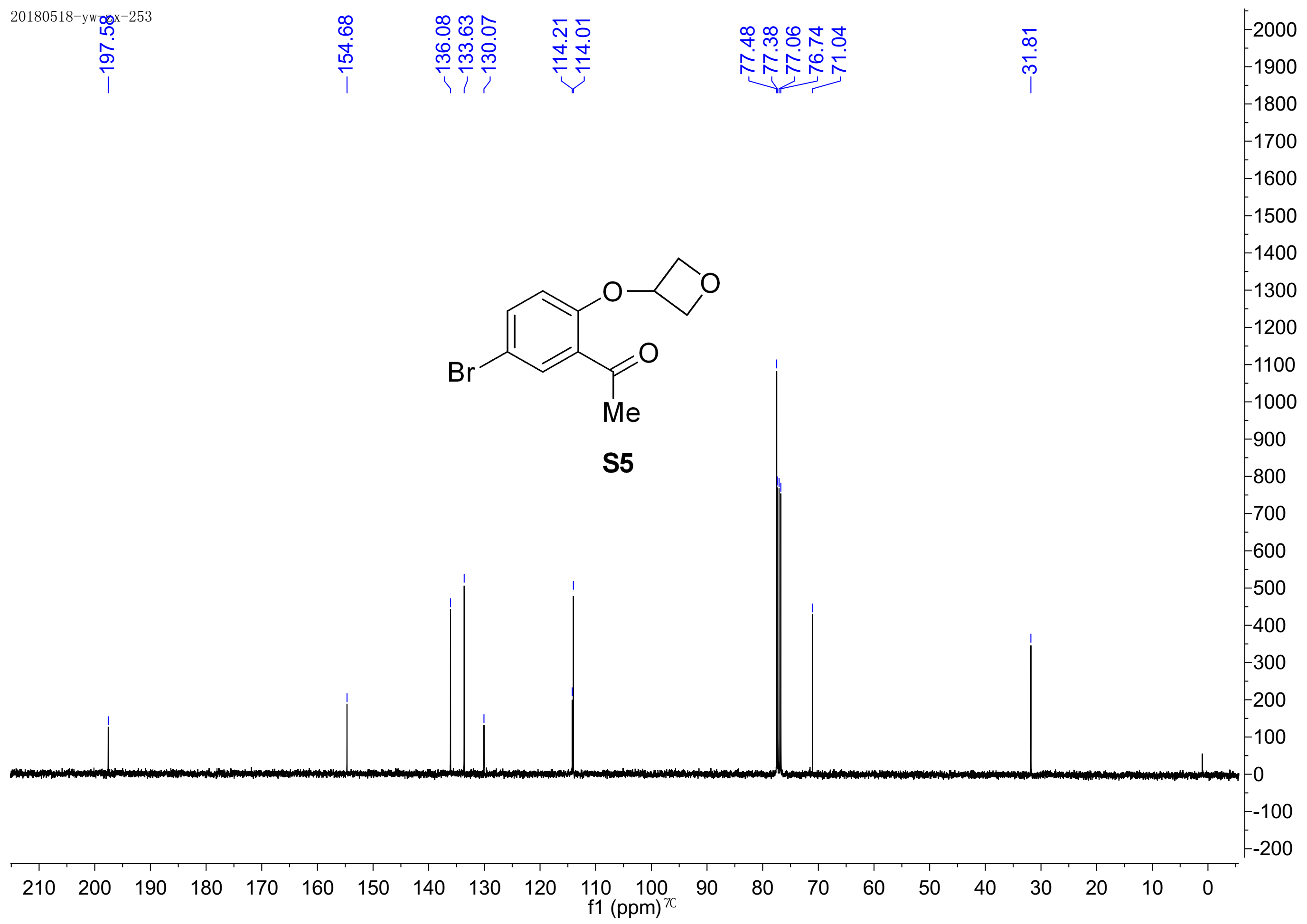


<smiles>CC(C)(O)c1cc(Br)ccc1OC1COC1</smiles>

$1 e$

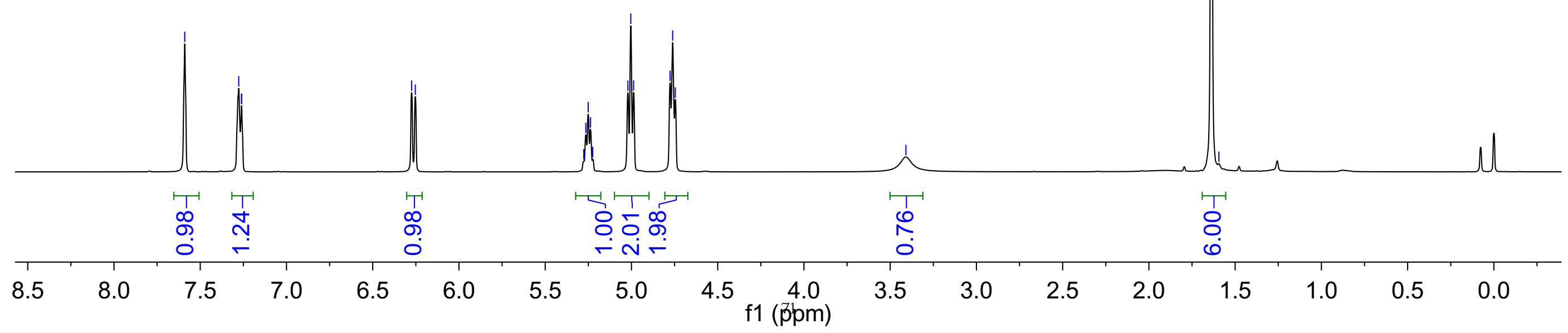




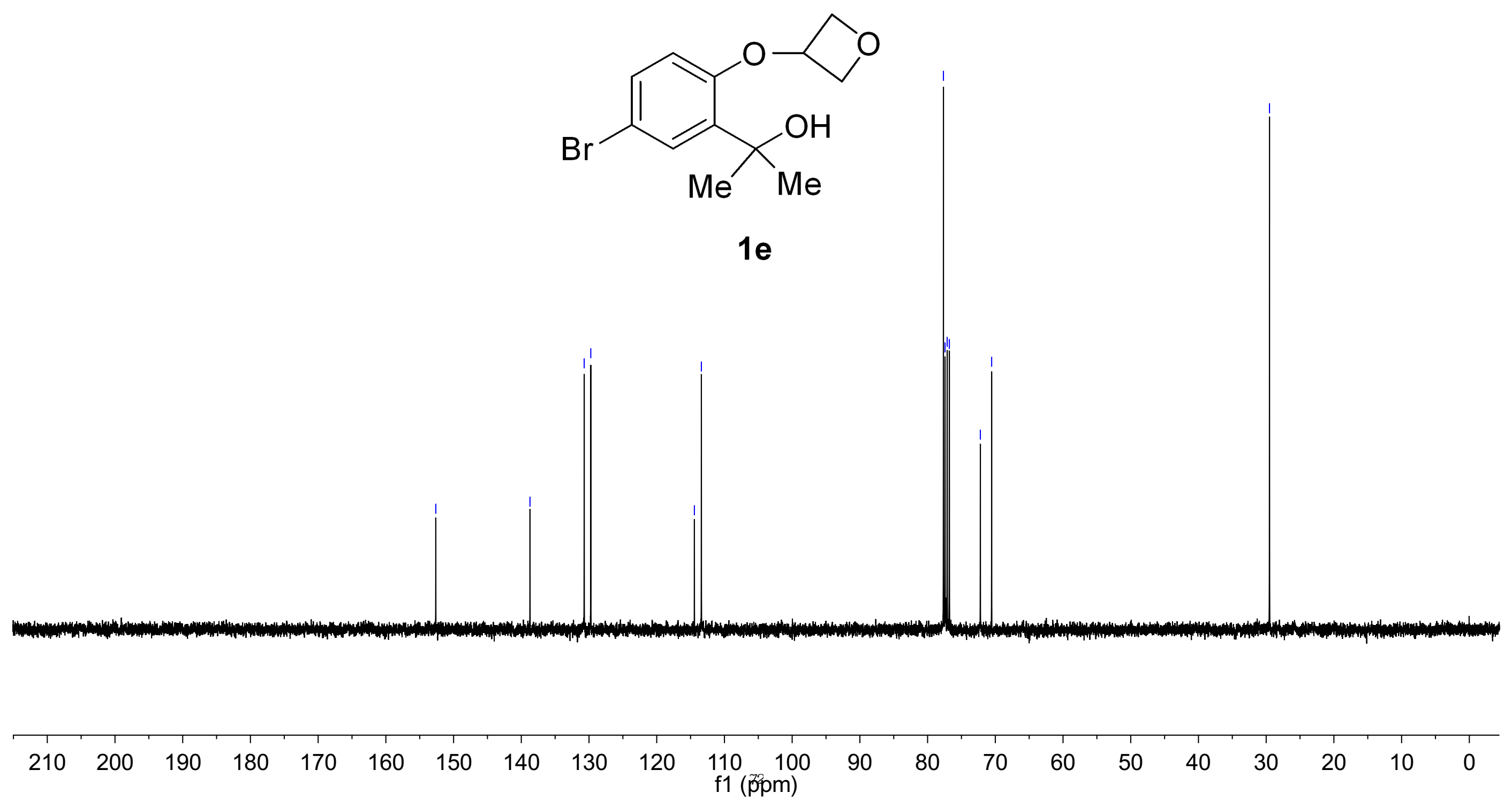




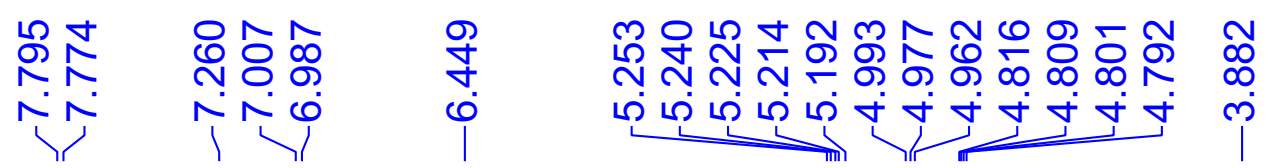

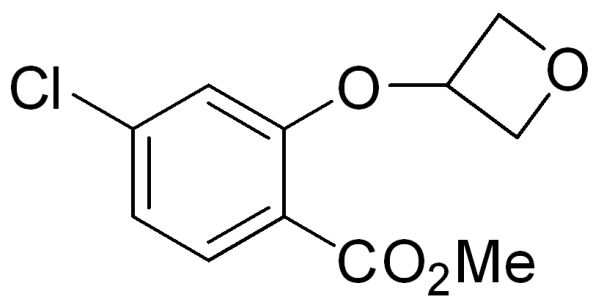

s9

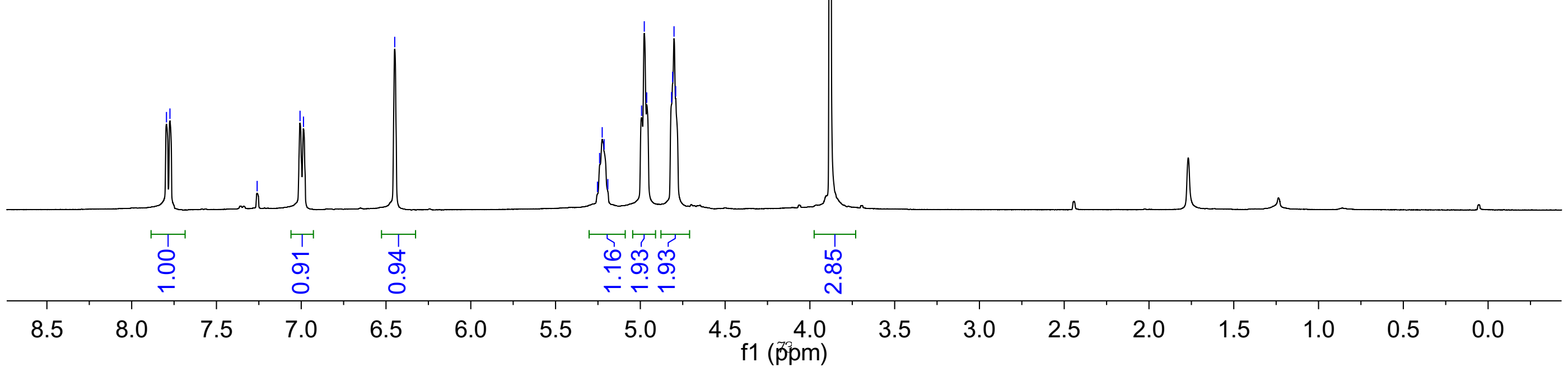




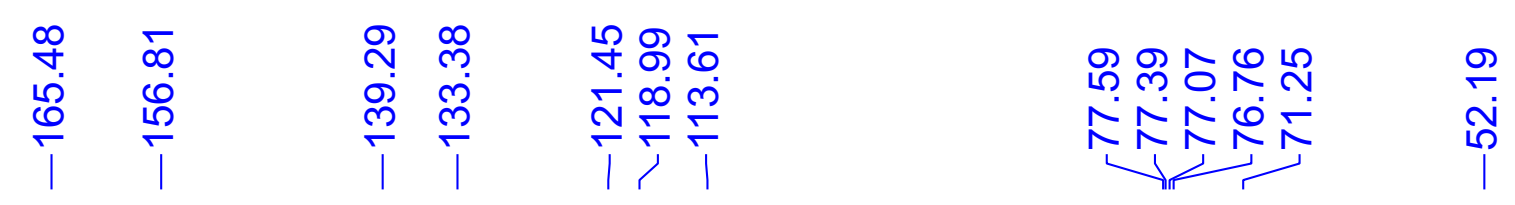<smiles>COC(=O)c1ccc(Cl)cc1OC1COC1</smiles>

S9

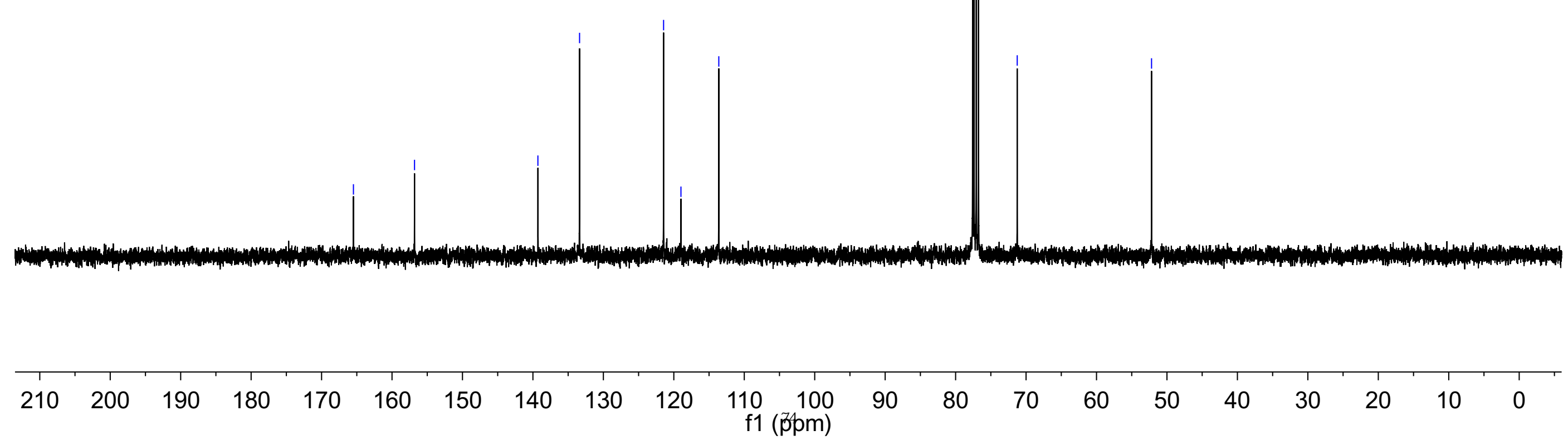



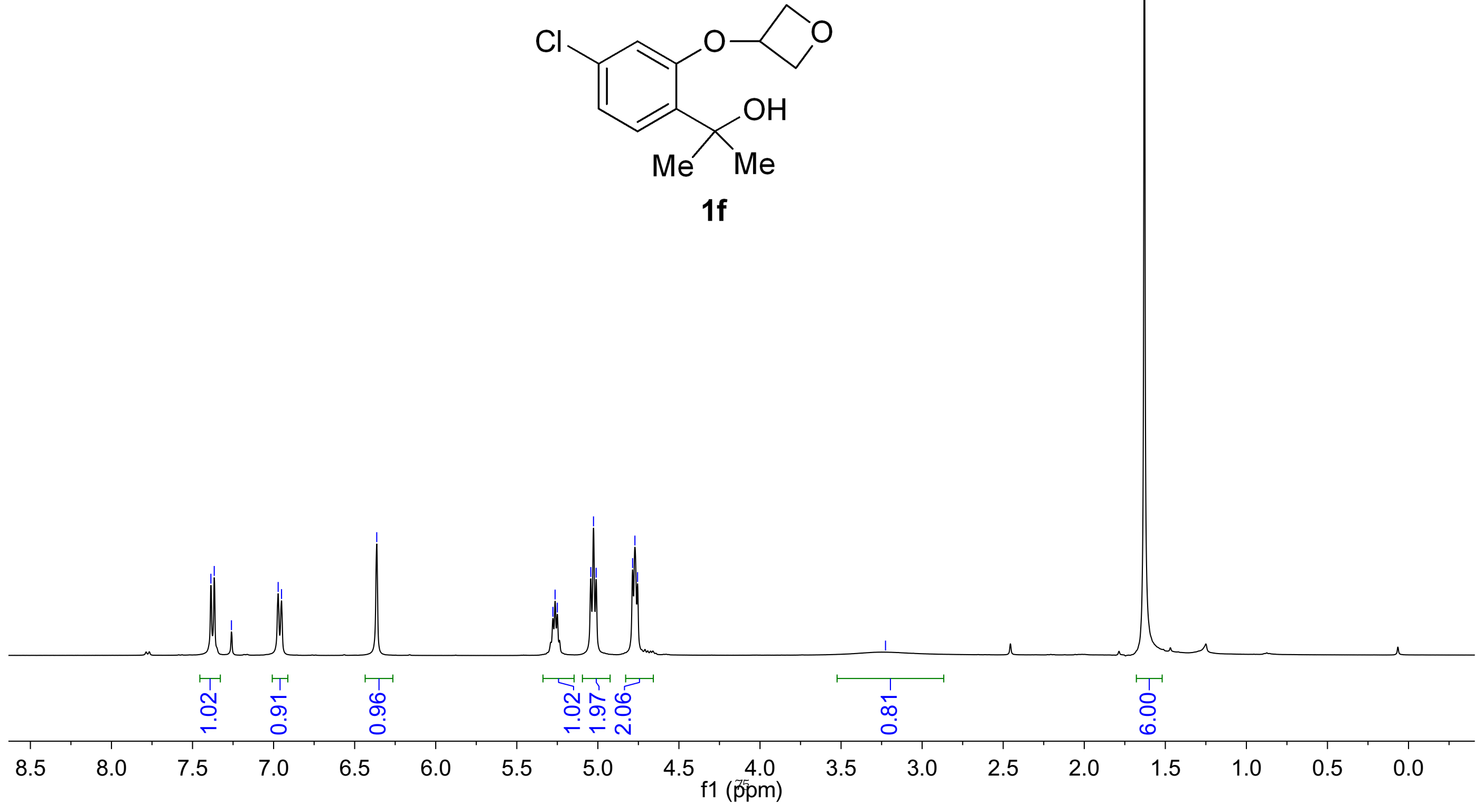
<smiles>CC(C)(O)c1ccc(Cl)cc1OC1COC1</smiles>

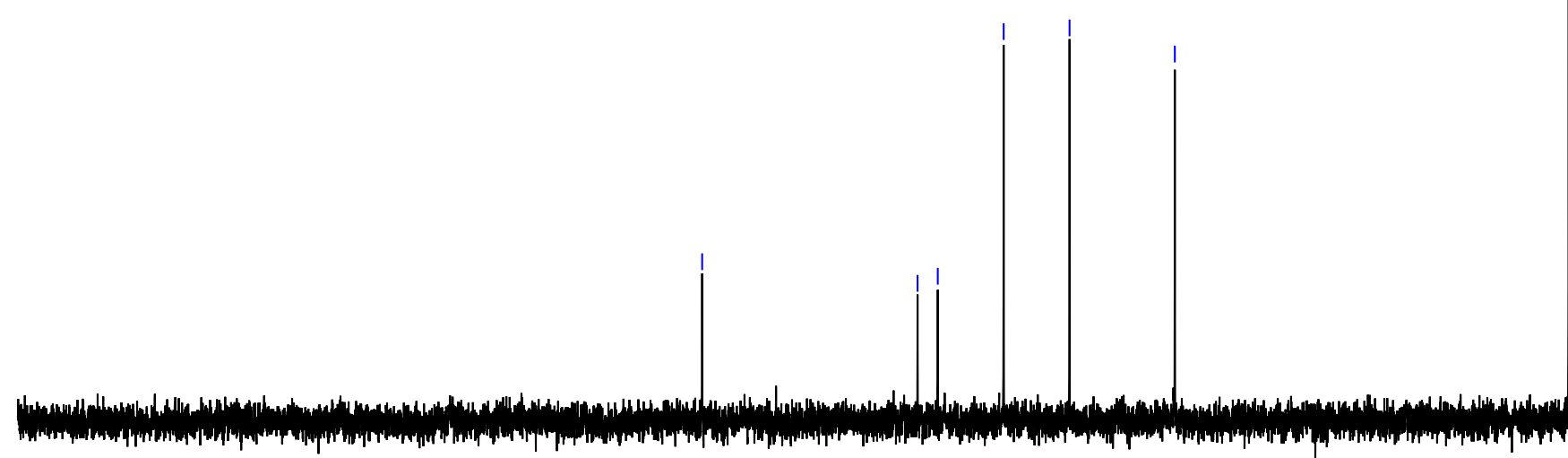




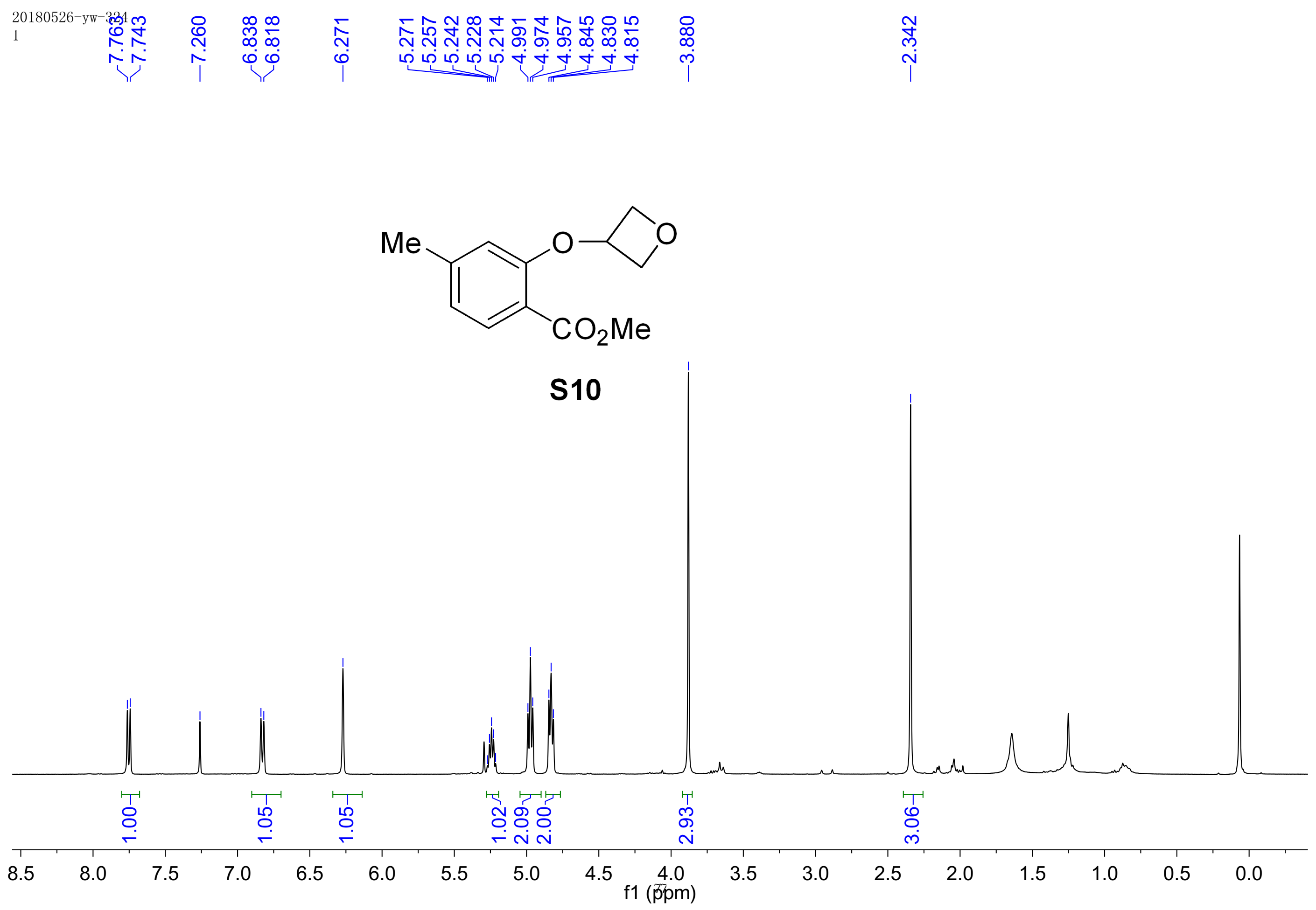


<smiles>COC(=O)c1ccc(C)cc1OC1COC1</smiles>

$\mathrm{S} 10$ 


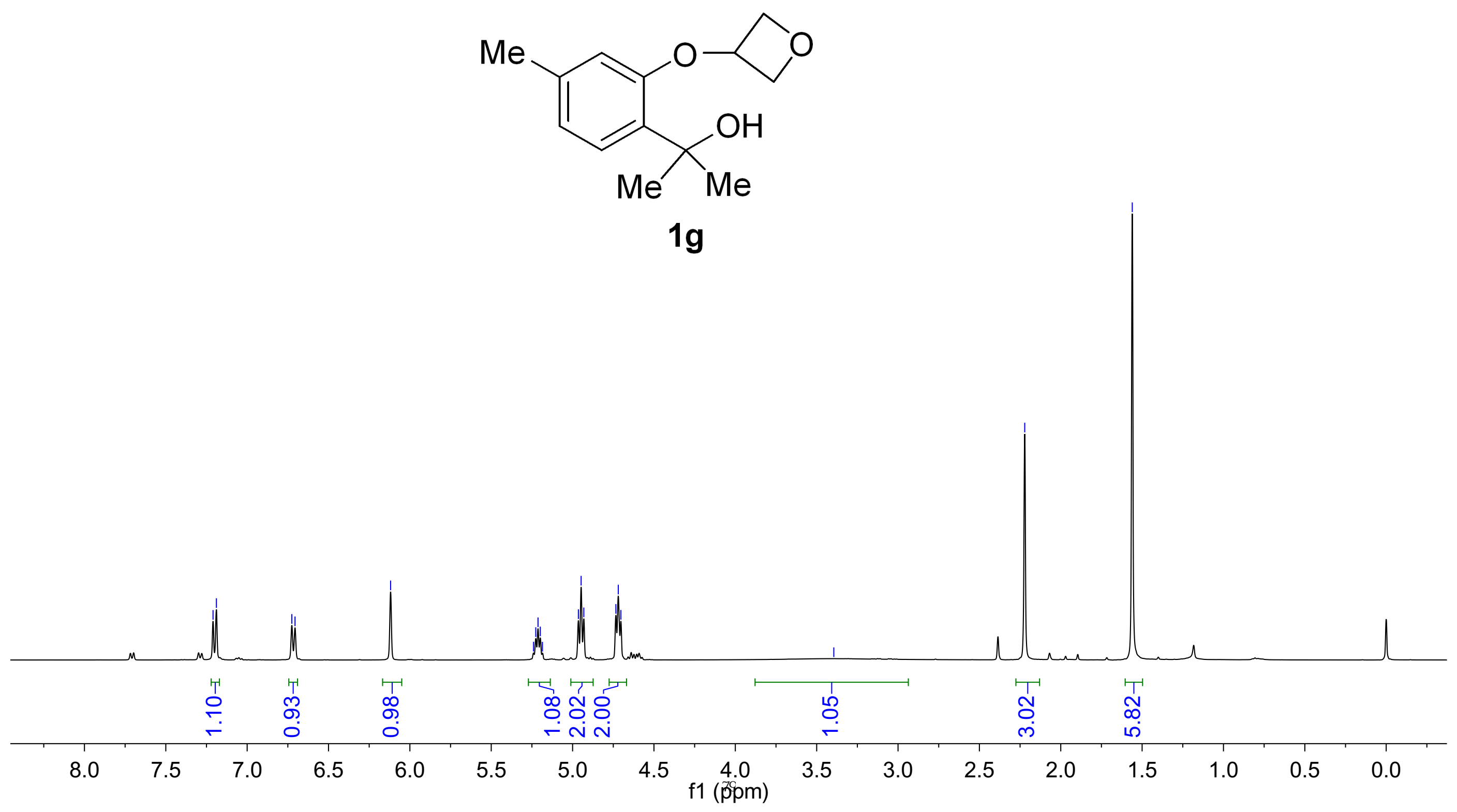




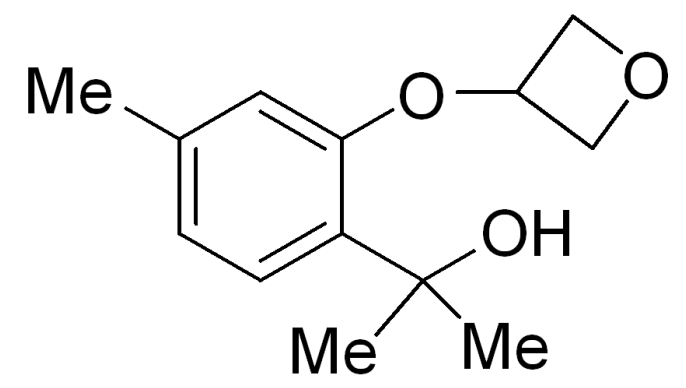

$1 \mathrm{~g}$

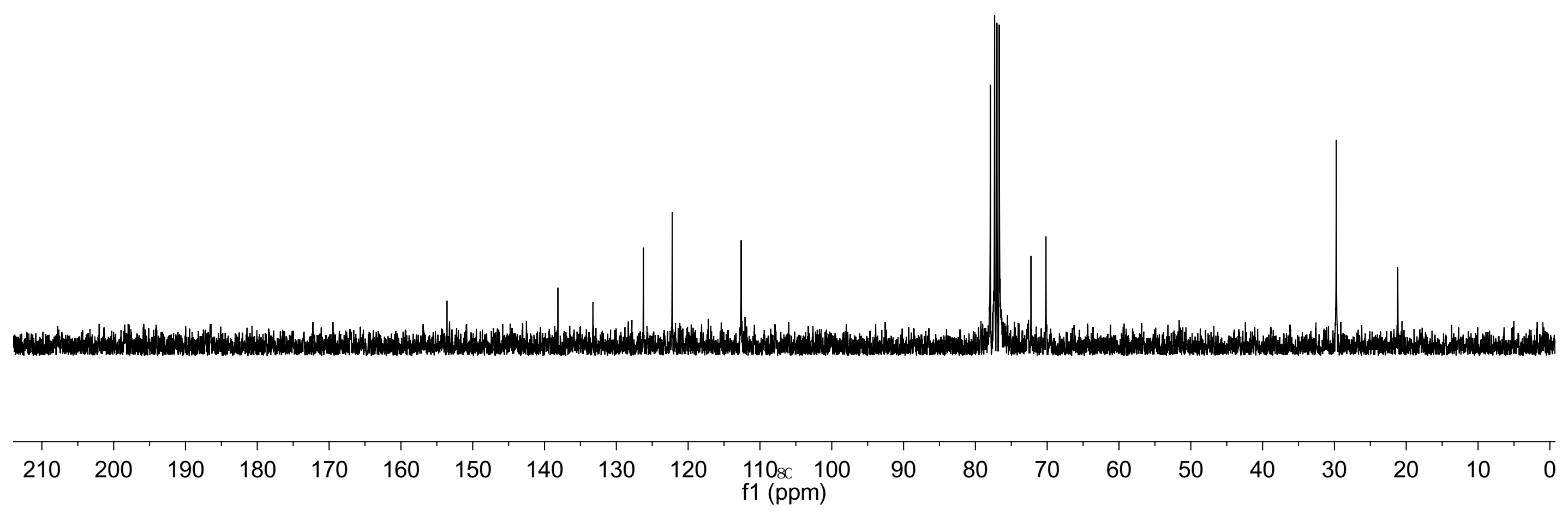




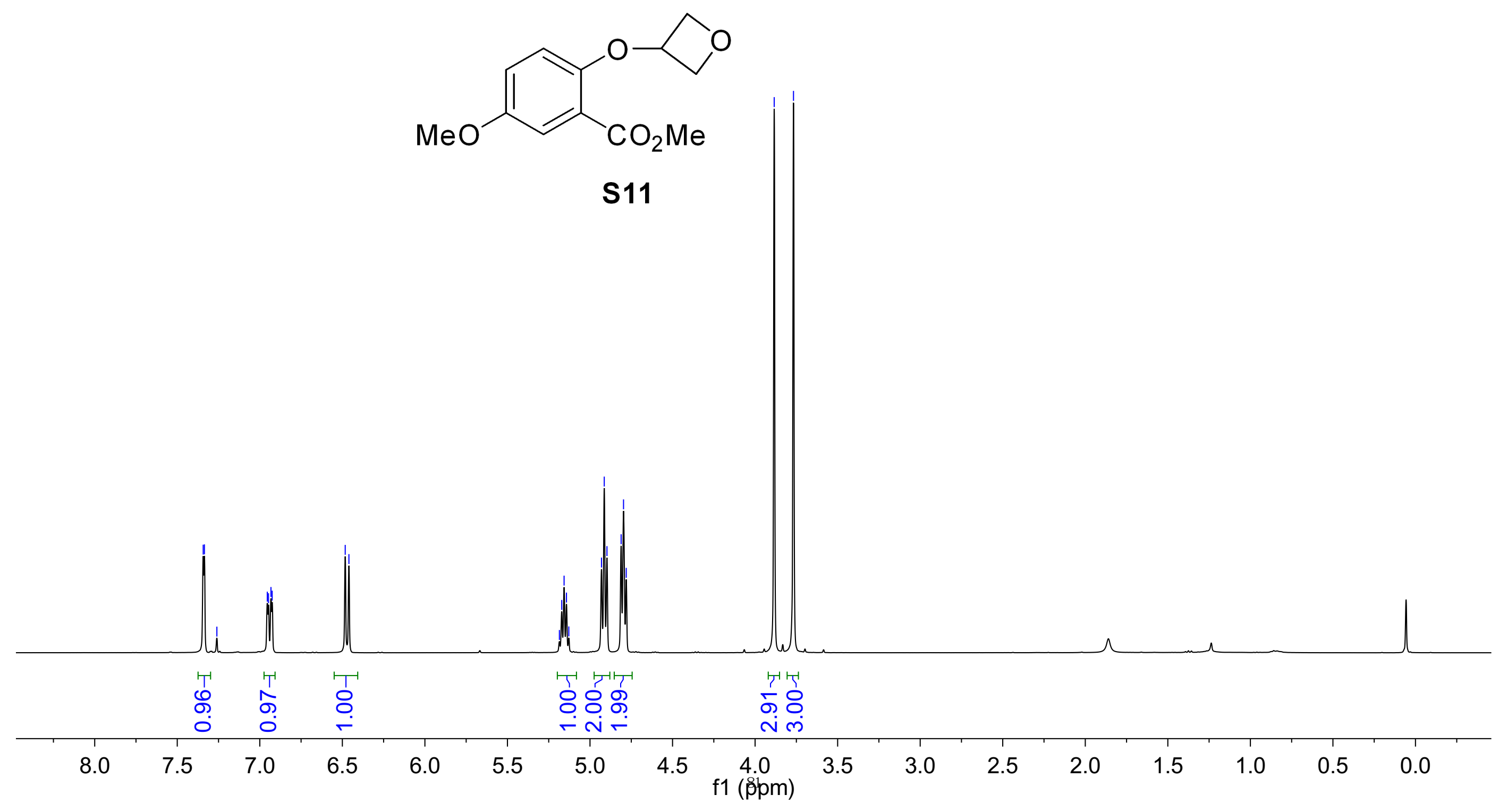


<smiles>COC(=O)c1cc(OC)ccc1OC1COC1</smiles>

S11

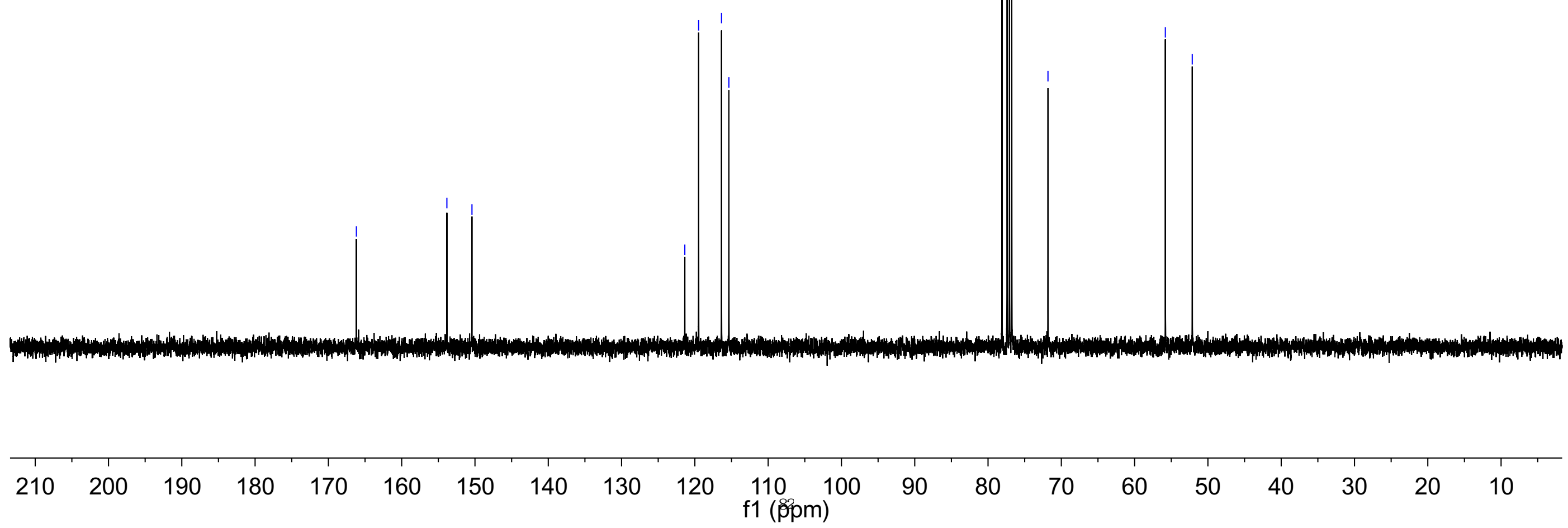




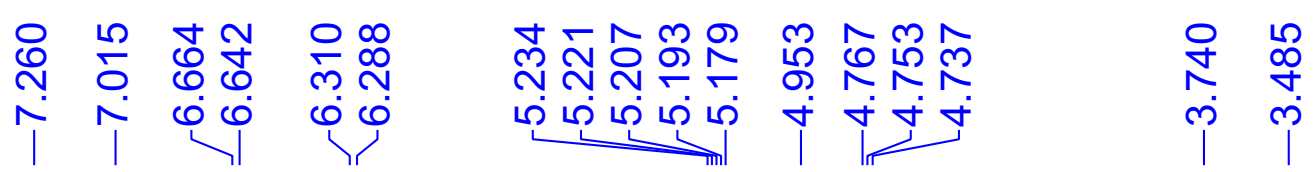

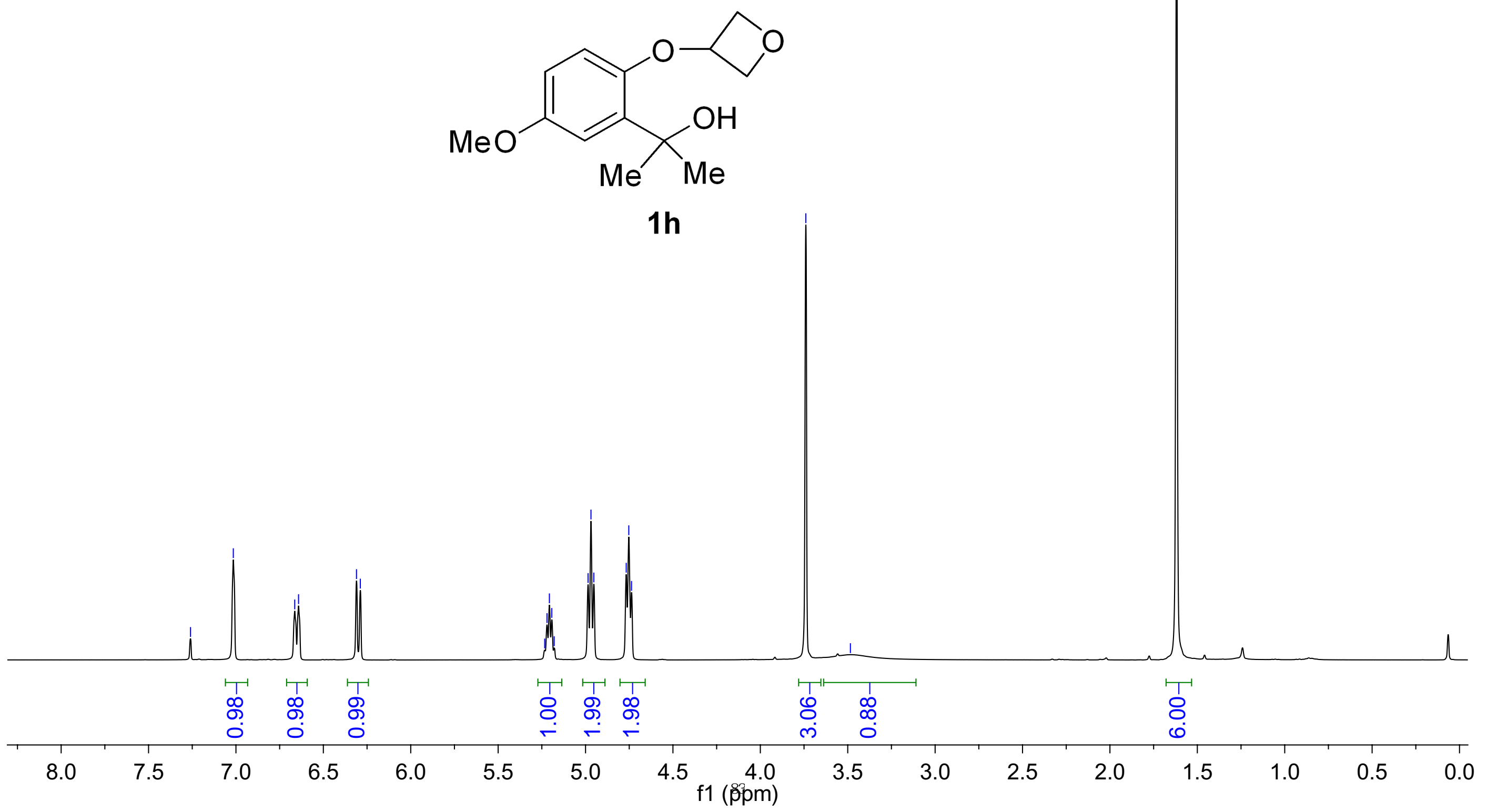




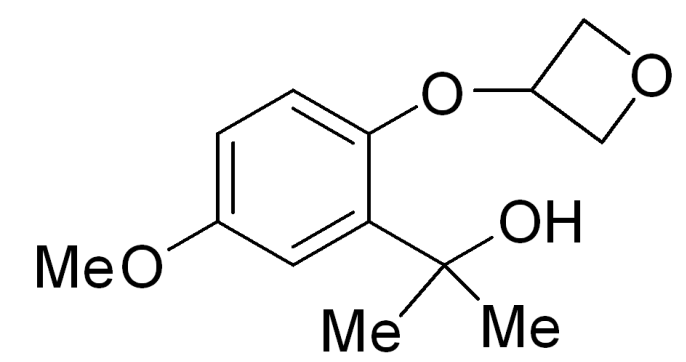

1h

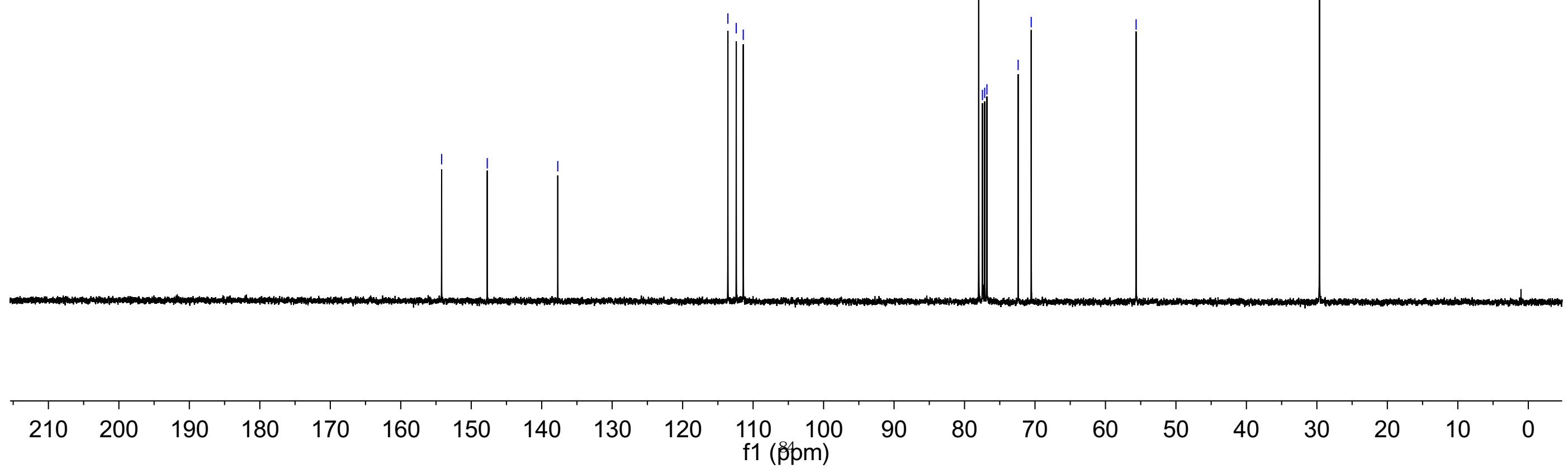




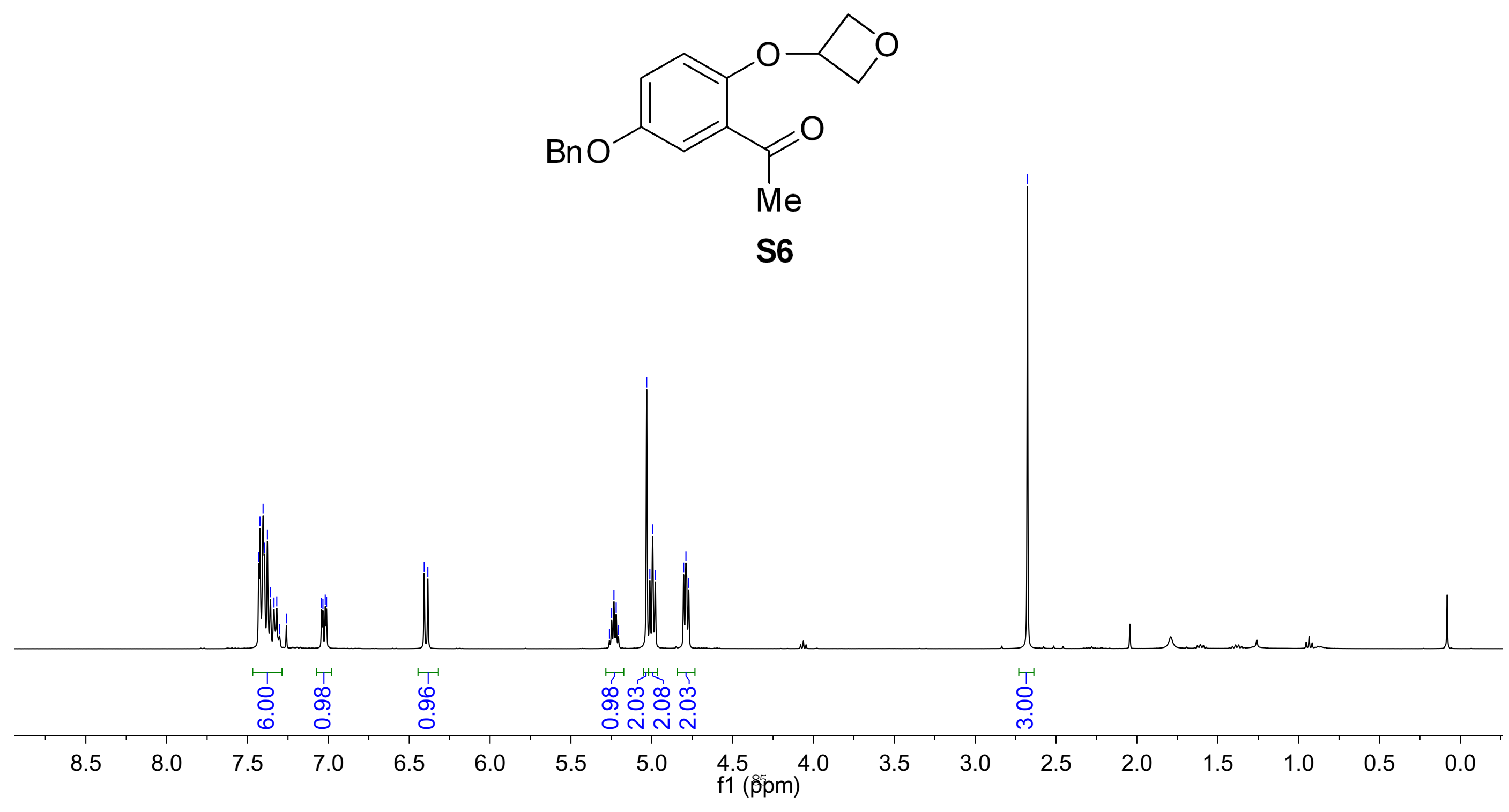




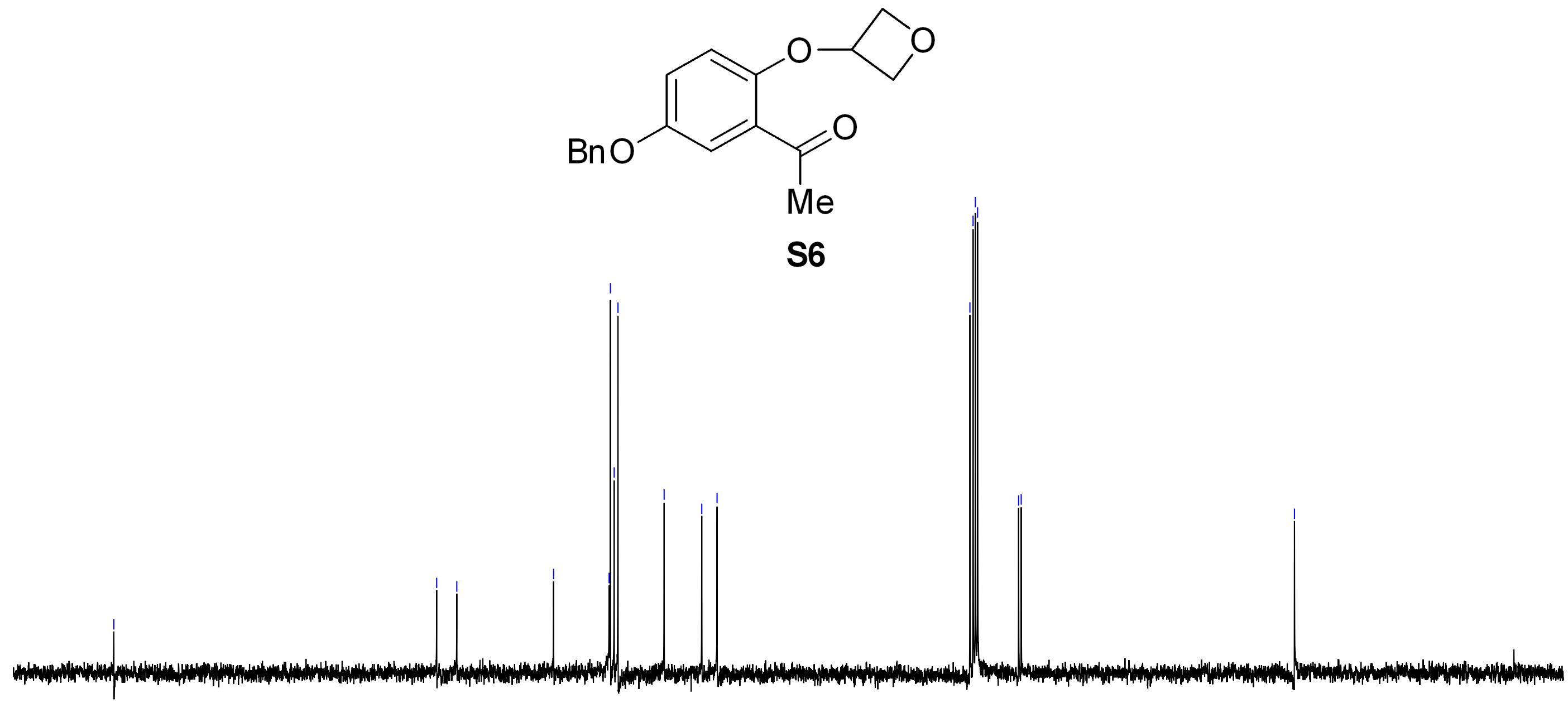

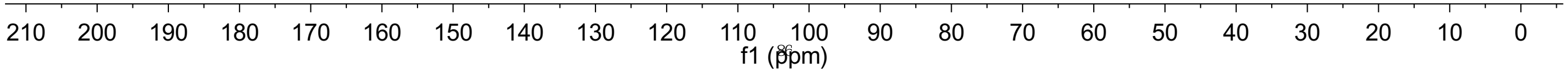



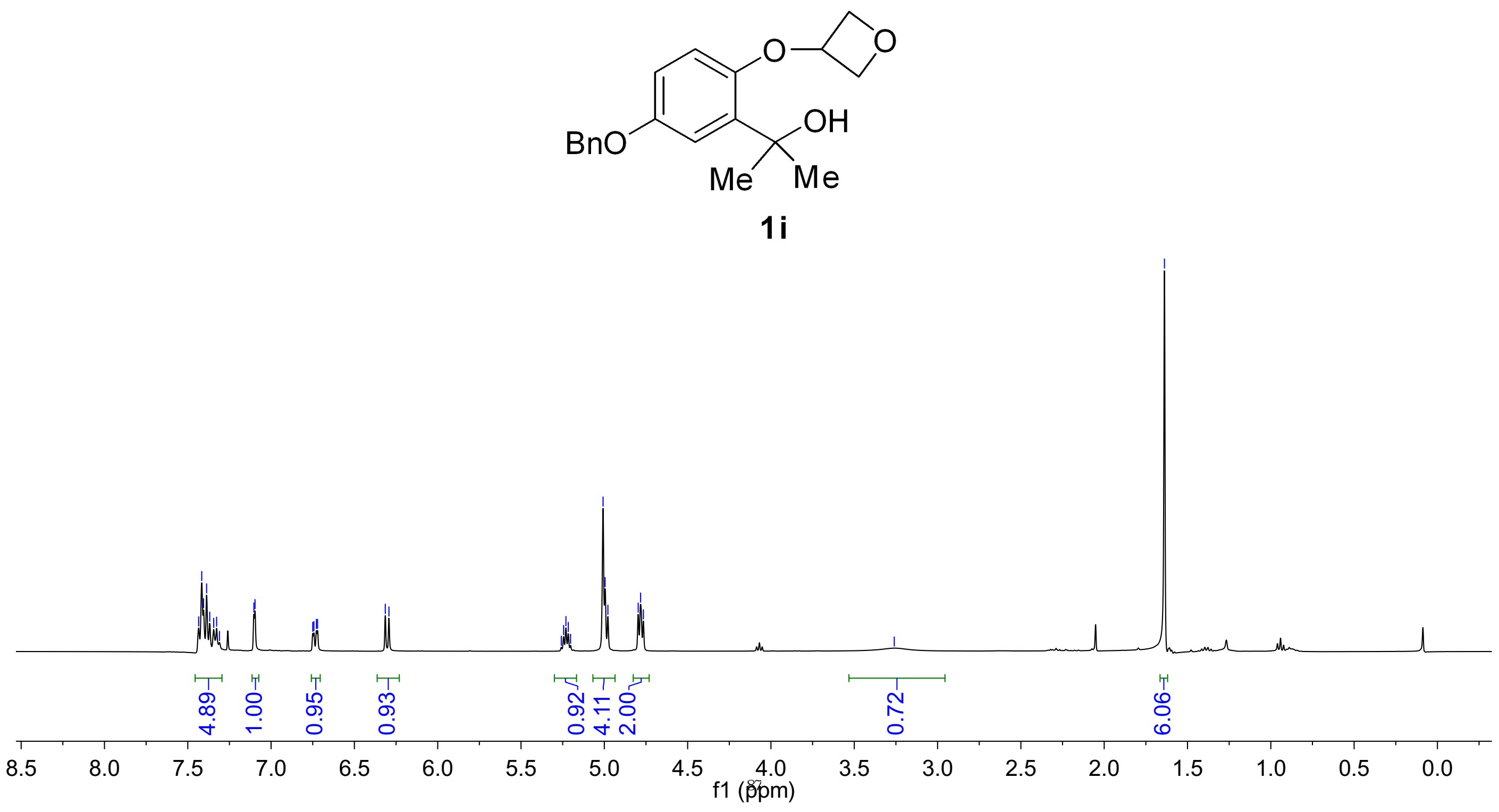
<smiles>CC(C)(O)c1cc(OCc2ccccc2)ccc1OC1COC1</smiles>

$1 \mathbf{i}$

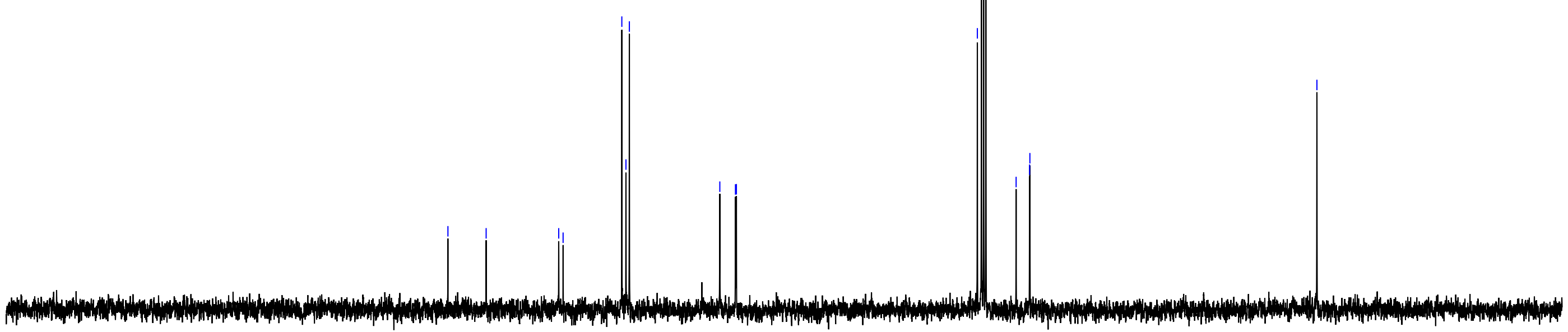




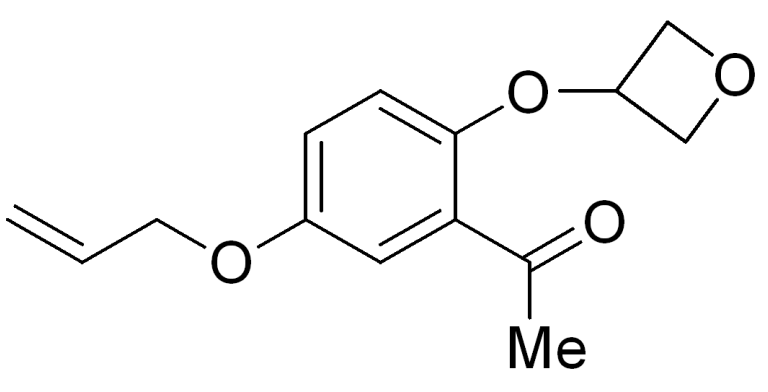

S7

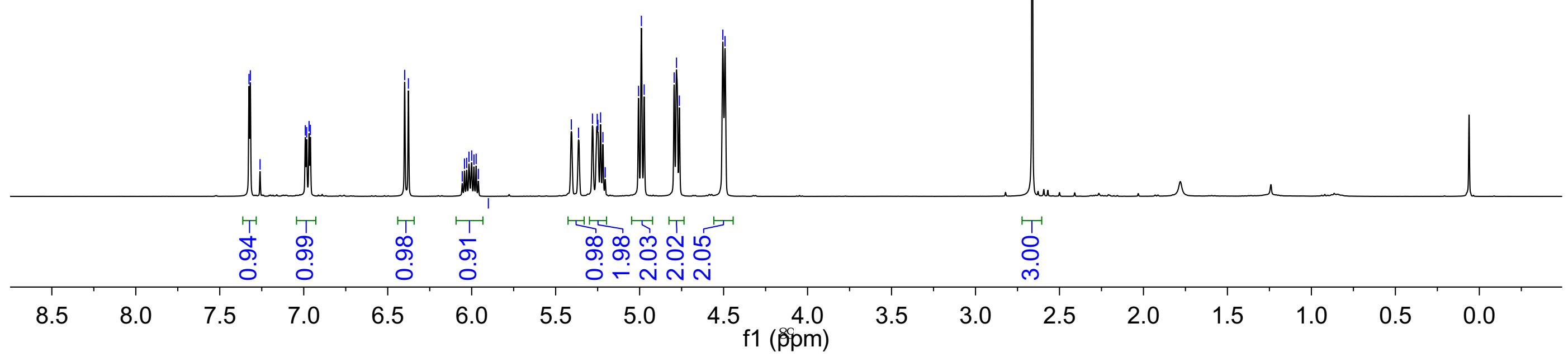


<smiles>C=CCOc1ccc(OC2COC2)c(C(C)=O)c1</smiles>

S7

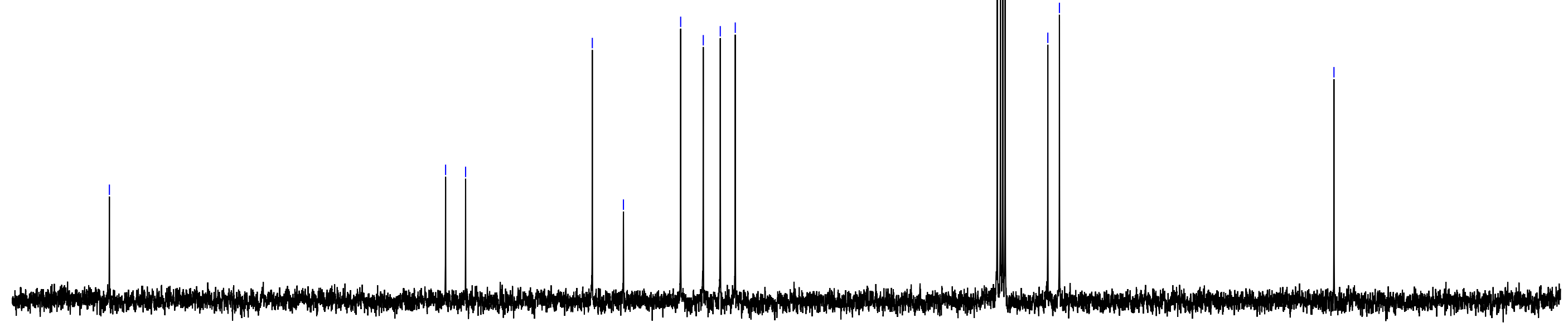

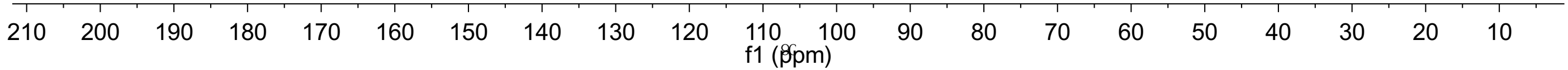




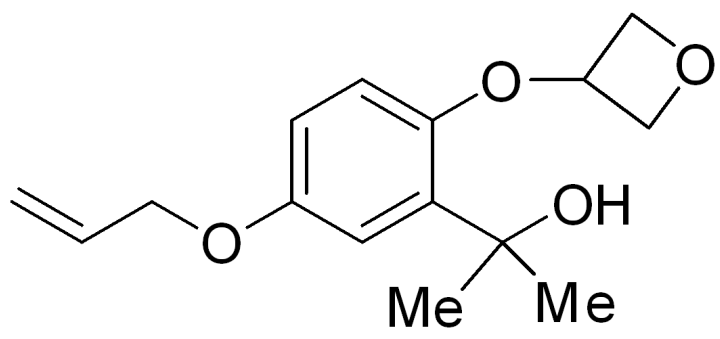

$1 \mathbf{j}$

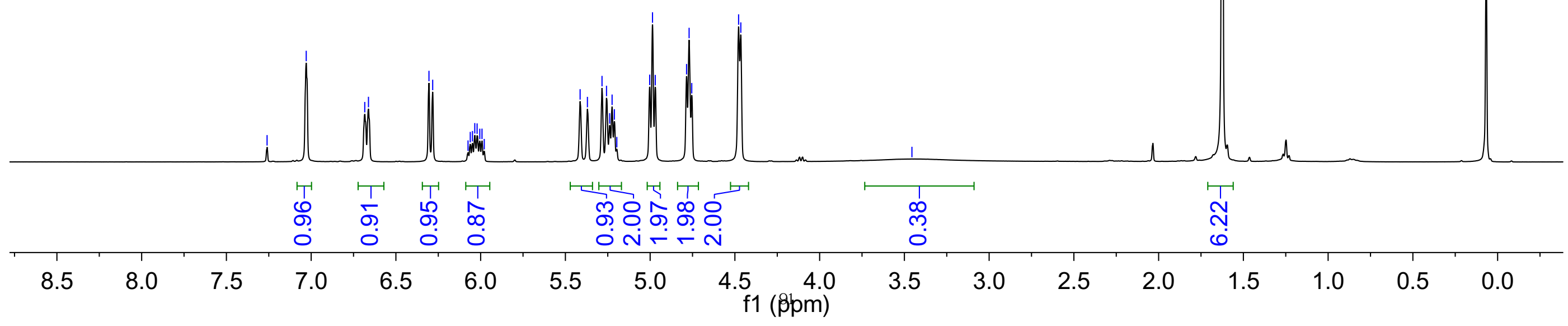




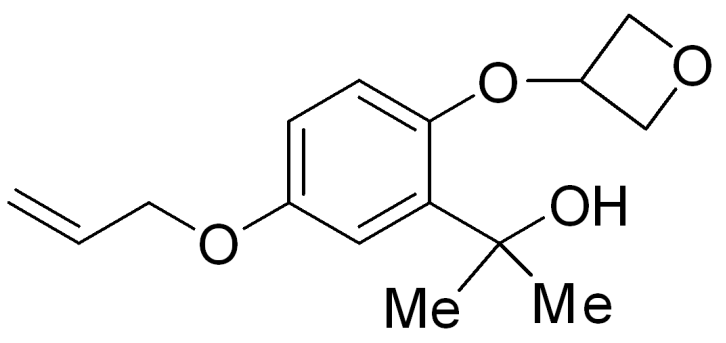

1j

\begin{tabular}{lllllllllllllllllllllll}
\hline 210 & 200 & 190 & 180 & 170 & 160 & 150 & 140 & 130 & 120 & $\begin{array}{c}110 \\
\mathrm{f} 1(\beta \mathrm{pm})\end{array}$ & 100 & 90 & 80 & 70 & 60 & 50 & 40 & 30 & 20 & 10 &
\end{tabular}




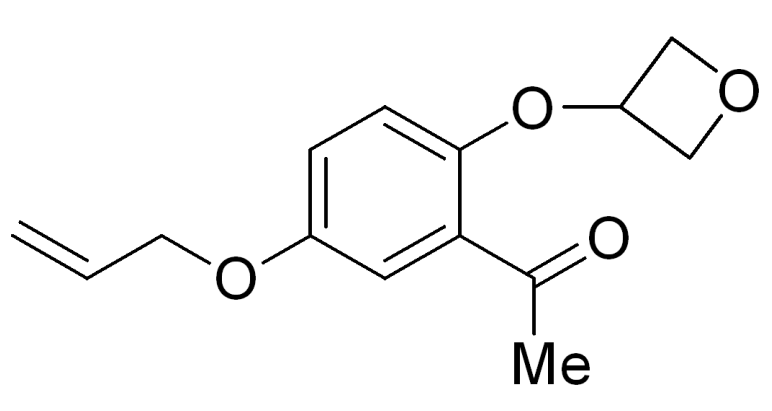

S7

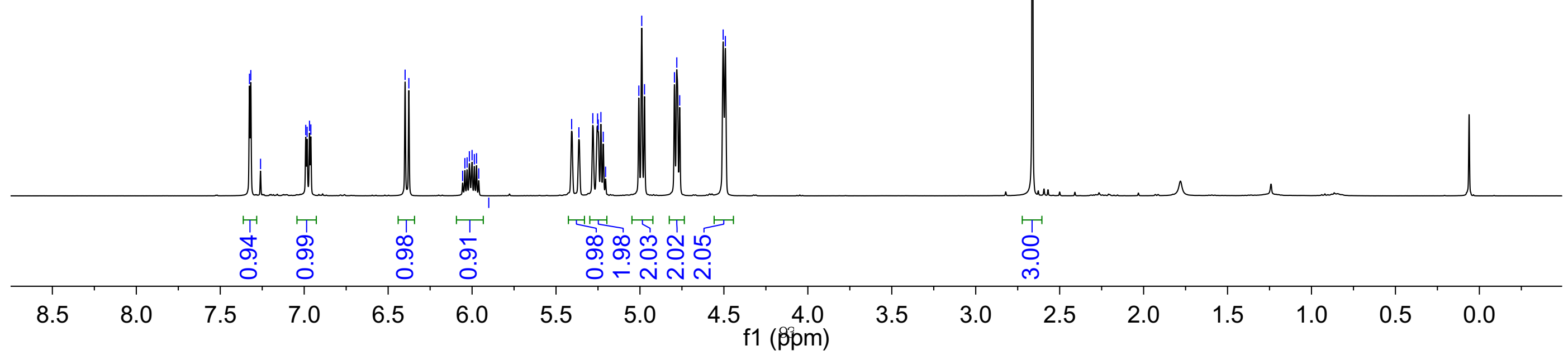




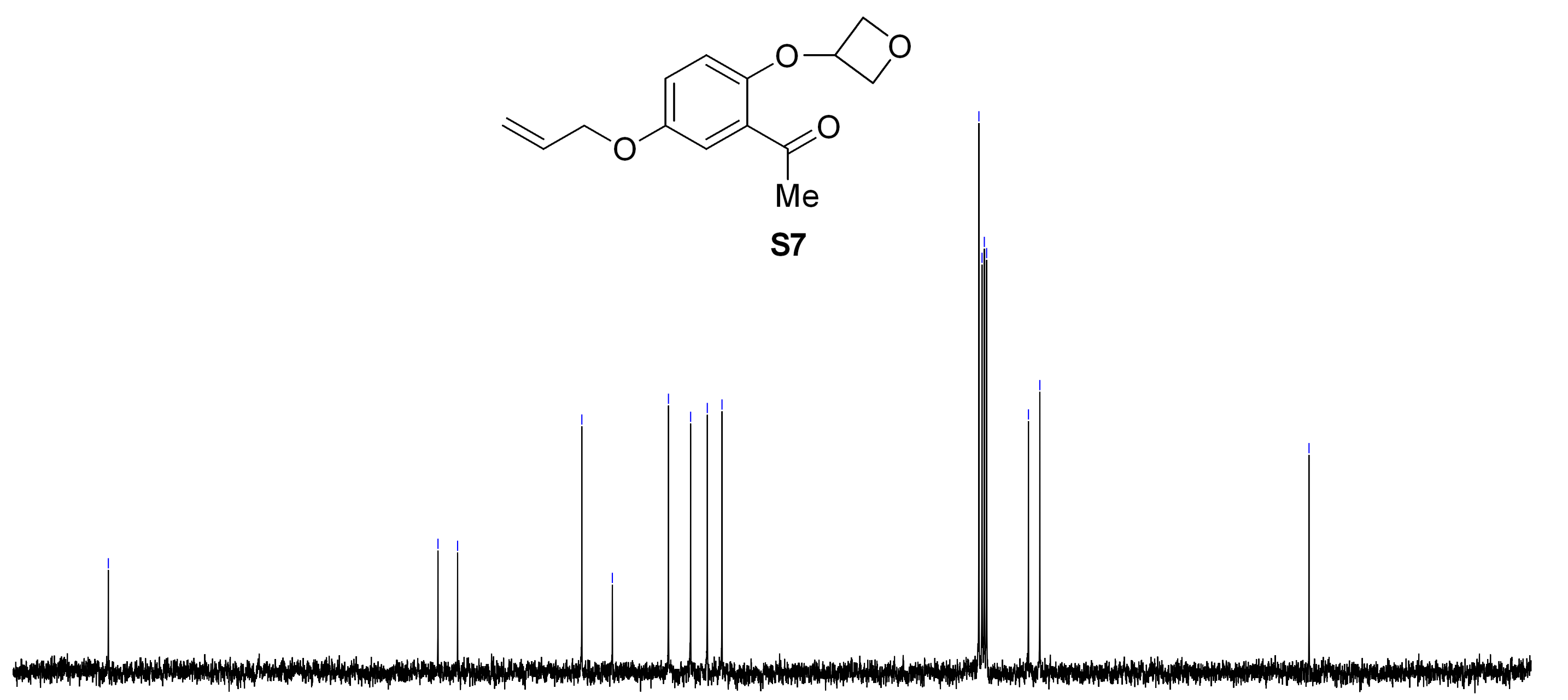

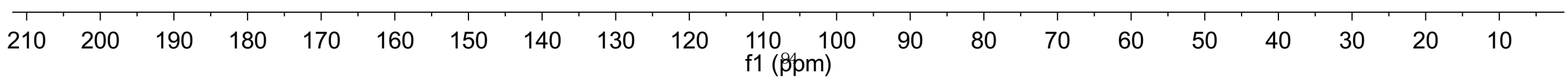




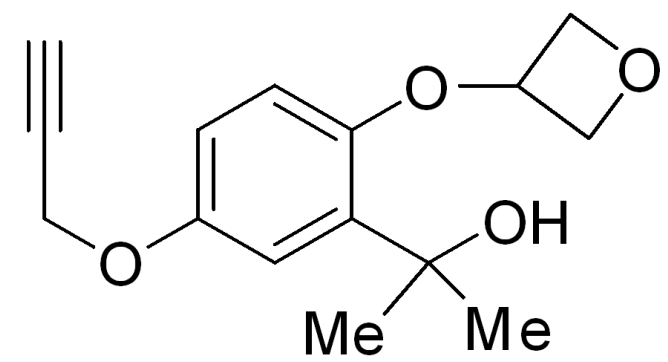

$1 \mathrm{k}$

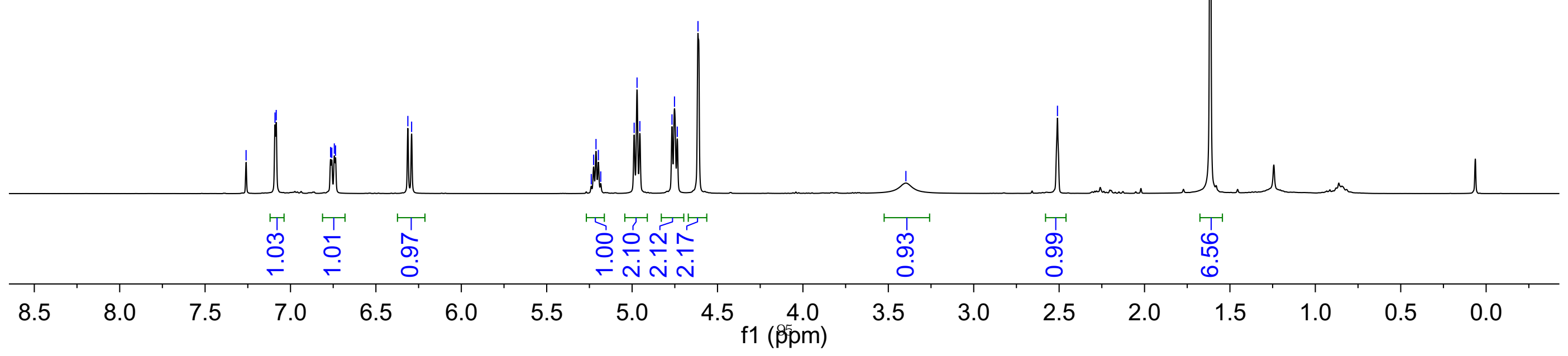




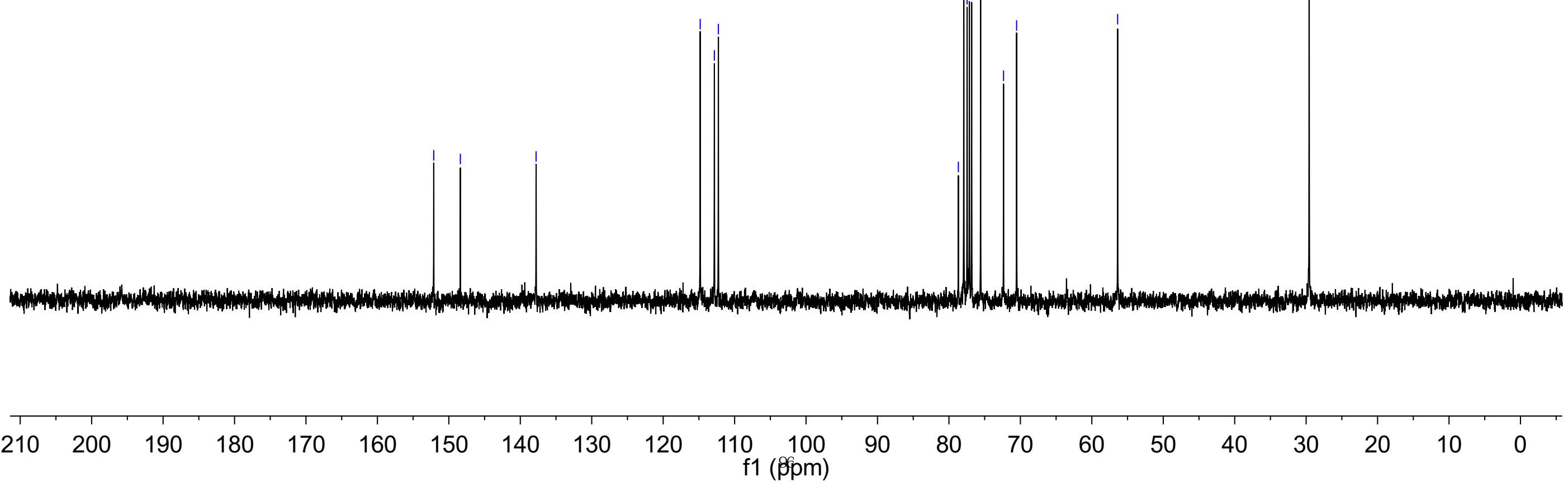<smiles>C#CCOc1ccc(OC2COC2)c(C(C)(C)O)c1</smiles>

$1 \mathrm{k}$ 

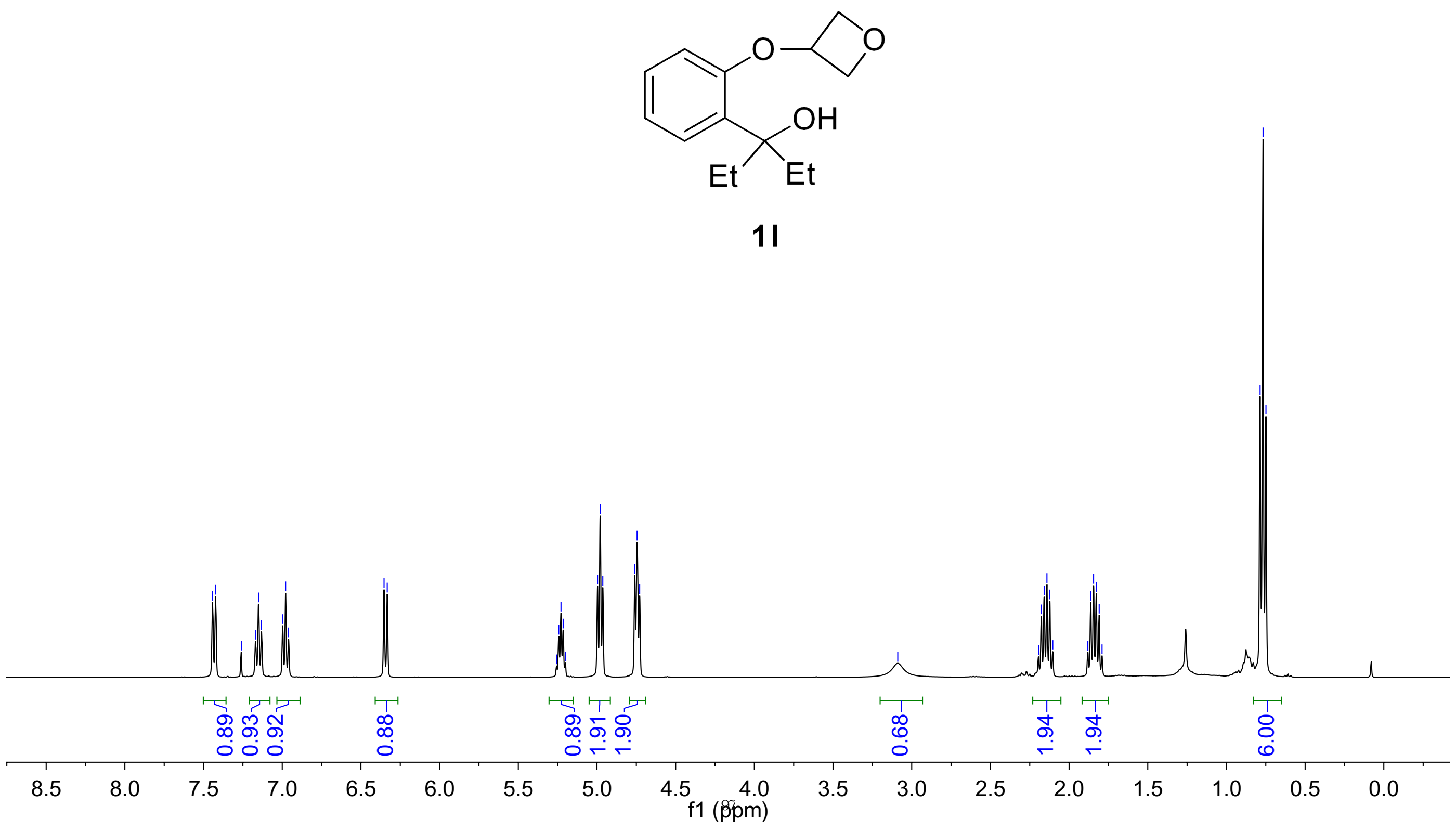


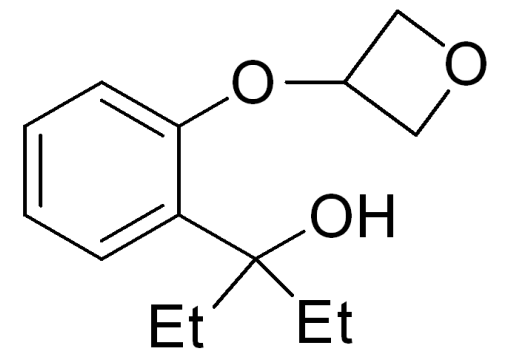

1I

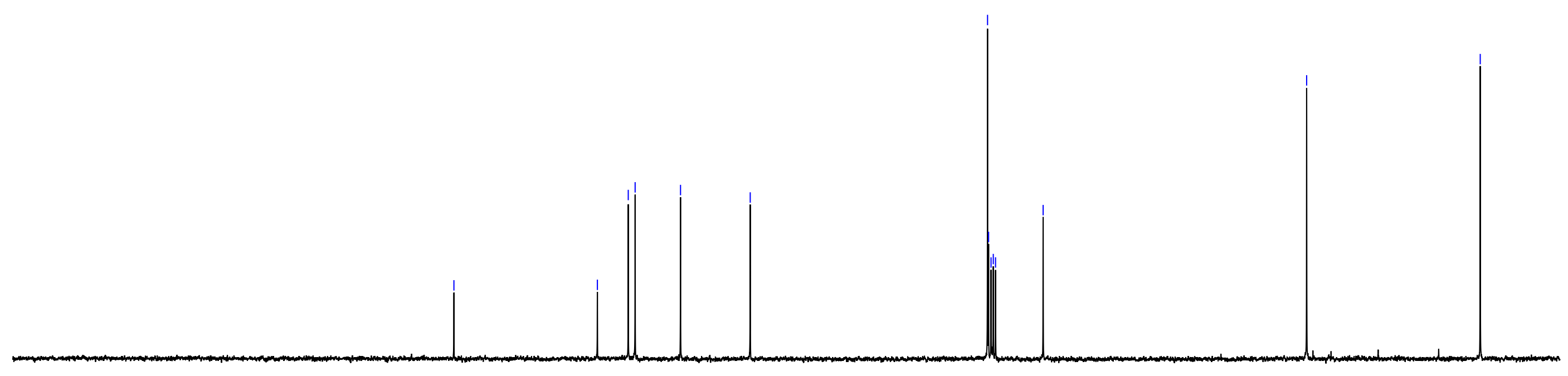

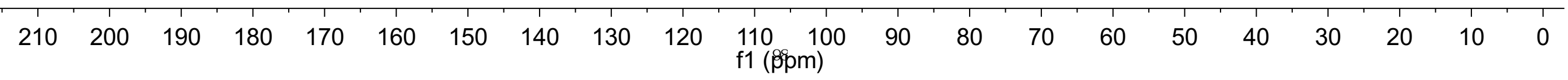



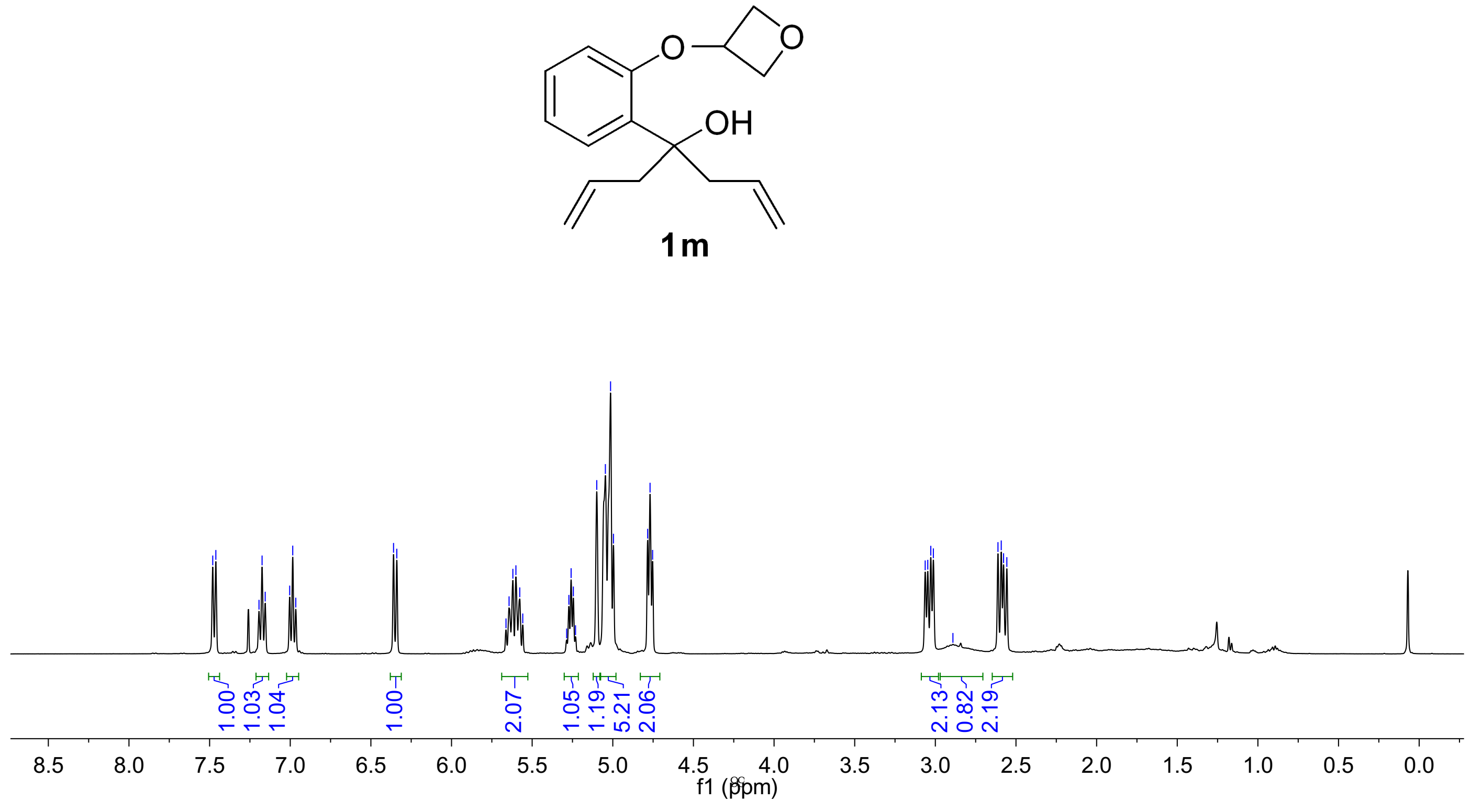
<smiles>C=CCC(O)(CC=C)c1ccccc1OC1COC1</smiles>

\section{$1 \mathrm{~m}$}

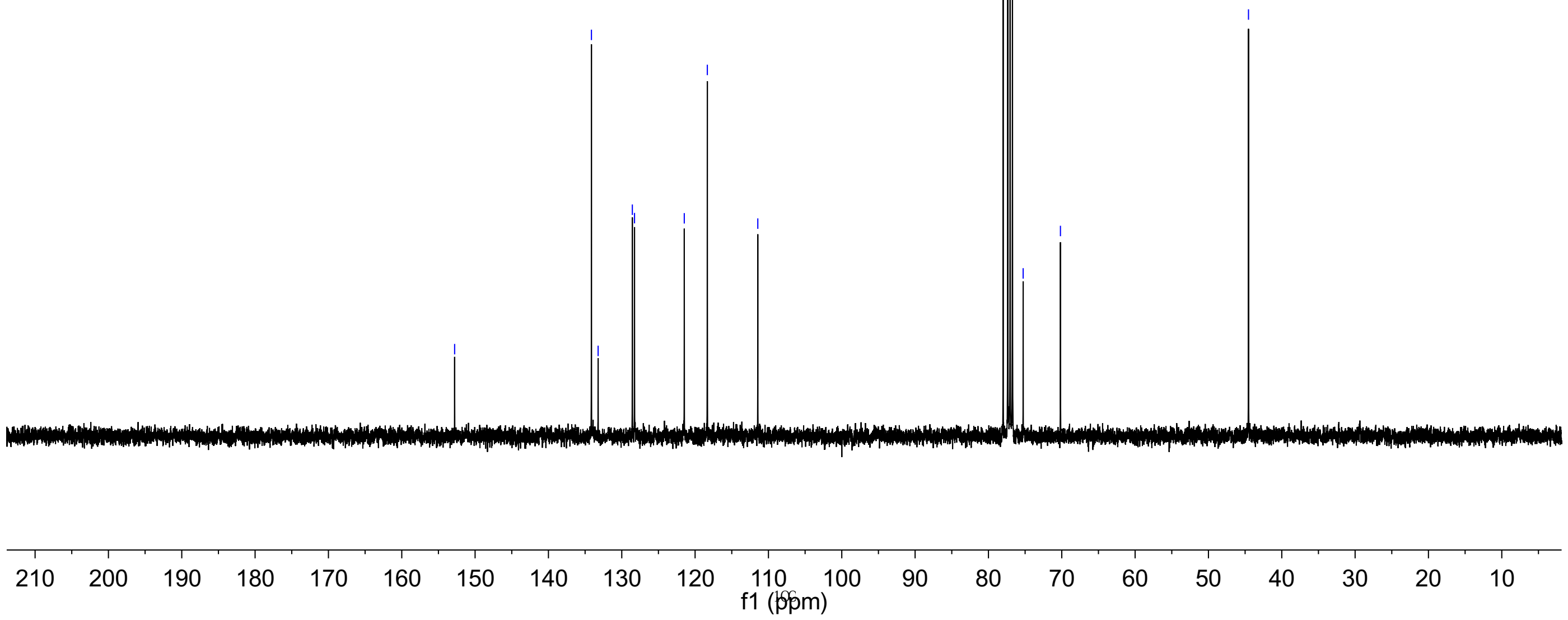




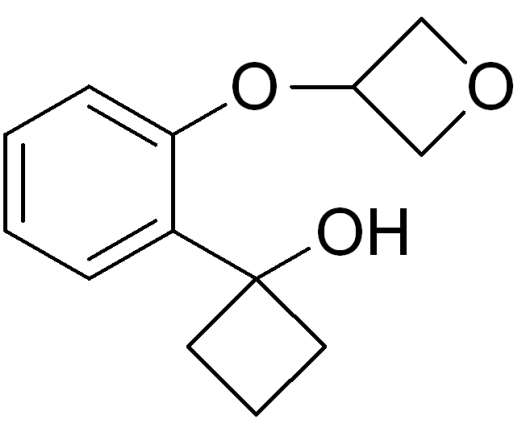

$1 \mathrm{n}$

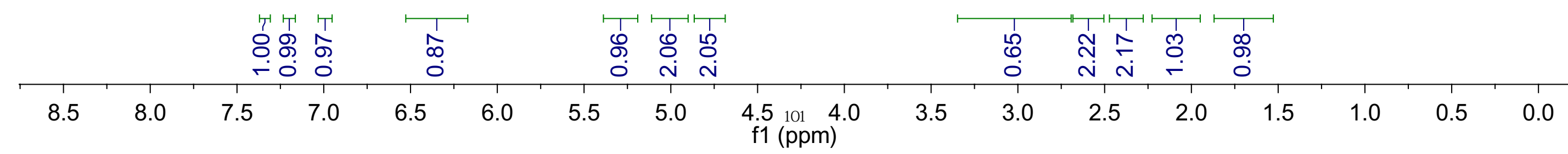




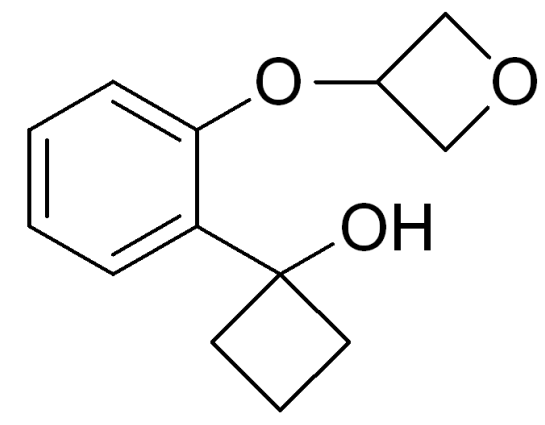

$1 \mathrm{n}$

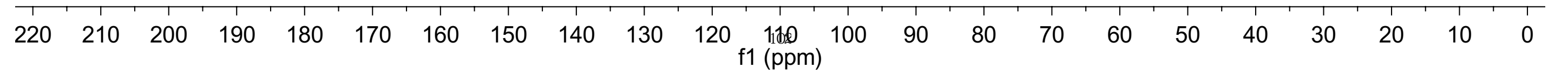




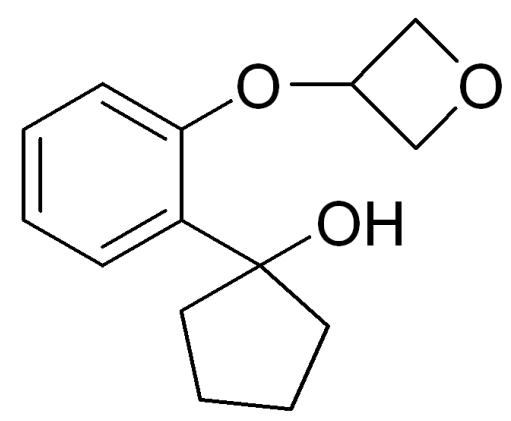

10

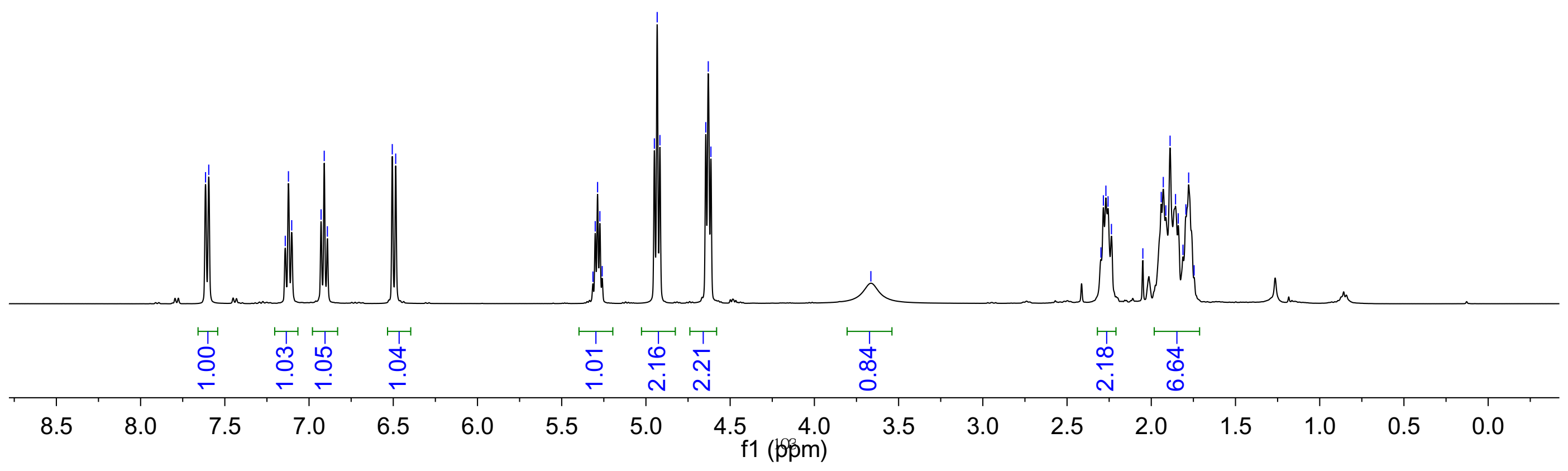




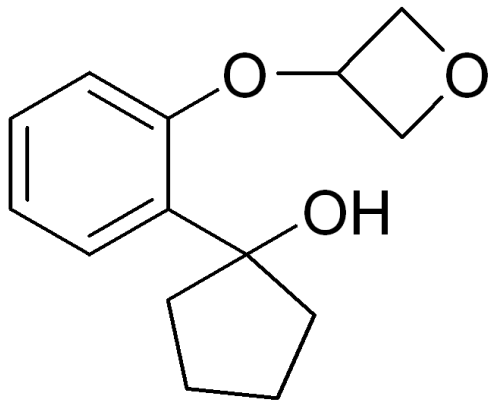

10

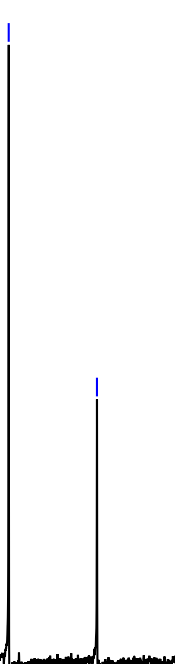




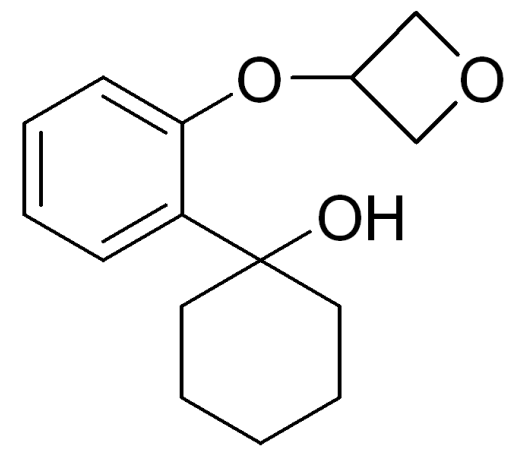

$1 p$

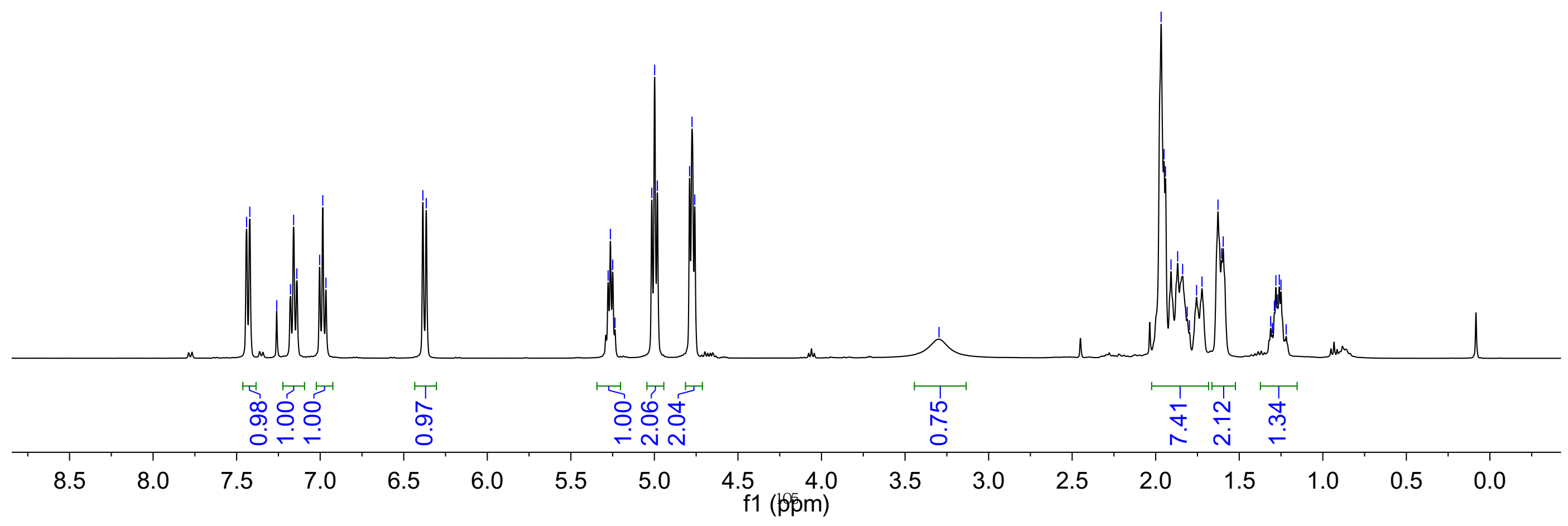




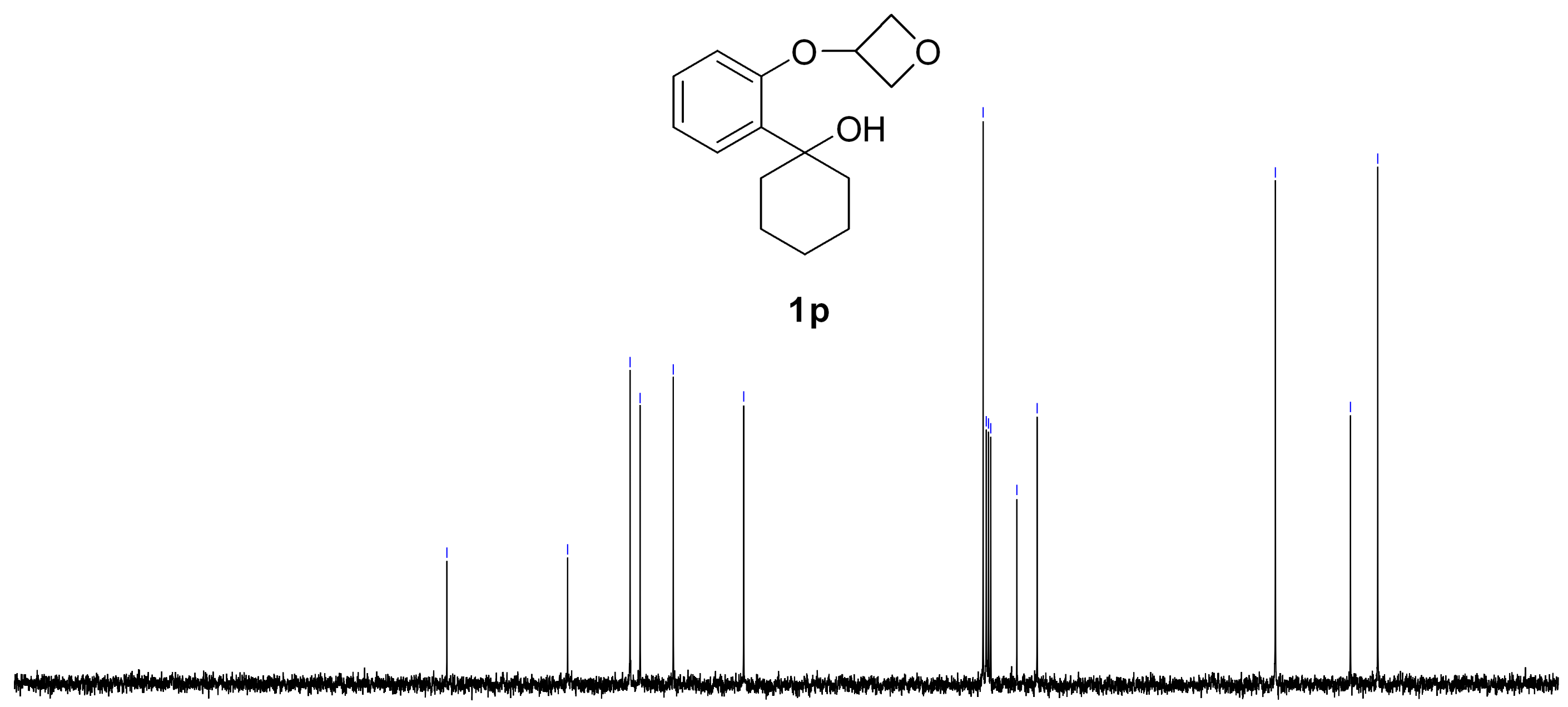

\begin{tabular}{|c|c|c|c|c|c|c|c|c|c|c|c|c|c|c|c|c|c|c|c|c|}
\hline 210 & 200 & 190 & 180 & 170 & 160 & 150 & 140 & 130 & 120 & $\begin{array}{l}110 \quad 100 \\
\mathrm{f} 1(\mathrm{p} \rho \mathrm{m})\end{array}$ & 90 & 80 & 70 & 60 & 50 & 40 & 30 & 20 & 10 & 0 \\
\hline
\end{tabular}




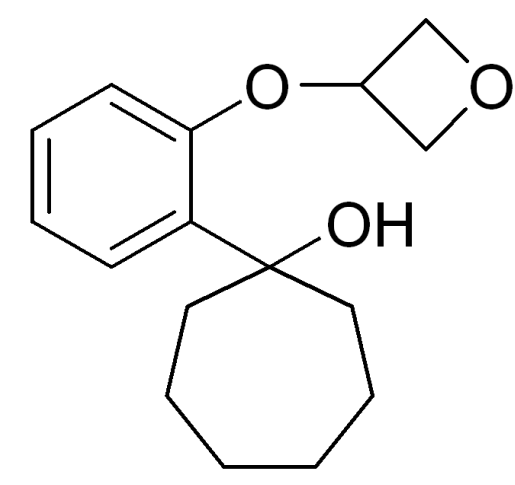

19

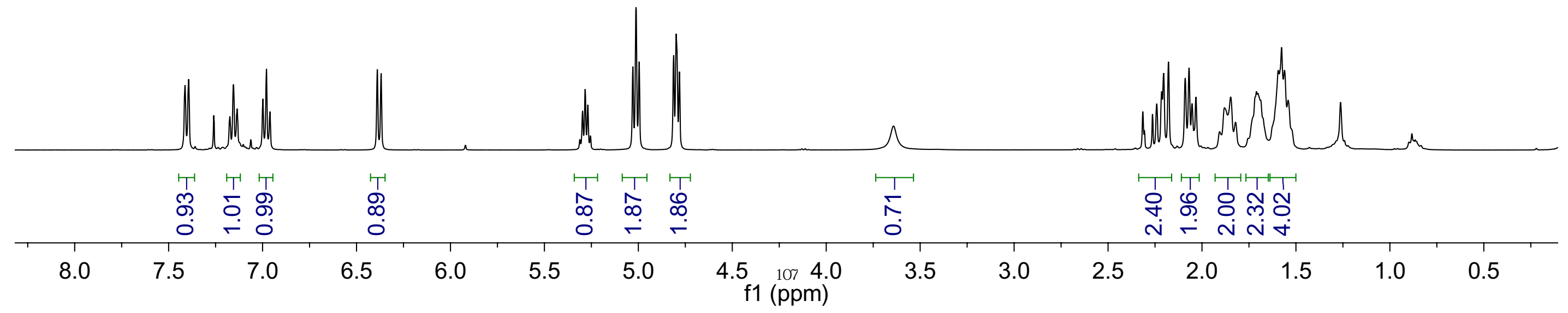




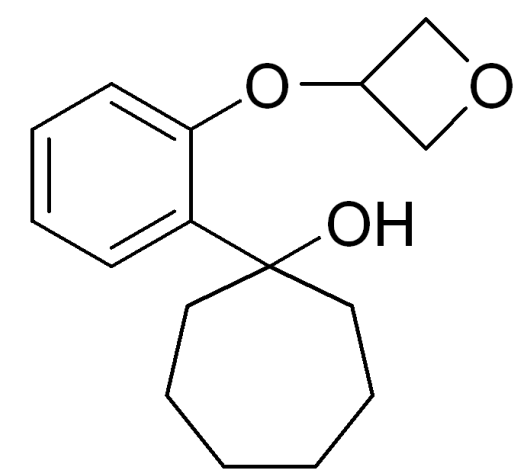

$1 q$

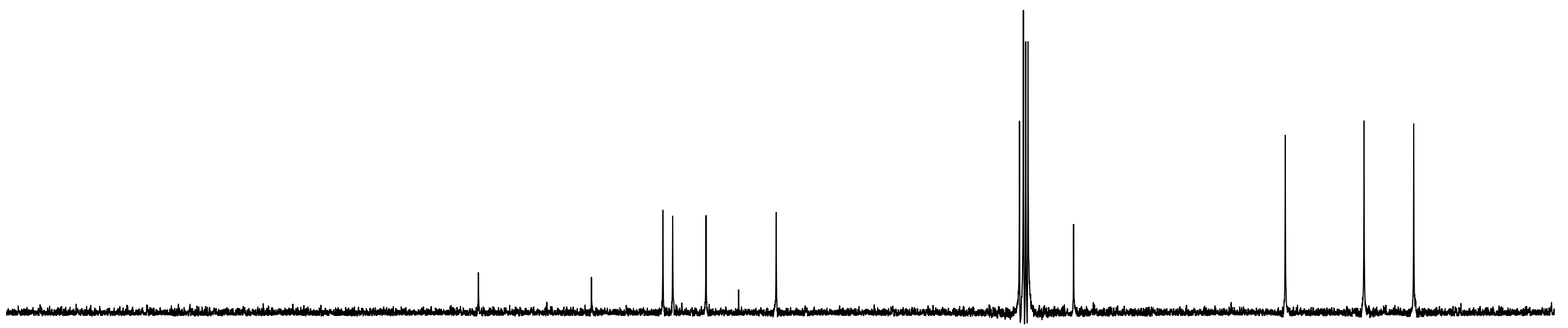



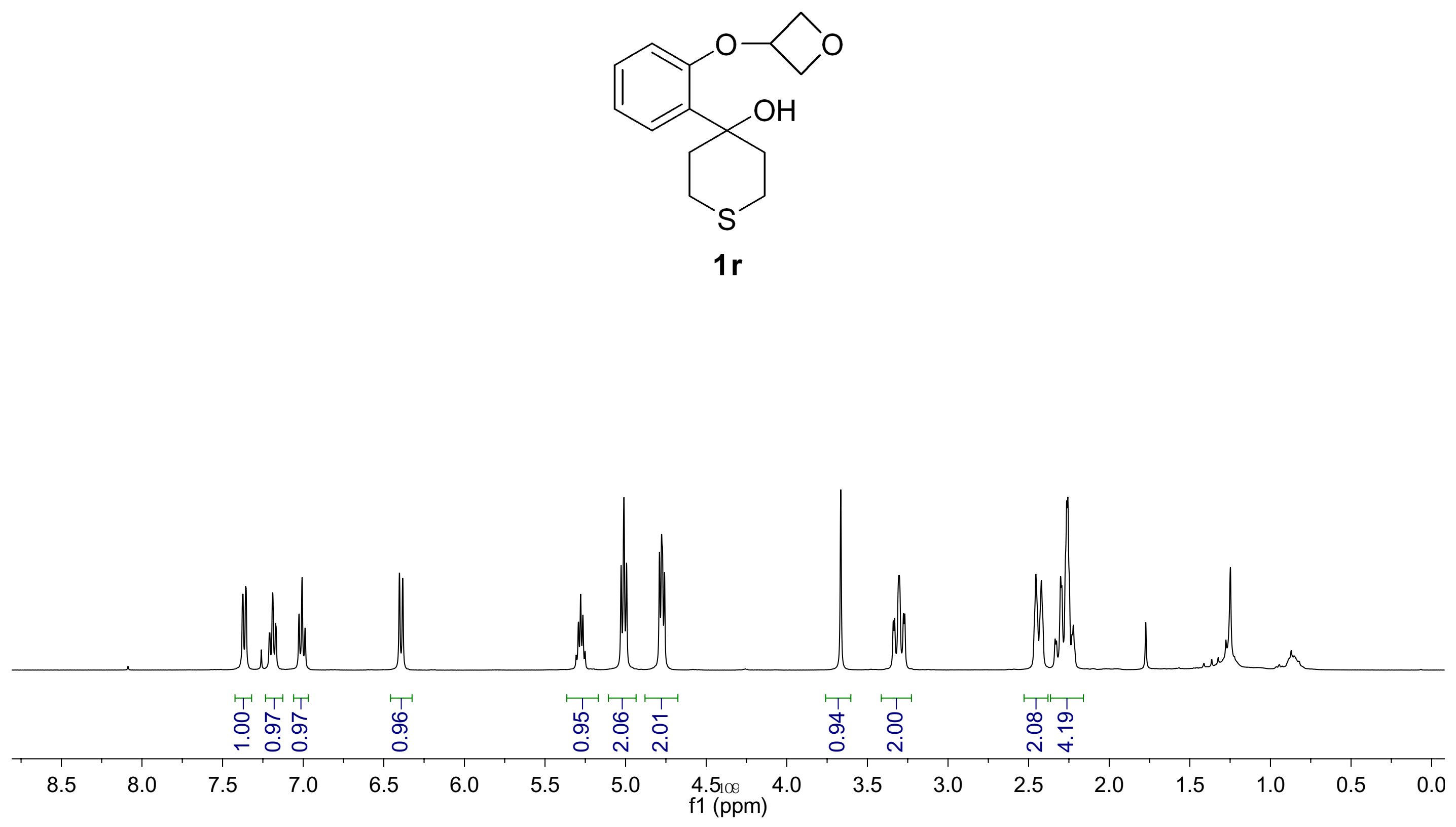

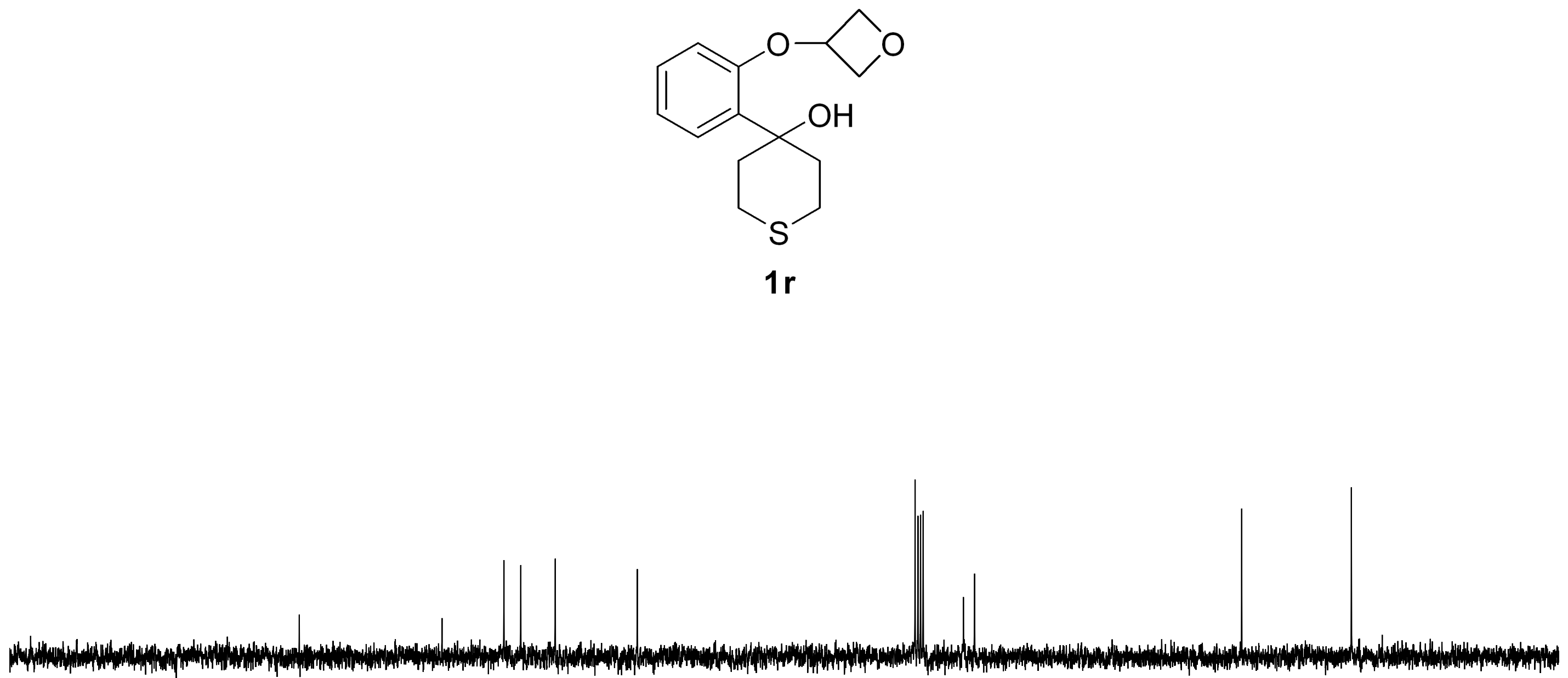

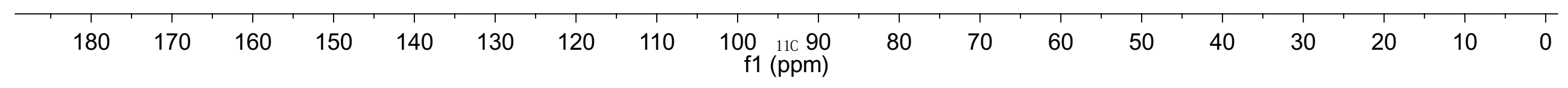



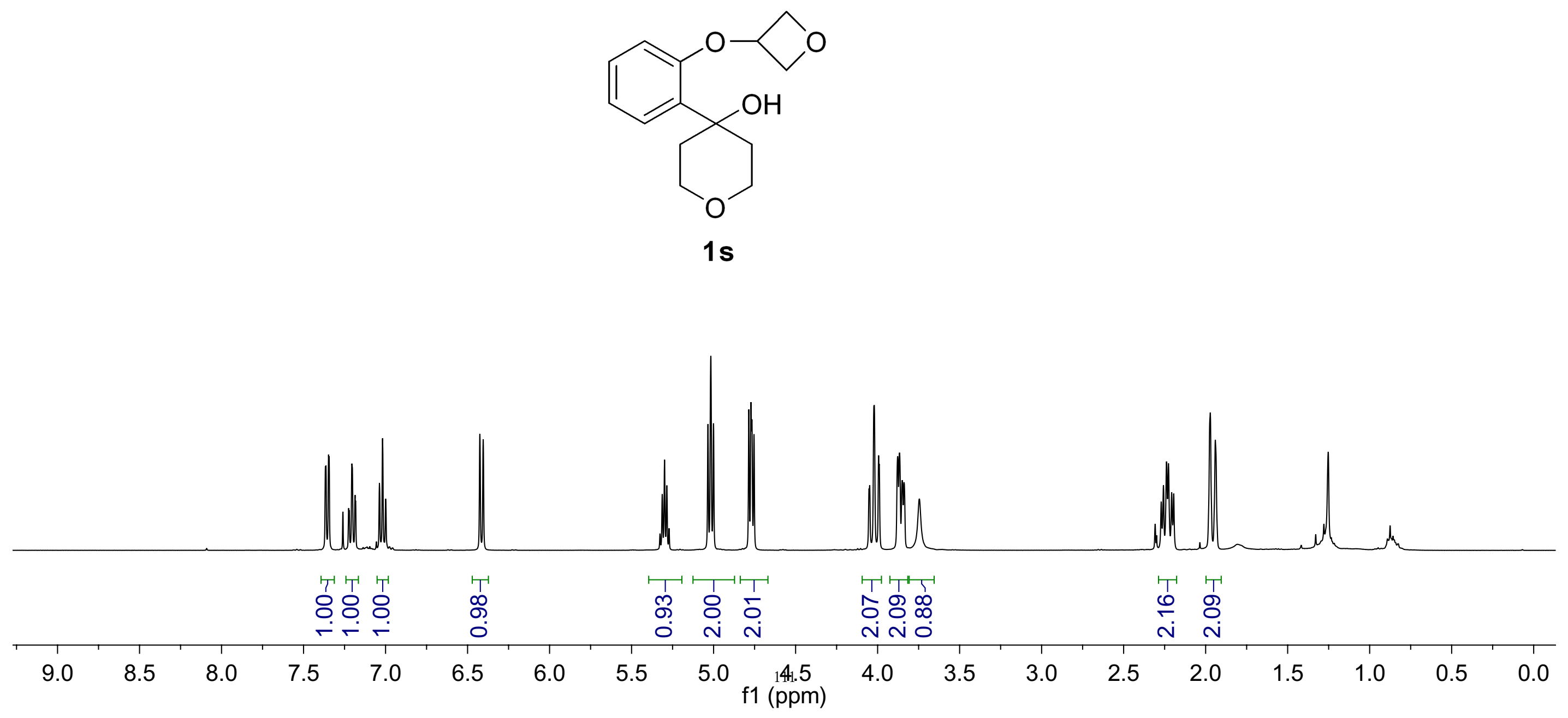


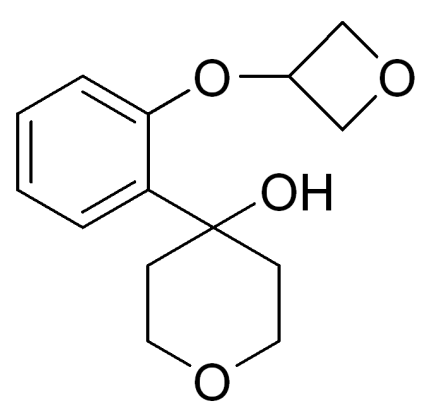

$1 \mathrm{~s}$ 


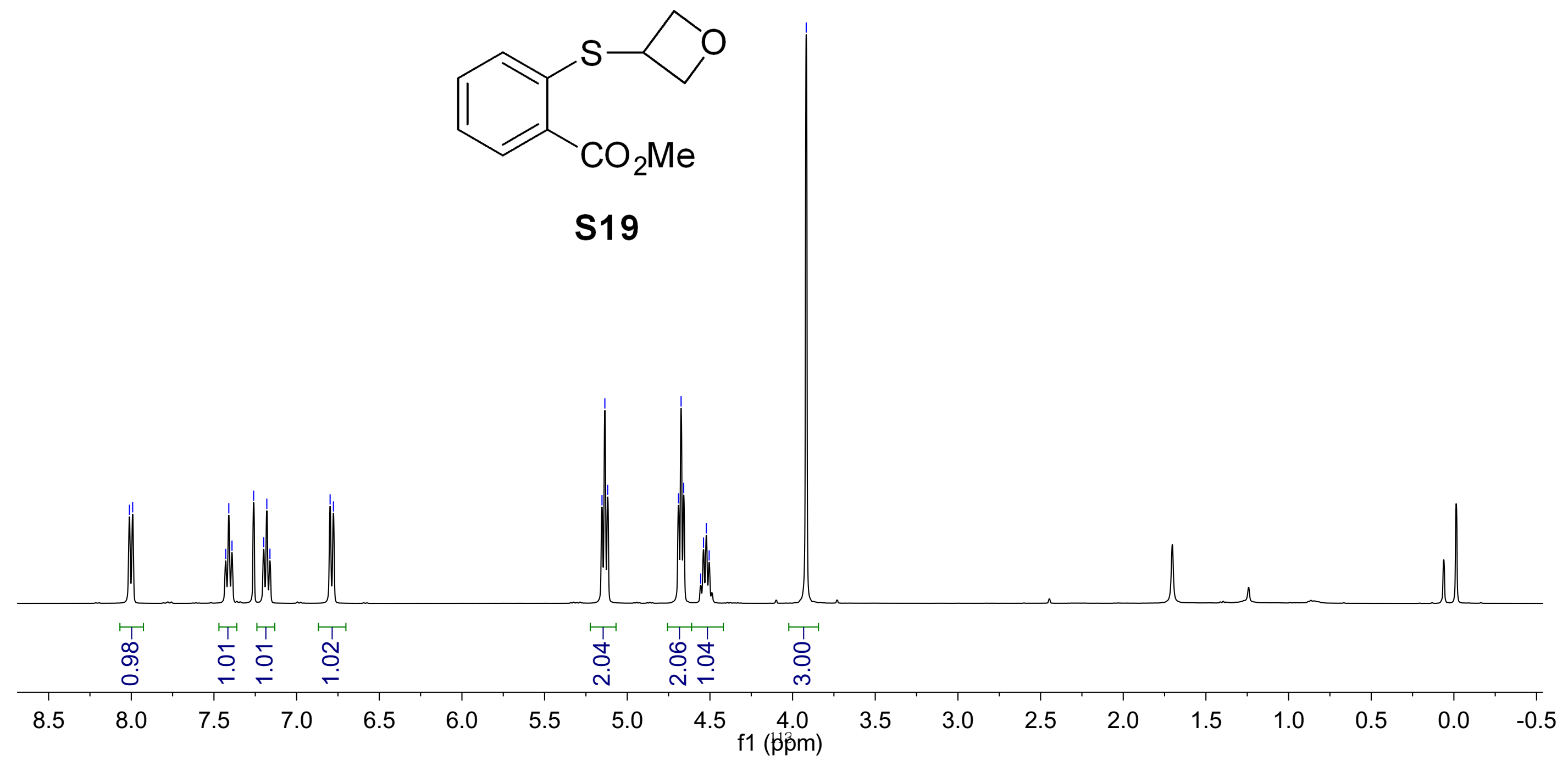




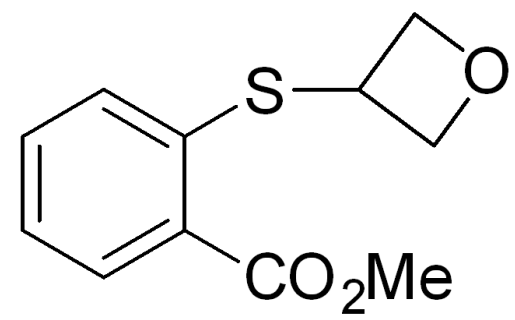

S19

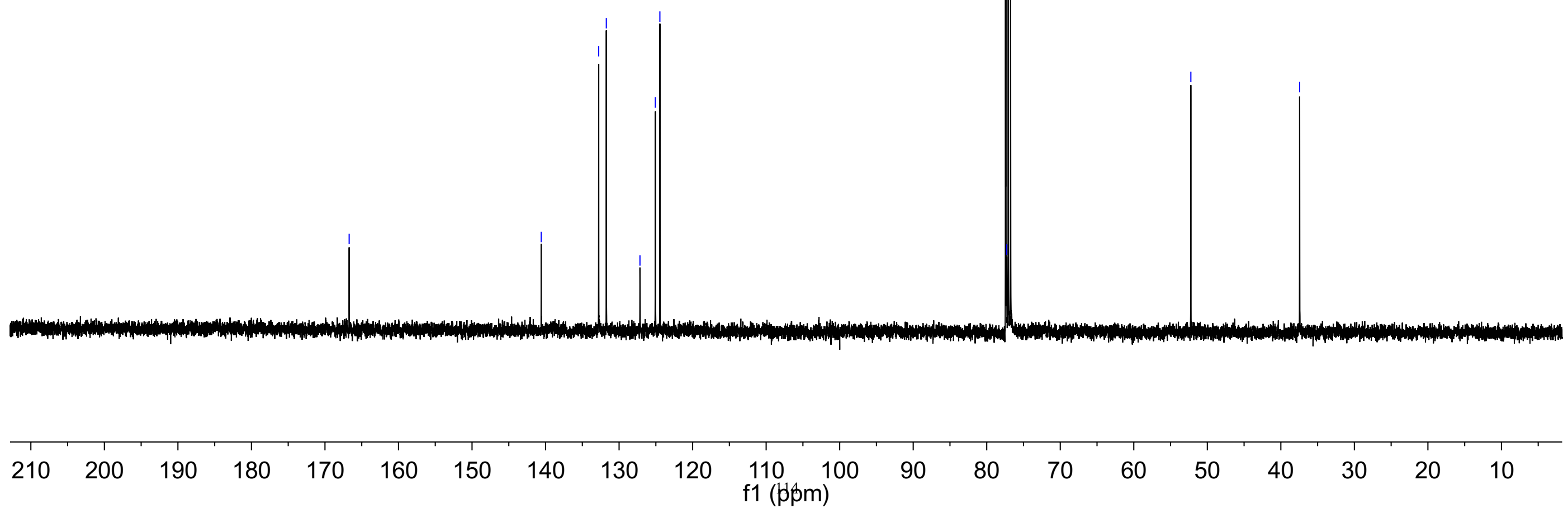




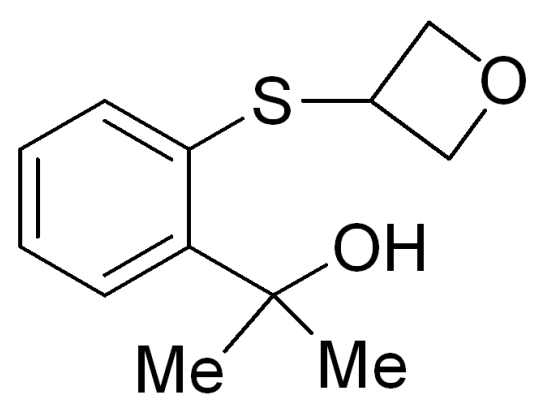

\section{$1 \mathrm{~b}-\mathrm{S}$}

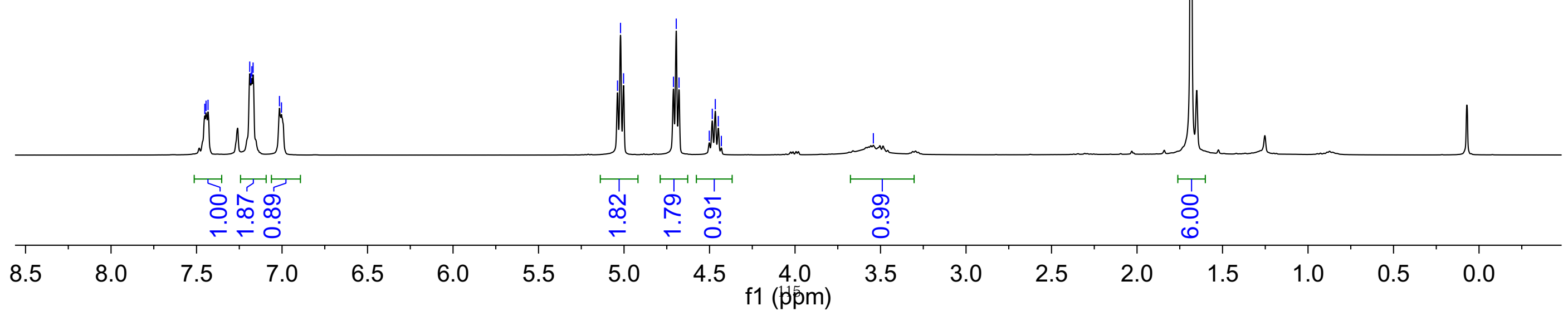




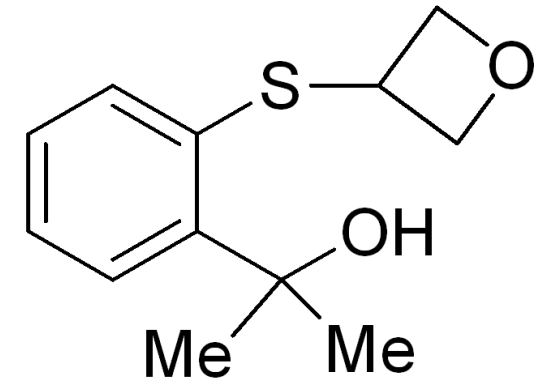

\section{$1 \mathrm{~b}-\mathrm{S}$}

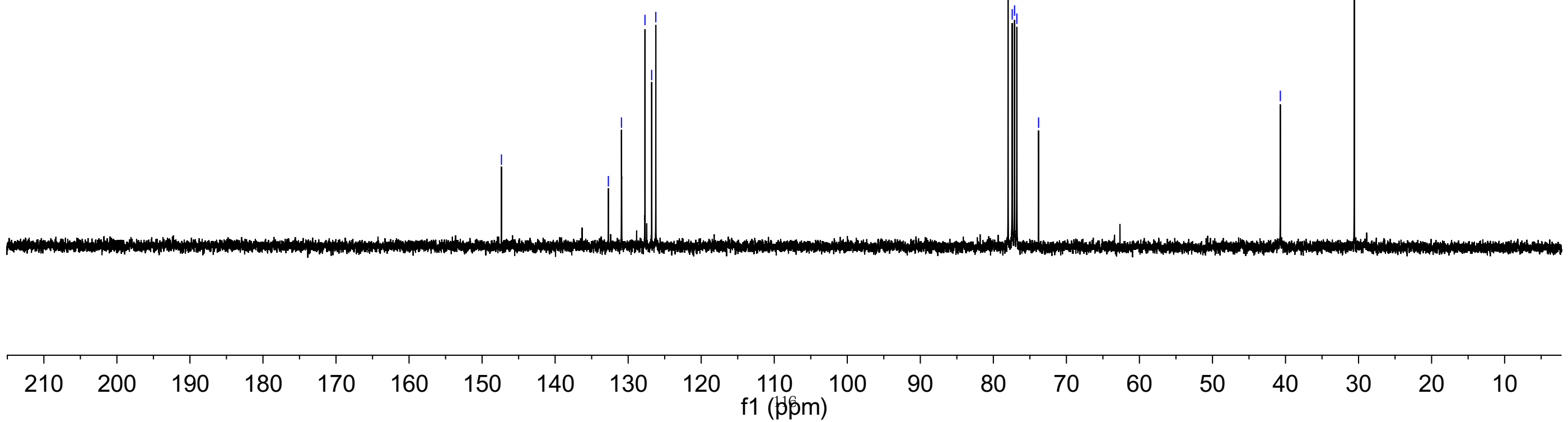



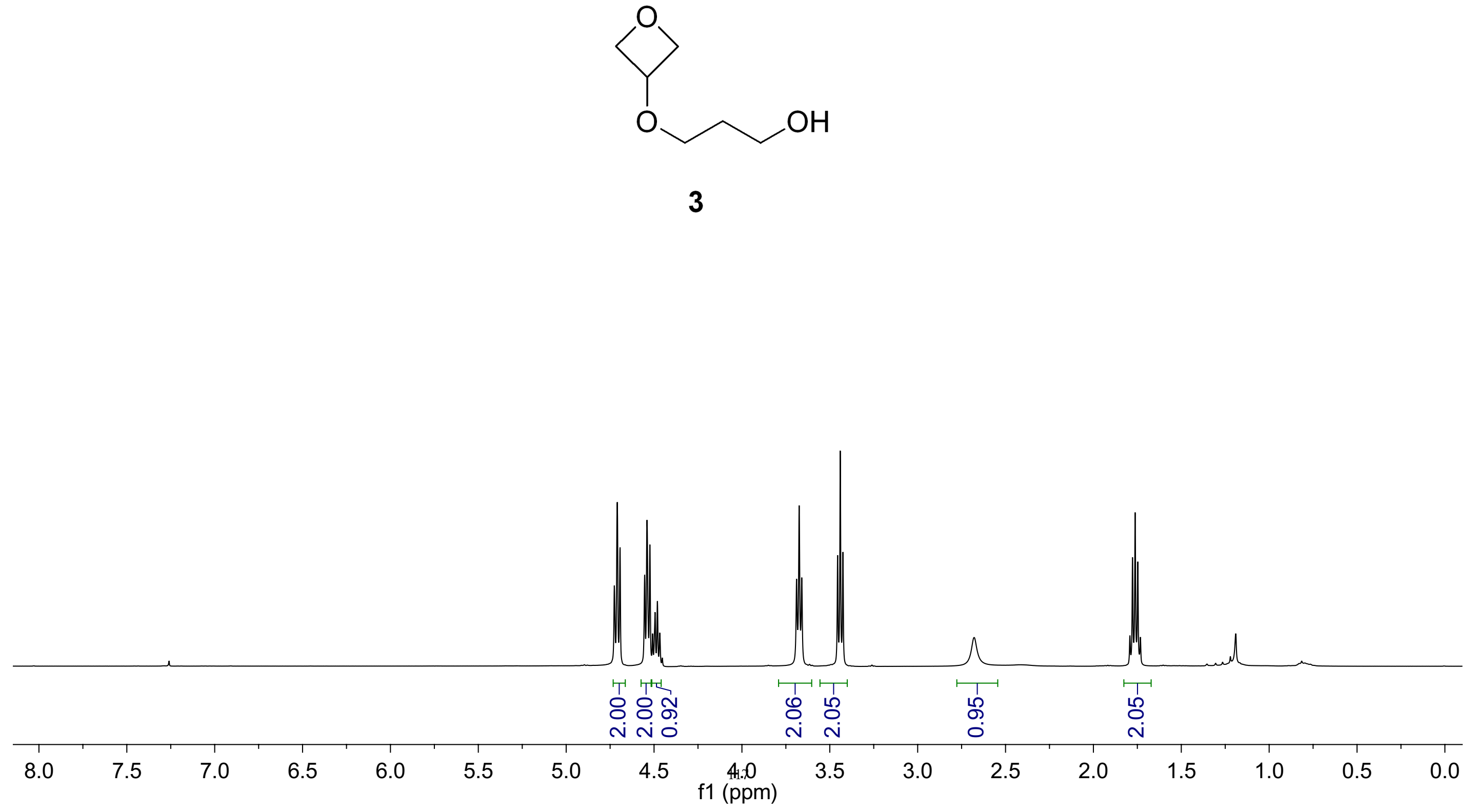


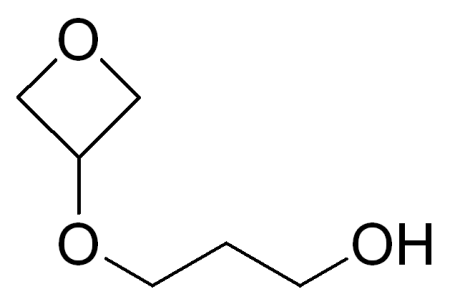

3

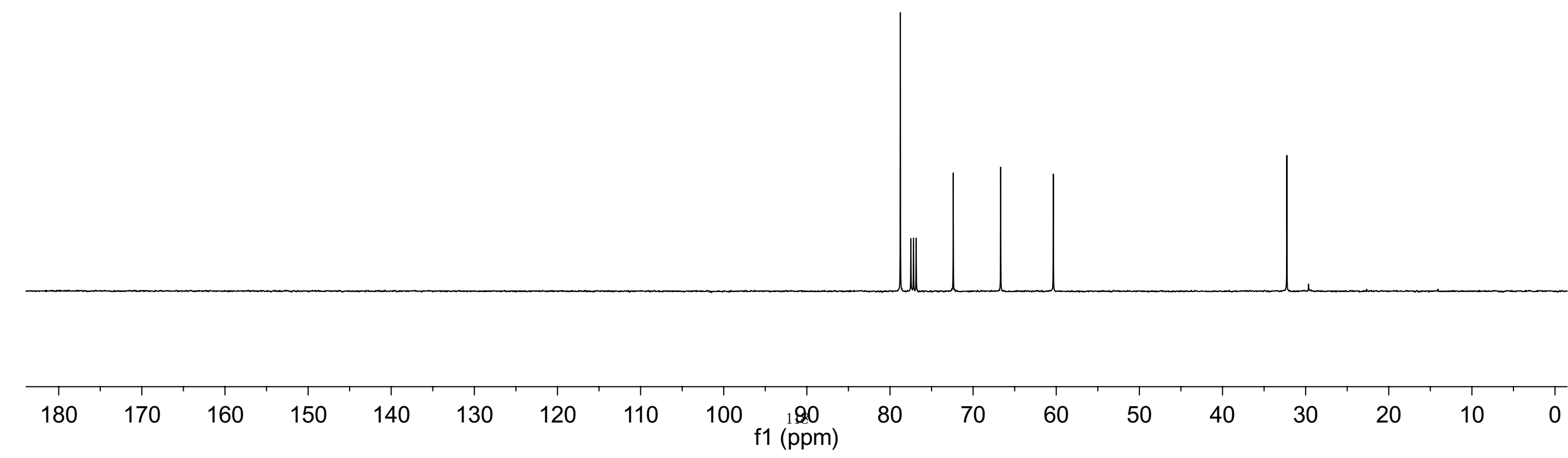



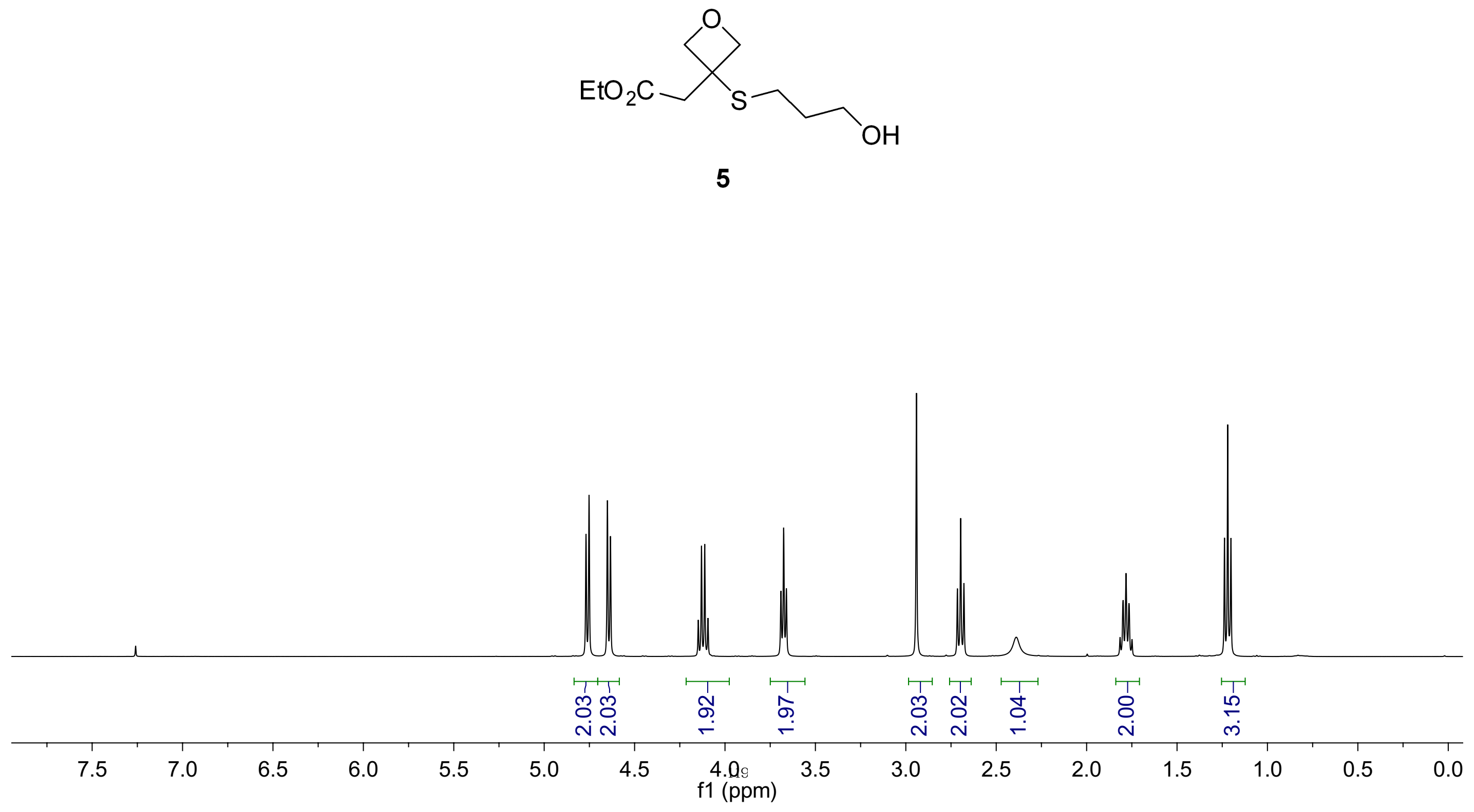


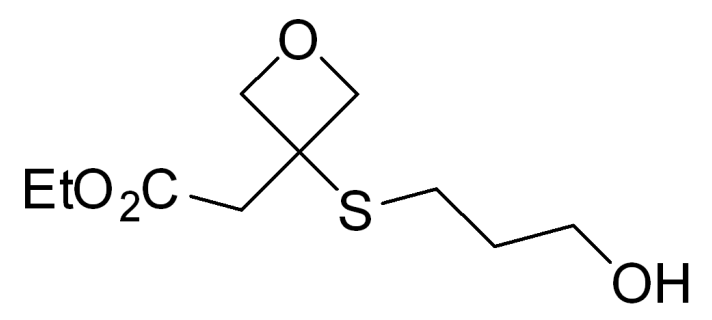

5

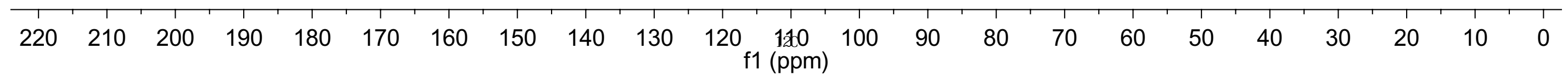




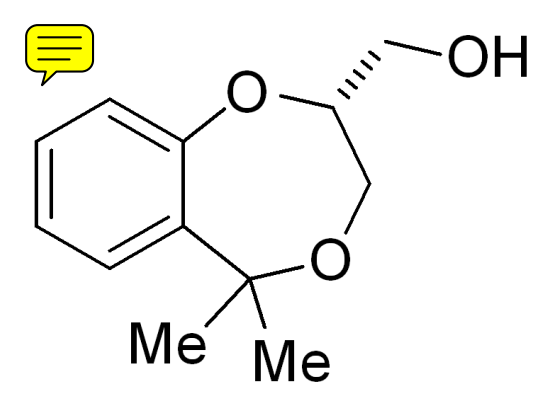

$2 b$

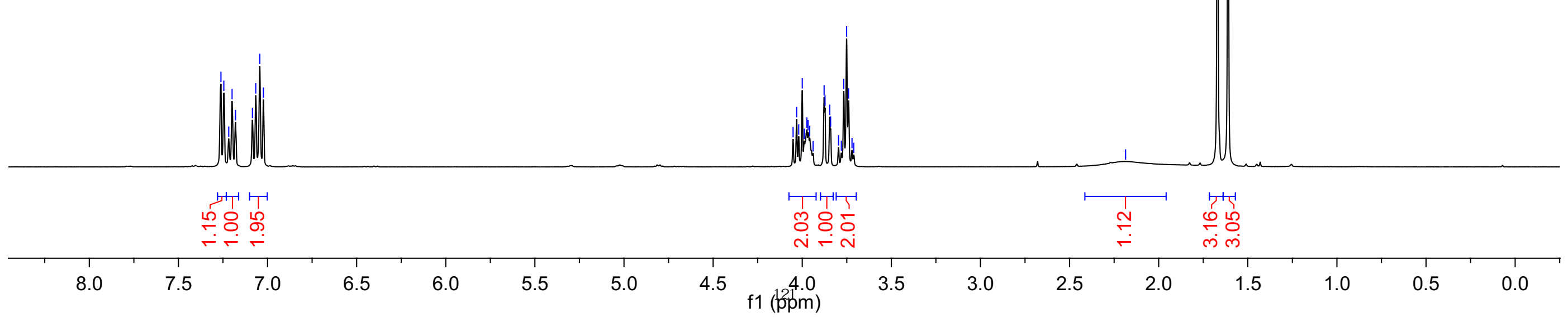




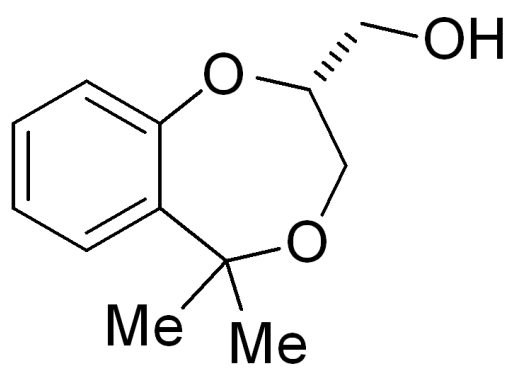

2b

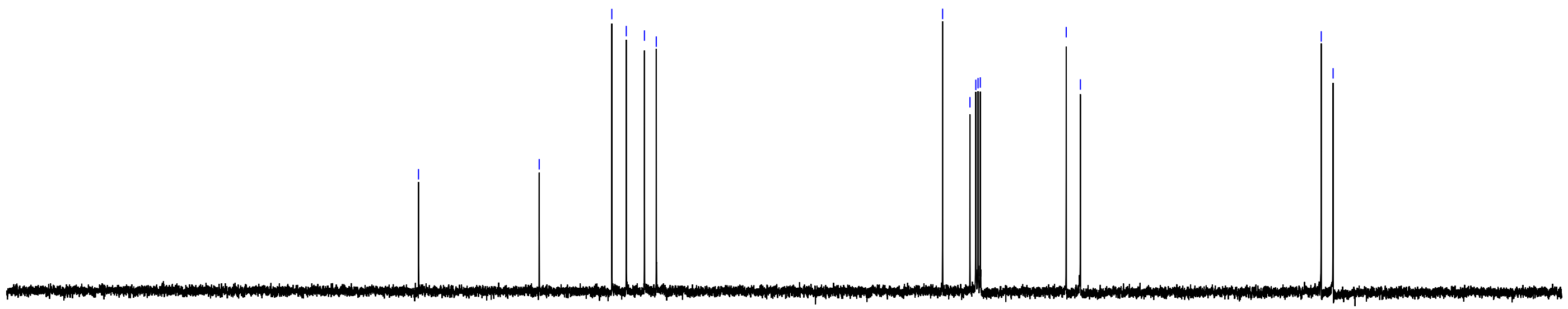




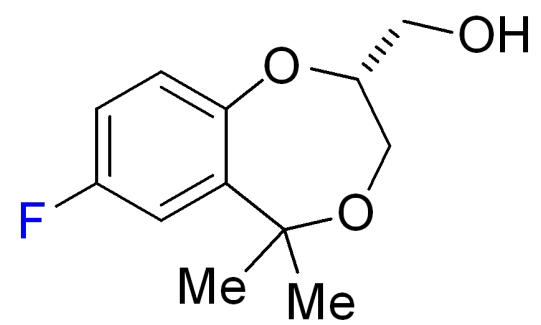

2c

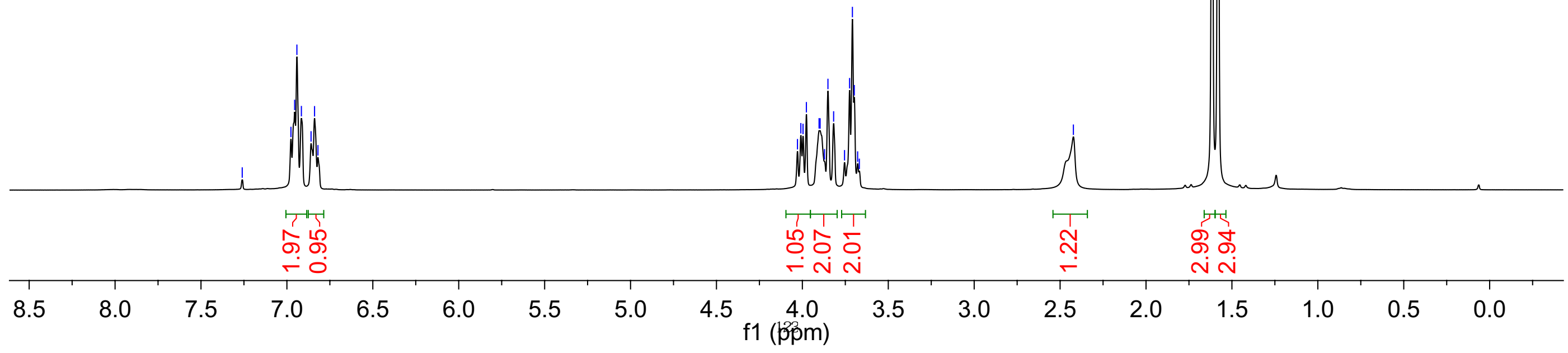



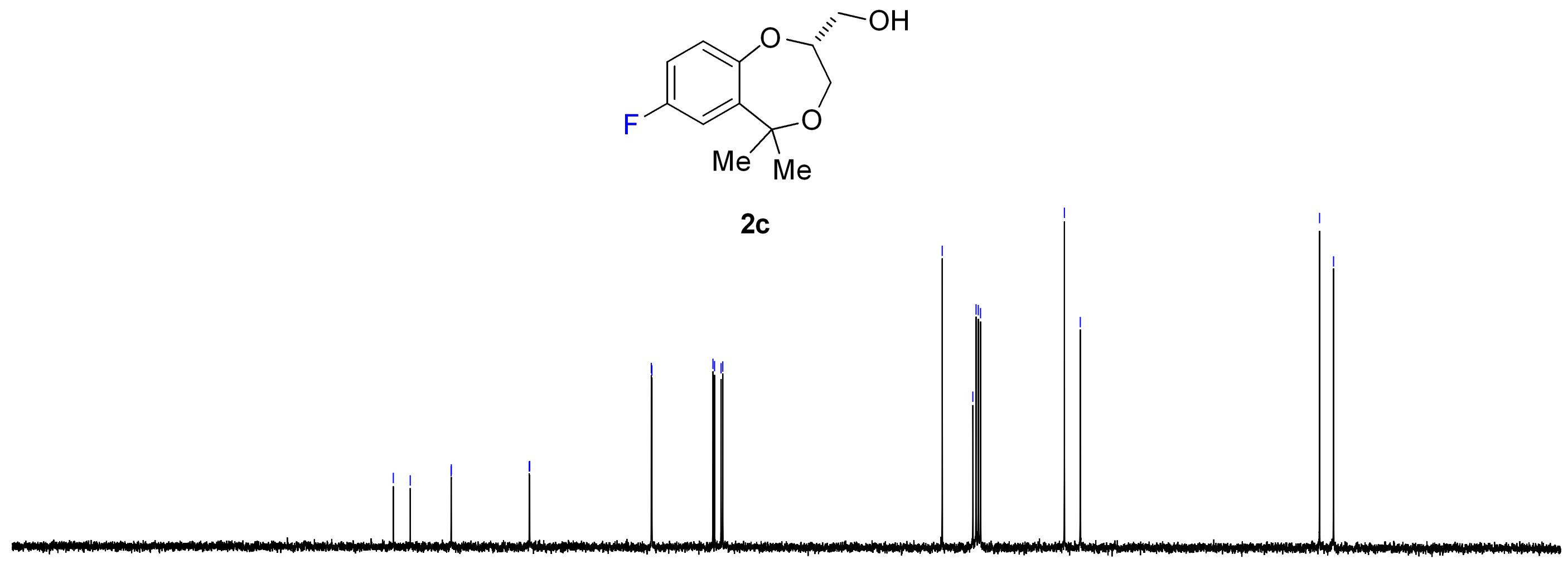


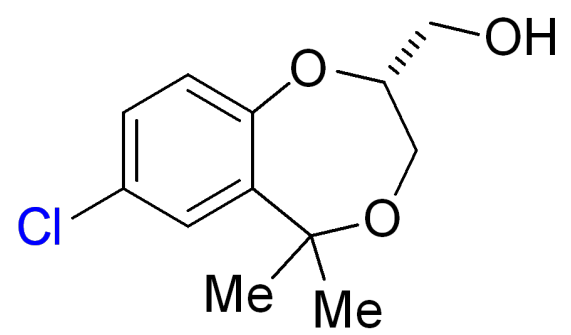

2d

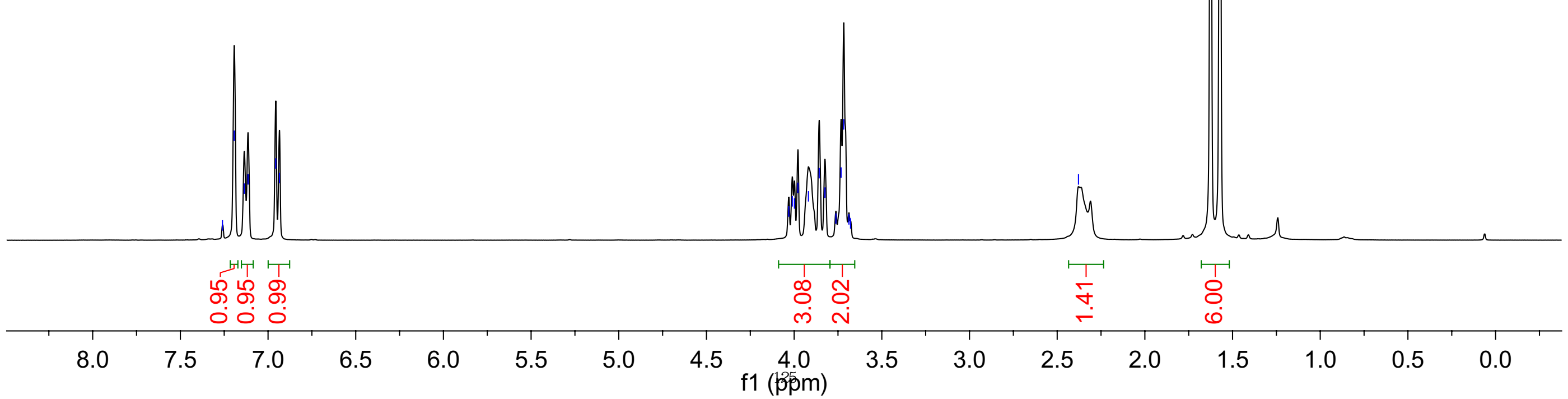




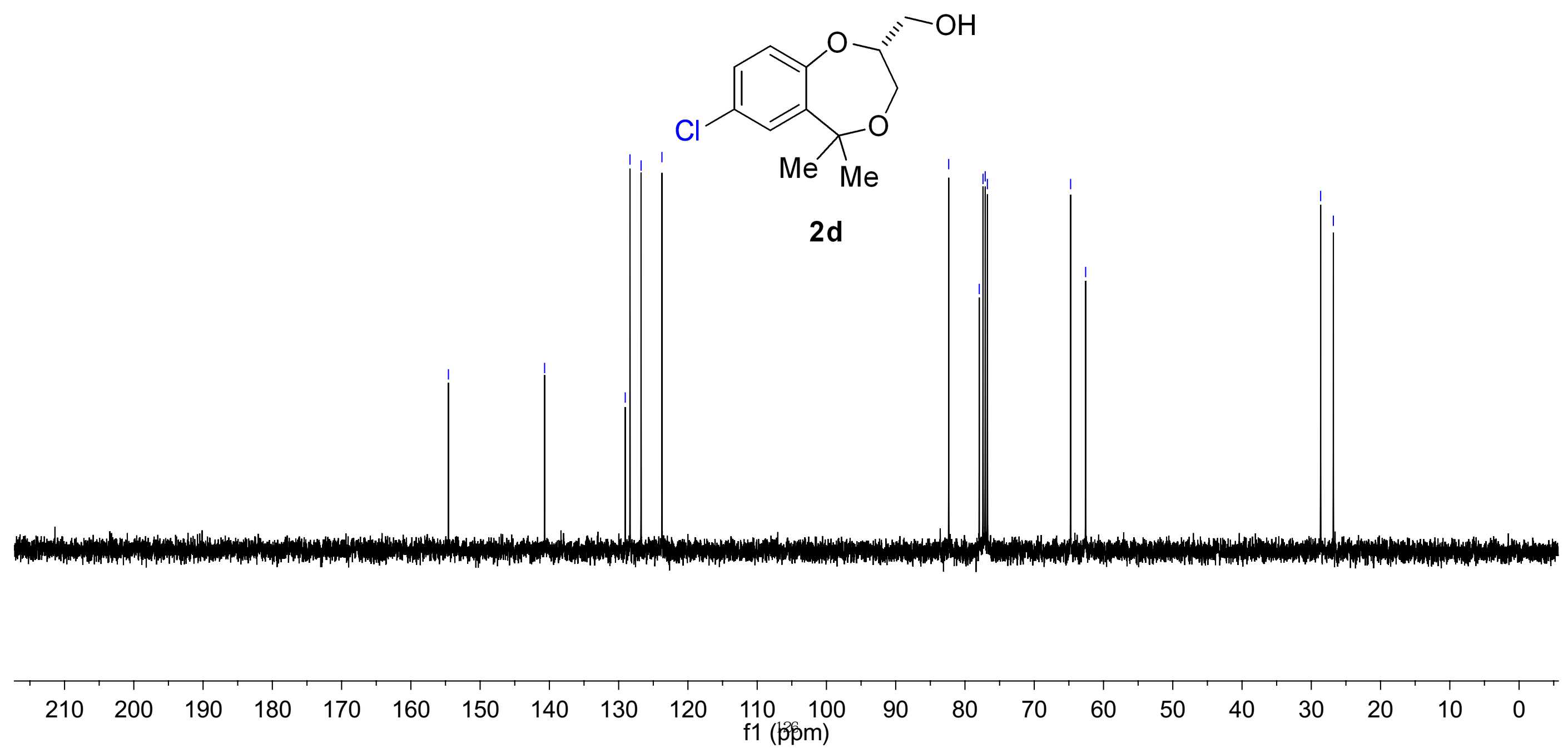




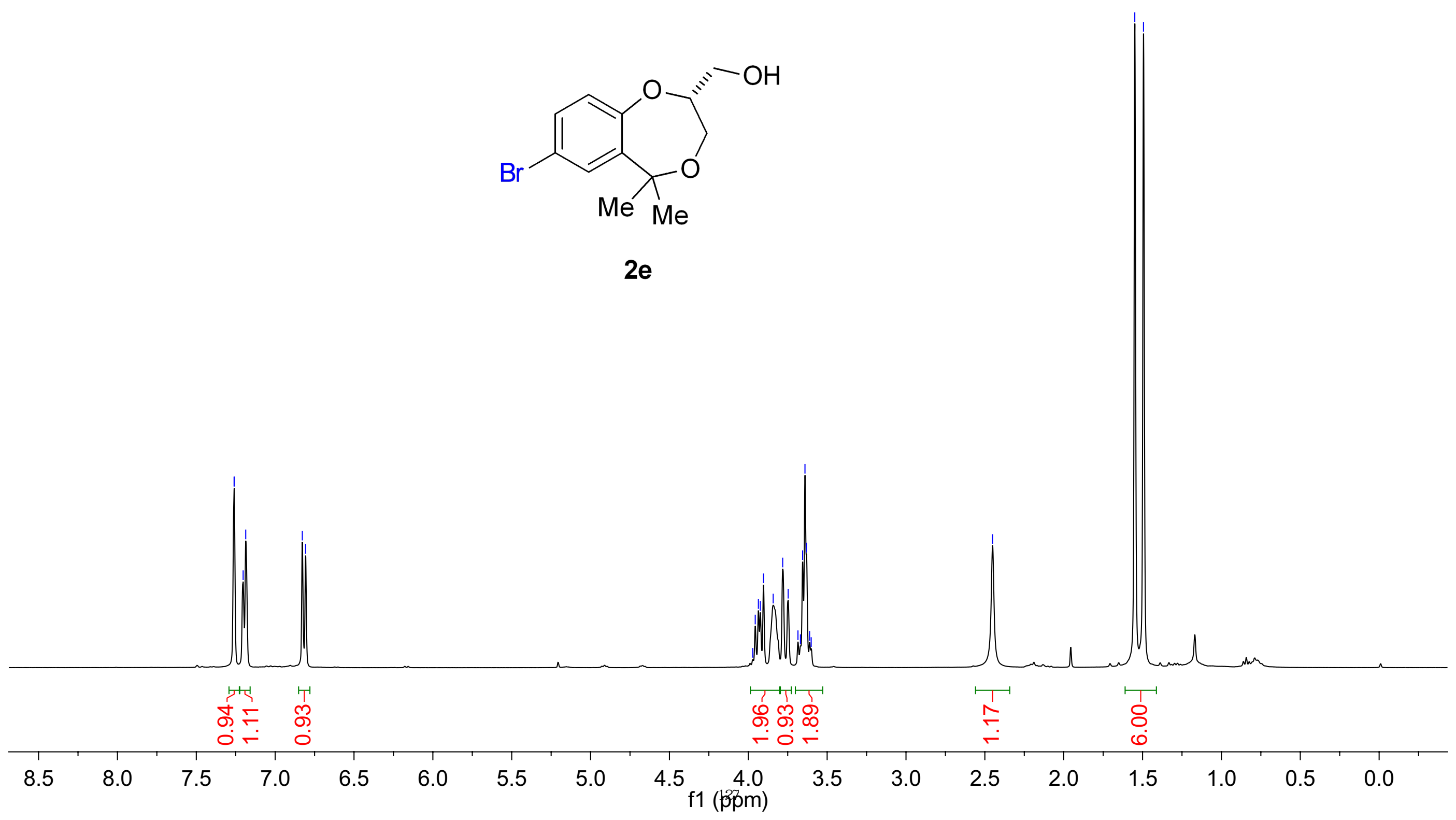


<smiles>CC1(C)OC[C@H](CO)Oc2ccc(Br)cc21</smiles>

$2 e$

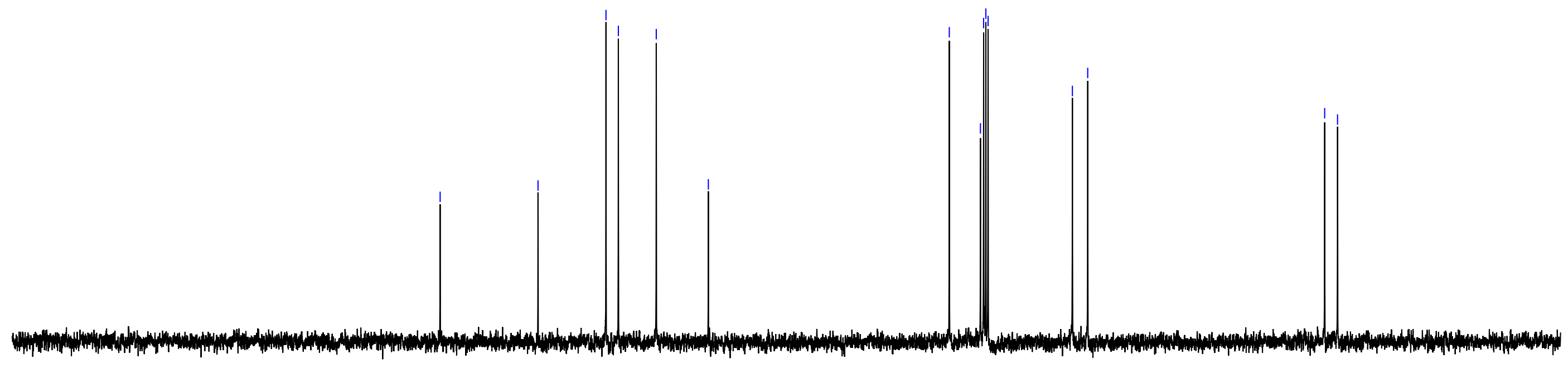

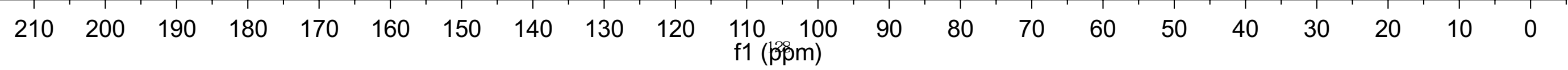




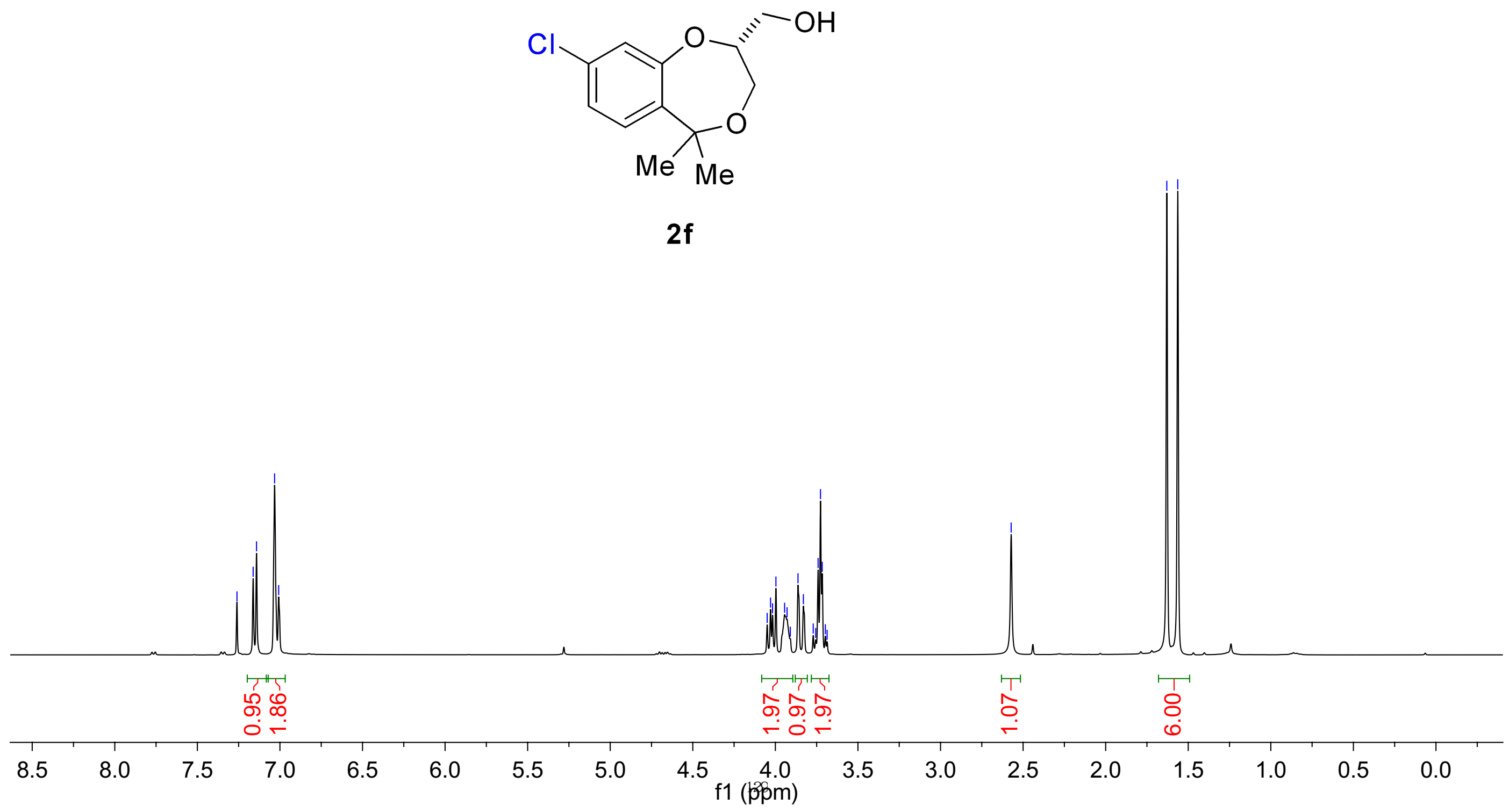


<smiles>CC1(C)OC[C@@H](CO)Oc2cc(Cl)ccc21</smiles>

$2 f$

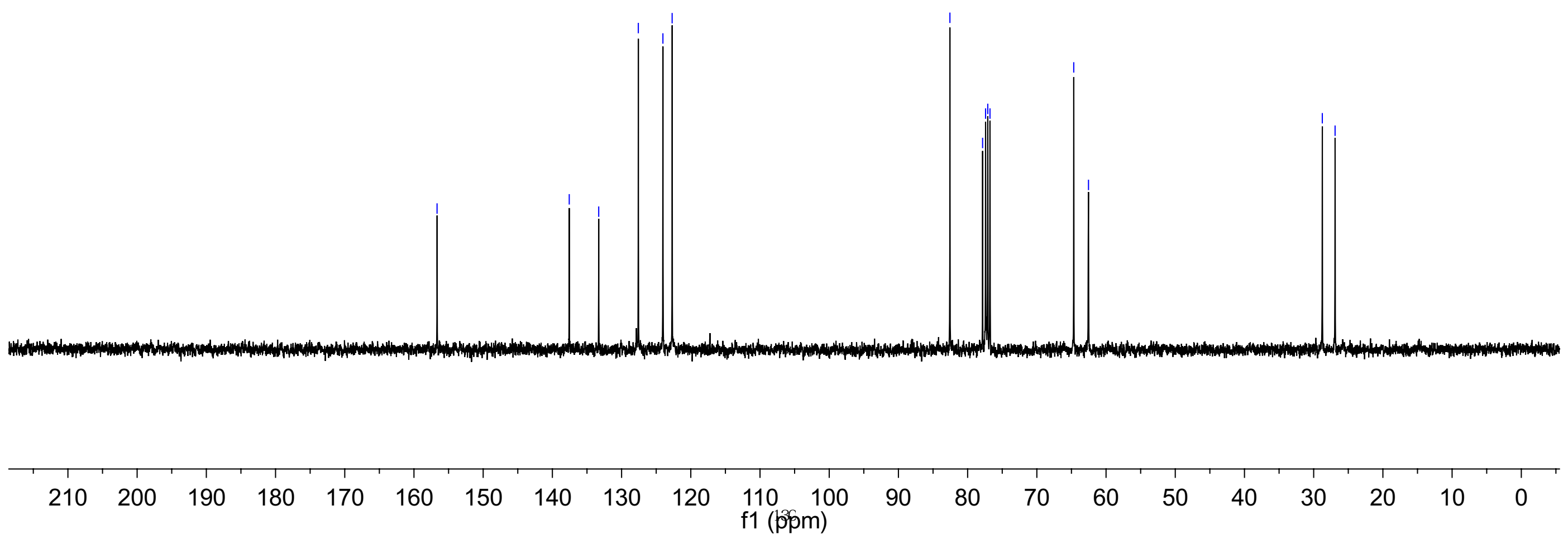




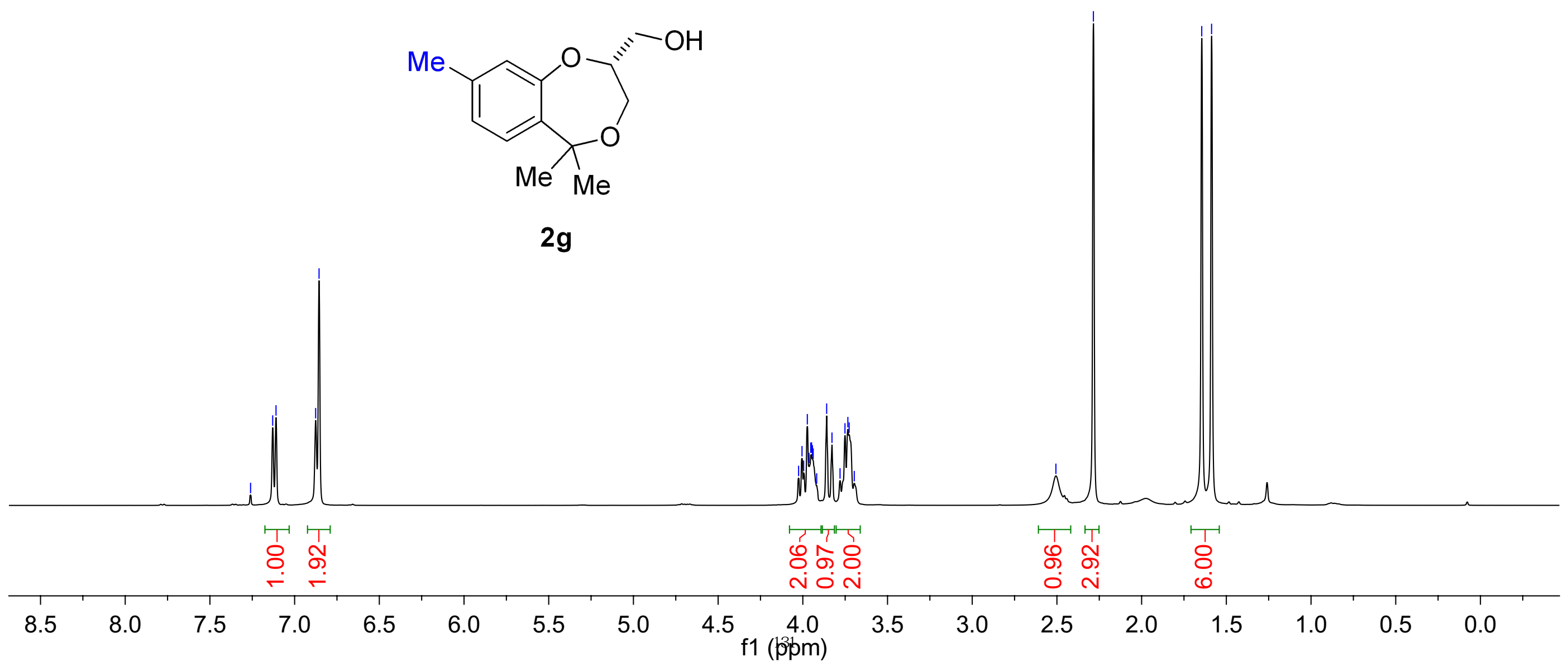



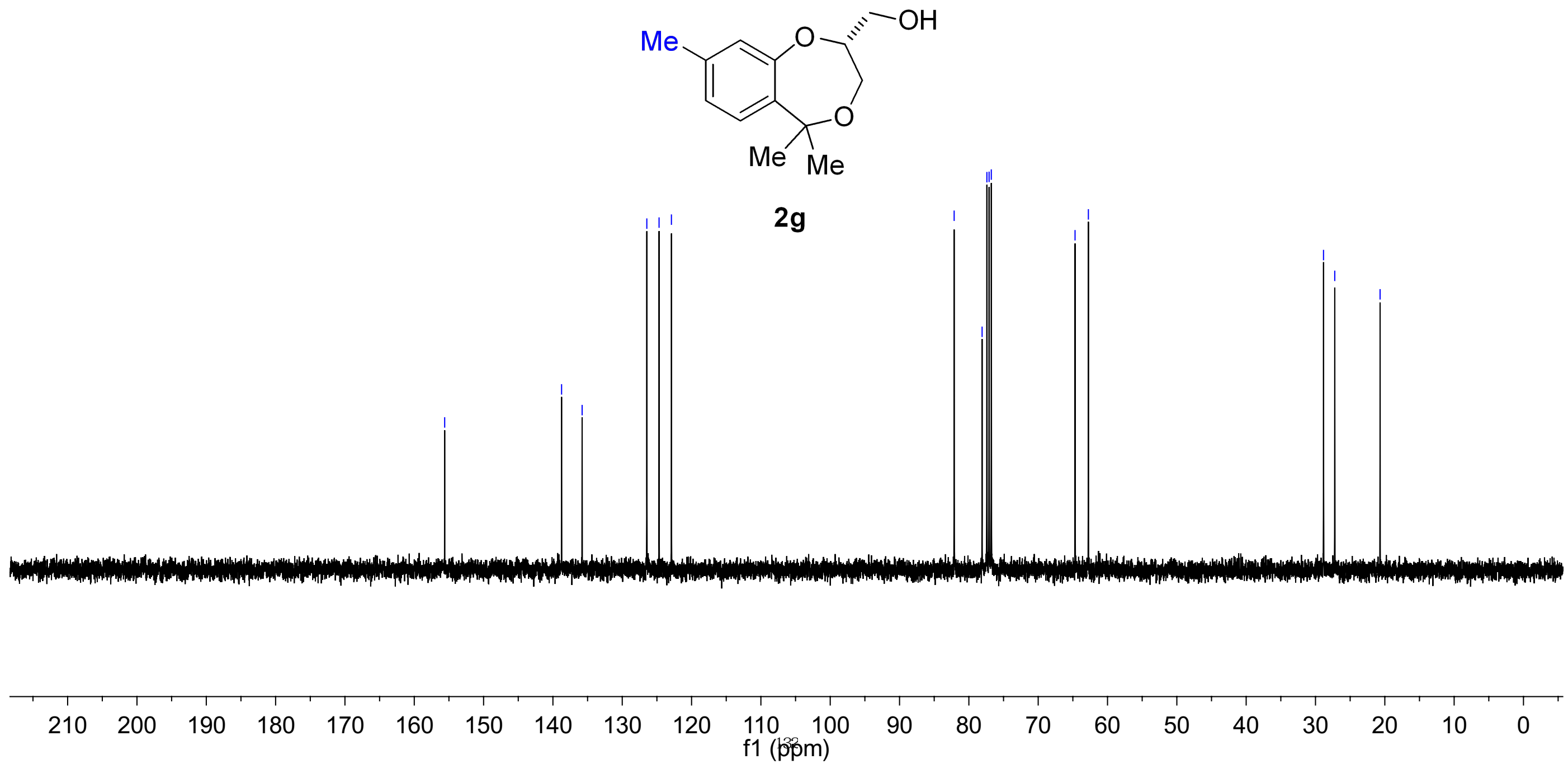


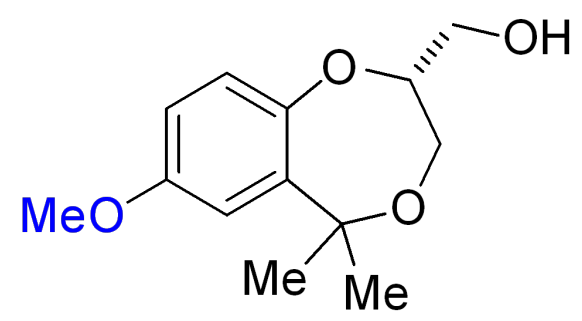

$2 \mathrm{~h}$

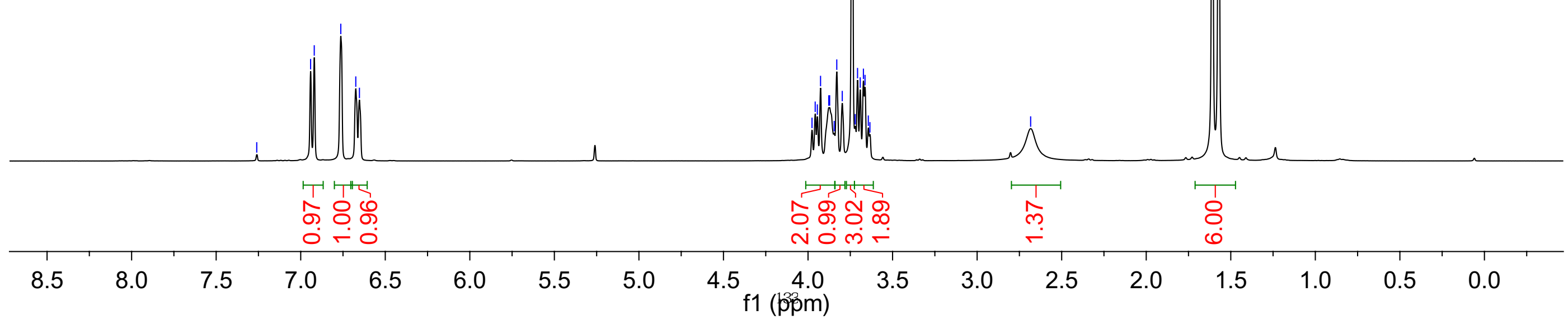




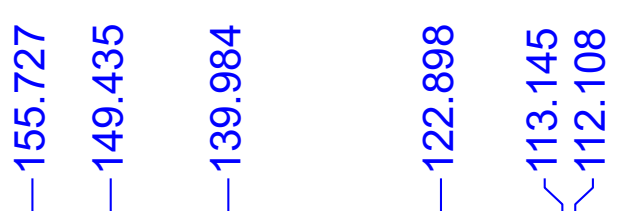

ชิํํํำ

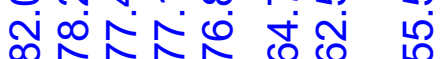

ఫั

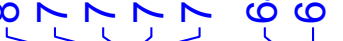

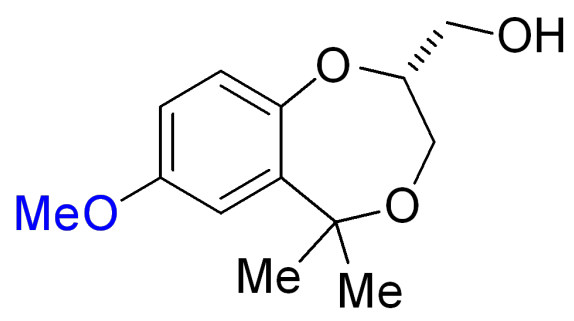

$2 \mathrm{~h}$ 


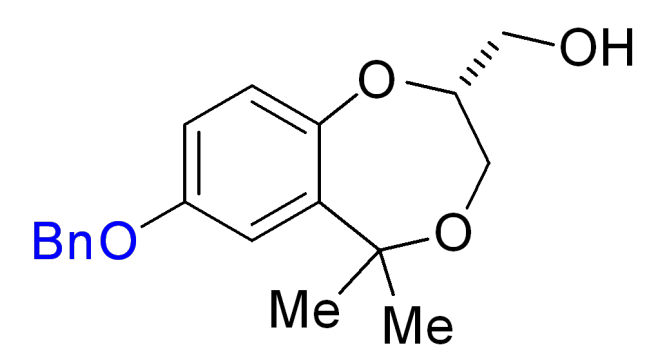

$2 \mathbf{i}$

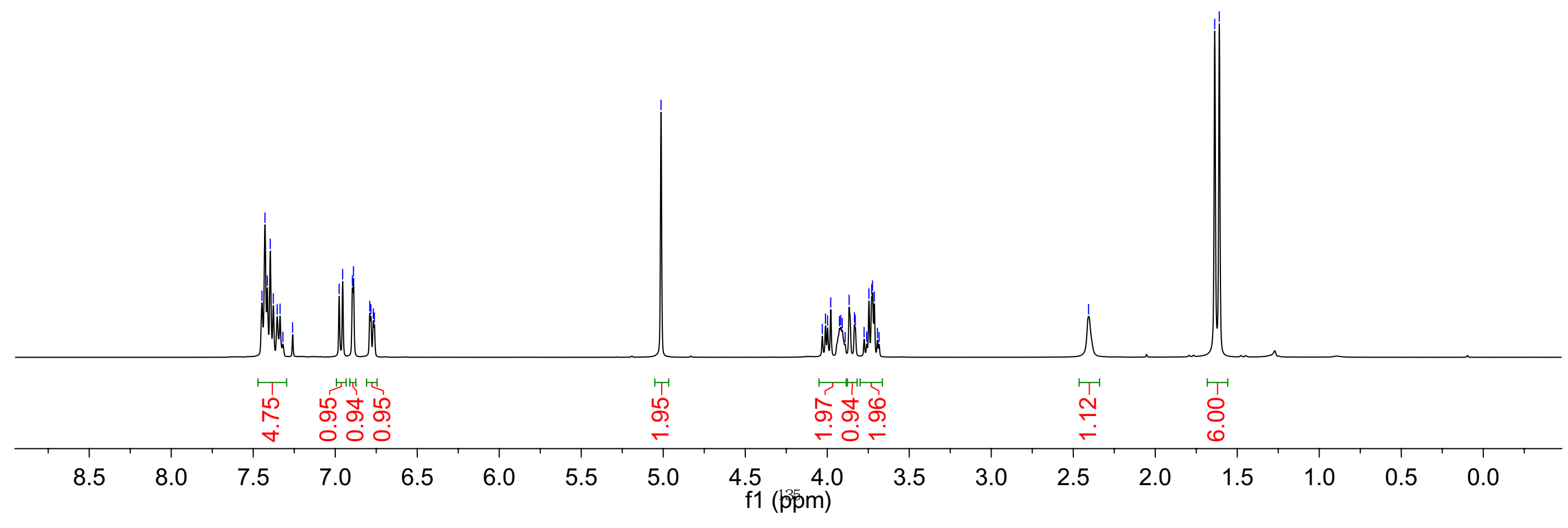


<smiles>CC1(C)OC[C@H](CO)Oc2ccc(OCc3ccccc3)cc21</smiles>

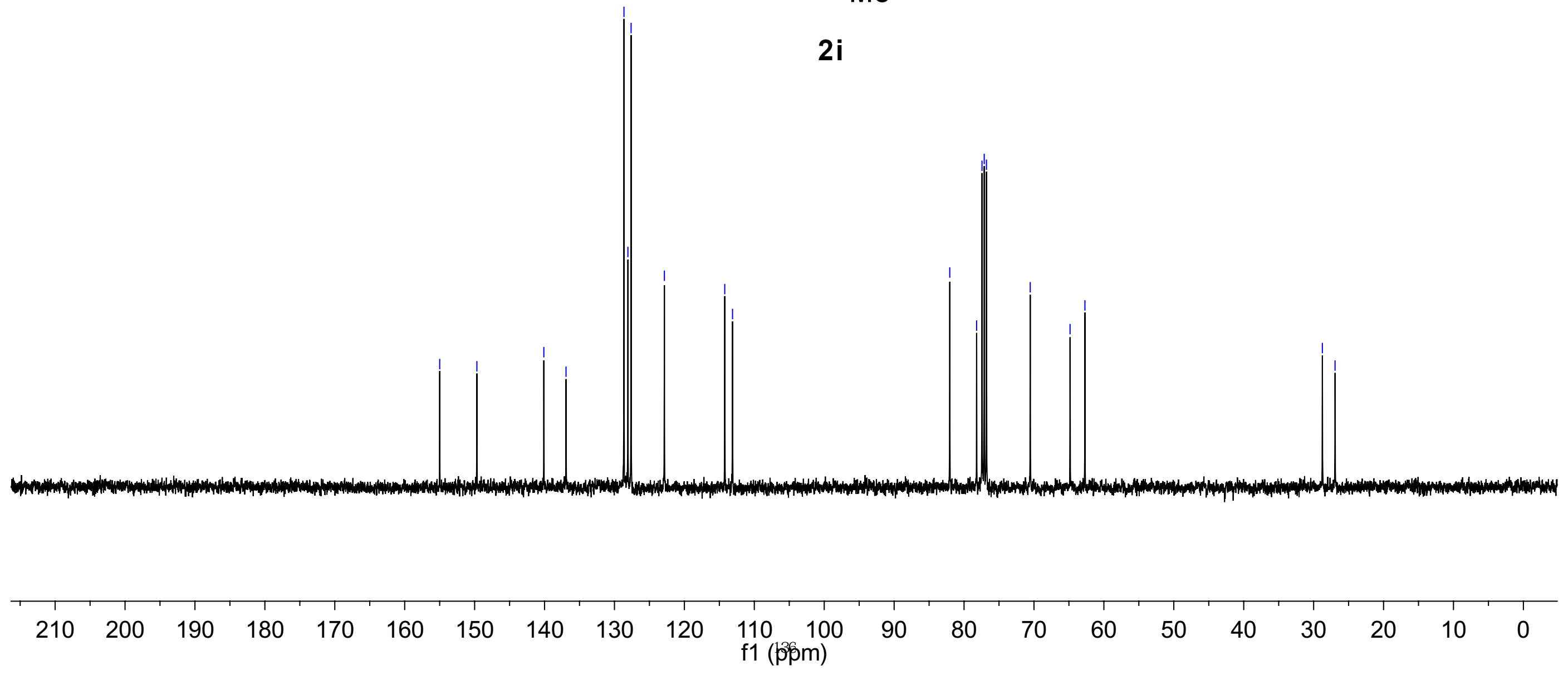




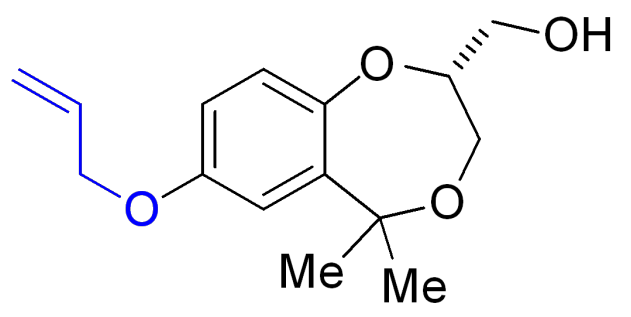

2j

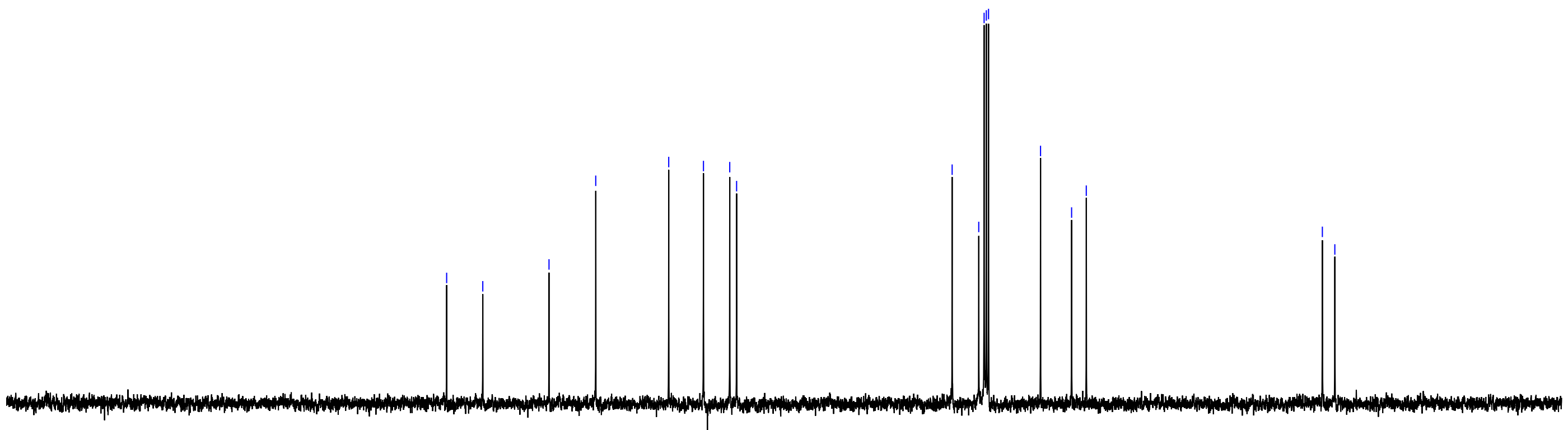

$\begin{array}{llllllllllllllllllllll}210 & 200 & 190 & 180 & 170 & 160 & 150 & 140 & 130 & 120 & \begin{array}{c}110 \\ \mathrm{f} 1(3 \mathrm{~s} p \mathrm{~m})\end{array} & 90 & 80 & 70 & 60 & 50 & 40 & 30 & 20 & 10 & 0\end{array}$




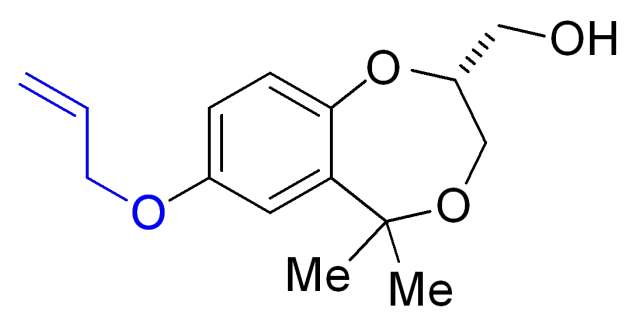

2j

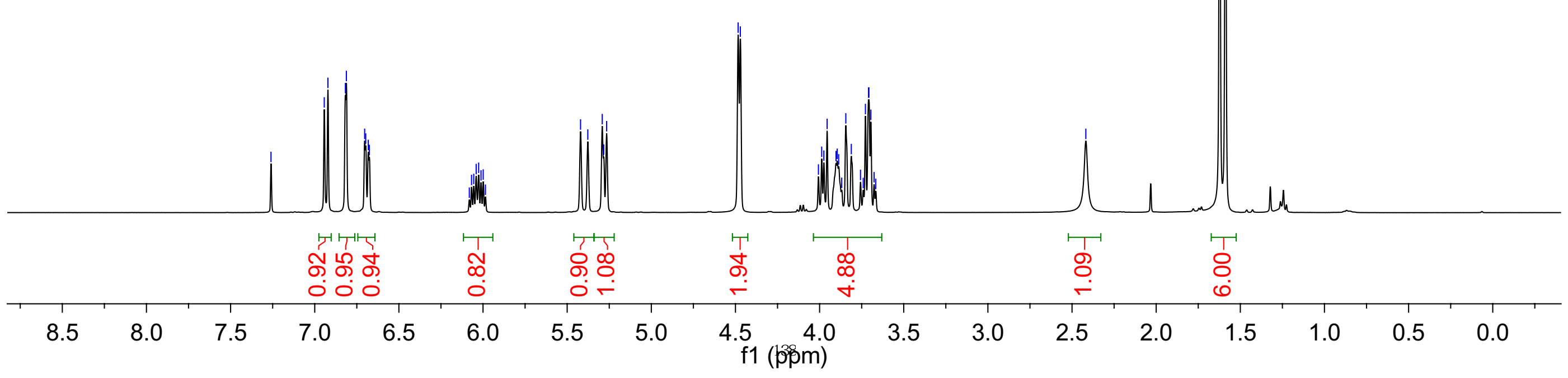




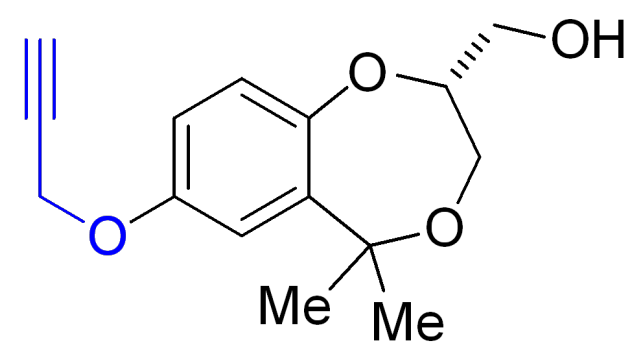

\section{2k}

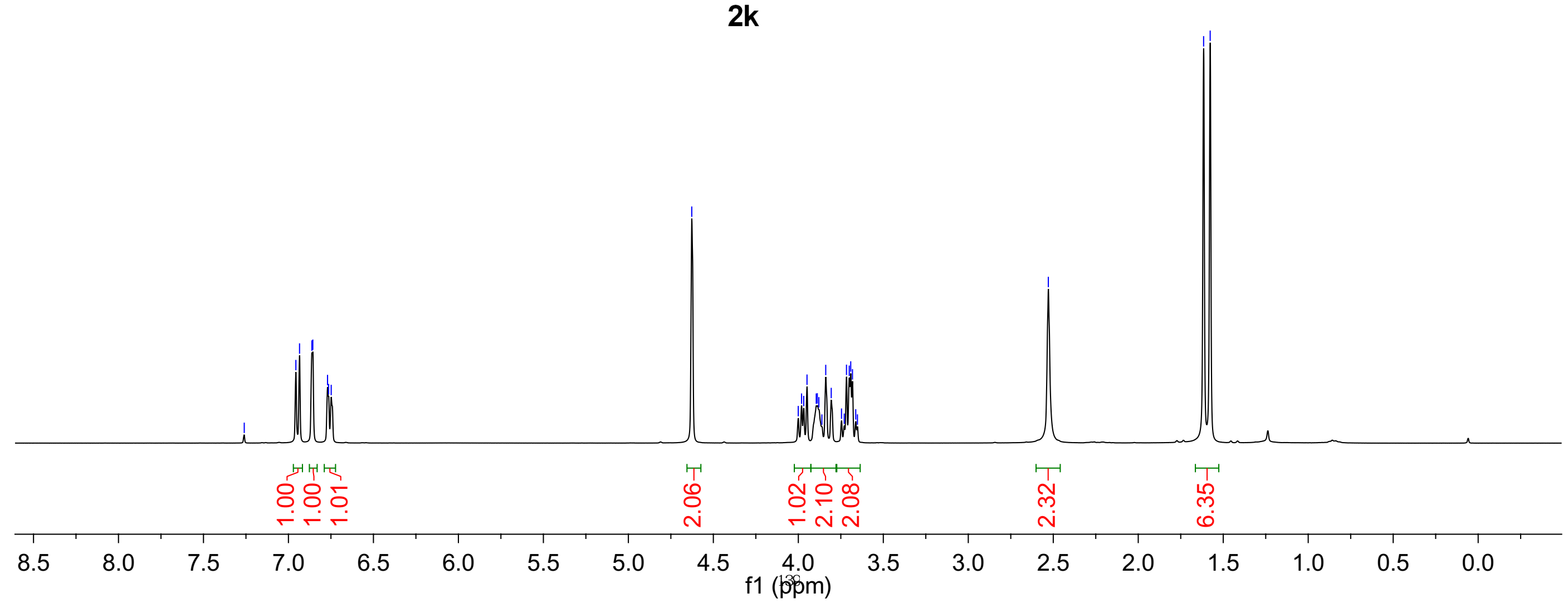


<smiles>C#CCOc1ccc2c(c1)C(C)(C)OC[C@H](CO)O2</smiles>

2k

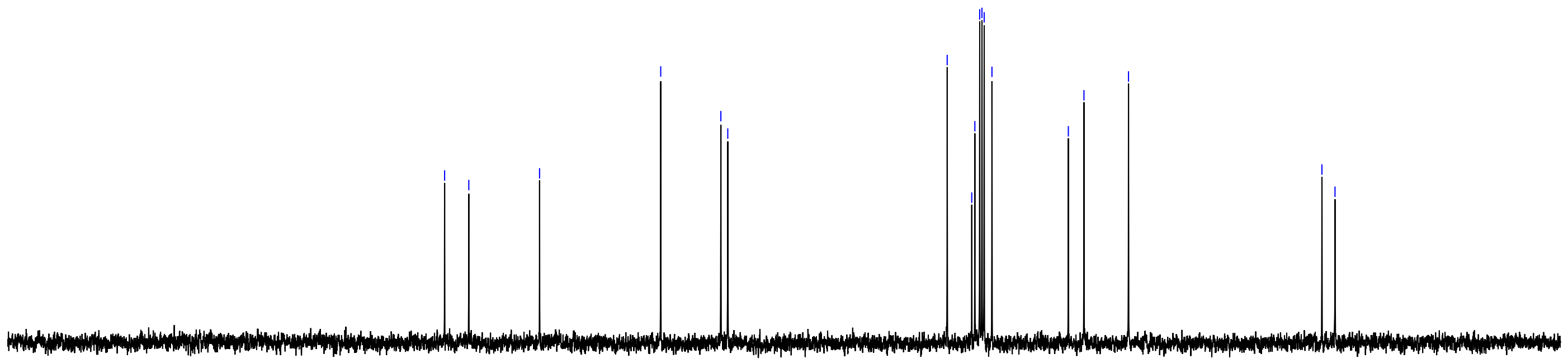

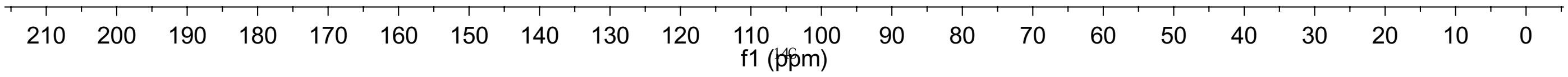



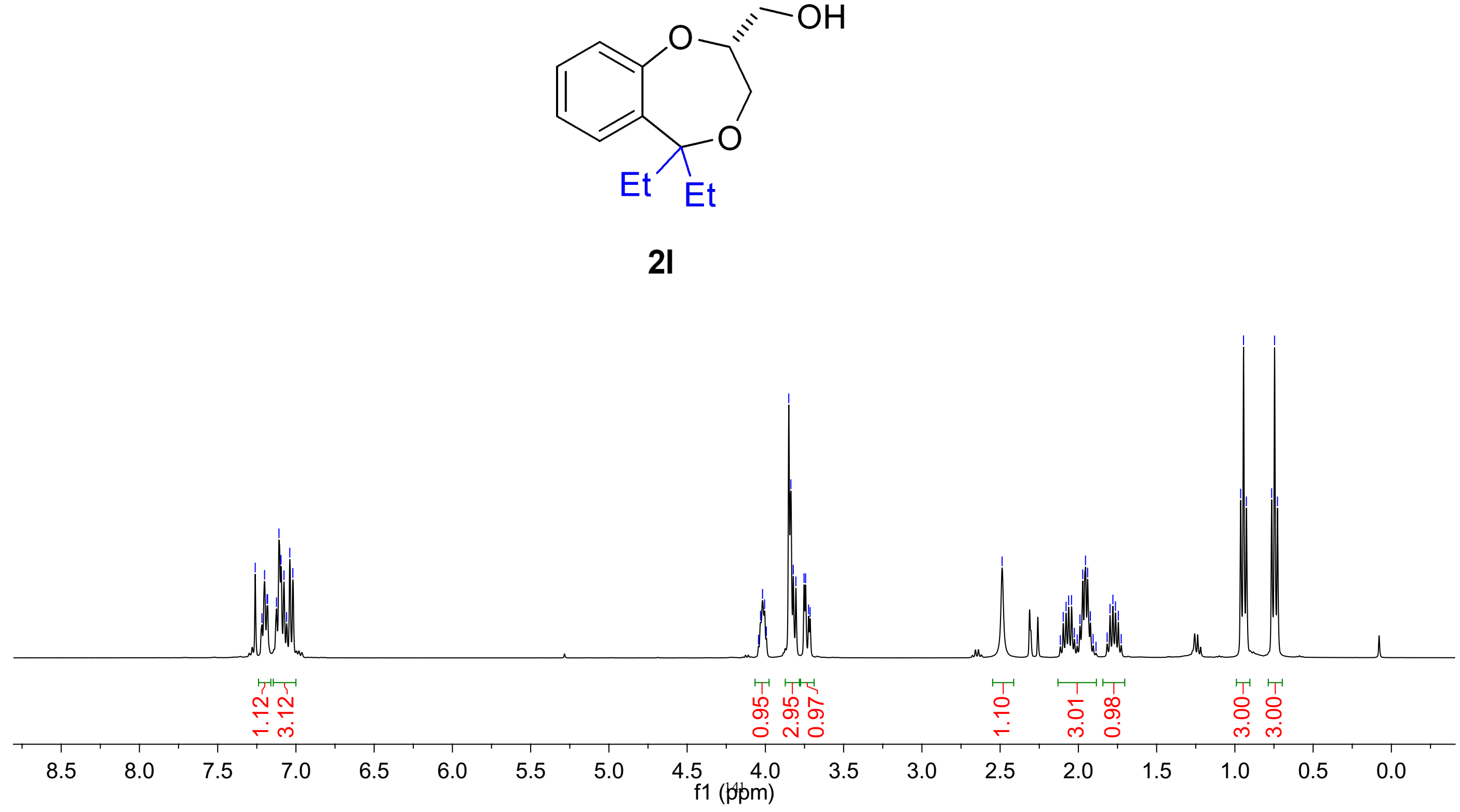


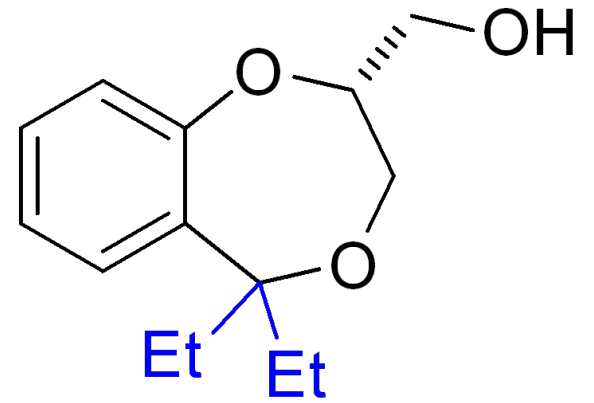

21

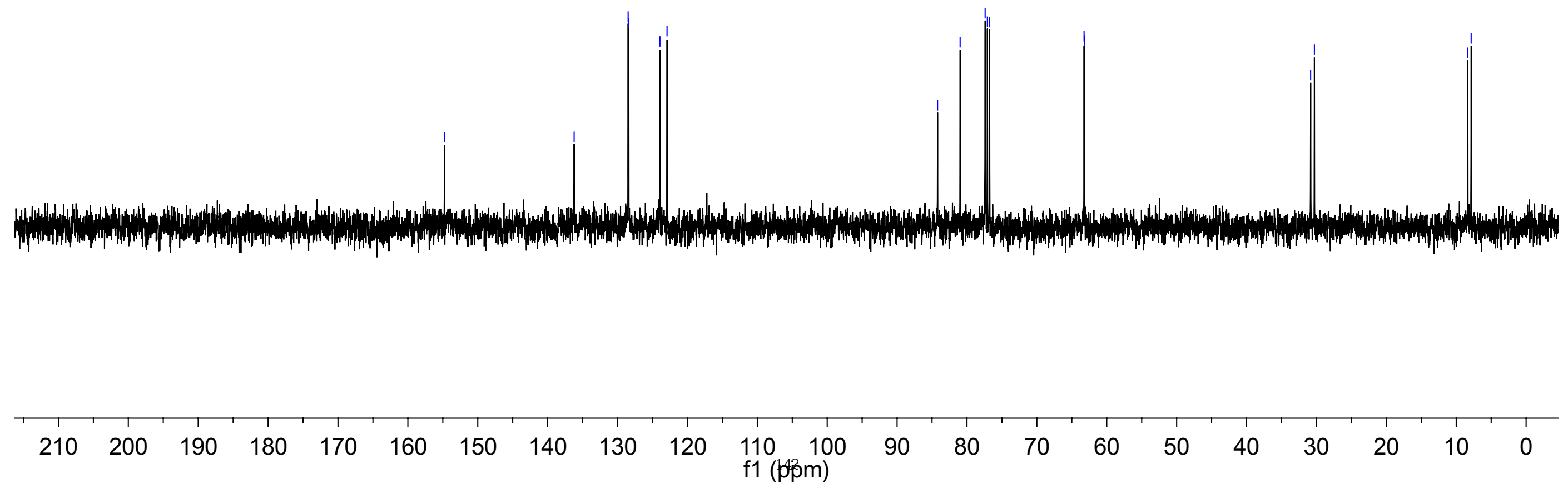




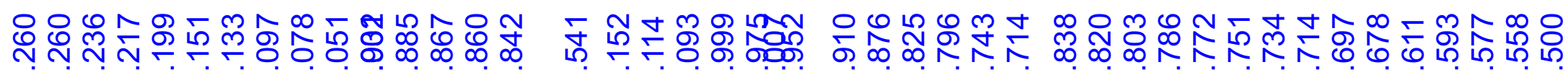

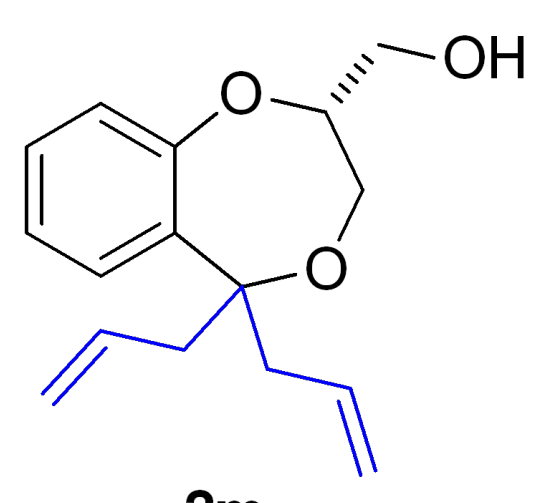

\section{$2 m$}

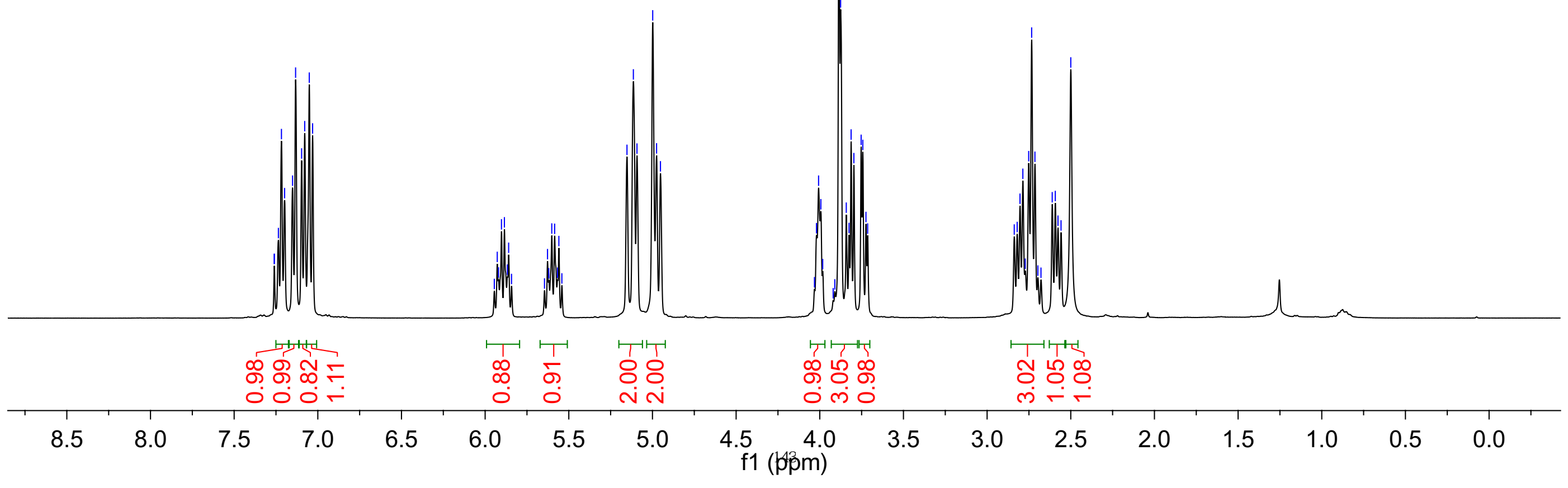




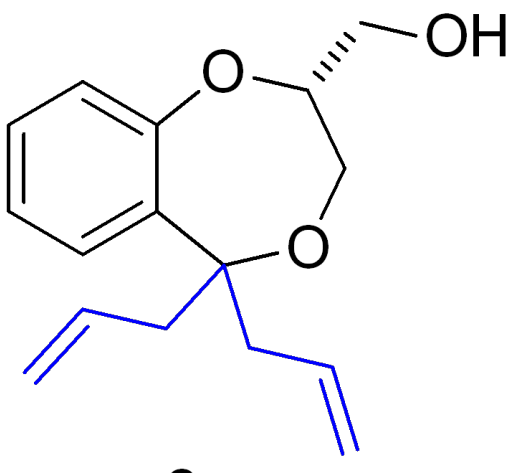

$2 m$

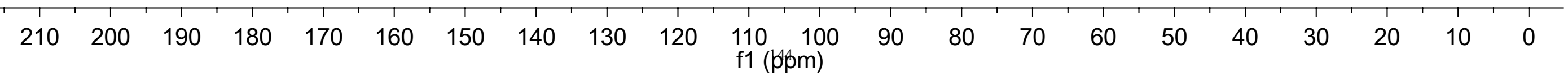




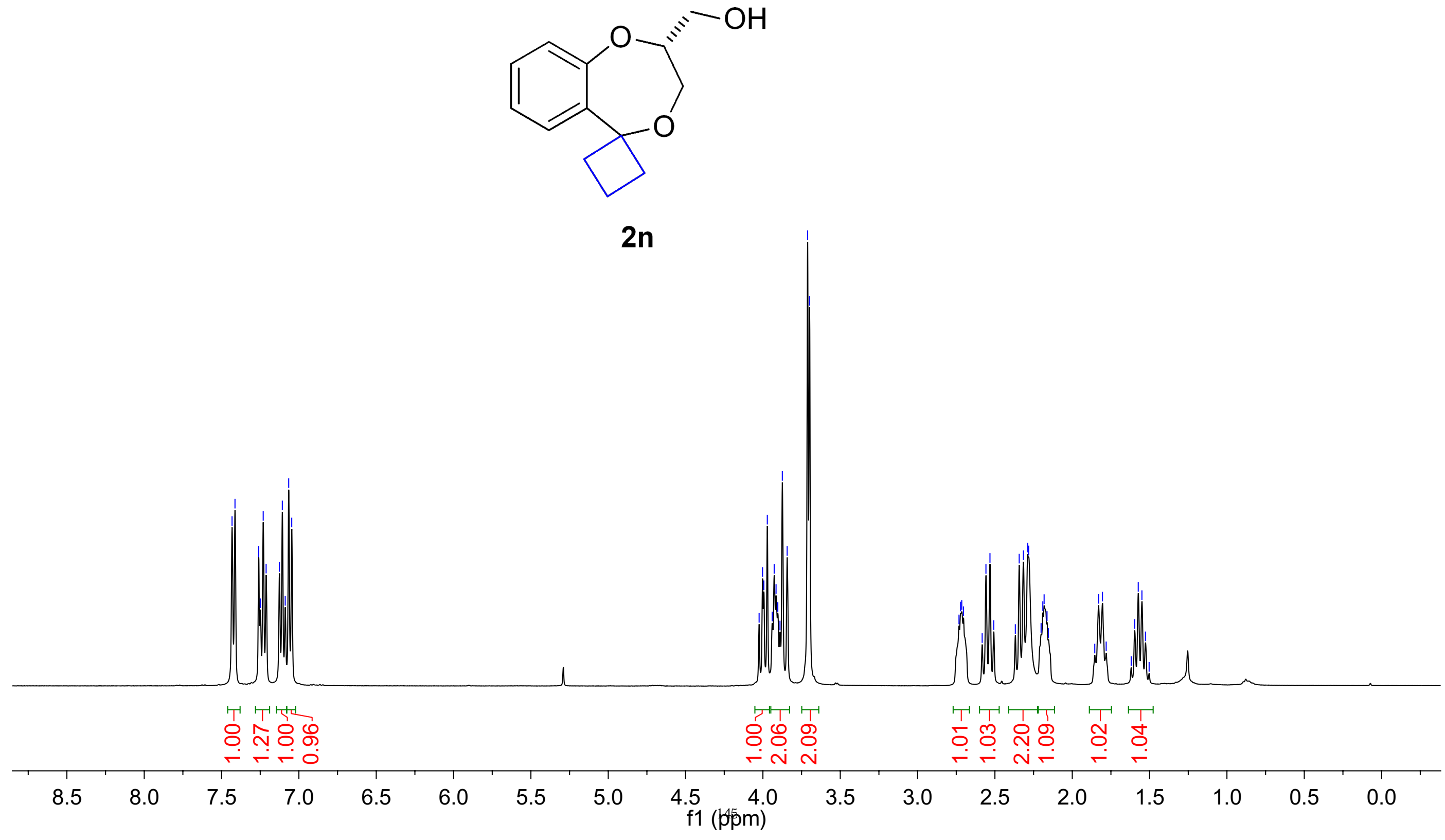




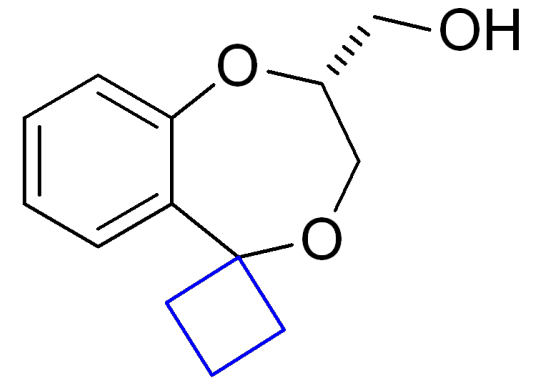

$2 n$

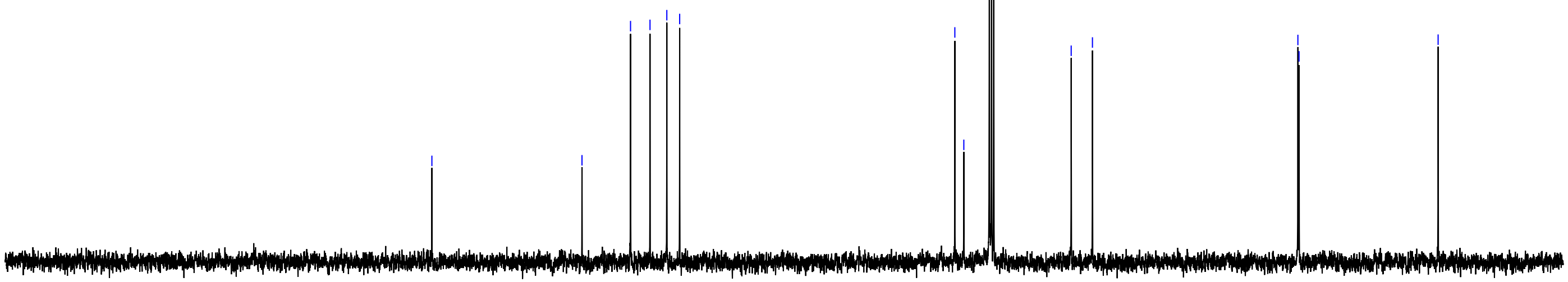



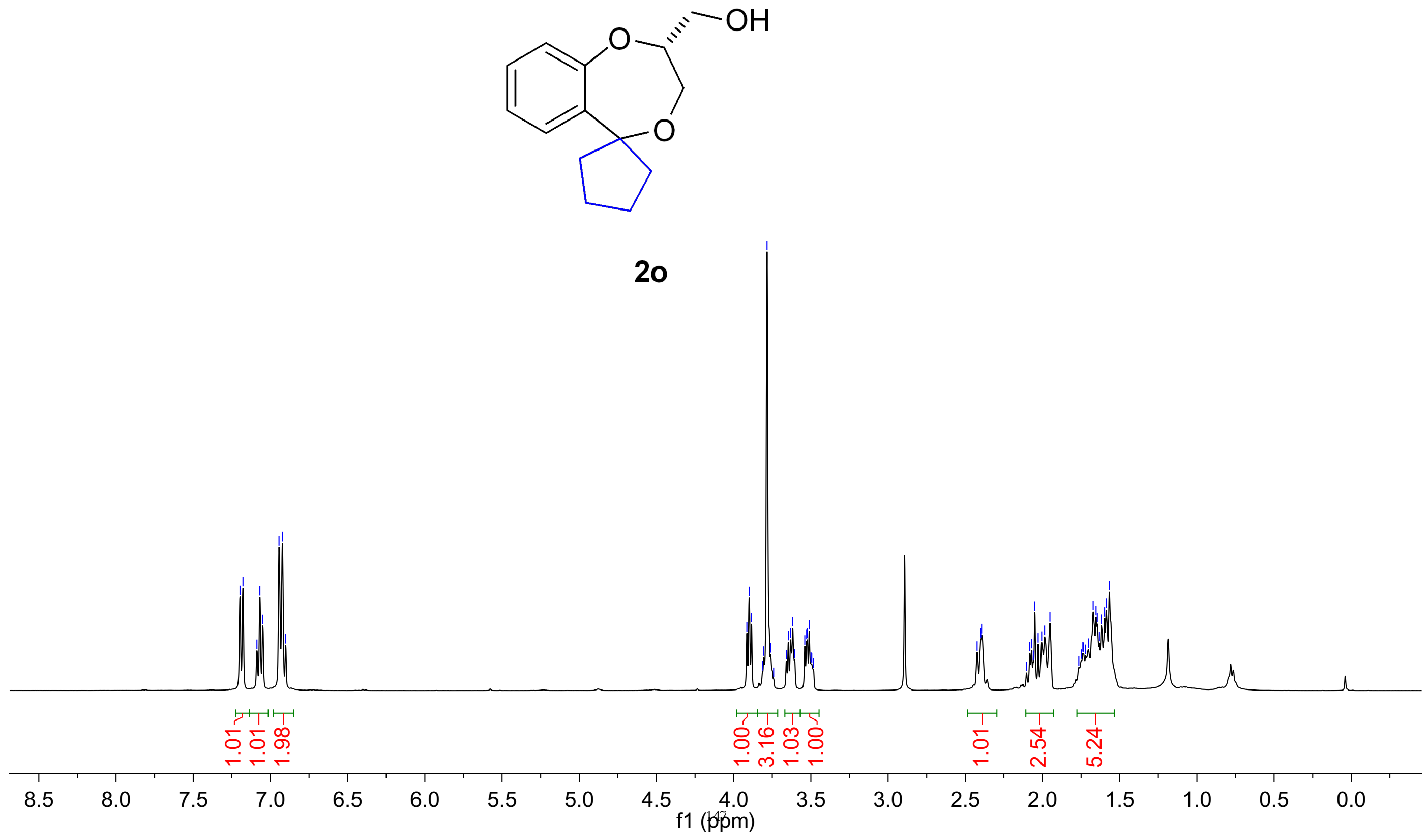


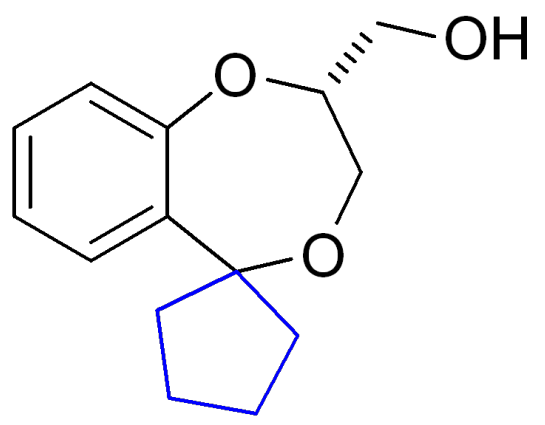

20 


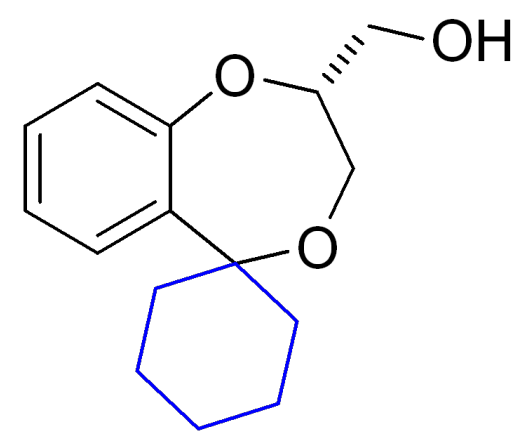

$2 p$

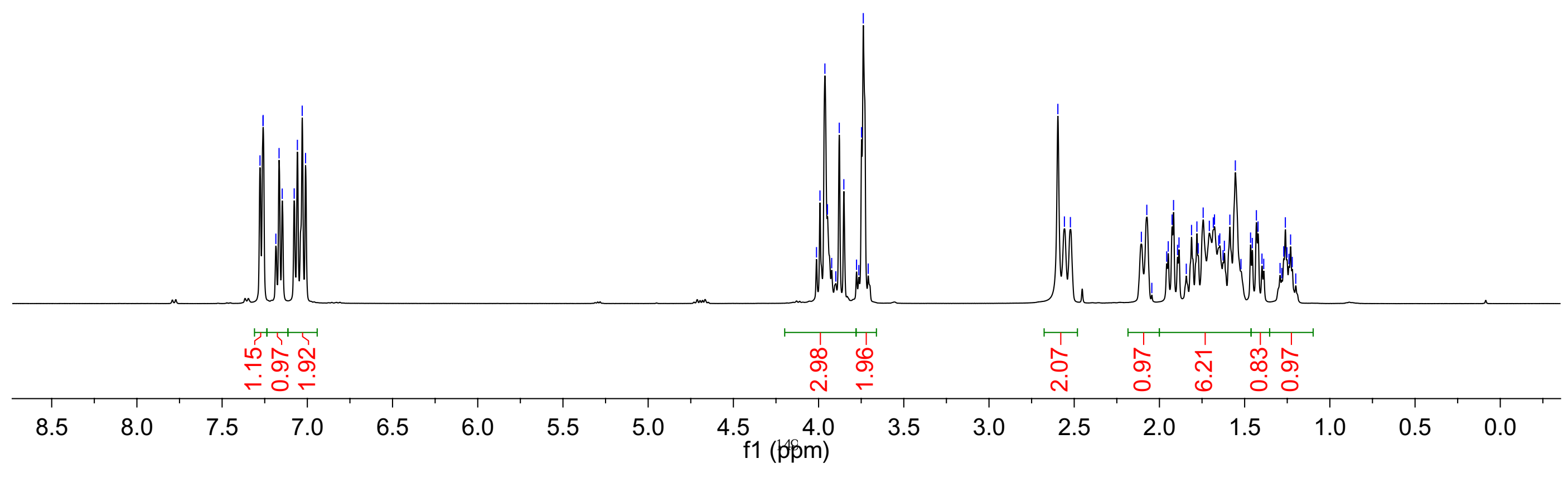




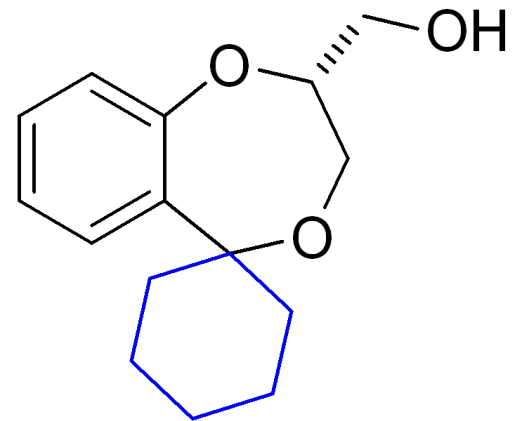

$2 p$

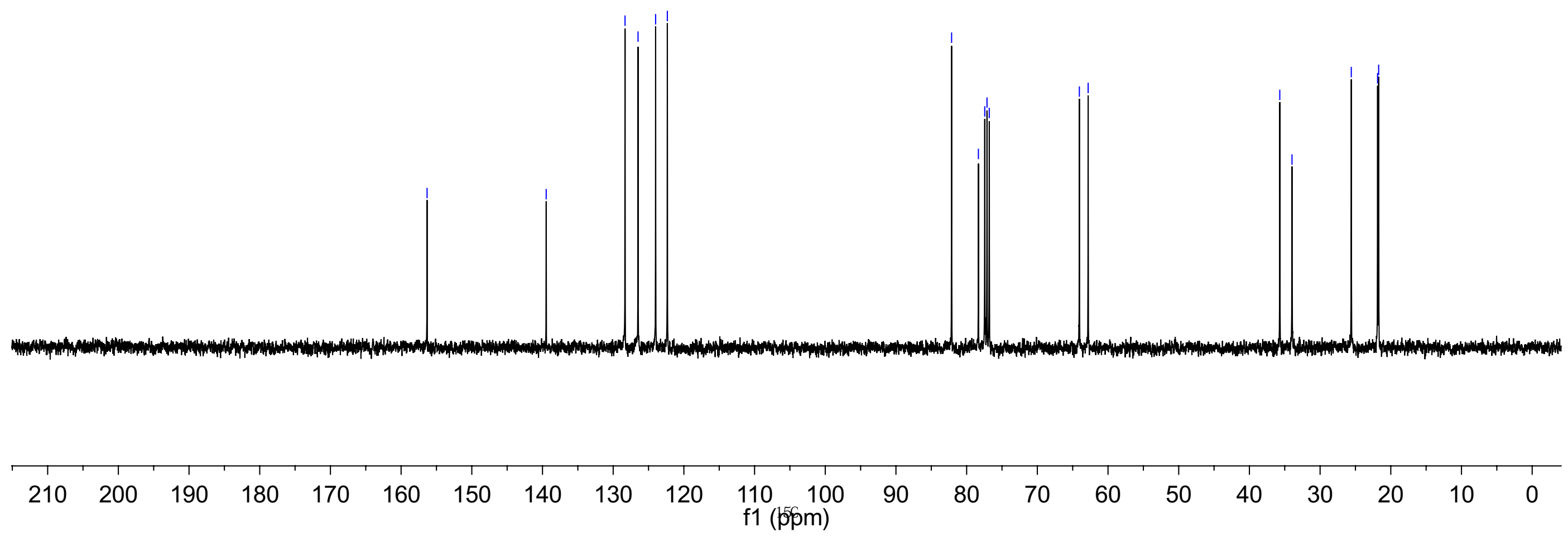




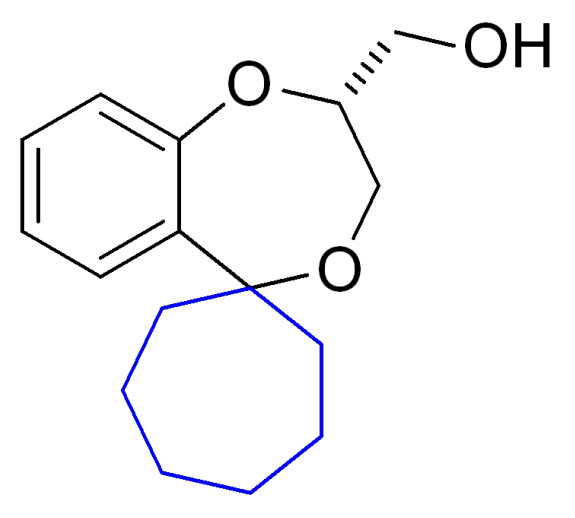

$2 q$

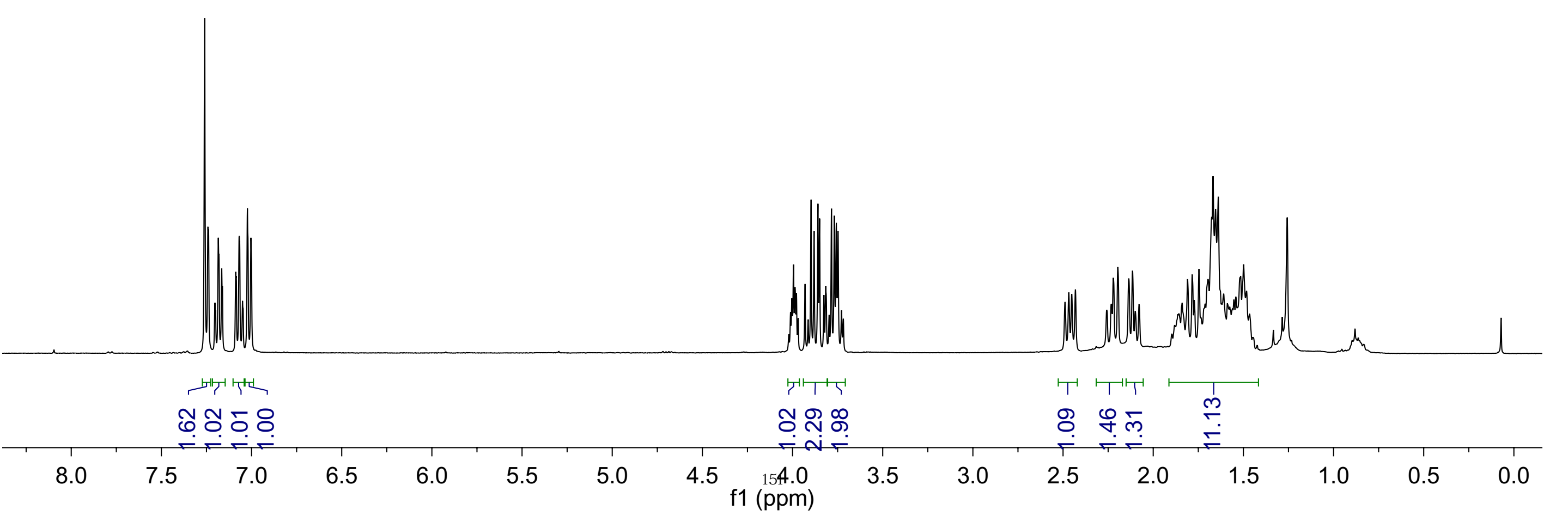




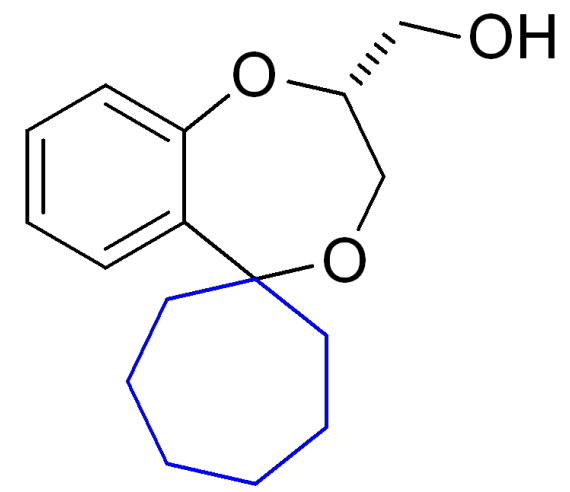

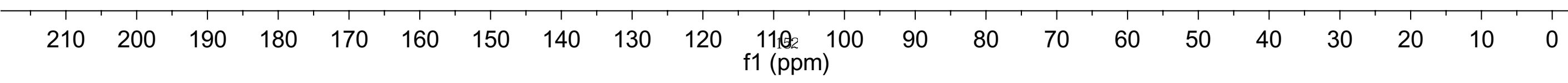



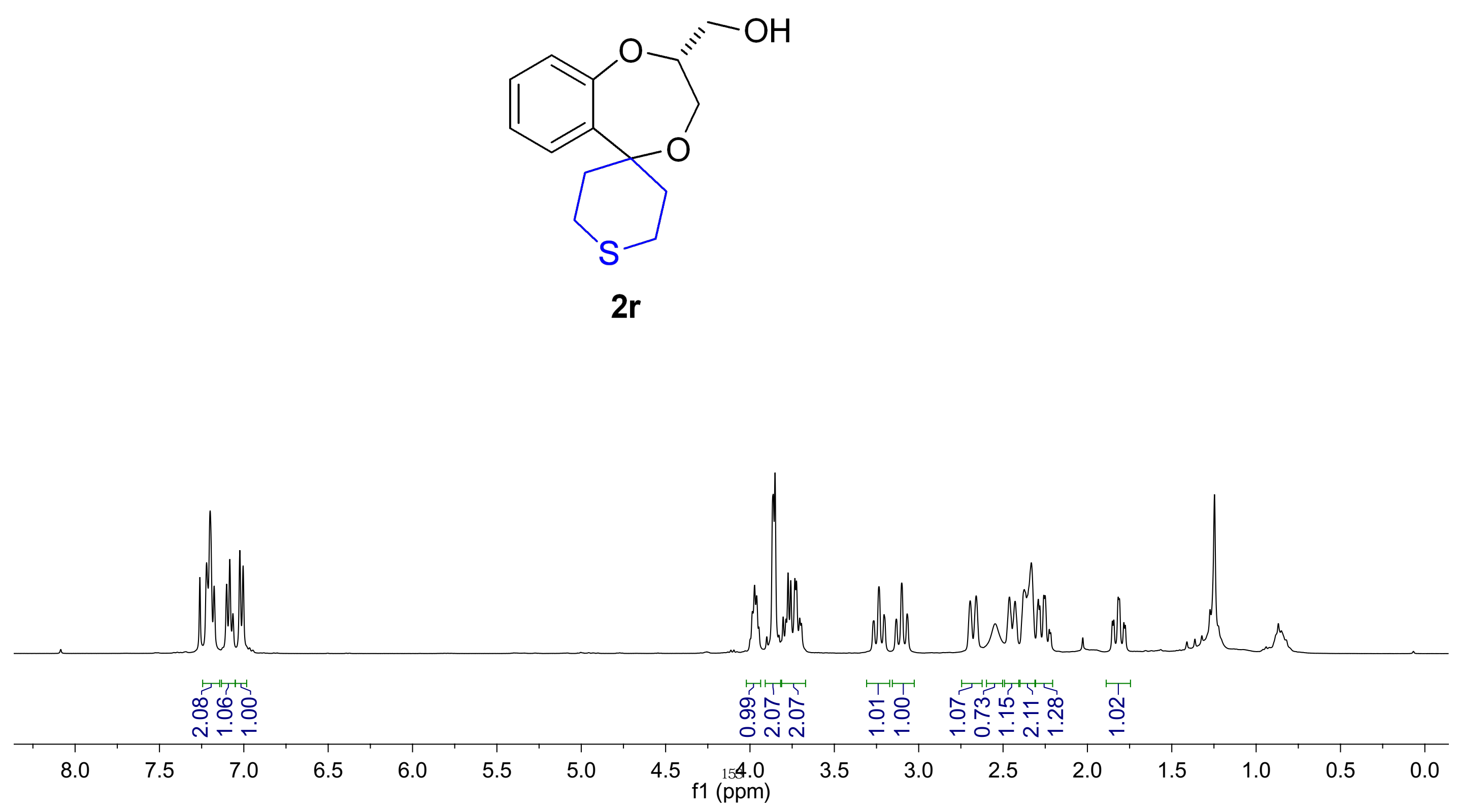


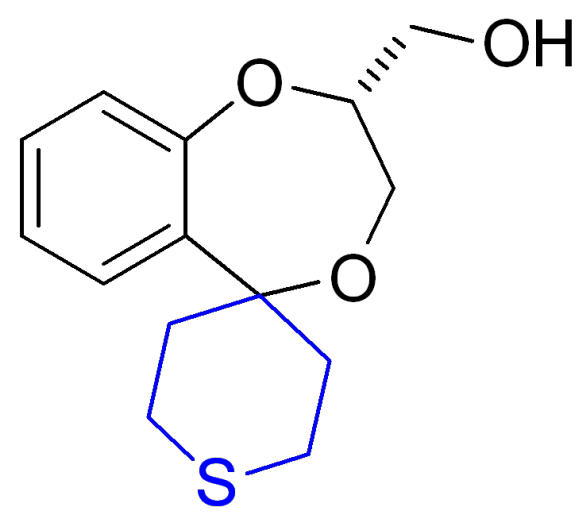

\section{$2 r$}

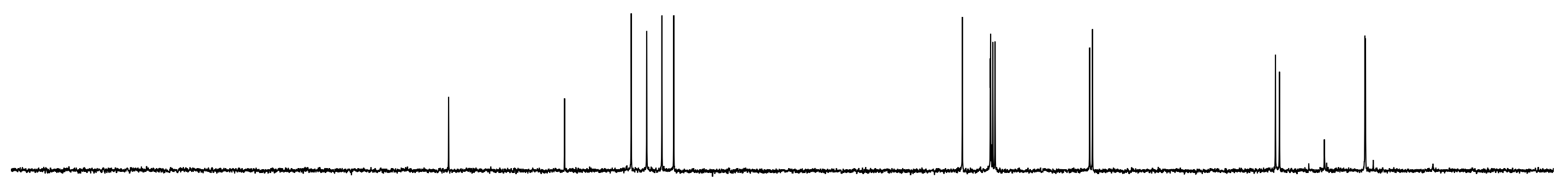

\begin{tabular}{|c|c|c|c|c|c|c|c|c|c|c|c|c|c|c|c|c|c|c|c|}
\hline 0 & 200 & 190 & 180 & 170 & 160 & 150 & 140 & 130 & 120 & $\begin{array}{l}110 \overline{10} 100 \\
\text { f1 (ppm) }\end{array}$ & 90 & 80 & 70 & 60 & 50 & 40 & 30 & 20 & 10 \\
\hline
\end{tabular}




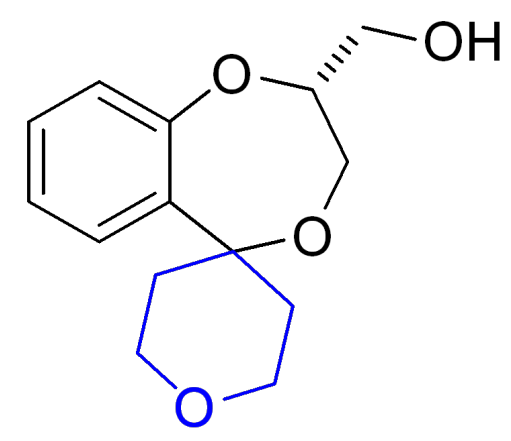

2s

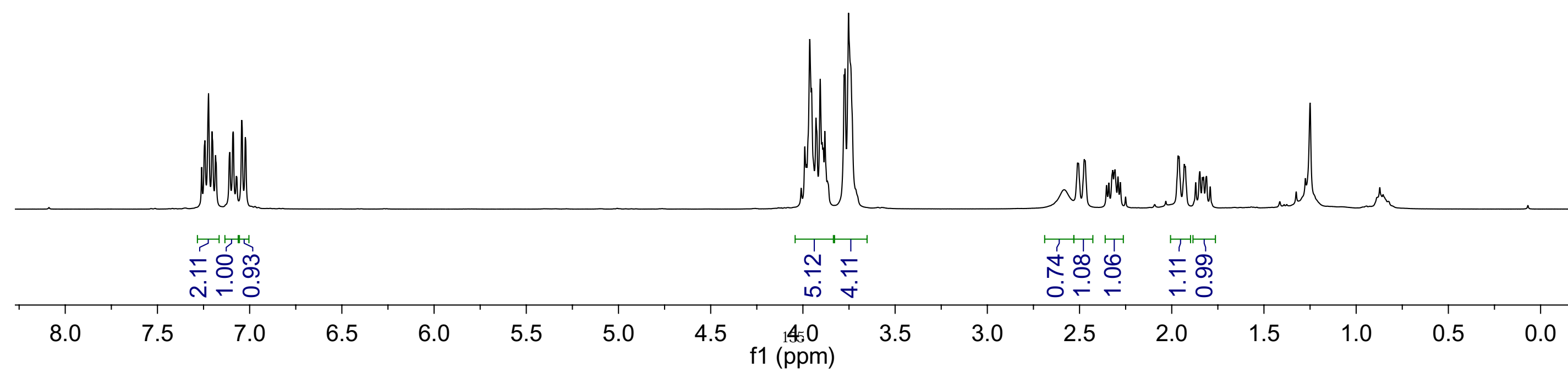




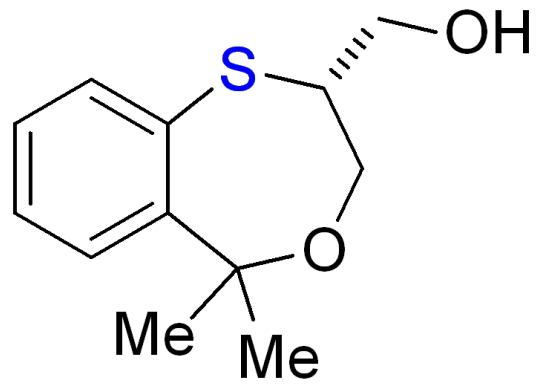

\section{$2 b-S$}

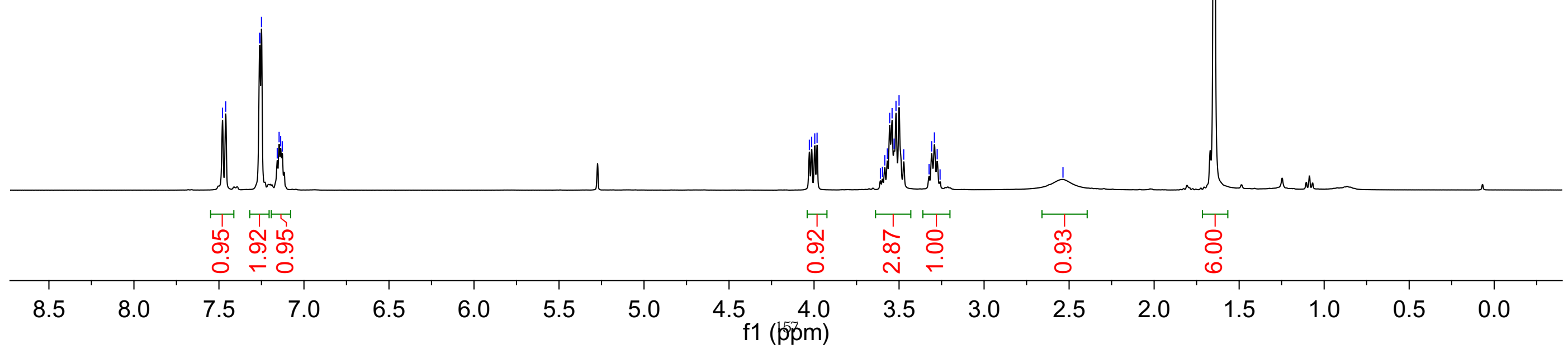




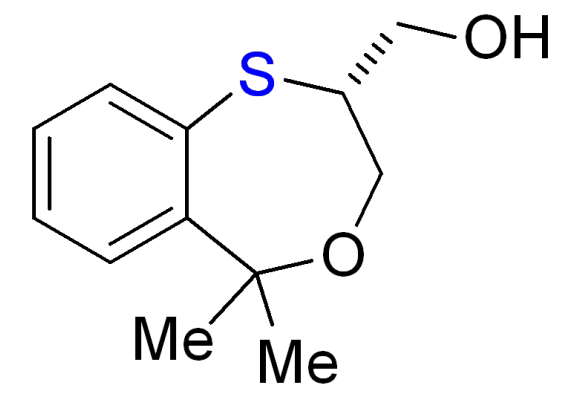

$2 b-S$

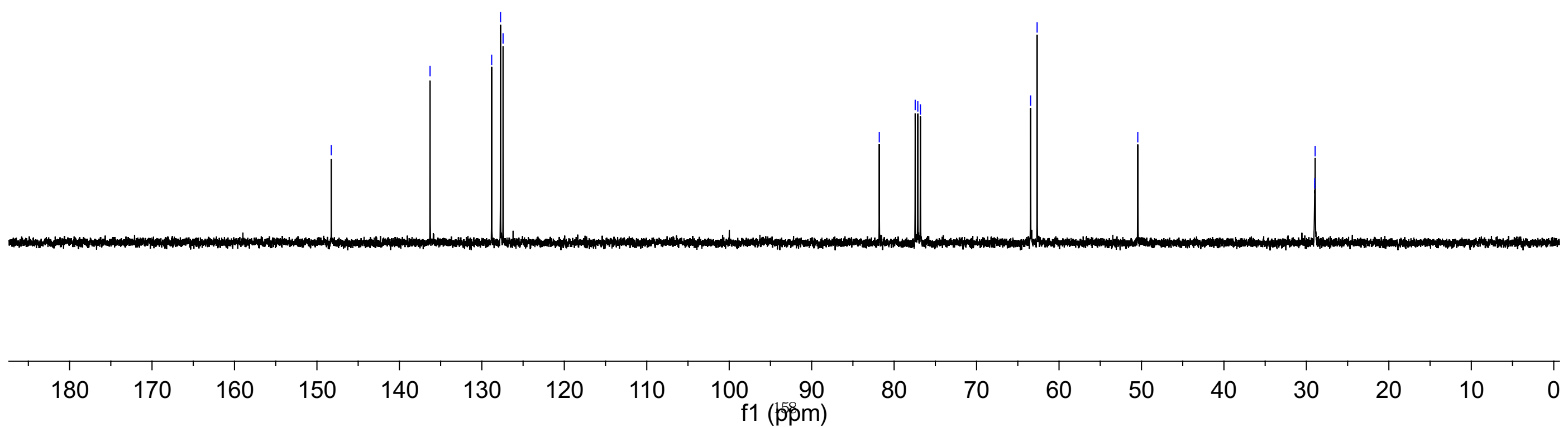




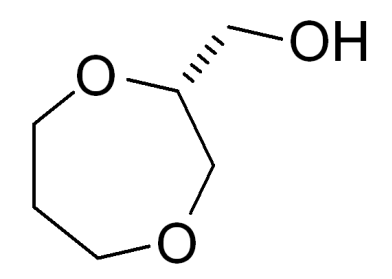

4

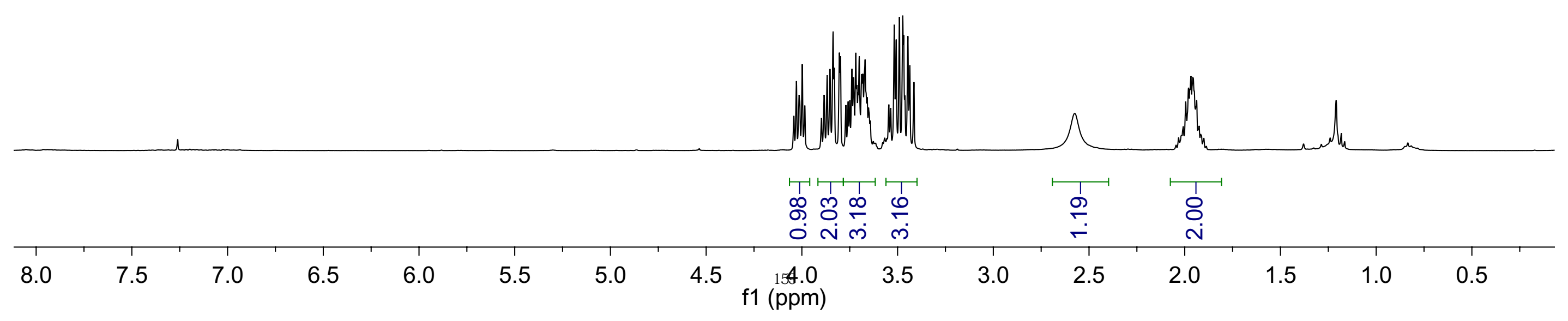



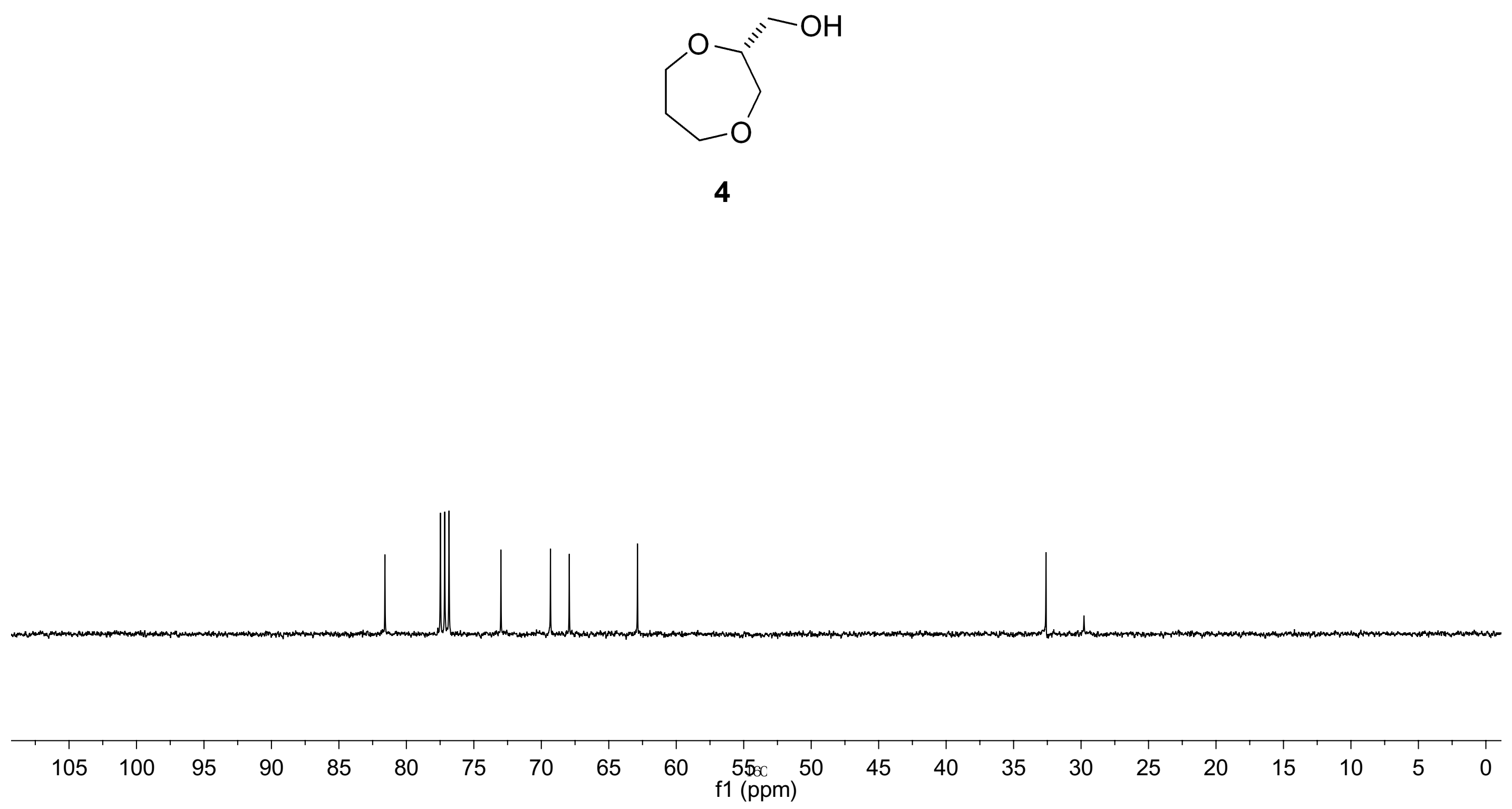


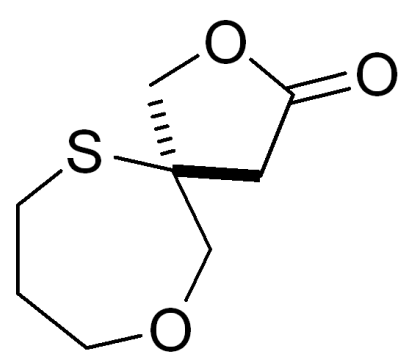

6

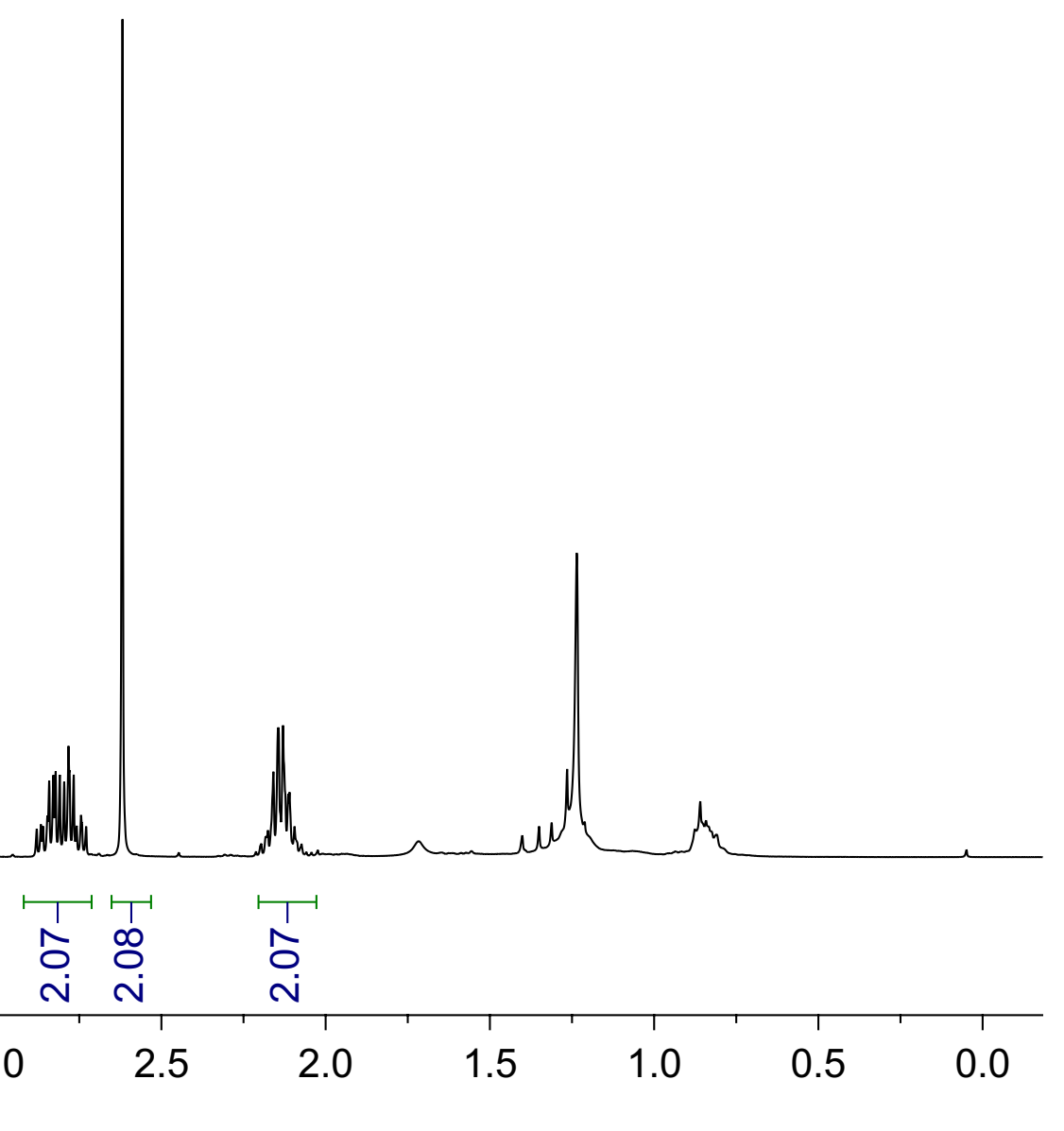




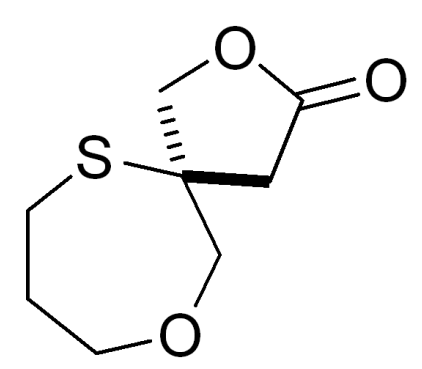

6

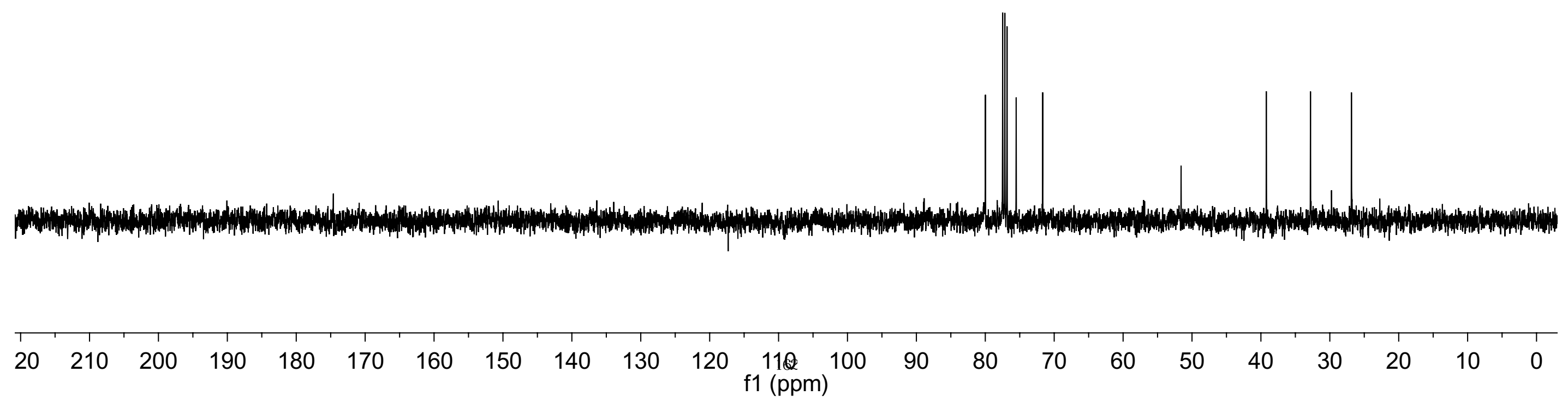




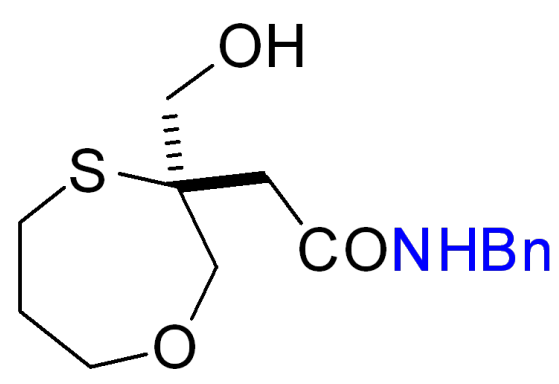

7

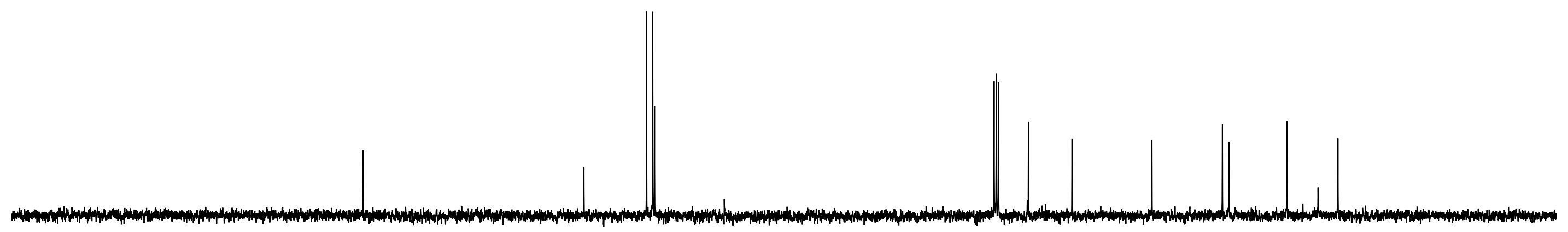

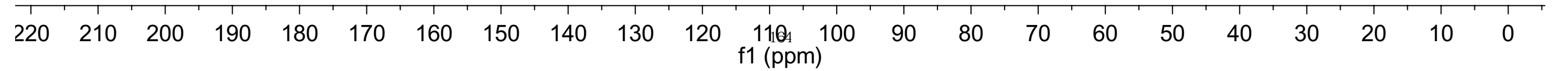




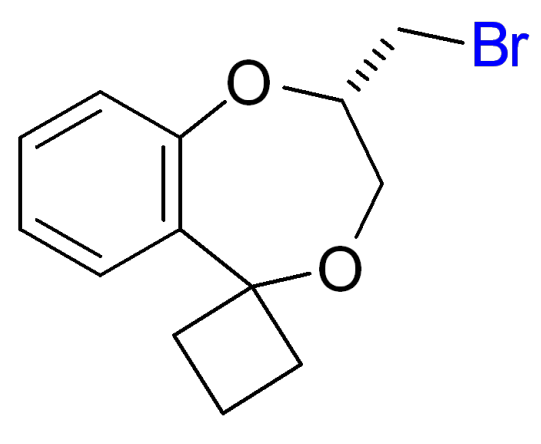

8

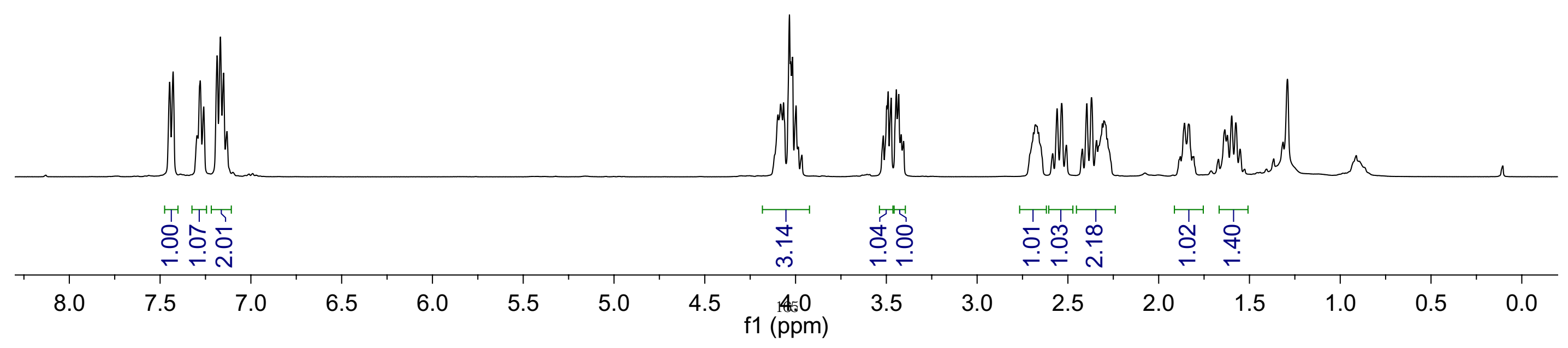




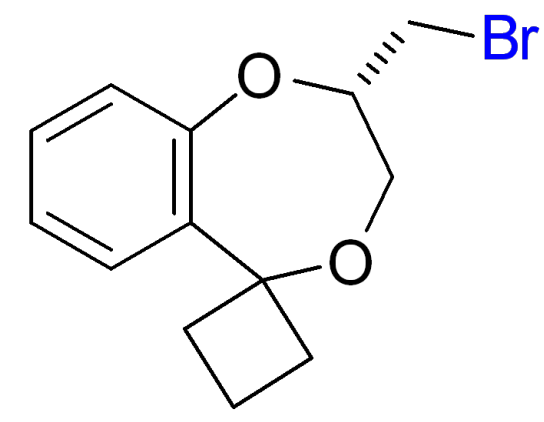

8

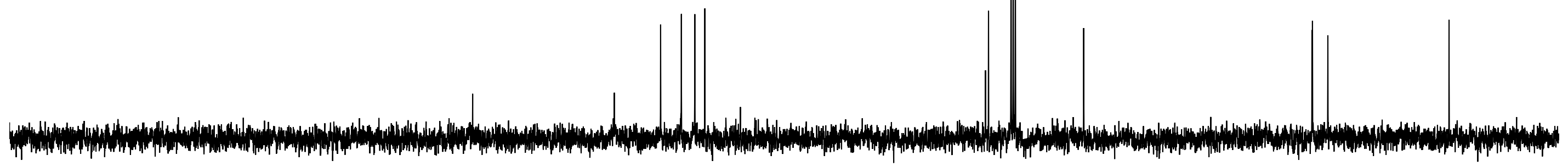

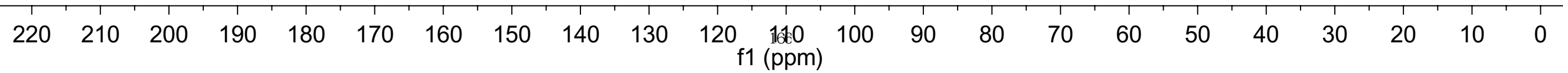




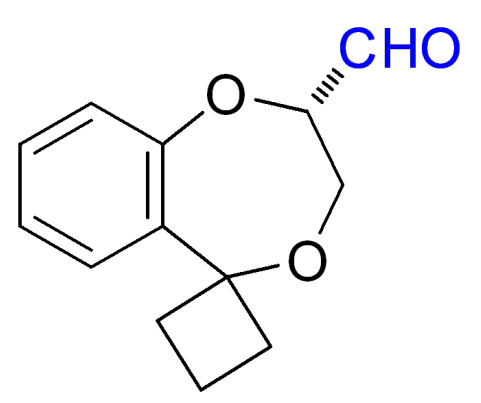

9

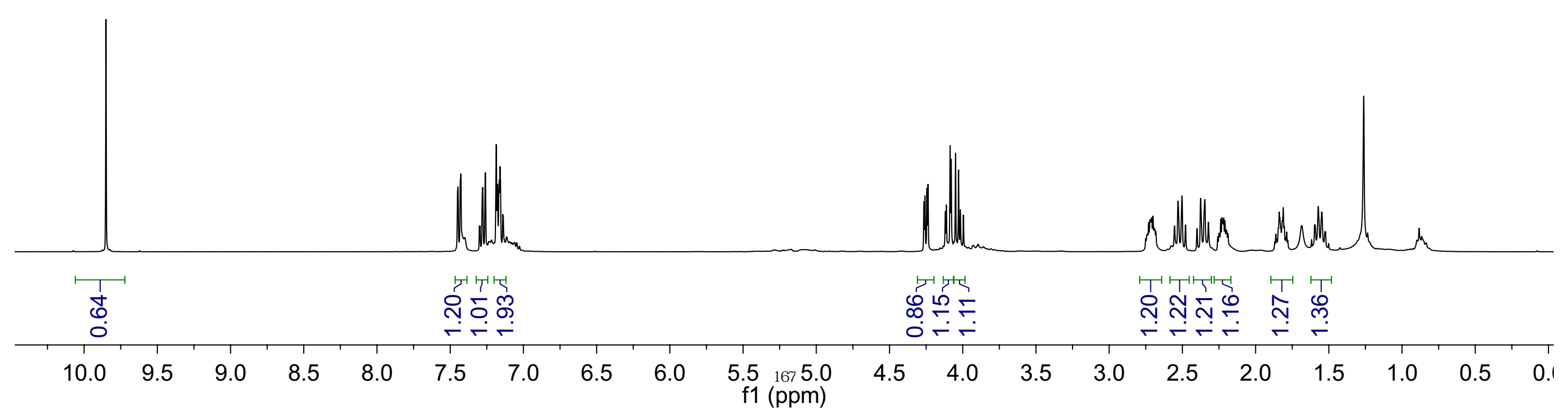




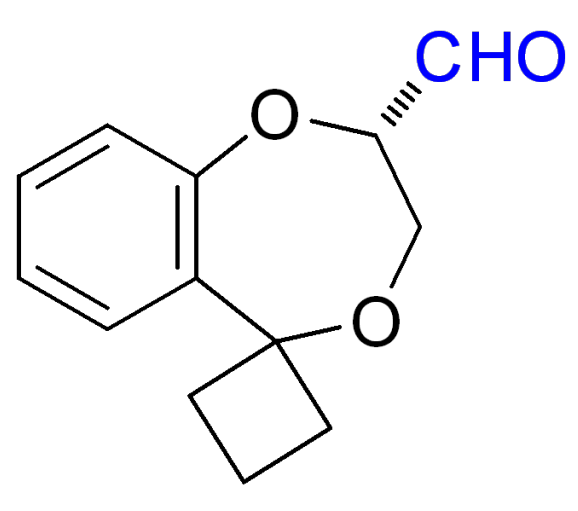

9

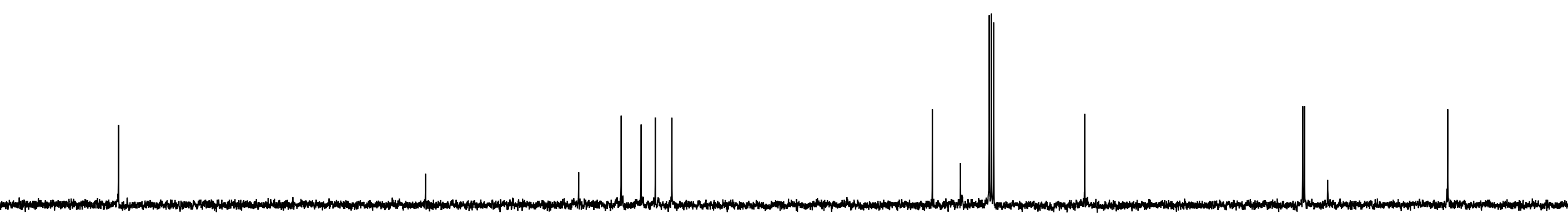

$\begin{array}{llllllllllllllllllllllll}210 & 200 & 190 & 180 & 170 & 160 & 150 & 140 & 130 & 120 & \begin{array}{c}110_{168} 100 \\ \mathrm{f} 1(\mathrm{ppm})\end{array} & 90 & 80 & 70 & 60 & 50 & 40 & 30 & 20 & 10 & 0\end{array}$




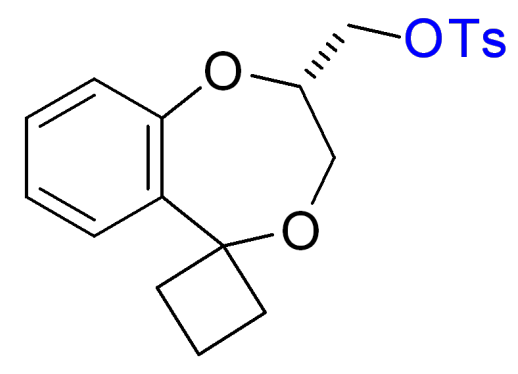

10

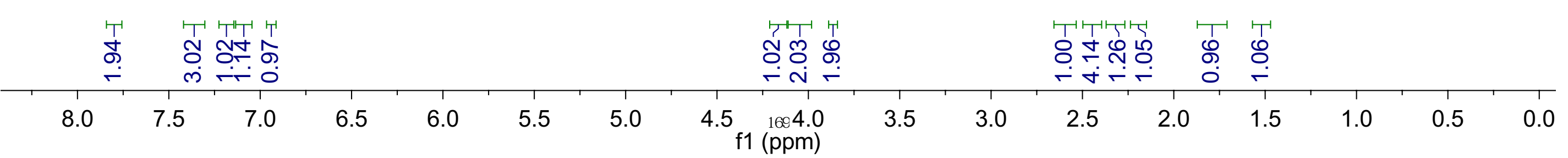



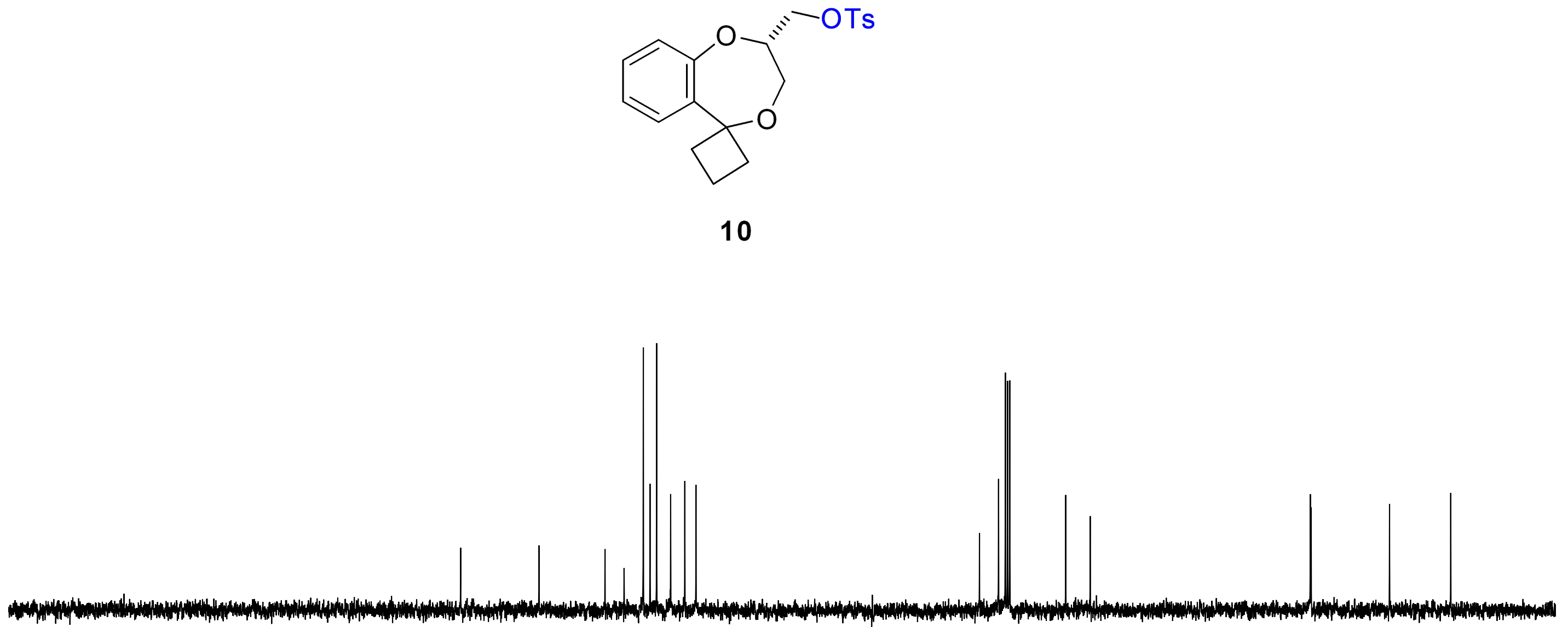

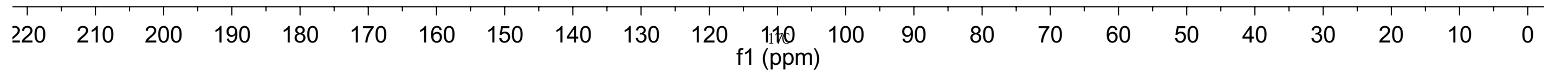




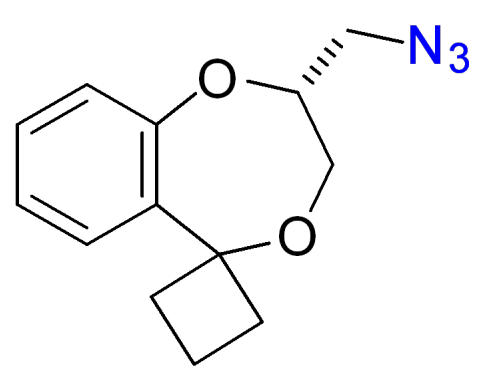

11

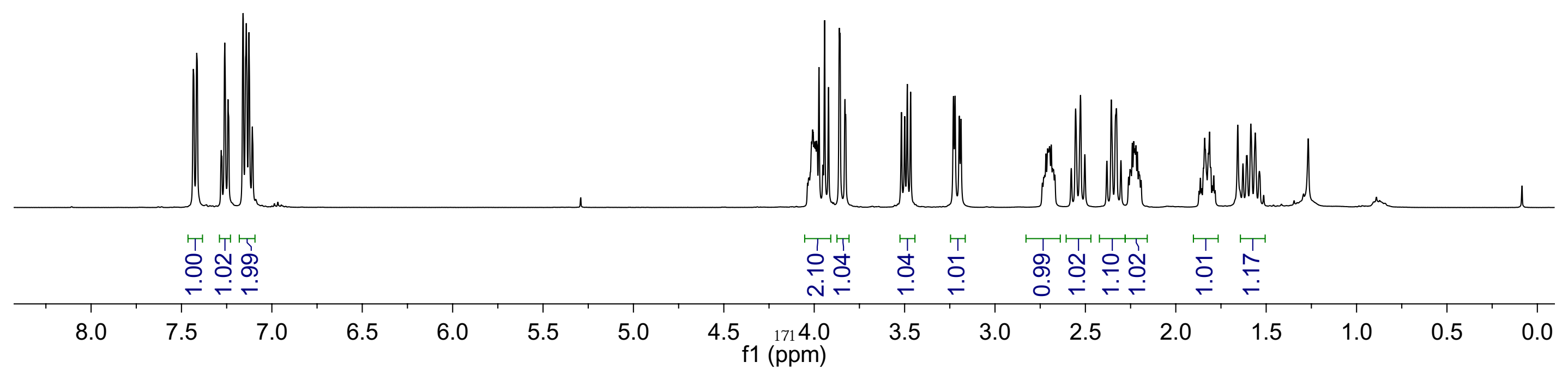



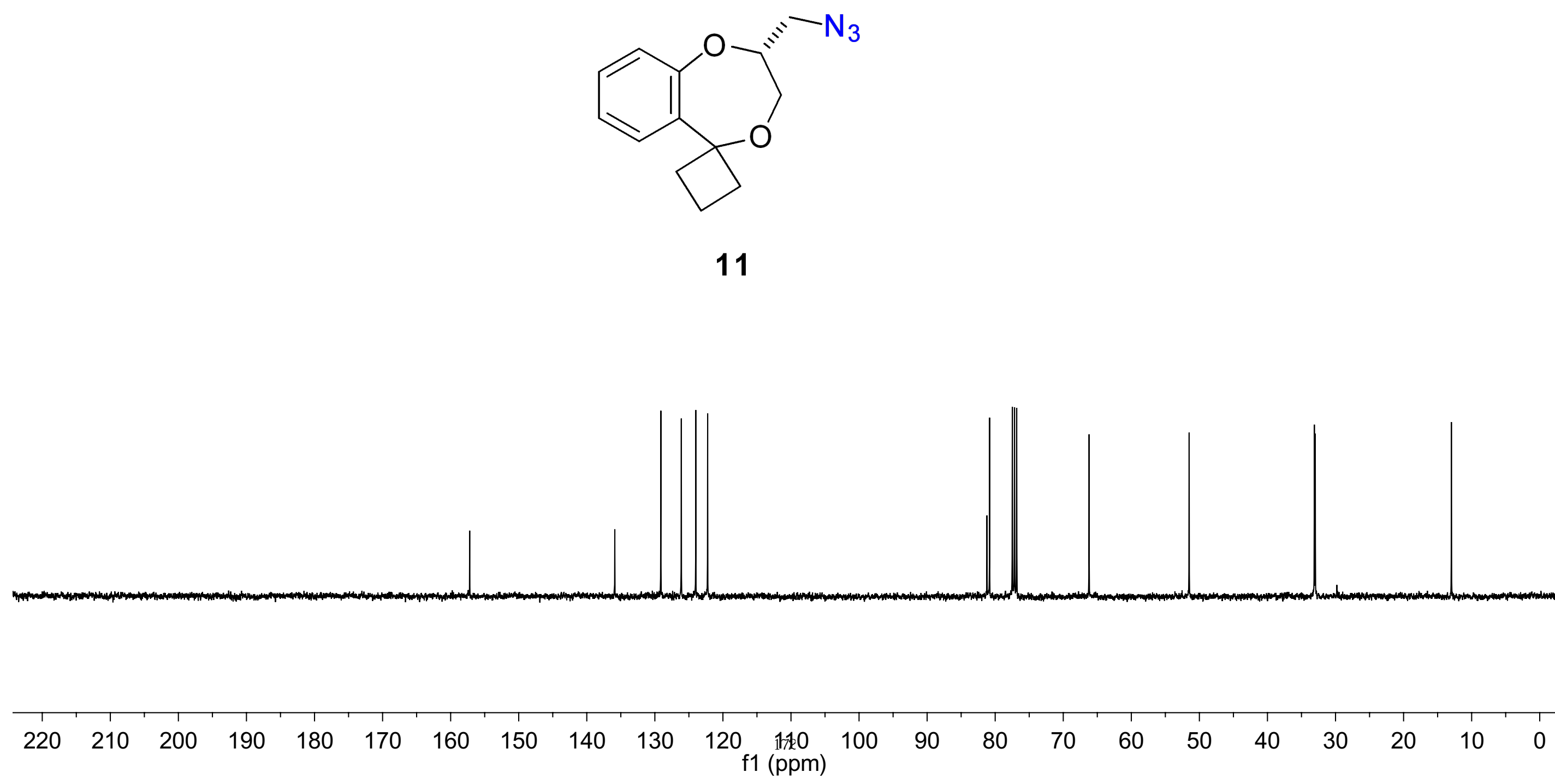


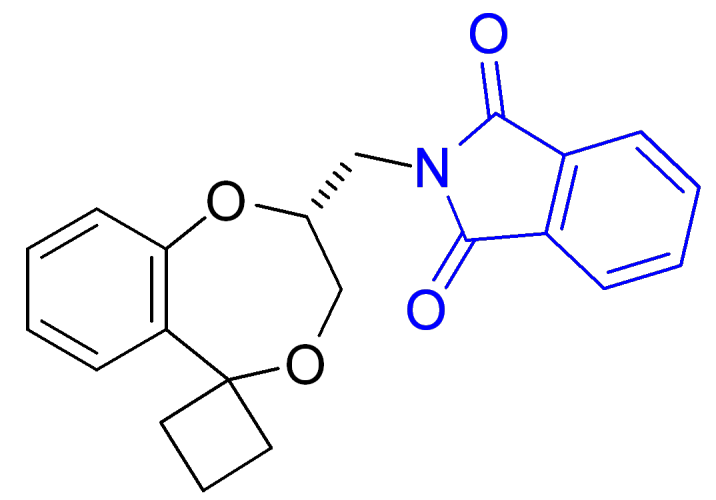

12

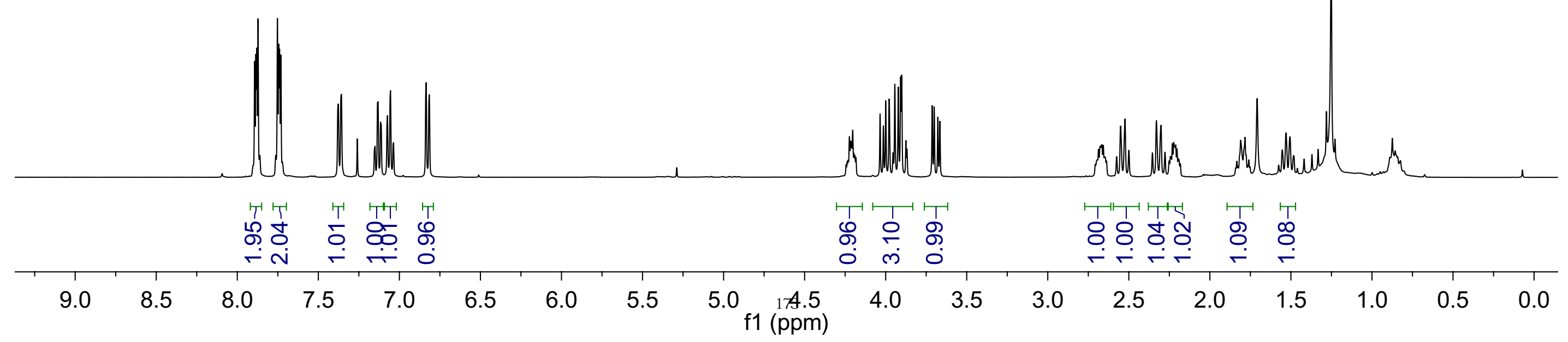




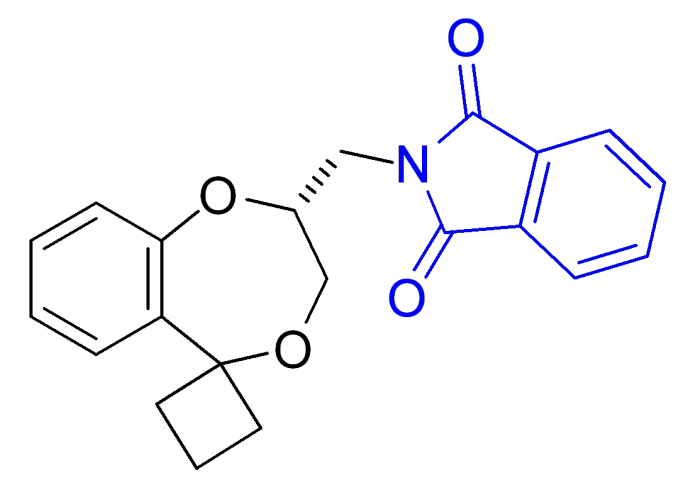

12
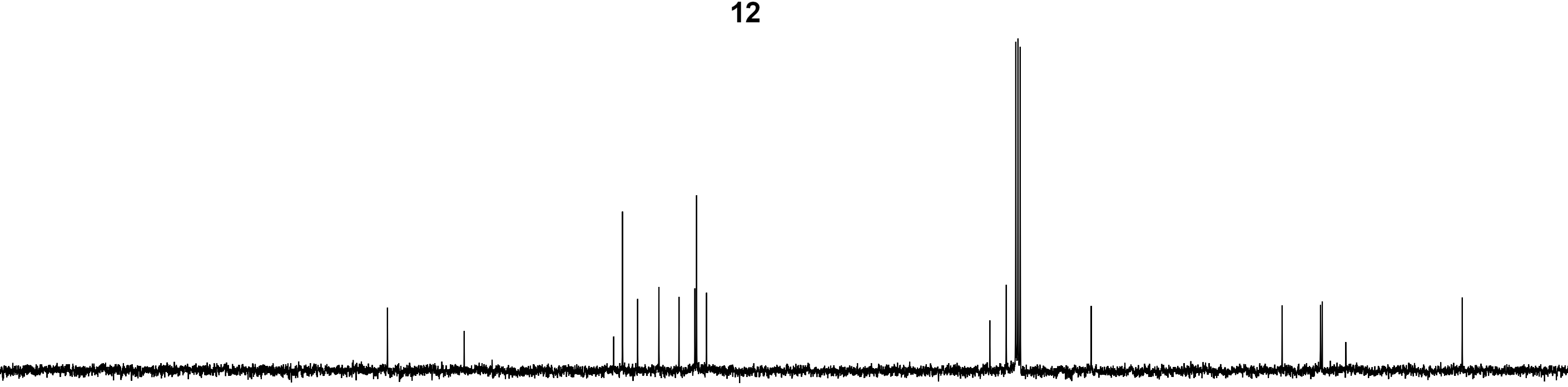


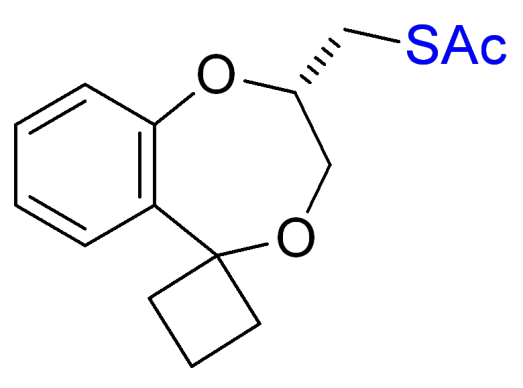

13

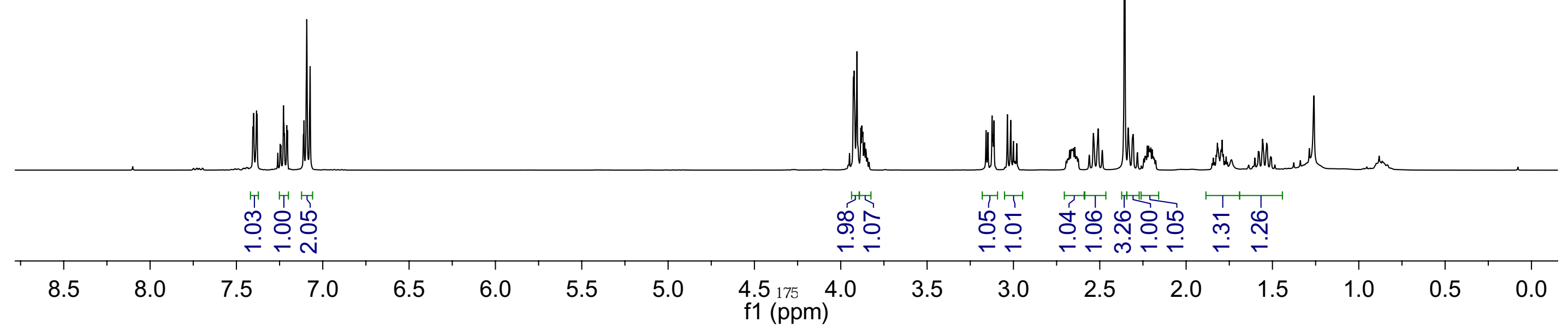




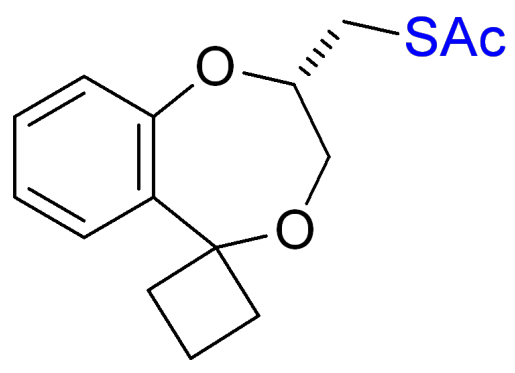

13

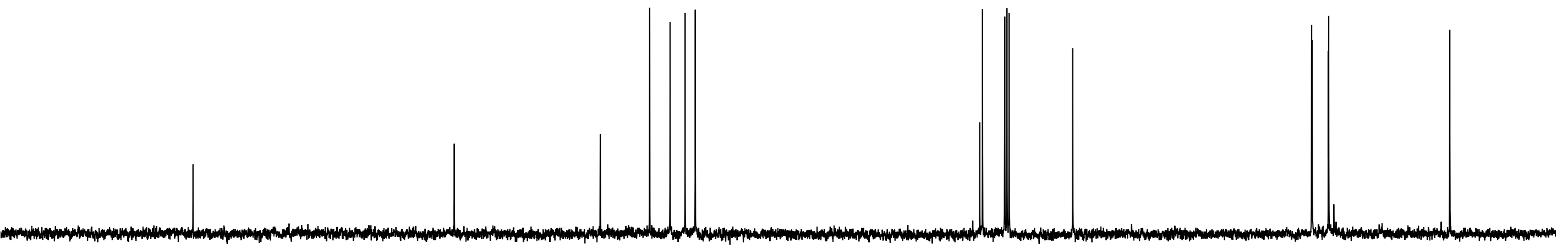

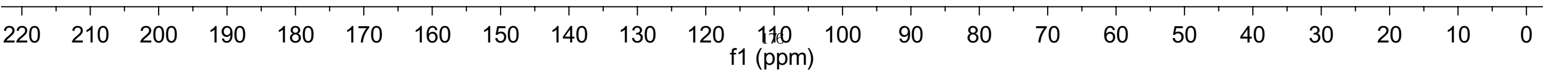




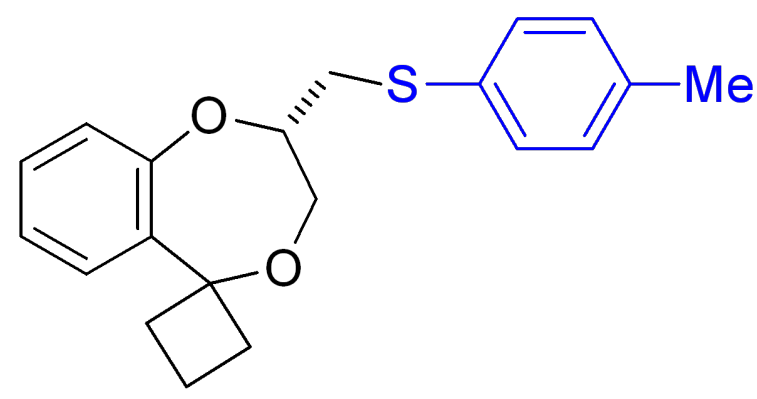

14

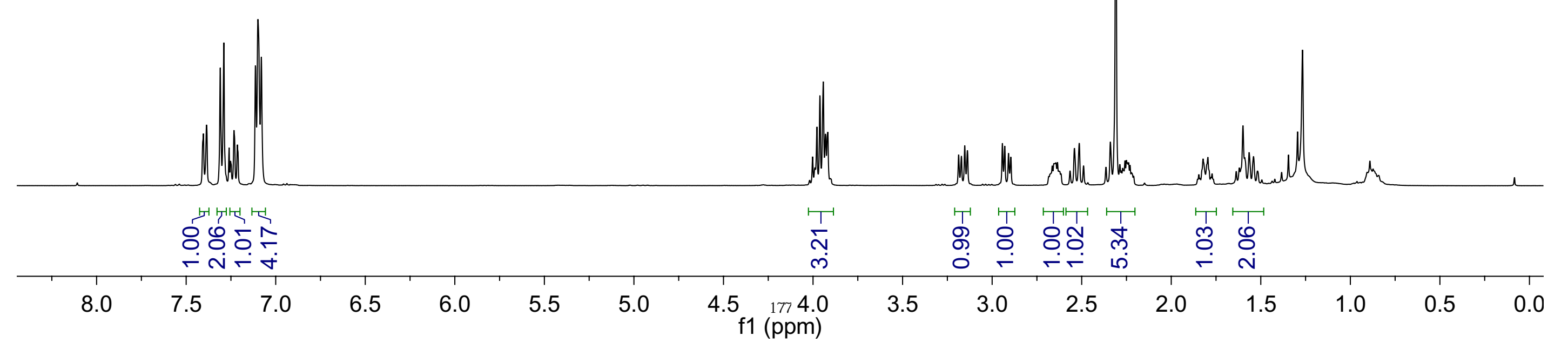




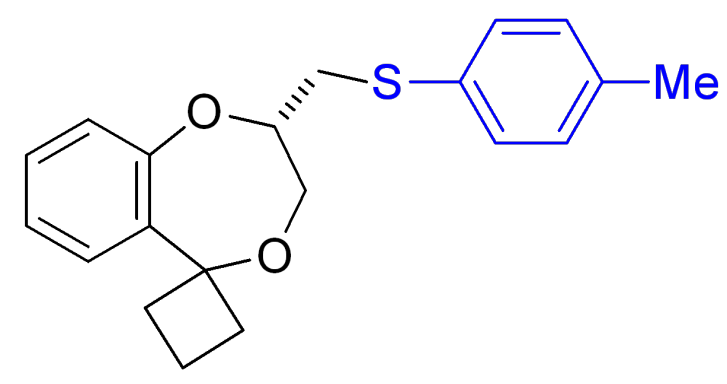

14

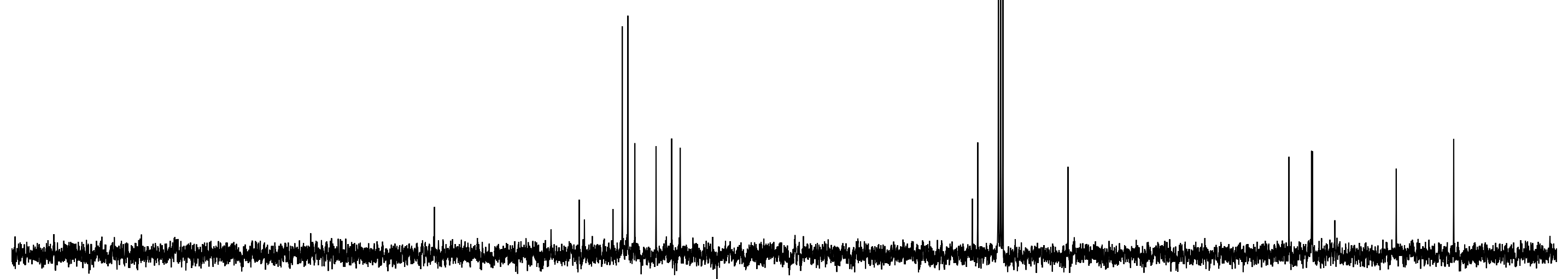

\begin{tabular}{|c|c|c|c|c|c|c|c|c|c|c|c|c|c|c|c|c|c|c|c|}
\hline 210 & 200 & 190 & 180 & 170 & 160 & 150 & 140 & 130 & 120 & 1108100 & 90 & 80 & 70 & 60 & 50 & 40 & 30 & 20 & 10 \\
\hline
\end{tabular}




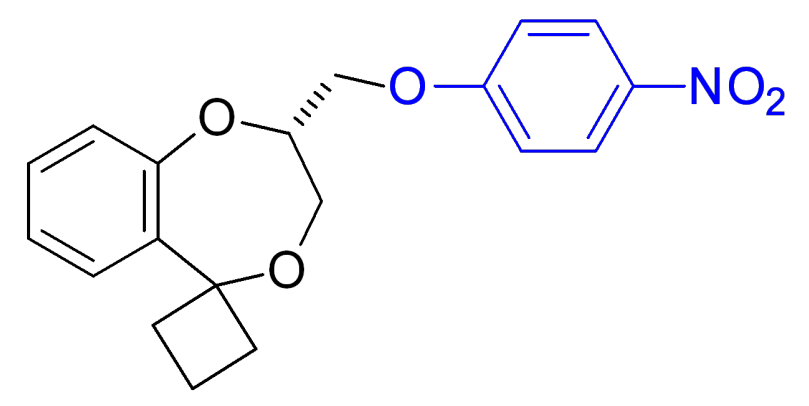

15

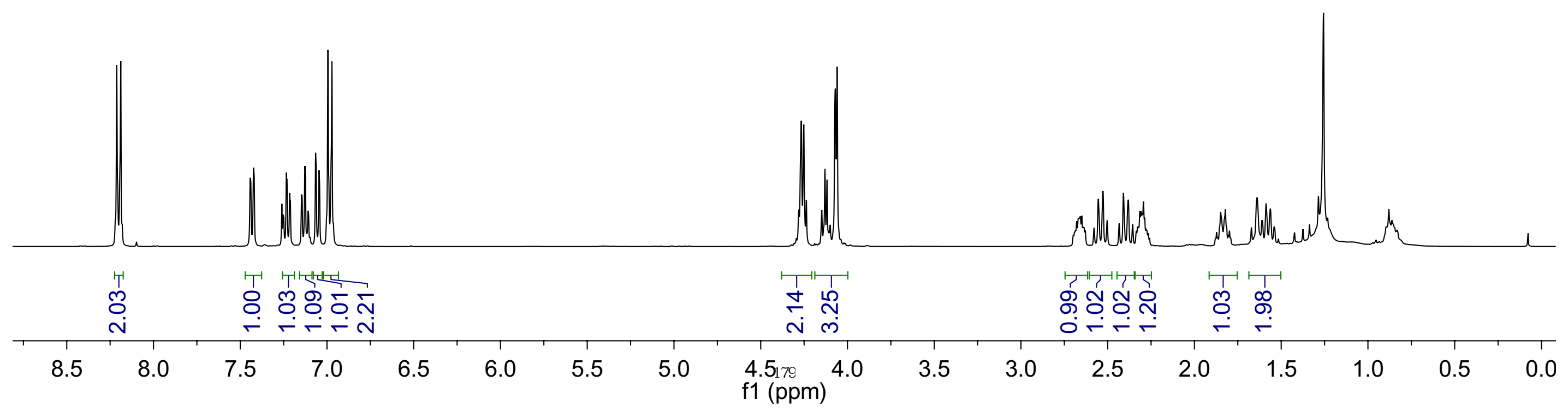




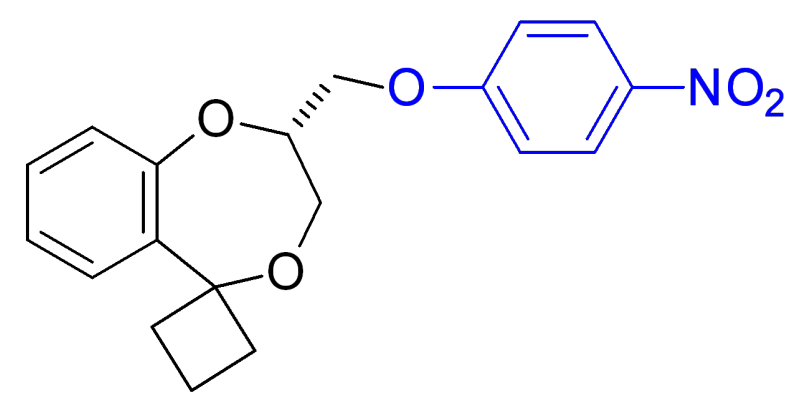

15

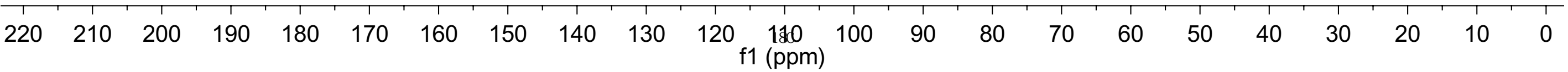




\section{Copies of HPLC profiles}

$\mathrm{mAU}$

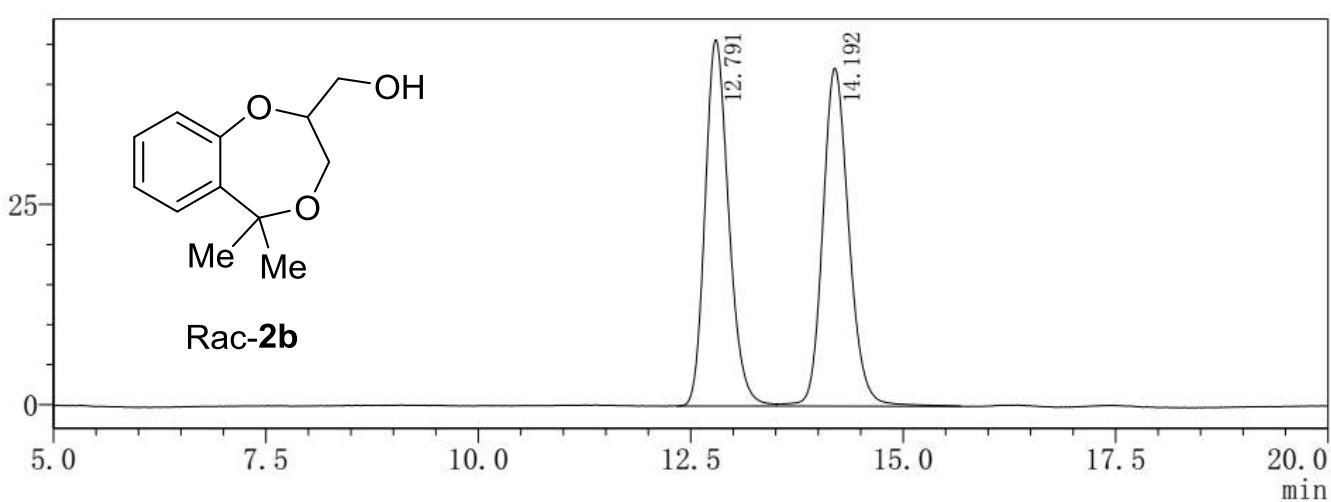

PDA Ch2 210nm 4nm

\begin{tabular}{|c|c|c|c|c|c|}
\hline Peak & Ret Time & Width & Area & Height & Area \% \\
\hline 1 & 12.791 & 0.509 & 884814 & 45782 & 49.463 \\
\hline 2 & 14.192 & 0.556 & 904044 & 42242 & 50.537 \\
\hline
\end{tabular}

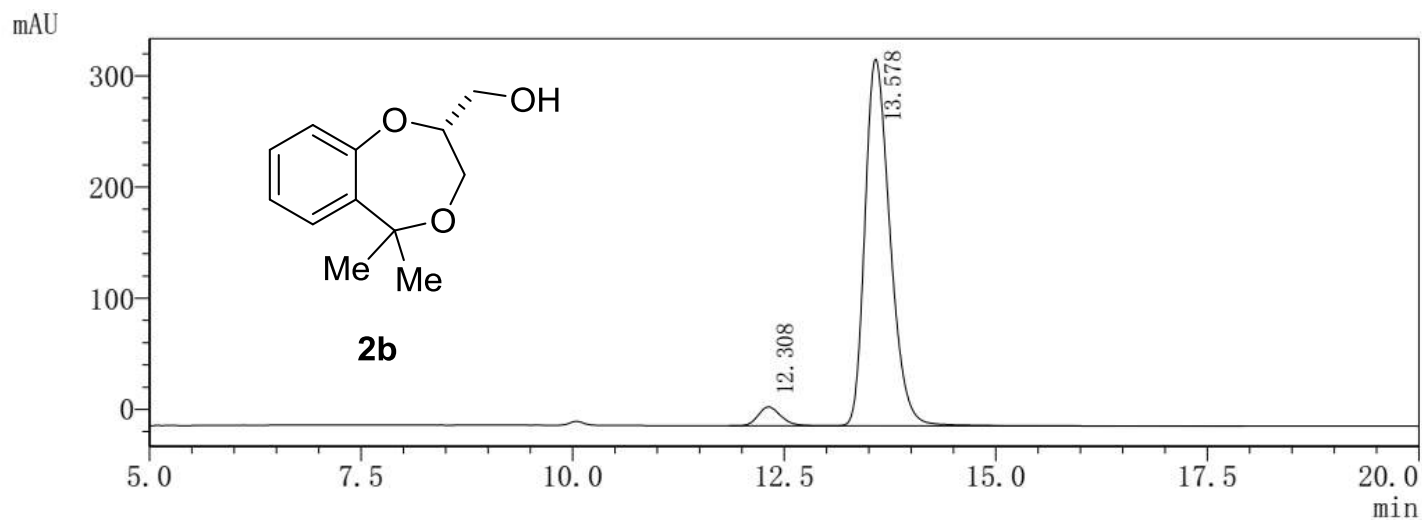

PDA Ch2 210nm 4nm

\begin{tabular}{|c|c|c|c|c|c|}
\hline Peak & Ret Time & Width & Area & Height & Area \% \\
\hline 1 & 12.308 & 0.497 & 322692 & 17041 & 4.533 \\
\hline 2 & 13.578 & 0.543 & 6795668 & 329905 & 95.467 \\
\hline
\end{tabular}




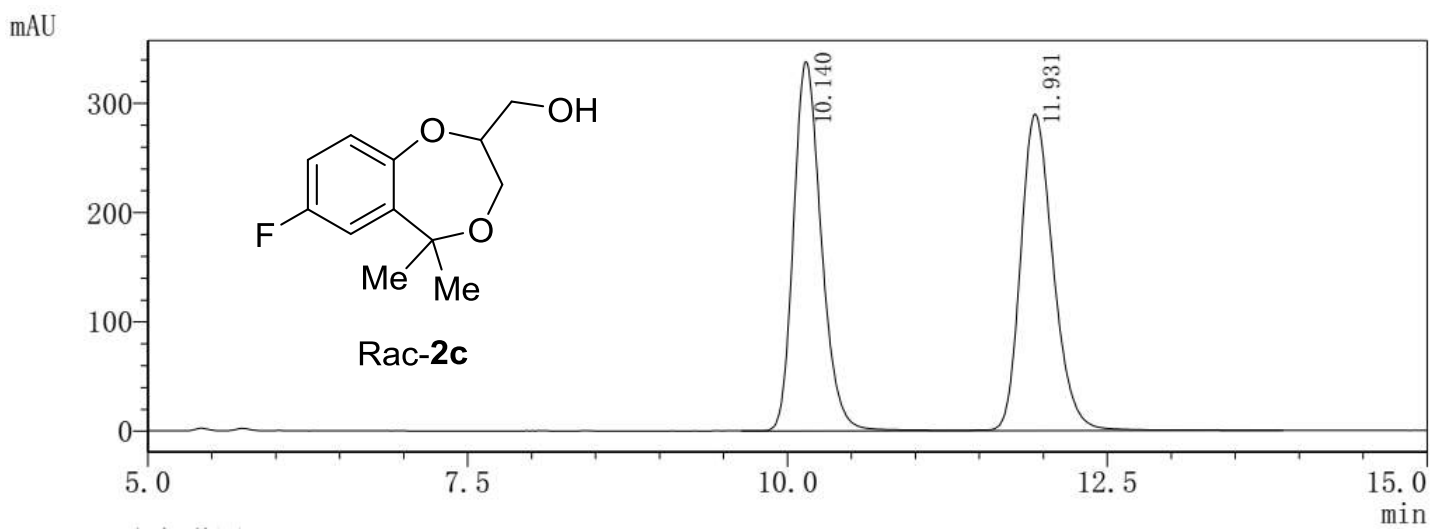

PDA Ch2 210nm 4nm

\begin{tabular}{|c|c|c|c|c|c|}
\hline Peak & Ret Time & Width & Area & Height & Area \% \\
\hline 1 & 10.140 & 0.400 & 5105804 & 338266 & 49.861 \\
\hline 2 & 11.931 & 0.467 & 5134337 & 289785 & 50.139 \\
\hline
\end{tabular}

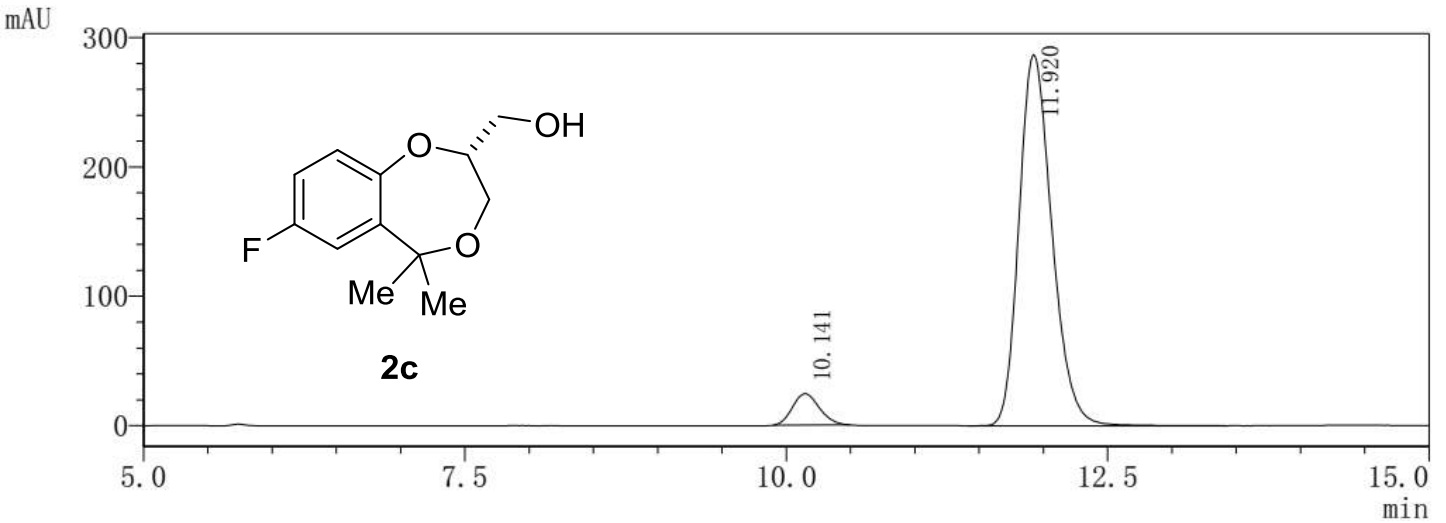

PDA Ch2 210nm 4nm

\begin{tabular}{|c|c|c|c|c|c|}
\hline Peak & Ret Time & Width & Area & Height & Area \% \\
\hline 1 & 10.141 & 0.399 & 355189 & 24303 & 6.491 \\
\hline 2 & 11.920 & 0.472 & 5116982 & 287219 & 93.509 \\
\hline
\end{tabular}




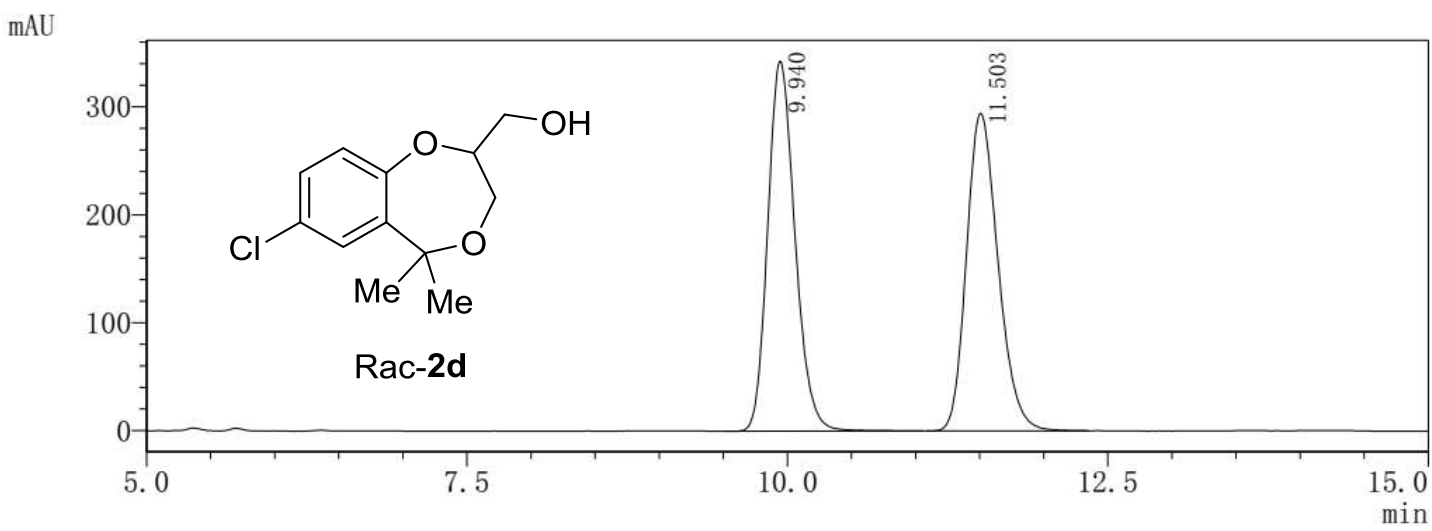

PDA Ch2 210nm 4nm

\begin{tabular}{|c|c|c|c|c|c|}
\hline Peak & Ret Time & Width & Area & Height & Area \% \\
\hline 1 & 9.940 & 0.394 & 5065595 & 342871 & 50.079 \\
\hline 2 & 11.503 & 0.457 & 5049625 & 294500 & 49.921 \\
\hline
\end{tabular}

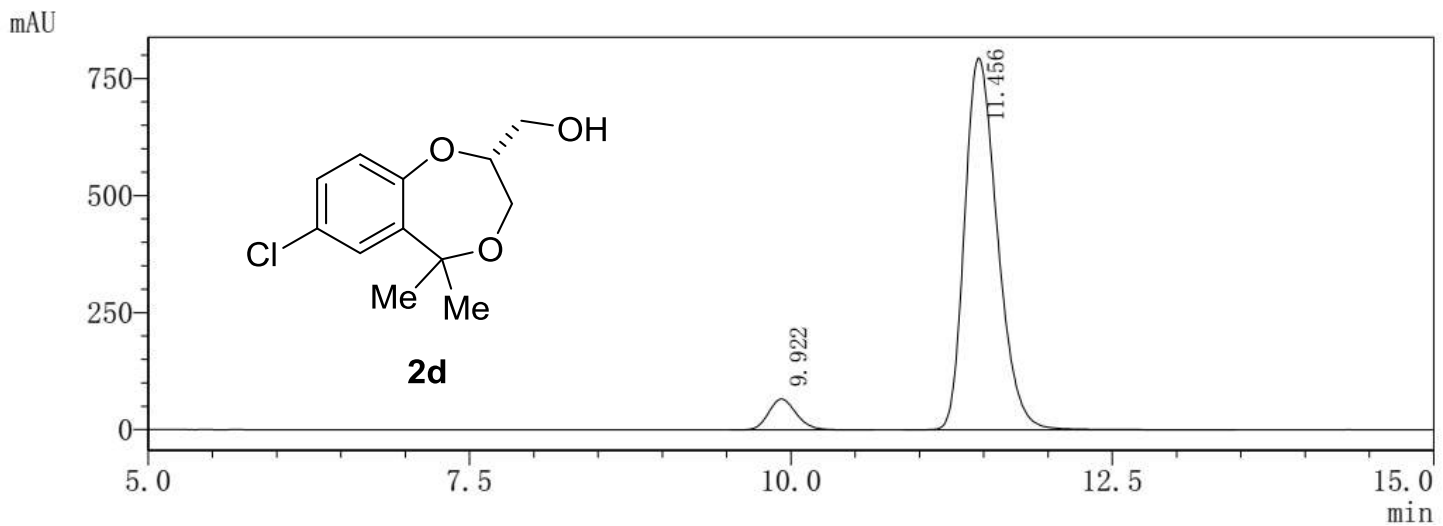

PDA Ch2 210nm 4nm

\begin{tabular}{|c|c|c|c|c|c|}
\hline Peak & Ret Time & Width & Area & Height & Area \% \\
\hline 1 & 9.922 & 0.401 & 985421 & 65913 & 6.525 \\
\hline 2 & 11.456 & 0.471 & 14116296 & 794137 & 93.475 \\
\hline
\end{tabular}




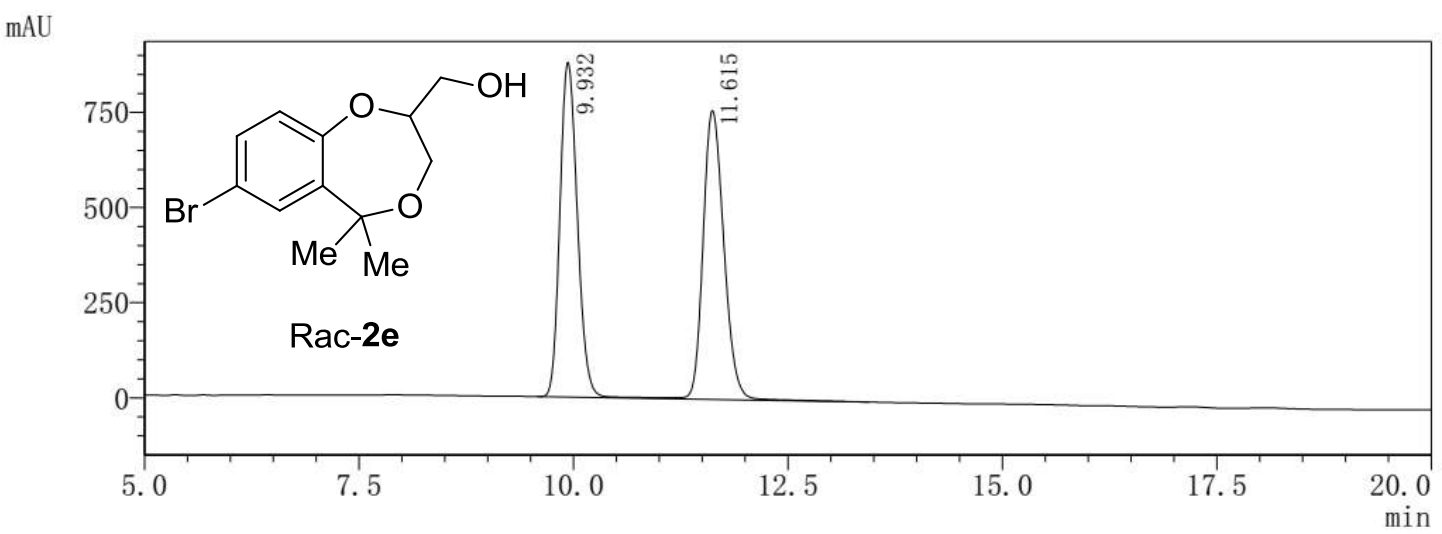

PDA Ch2 210nm 4nm

\begin{tabular}{|c|c|c|c|c|c|}
\hline Peak & Ret Time & Width & Area & Height & Area \% \\
\hline 1 & 9.932 & 0.378 & 12474390 & 880037 & 49.312 \\
\hline 2 & 11.615 & 0.445 & 12822578 & 759636 & 50.688 \\
\hline
\end{tabular}

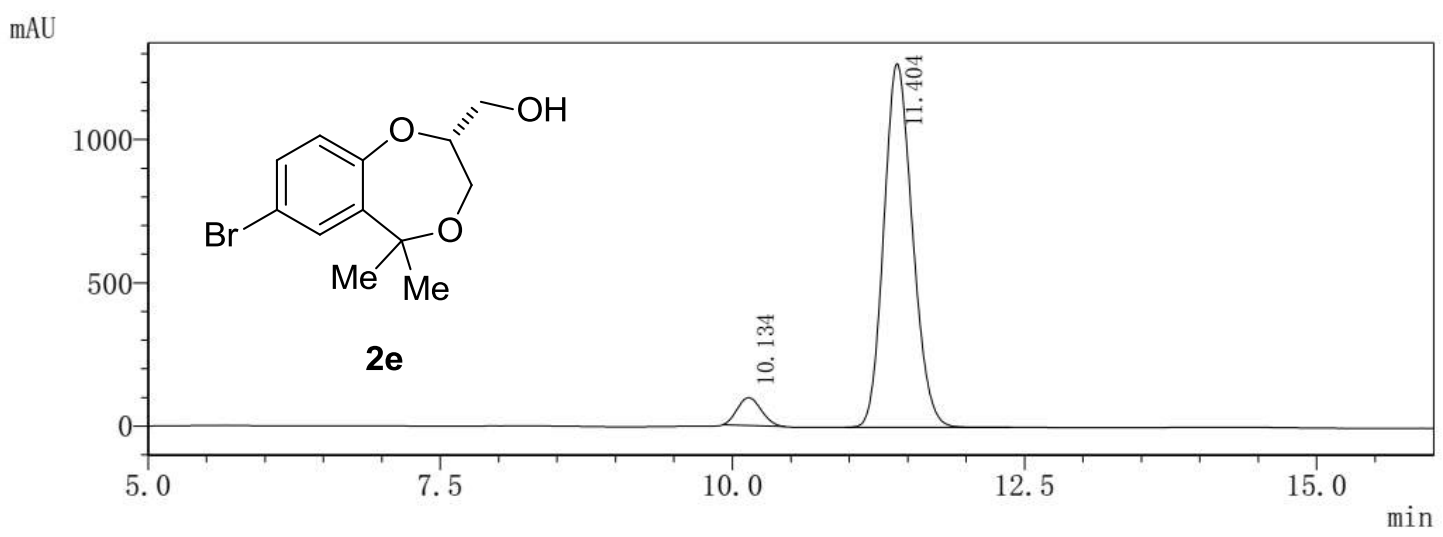

PDA Ch2 210nm 4nm

\begin{tabular}{|c|c|c|c|c|c|}
\hline Peak & Ret Time & Width & Area & Height & Area \% \\
\hline 1 & 10.134 & 0.394 & 1381716 & 96968 & 6.003 \\
\hline 2 & 11.404 & 0.461 & 21637031 & 1269621 & 93.997 \\
\hline
\end{tabular}




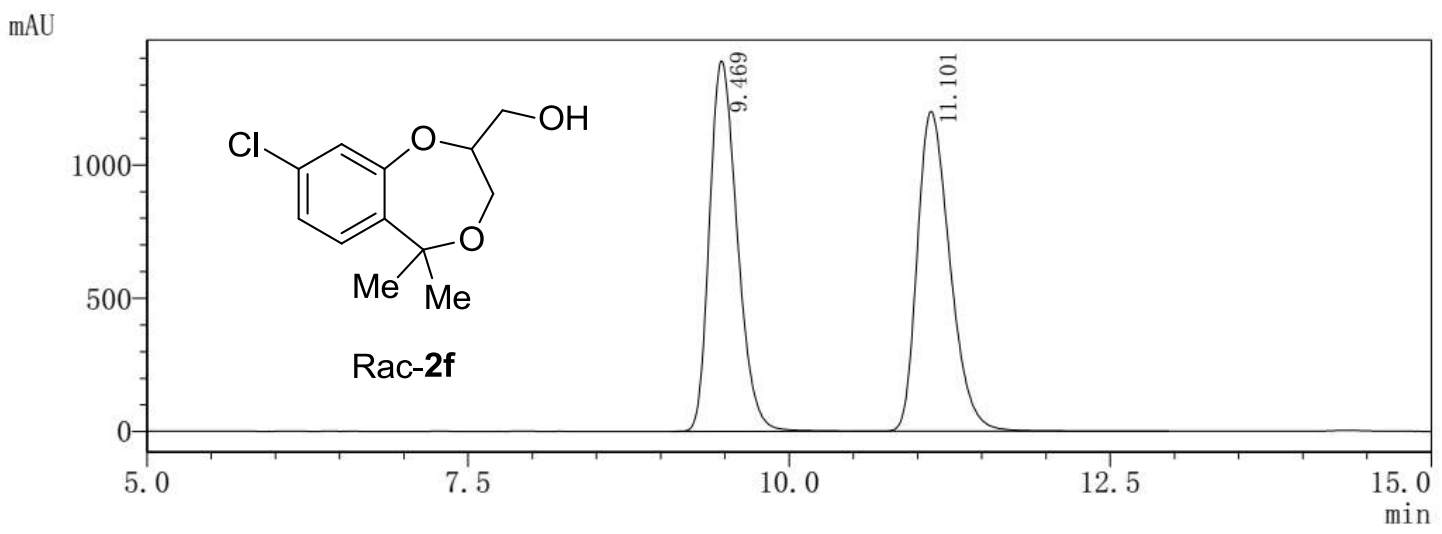

PDA Ch2 210nm 4nm

\begin{tabular}{|c|c|c|c|c|c|}
\hline Peak & Ret Time & Width & Area & Height & Area \% \\
\hline 1 & 9.469 & 0.403 & 20897954 & 1390129 & 49.564 \\
\hline 2 & 11.101 & 0.471 & 21265445 & 1200630 & 50.436 \\
\hline
\end{tabular}

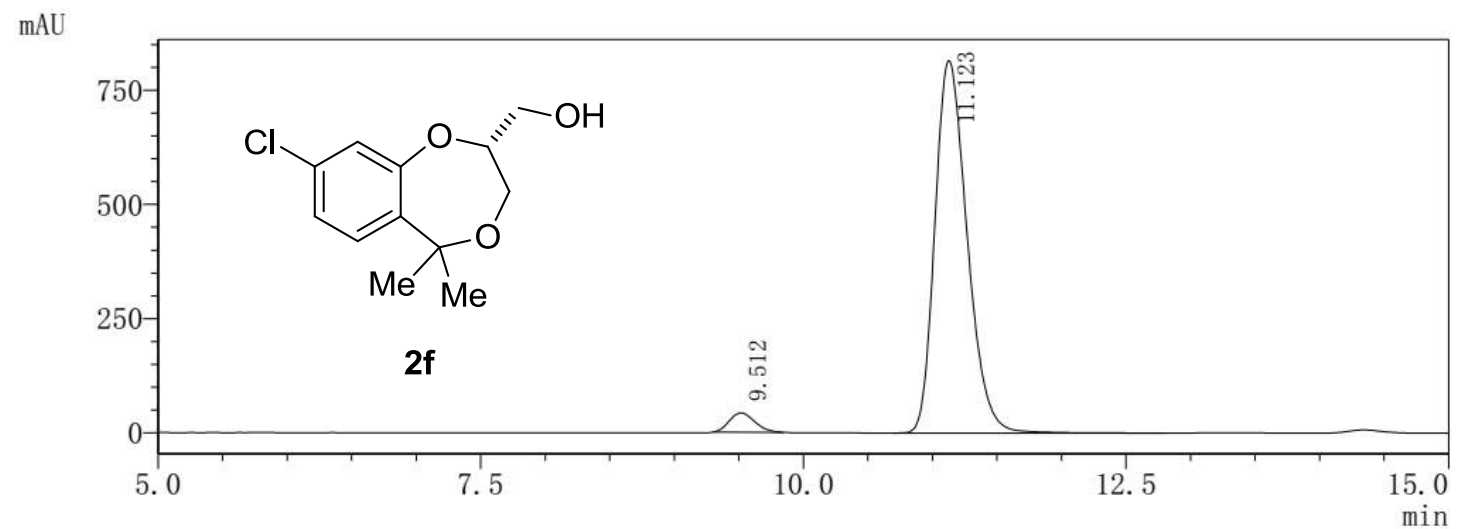

PDA Ch2 210nm 4nm

\begin{tabular}{|c|c|c|c|c|c|}
\hline Peak & Ret Time & Width & Area & Height & Area \% \\
\hline 1 & 9.512 & 0.376 & 580576 & 42429 & 3.937 \\
\hline 2 & 11.123 & 0.462 & 14167391 & 815531 & 96.063 \\
\hline
\end{tabular}




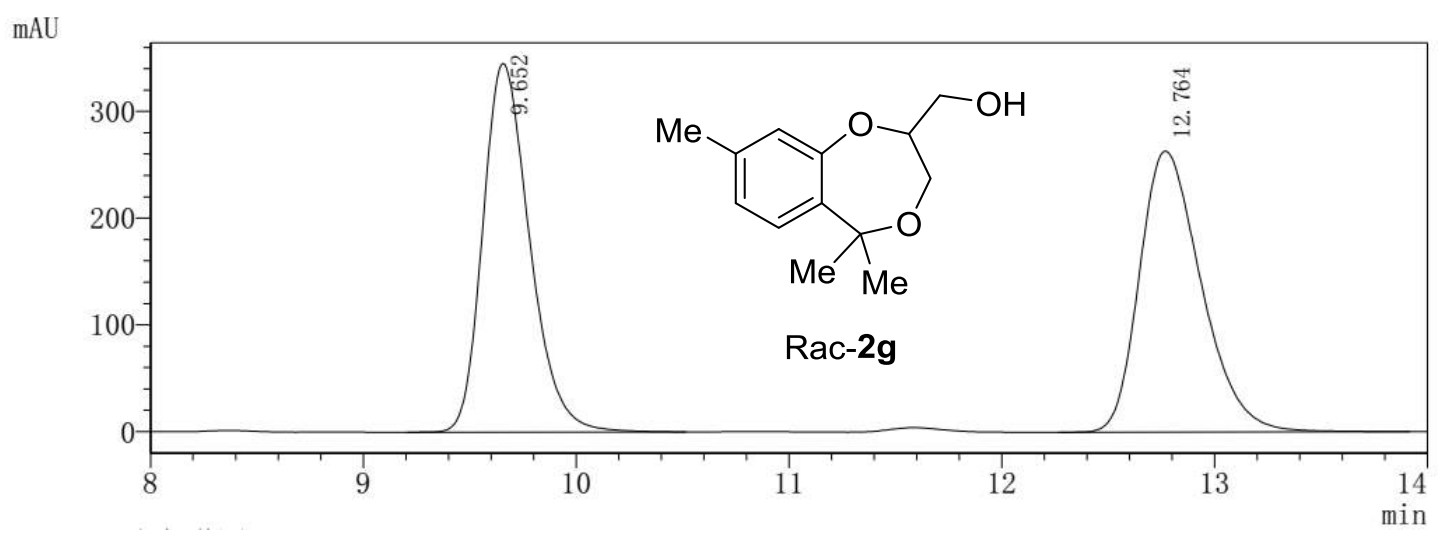

PDA Ch2 210nm 4nm

\begin{tabular}{|c|c|c|c|c|c|}
\hline Peak & Ret Time & Width & Area & Height & Area \% \\
\hline 1 & 9.652 & 0.407 & 5295425 & 345410 & 49.858 \\
\hline 2 & 12.764 & 0.535 & 5325576 & 263456 & 50.142 \\
\hline
\end{tabular}

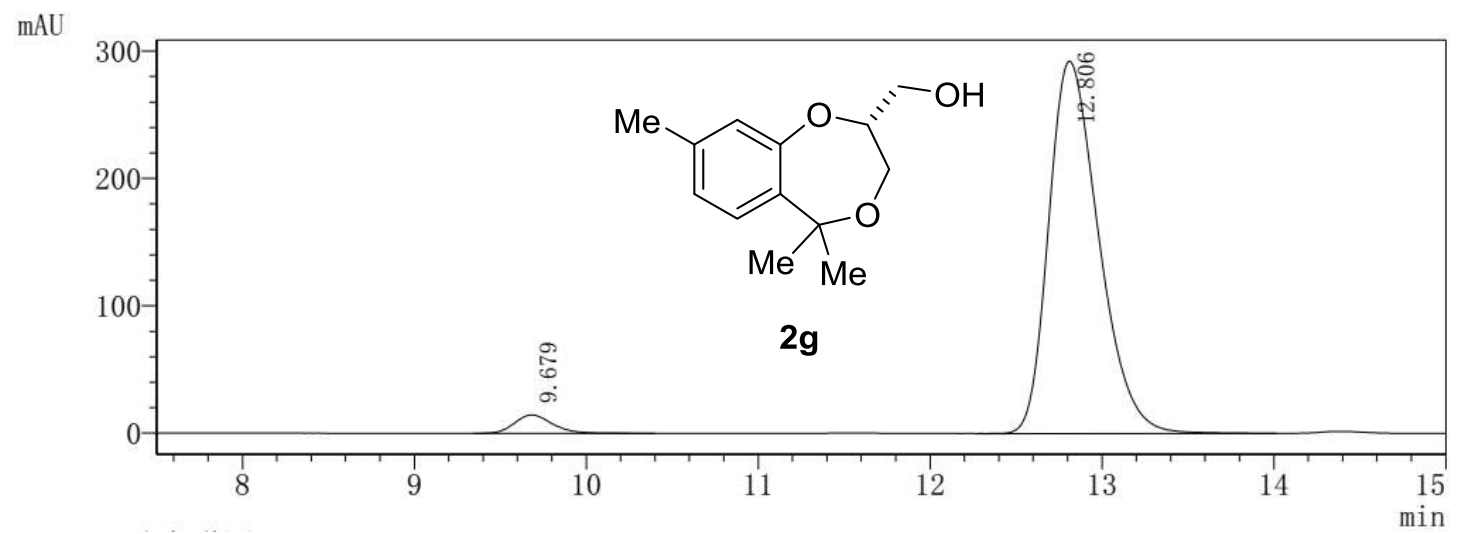

PDA Ch2 210nm 4nm

\begin{tabular}{|c|c|c|c|c|c|}
\hline Peak & Ret Time & Width & Area & Height & Area \% \\
\hline 1 & 9.679 & 0.405 & 220572 & 14443 & 3.601 \\
\hline 2 & 12.806 & 0.536 & 5905368 & 292553 & 96.399 \\
\hline
\end{tabular}




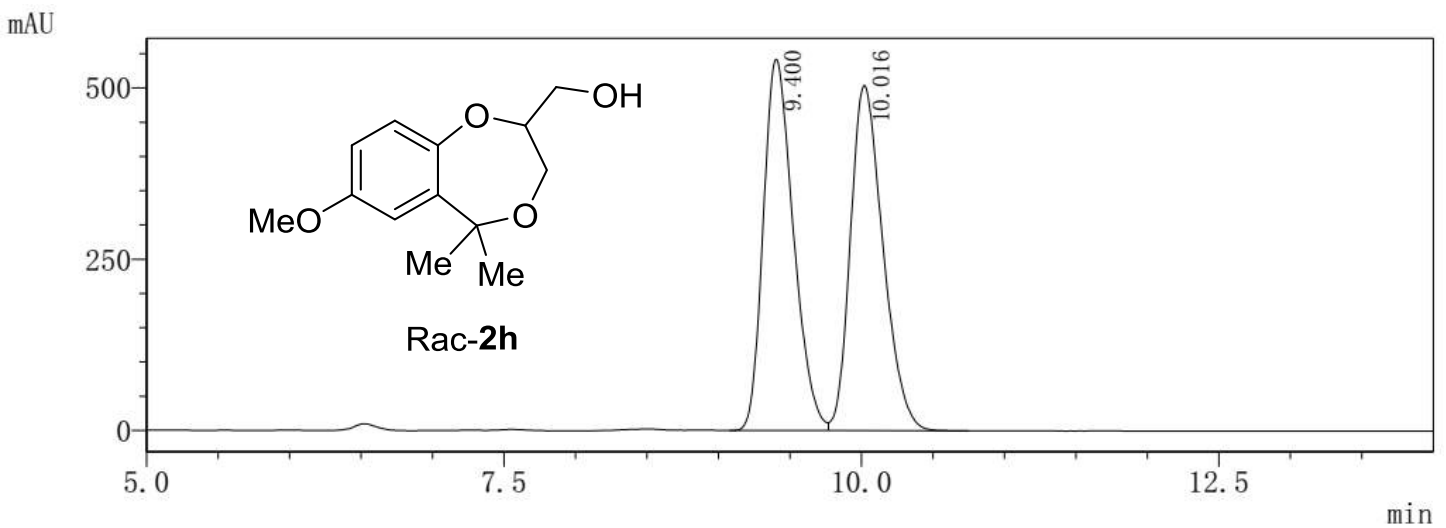

PDA Ch2 210nm 4nm

\begin{tabular}{|c|c|c|c|c|c|}
\hline Peak & Ret Time & Width & Area & Height & Area \% \\
\hline 1 & 9.400 & 0.398 & 7947952 & 542283 & 49.885 \\
\hline 2 & 10.016 & 0.428 & 7984521 & 503908 & 50.115 \\
\hline
\end{tabular}

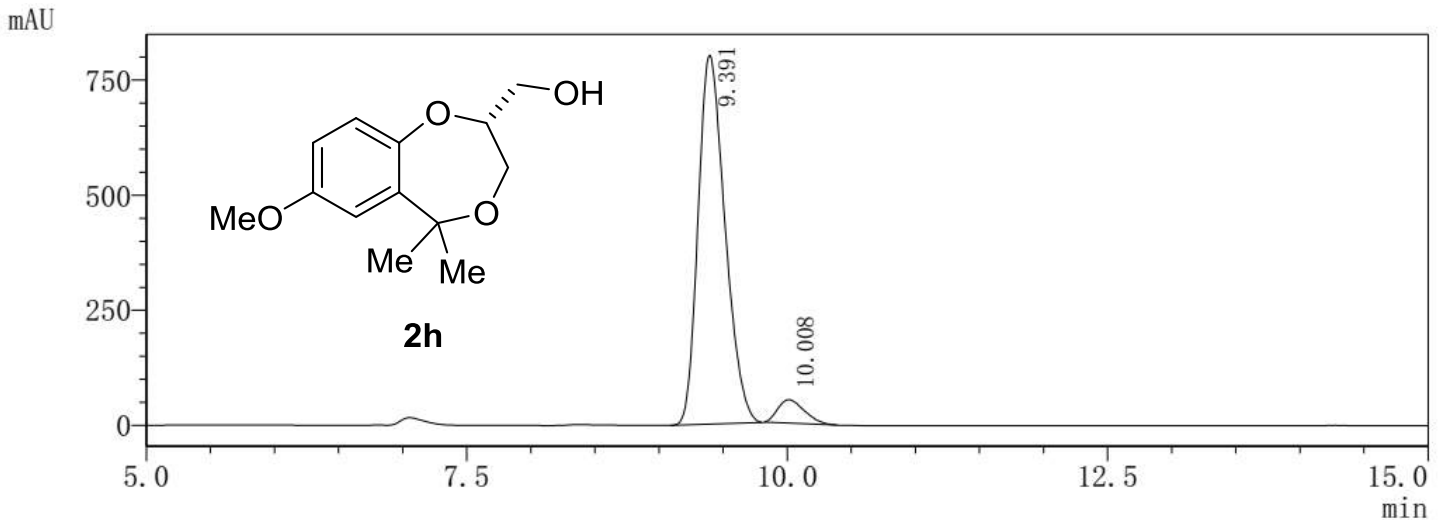

PDA Ch2 210nm 4nm

\begin{tabular}{|c|c|c|c|c|c|}
\hline Peak & Ret Time & Width & Area & Height & Area \% \\
\hline 1 & 9.391 & 0.399 & 11692737 & 801197 & 94.093 \\
\hline 2 & 10.008 & 0.400 & 734091 & 50882 & 5.907 \\
\hline
\end{tabular}




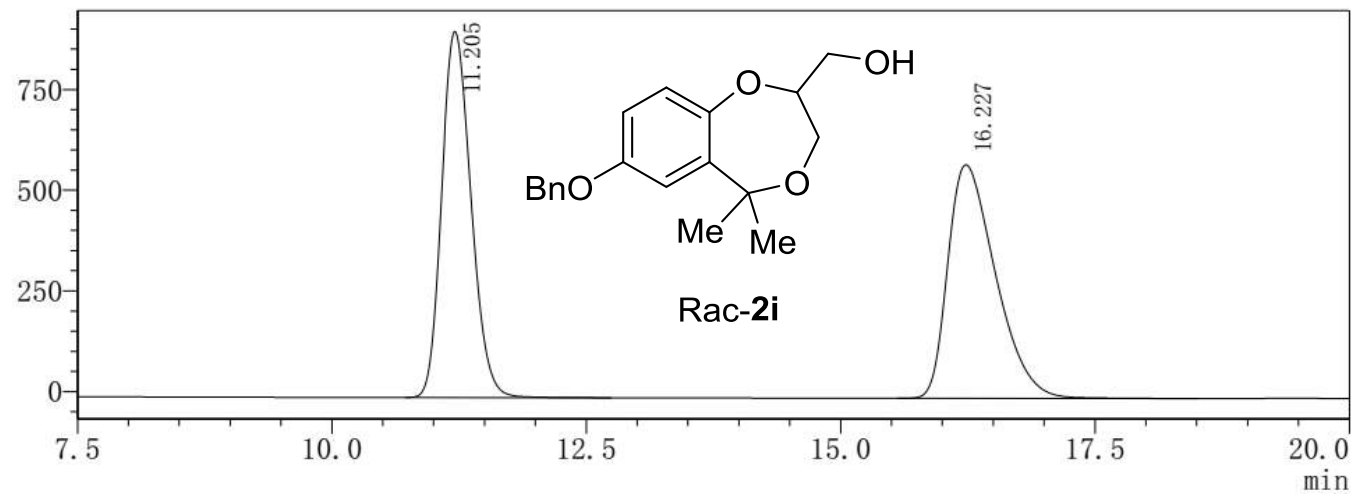

PDA Ch2 210nm 4nm

\begin{tabular}{|c|c|c|c|c|c|}
\hline Peak & Ret Time & Width & Area & Height & Area \% \\
\hline 1 & 11.205 & 0.536 & 18344828 & 909390 & 49.239 \\
\hline 2 & 16.227 & 0.863 & 18911678 & 579966 & 50.761 \\
\hline
\end{tabular}

$\mathrm{mAU}$

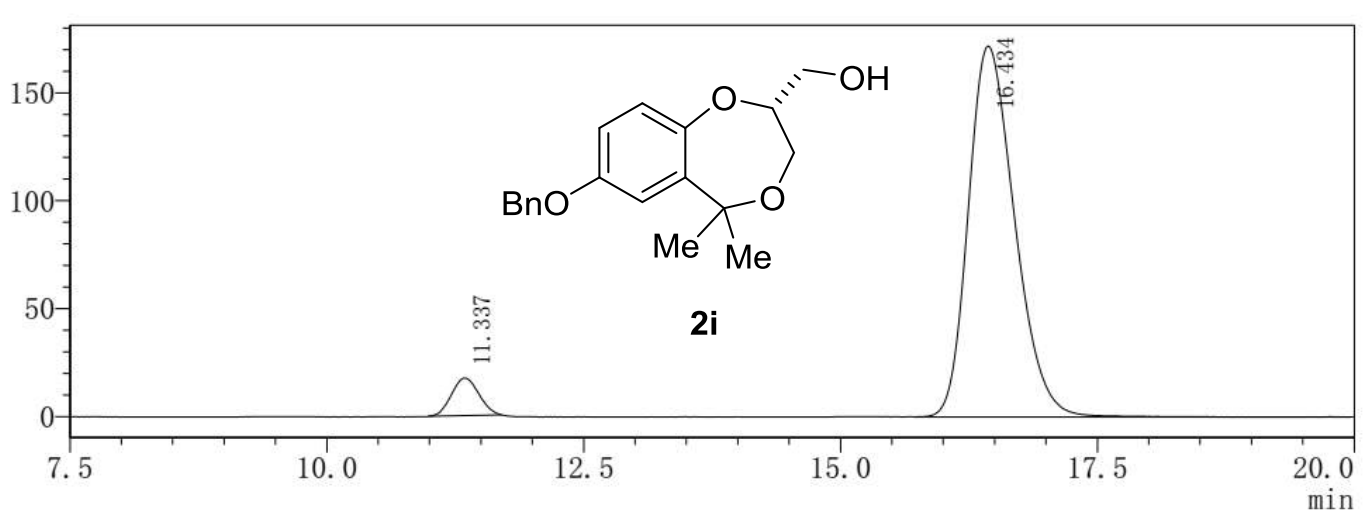

PDA Ch2 210nm 4nm

\begin{tabular}{|c|c|c|c|c|c|}
\hline Peak & Ret Time & Width & Area & Height & Area \% \\
\hline 1 & 11.337 & 0.507 & 322065 & 17394 & 5.656 \\
\hline 2 & 16.434 & 0.825 & 5372184 & 171866 & 94.344 \\
\hline
\end{tabular}




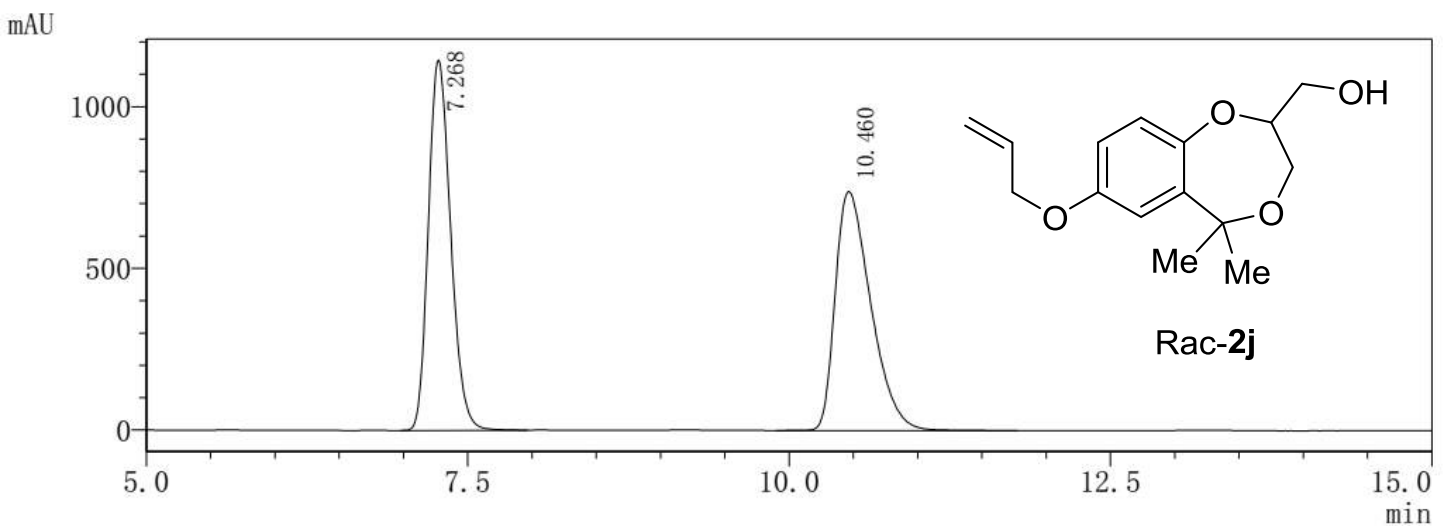

PDA Ch2 210nm 4nm

\begin{tabular}{|c|c|c|c|c|c|}
\hline Peak & Ret Time & Width & Area & Height & Area \% \\
\hline 1 & 7.268 & 0.326 & 13797486 & 1145766 & 49.239 \\
\hline 2 & 10.460 & 0.513 & 14223810 & 739721 & 50.761 \\
\hline
\end{tabular}

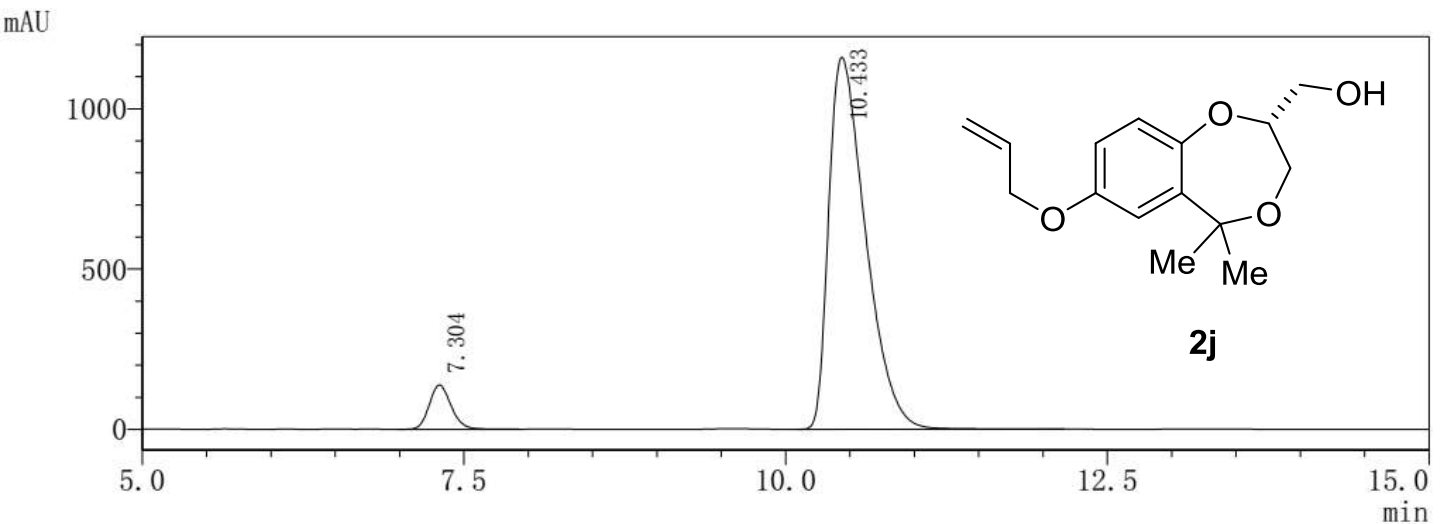

PDA Ch2 210nm 4nm

\begin{tabular}{|c|c|c|c|c|c|}
\hline Peak & Ret Time & Width & Area & Height & Area \% \\
\hline 1 & 7.304 & 0.319 & 1634057 & 138347 & 6.604 \\
\hline 2 & 10.433 & 0.530 & 23107631 & 1160067 & 93.396 \\
\hline
\end{tabular}




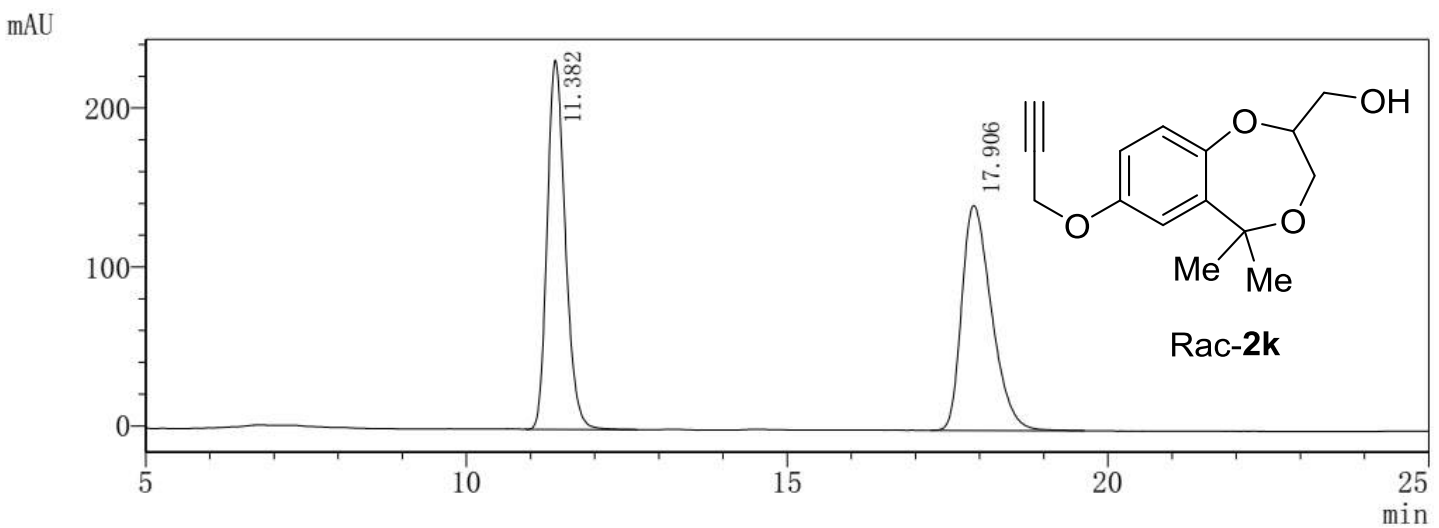

PDA Ch2 210nm 4nm

\begin{tabular}{|c|c|c|c|c|c|}
\hline Peak & Ret Time & Width & Area & Height & Area \% \\
\hline 1 & 11.382 & 0.519 & 4554182 & 232327 & 49.875 \\
\hline 2 & 17.906 & 0.851 & 4577099 & 141598 & 50.125 \\
\hline
\end{tabular}

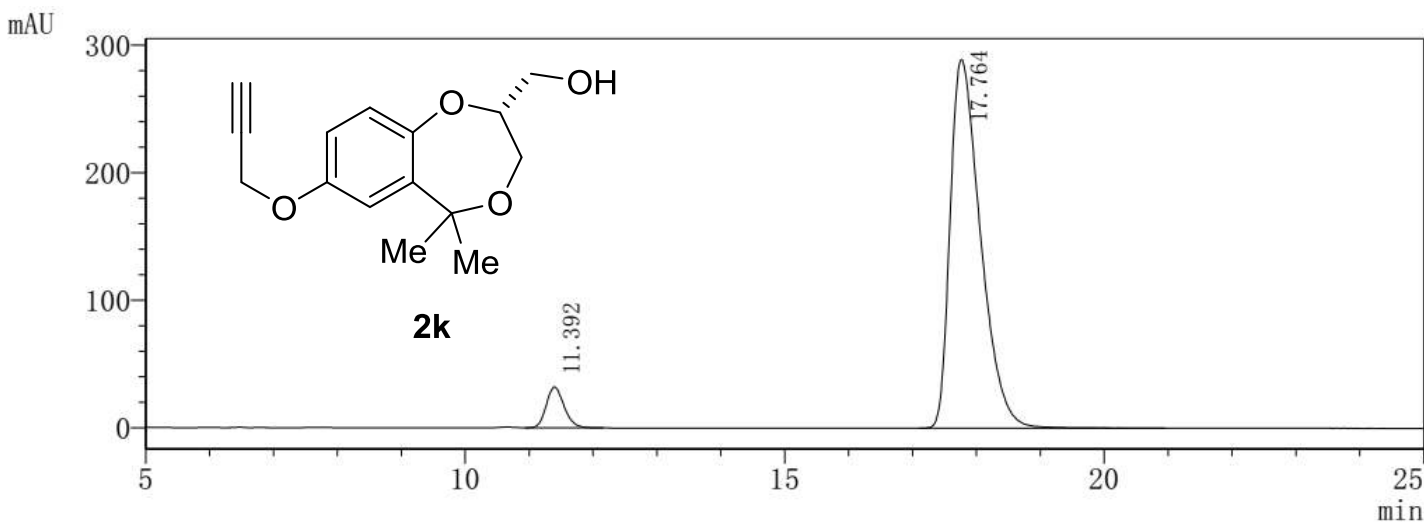

PDA Ch2 210nm 4nm

\begin{tabular}{|c|c|c|c|c|c|}
\hline Peak & Ret Time & Width & Area & Height & Area \% \\
\hline 1 & 11.392 & 0.516 & 620941 & 31899 & 6.094 \\
\hline 2 & 17.764 & 0.869 & 9567651 & 289286 & 93.906 \\
\hline
\end{tabular}




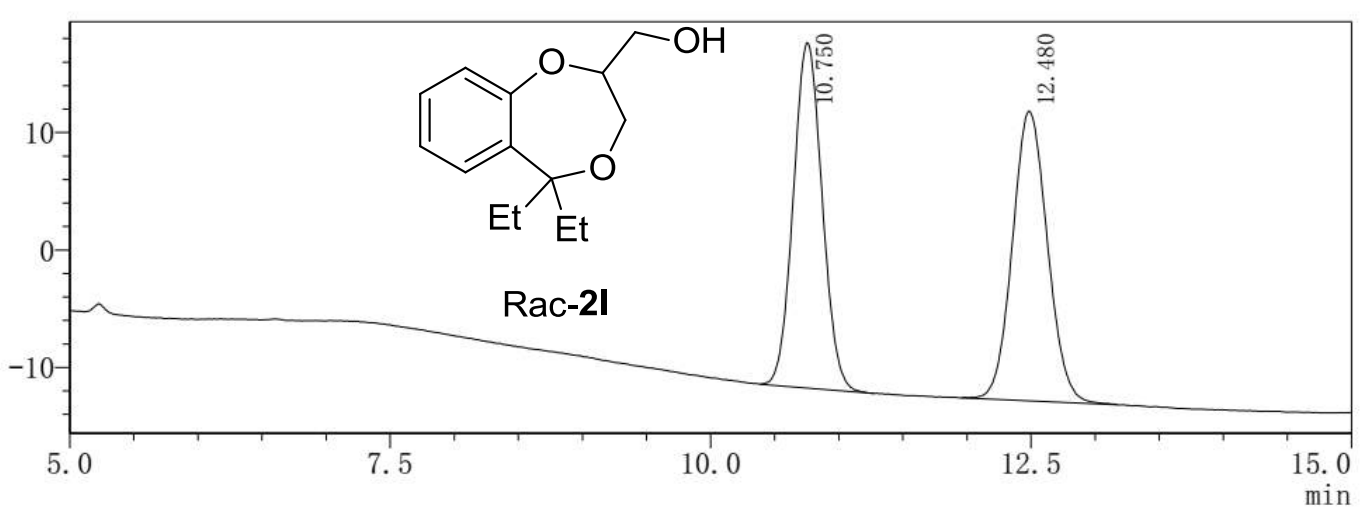

PDA Ch2 210nm 4nm

\begin{tabular}{|c|c|c|c|c|c|}
\hline Peak & Ret Time & Width & Area & Height & Area \% \\
\hline 1 & 10.750 & 0.424 & 461558 & 29425 & 49.573 \\
\hline 2 & 12.480 & 0.507 & 469510 & 24655 & 50.427 \\
\hline
\end{tabular}

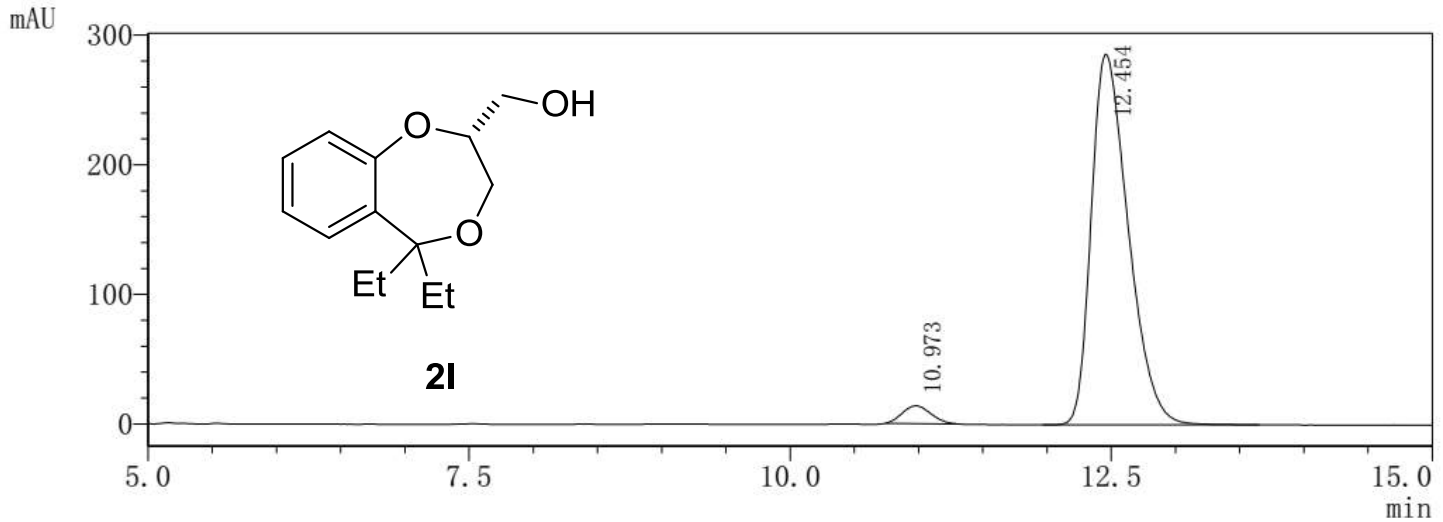

PDA Ch2 210nm 4nm

\begin{tabular}{|c|c|c|c|c|c|}
\hline Peak & Ret Time & Width & Area & Height & Area \% \\
\hline 1 & 10.973 & 0.427 & 213070 & 13767 & 3.585 \\
\hline 2 & 12.454 & 0.535 & 5730741 & 286050 & 96.415 \\
\hline
\end{tabular}




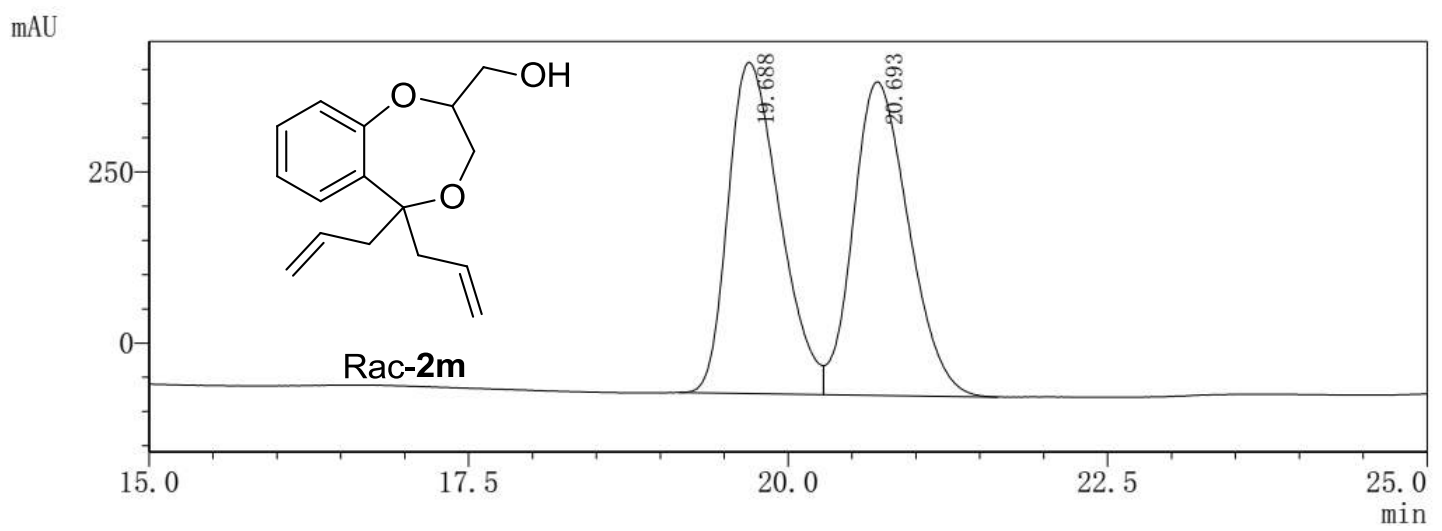

PDA Ch2 210nm 4nm

\begin{tabular}{|c|c|c|c|c|c|}
\hline Peak & Ret Time & Width & Area & Height & Area \% \\
\hline 1 & 19.688 & 0.754 & 13633861 & 484329 & 49.690 \\
\hline 2 & 20.693 & 0.802 & 13804095 & 458104 & 50.310 \\
\hline
\end{tabular}

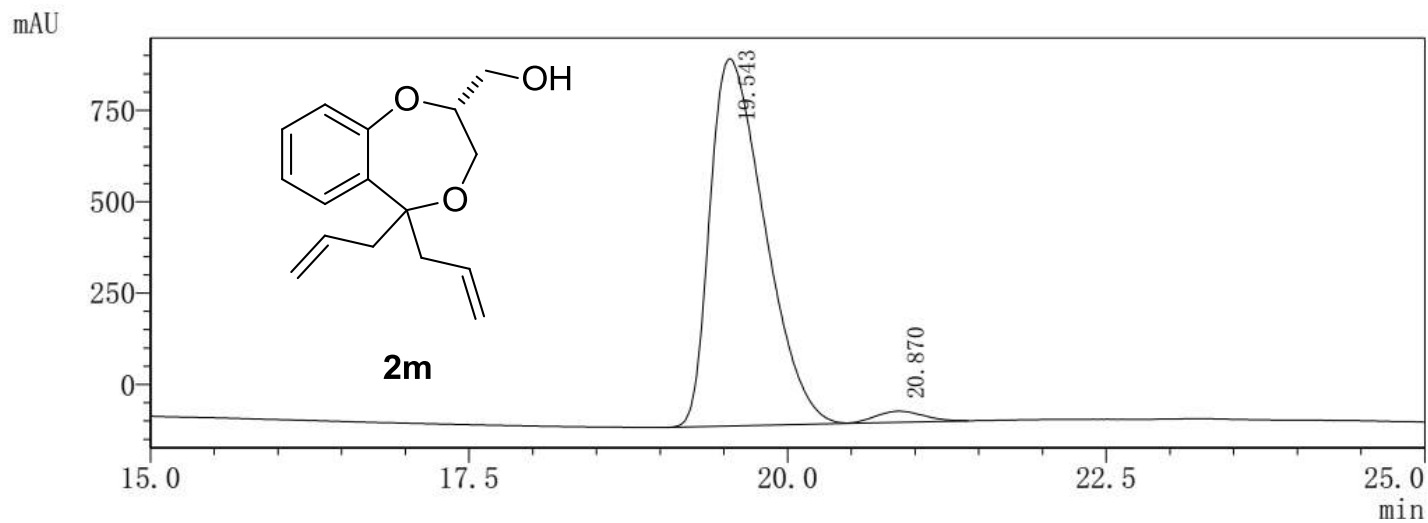

PDA Ch2 210nm 4nm

\begin{tabular}{|c|c|c|c|c|c|}
\hline Peak & Ret Time & Width & Area & Height & Area \% \\
\hline 1 & 19.543 & 0.804 & 30036286 & 1005394 & 97.430 \\
\hline 2 & 20.870 & 0.703 & 792239 & 30499 & 2.570 \\
\hline
\end{tabular}




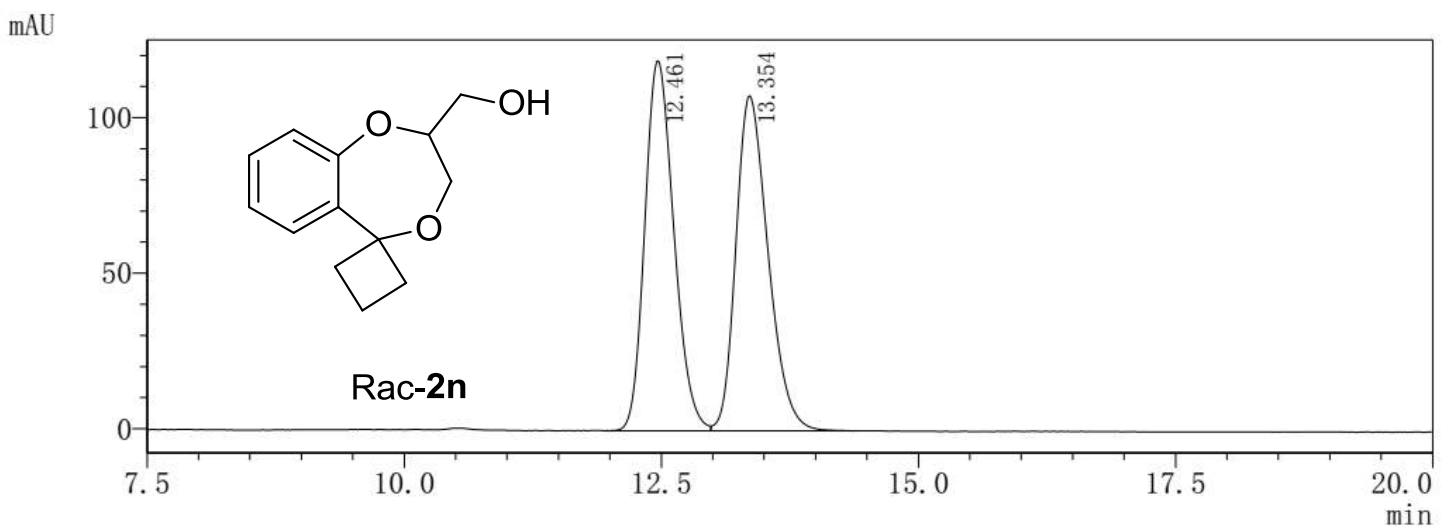

PDA Ch2 210nm 4nm

\begin{tabular}{|c|c|c|c|c|c|}
\hline Peak & Ret Time & Width & Area & Height & Area \% \\
\hline 1 & 12.461 & 0.525 & 2338538 & 119023 & 49.721 \\
\hline 2 & 13.354 & 0.582 & 2364778 & 107694 & 50.279 \\
\hline
\end{tabular}

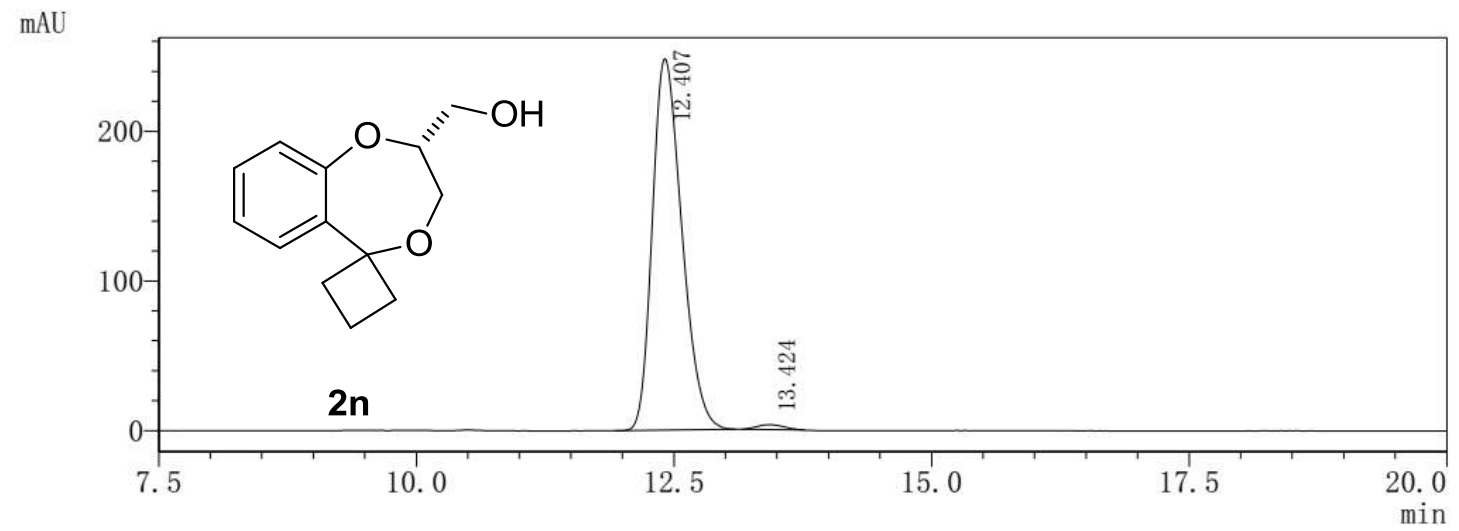

PDA Ch2 210nm 4nm

\begin{tabular}{|c|c|c|c|c|c|}
\hline Peak & Ret Time & Width & Area & Height & Area \% \\
\hline 1 & 12.407 & 0.533 & 4946020 & 248298 & 98.768 \\
\hline 2 & 13.424 & 0.521 & 61682 & 3332 & 1.232 \\
\hline
\end{tabular}




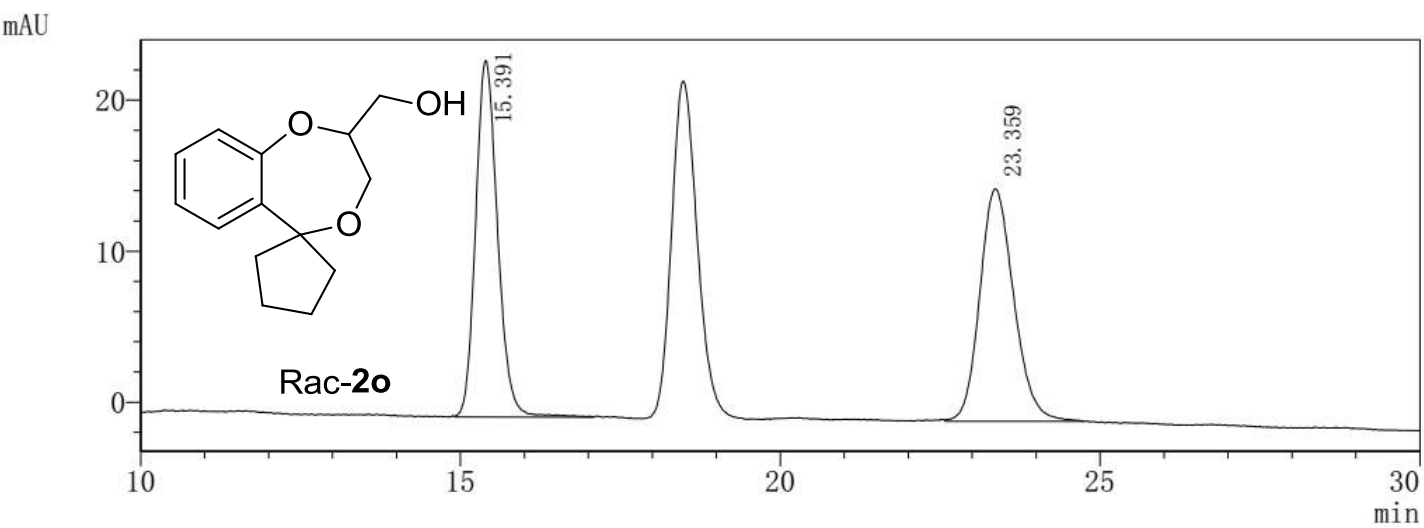

PDA Ch2 210nm 4nm

\begin{tabular}{|c|c|c|c|c|c|}
\hline Peak & Ret Time & Width & Area & Height & Area \% \\
\hline 1 & 15.391 & 0.625 & 562572 & 23616 & 49.668 \\
\hline 2 & 23.359 & 0.965 & 570084 & 15398 & 50.332 \\
\hline
\end{tabular}

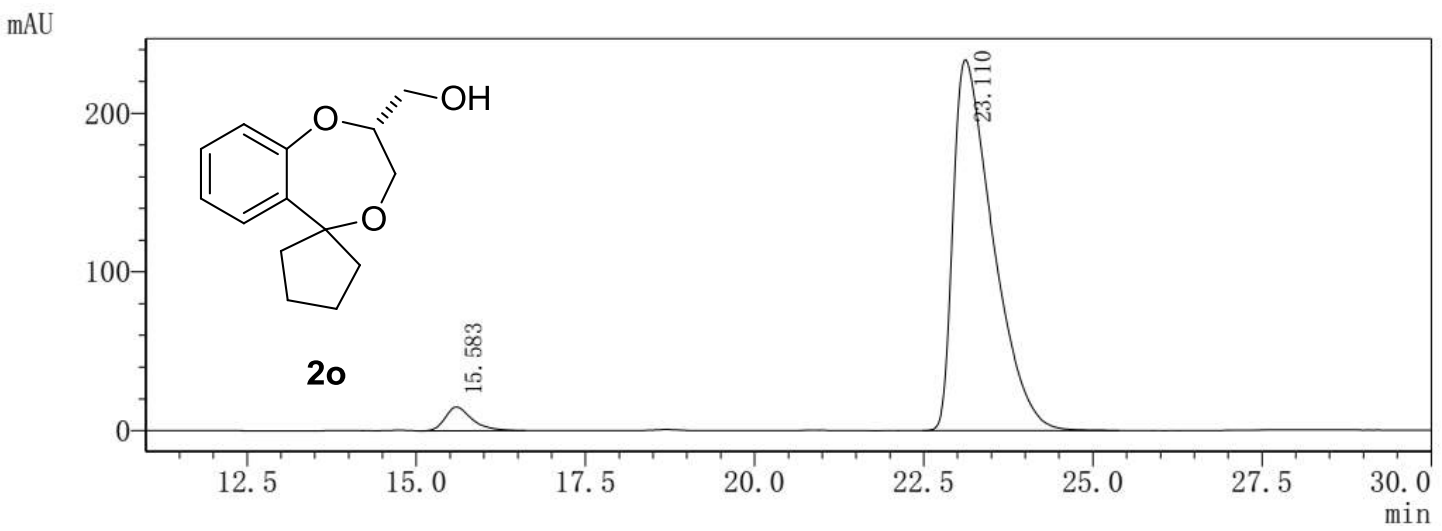

PDA Ch2 210nm 4nm

\begin{tabular}{|c|c|c|c|c|c|}
\hline Peak & Ret Time & Width & Area & Height & Area \% \\
\hline 1 & 15.583 & 0.701 & 412884 & 14951 & 4.146 \\
\hline 2 & 23.110 & 1.081 & 9544999 & 233905 & 95.854 \\
\hline
\end{tabular}




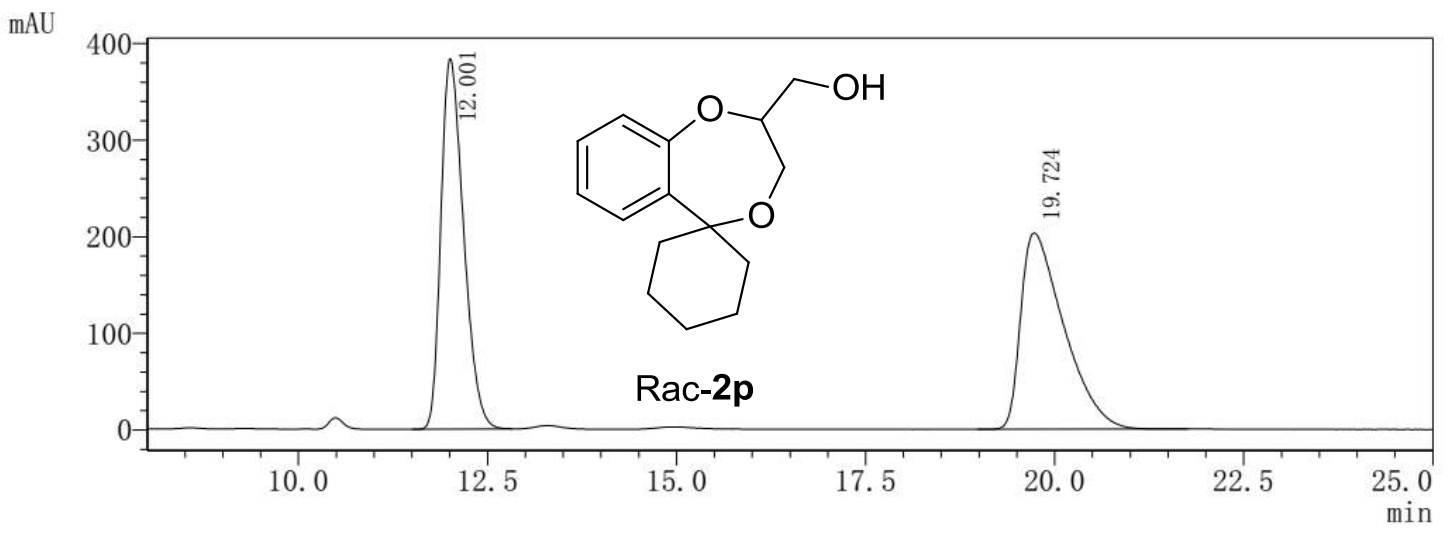

PDA Ch2 210nm 4nm

\begin{tabular}{|c|c|c|c|c|c|}
\hline Peak & Ret Time & Width & Area & Height & Area \% \\
\hline 1 & 12.001 & 0.559 & 8007820 & 383217 & 49.866 \\
\hline 2 & 19.724 & 1.055 & 8050710 & 203113 & 50.134 \\
\hline
\end{tabular}

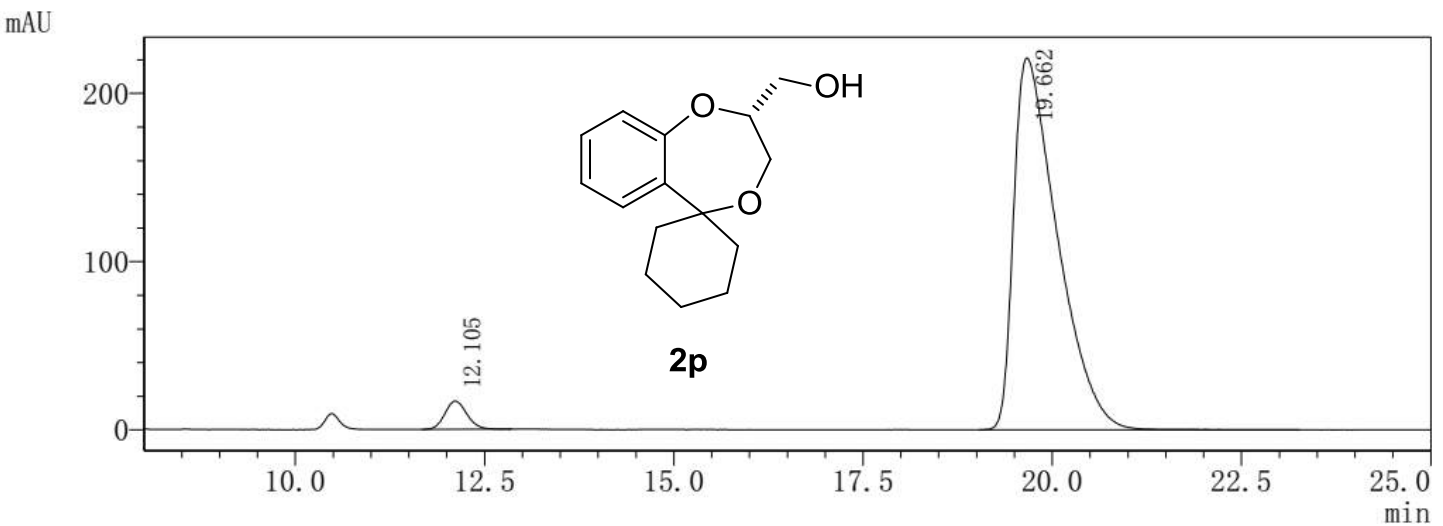

PDA Ch2 210nm 4nm

\begin{tabular}{|c|c|c|c|c|c|}
\hline Peak & Ret Time & Width & Area & Height & Area \% \\
\hline 1 & 12.105 & 0.541 & 342983 & 16863 & 3.727 \\
\hline 2 & 19.662 & 1.065 & 8859187 & 221003 & 96.273 \\
\hline
\end{tabular}




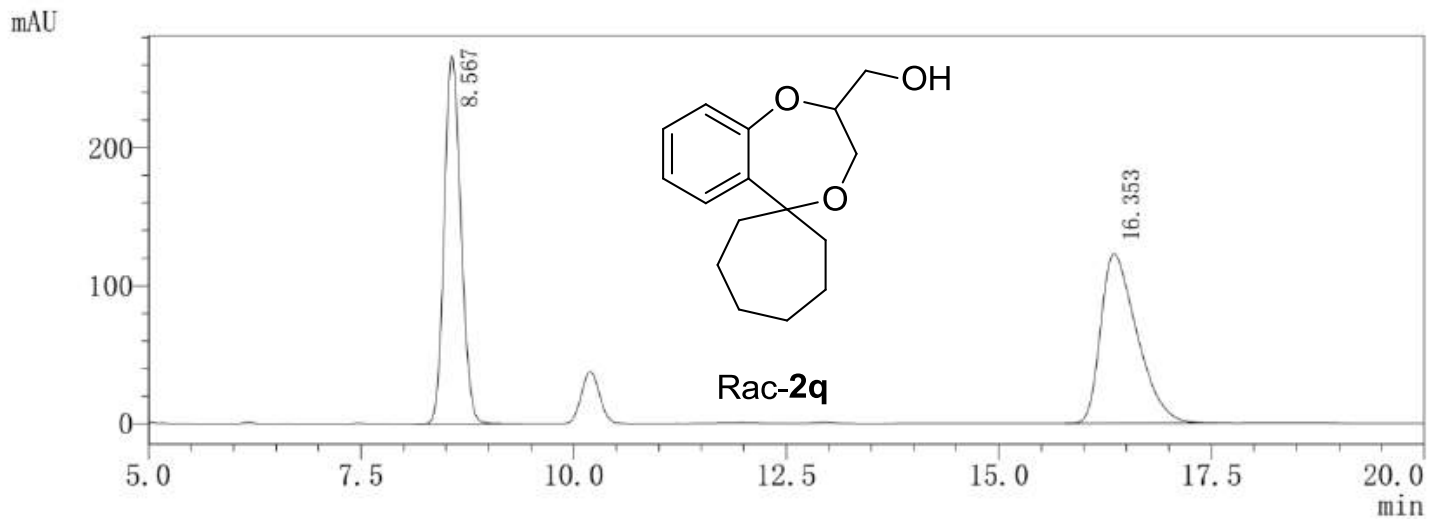

PDA Ch2 210nm 4nm

\begin{tabular}{|c|c|c|c|c|c|}
\hline Peak & Ret Time & Width & Area & Height & Area \% \\
\hline 1 & 8.567 & 0.369 & 3631132 & 266745 & 49.887 \\
\hline 2 & 16.353 & 0.788 & 3647510 & 123178 & 50.113 \\
\hline
\end{tabular}

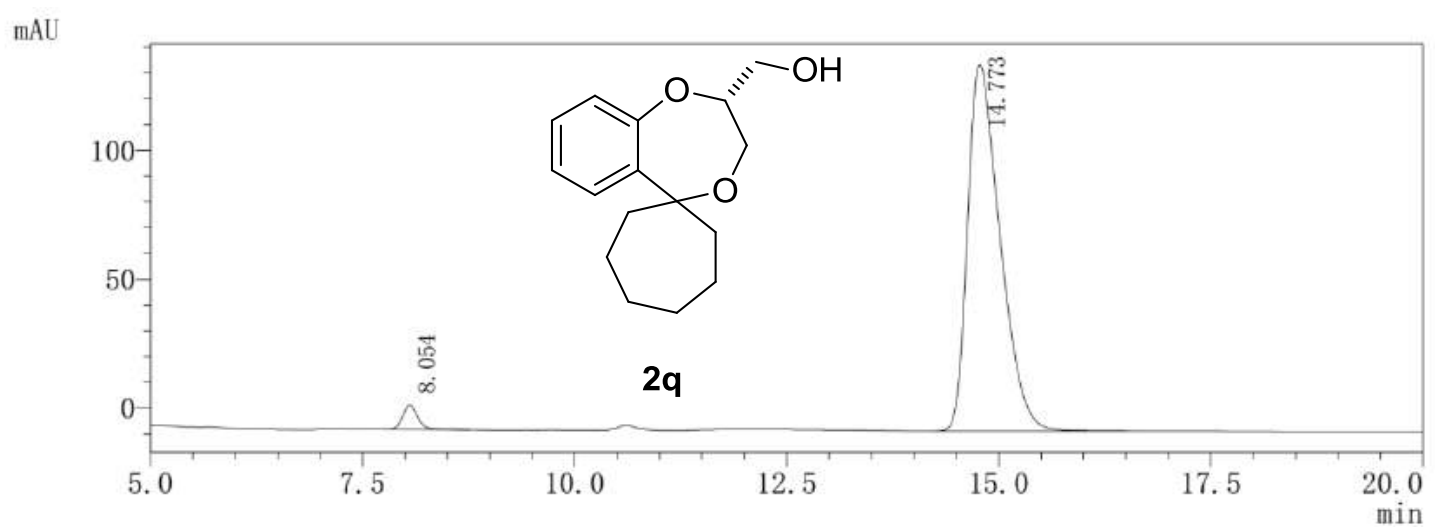

PDA Ch2 210nm 4nm

\begin{tabular}{|c|c|c|c|c|c|}
\hline Peak & Ret Time & Width & Area & Height & Area \% \\
\hline \hline 1 & 8.054 & 0.334 & 116512 & 9265 & 2.988 \\
\hline 2 & 14.773 & 0.708 & 3782706 & 142165 & 97.012 \\
\hline
\end{tabular}


$\mathrm{mAU}$

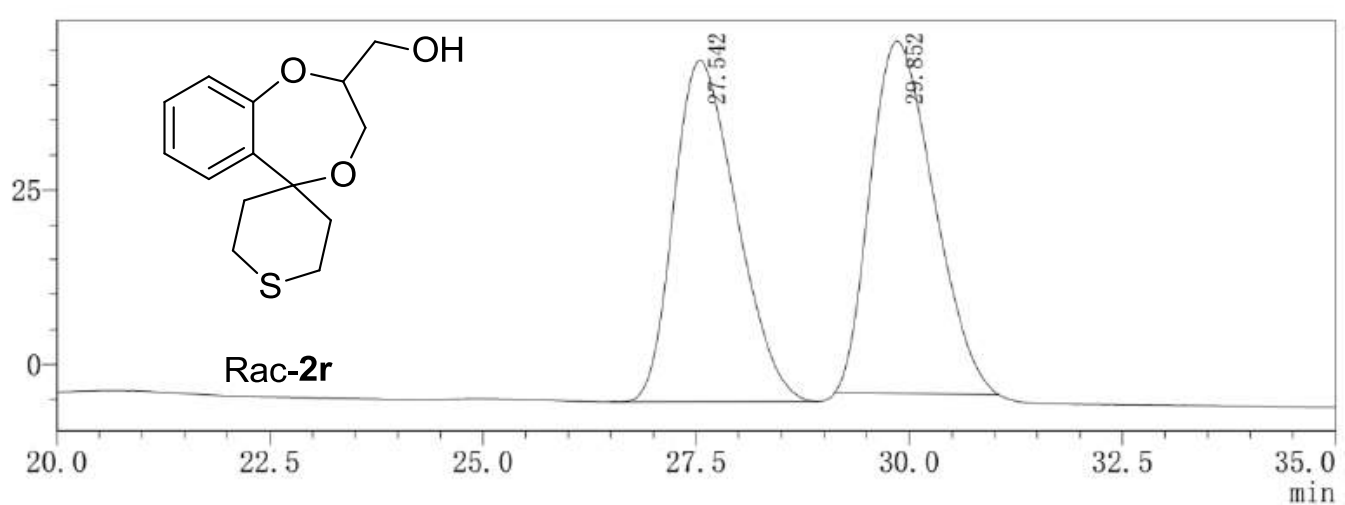

PDA Ch2 210nm 4nm

\begin{tabular}{|c|c|c|c|c|c|}
\hline Peak & Ret Time & Width & Area & Height & Area \% \\
\hline 1 & 27.542 & 1.393 & 2502913 & 48904 & 48.905 \\
\hline 2 & 29.852 & 1.418 & 2615036 & 50451 & 51.095 \\
\hline
\end{tabular}

$\mathrm{mAU}$

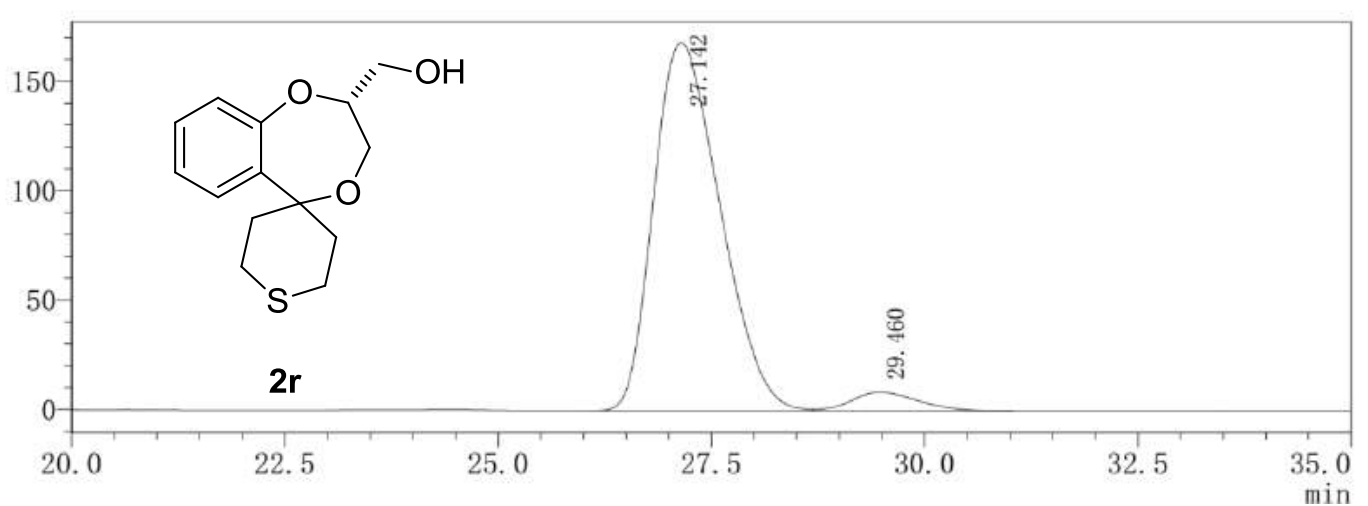

PDA Ch2 210nm 4nm

\begin{tabular}{|c|c|c|c|c|c|}
\hline Peak & Ret Time & Width & Area & Height & Area \% \\
\hline 1 & 27.142 & 1.465 & 9137474 & 168411 & 95.007 \\
\hline 2 & 29.460 & 1.487 & 480251 & 8707 & 4.993 \\
\hline
\end{tabular}




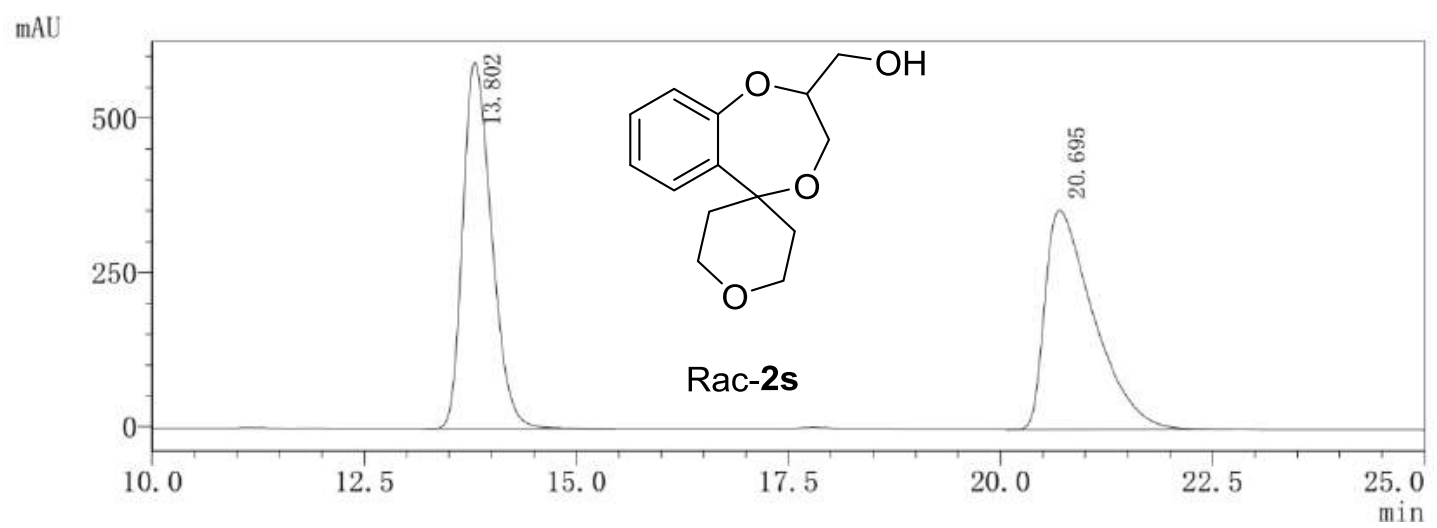

PDA Ch2 210nm 4nm

\begin{tabular}{|c|c|c|c|c|c|}
\hline Peak & Ret Time & Width & Area & Height & Area \% \\
\hline 1 & 13.802 & 0.625 & 14027712 & 593715 & 49.635 \\
\hline 2 & 20.695 & 1.055 & 14234230 & 354613 & 50.365 \\
\hline
\end{tabular}

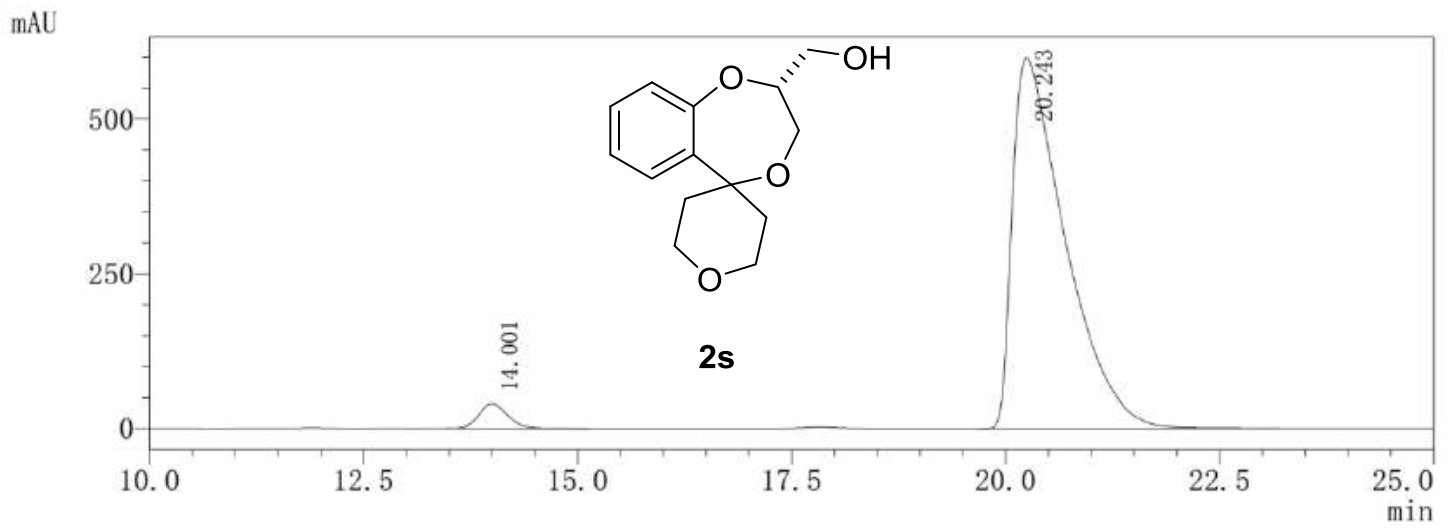

PDA Ch2 210nm 4nm

\begin{tabular}{|c|c|c|c|c|c|}
\hline Peak & Ret Time & Width & Area & Height & Area \% \\
\hline 1 & 14.001 & 0.620 & 935128 & 39957 & 3.512 \\
\hline 2 & 20.243 & 1.121 & 25693065 & 599368 & 96.488 \\
\hline
\end{tabular}




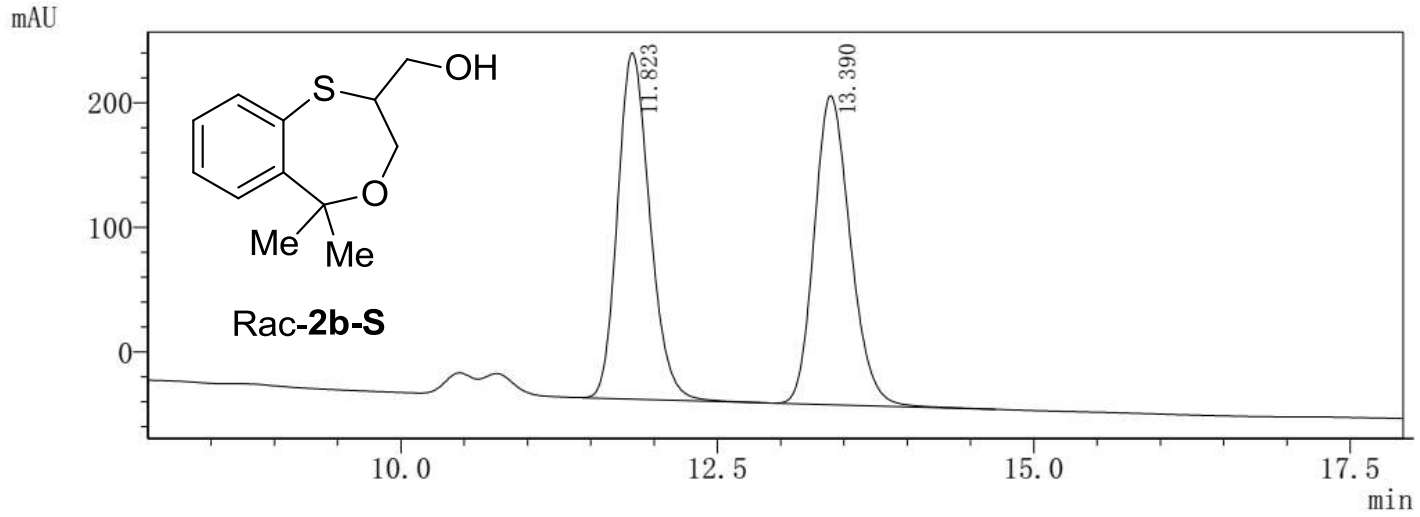

PDA Ch2 210nm 4nm

\begin{tabular}{|c|c|c|c|c|c|}
\hline Peak & Ret Time & Width & Area & Height & Area \% \\
\hline 1 & 11.823 & 0.470 & 4924345 & 278041 & 49.942 \\
\hline 2 & 13.390 & 0.527 & 4935799 & 248030 & 50.058 \\
\hline
\end{tabular}

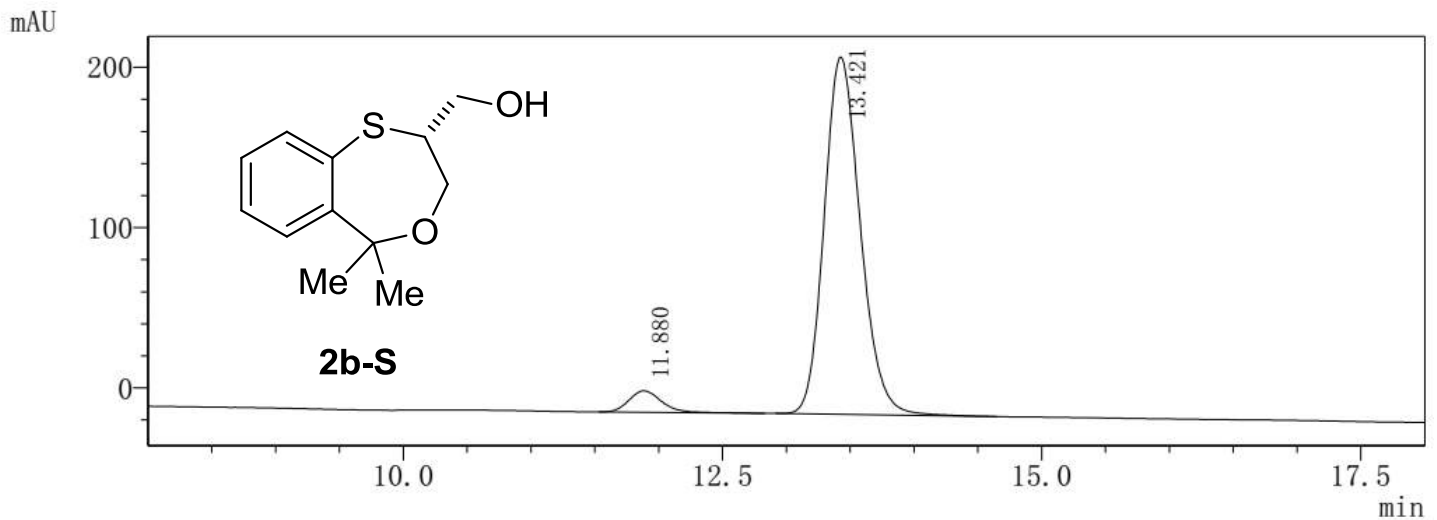

PDA Ch2 210nm 4nm

\begin{tabular}{|c|c|c|c|c|c|}
\hline Peak & Ret Time & Width & Area & Height & Area \% \\
\hline 1 & 11.880 & 0.467 & 242131 & 13459 & 5.236 \\
\hline 2 & 13.421 & 0.520 & 4382026 & 222864 & 94.764 \\
\hline
\end{tabular}




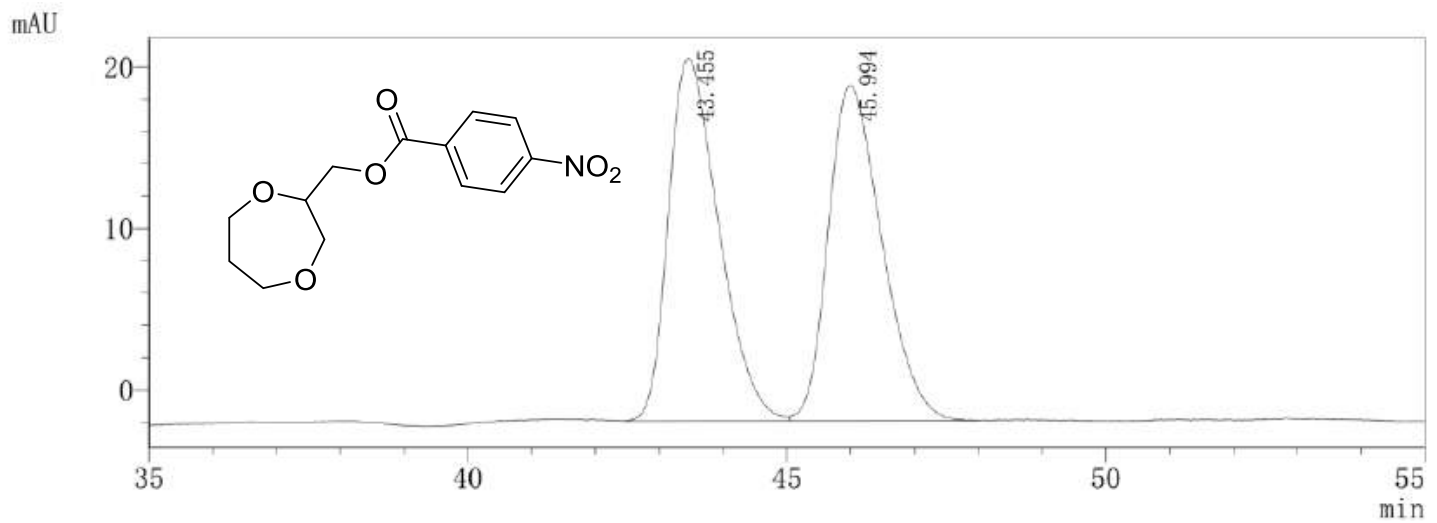

PDA Ch2 210nm 4nm

\begin{tabular}{|c|c|c|c|c|c|}
\hline Peak & Ret Time & Width & Area & Height & Area \% \\
\hline 1 & 43.455 & 1.467 & 1234955 & 22457 & 50.395 \\
\hline 2 & 45.994 & 1.554 & 1215585 & 20775 & 49.605 \\
\hline
\end{tabular}

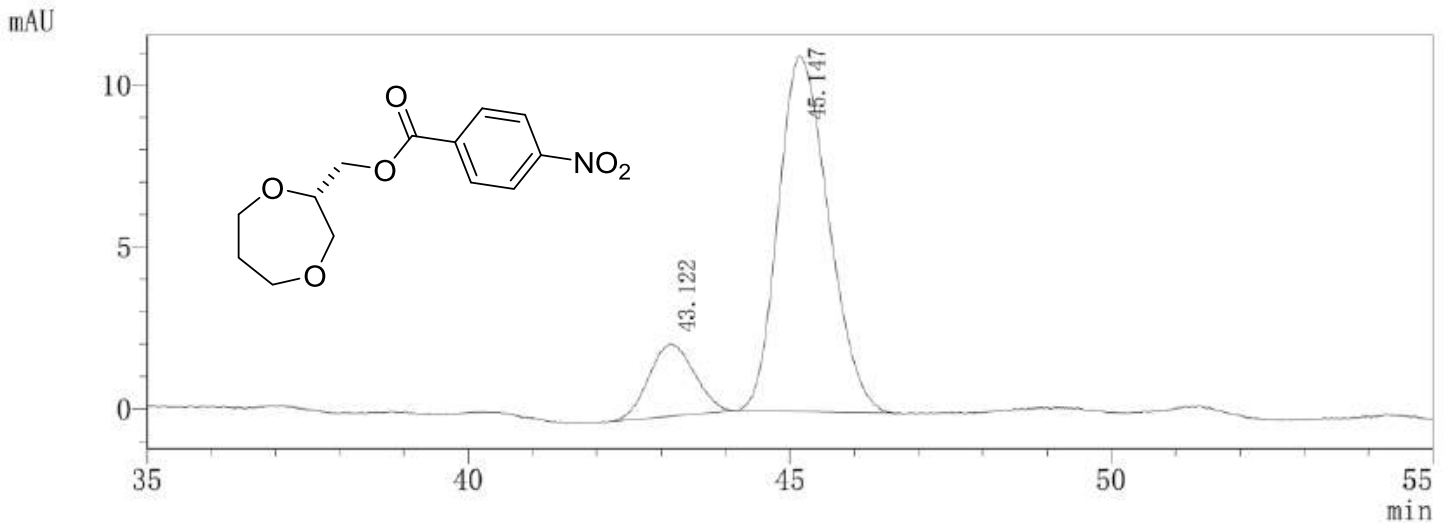

PDA Ch2 210nm 4nm

\begin{tabular}{|c|c|c|c|c|c|}
\hline Peak & Ret Time & Width & Area & Height & Area \% \\
\hline 1 & 43.122 & 1.354 & 109376 & 2233 & 15.509 \\
\hline 2 & 45.147 & 1.449 & 595847 & 10971 & 84.491 \\
\hline
\end{tabular}




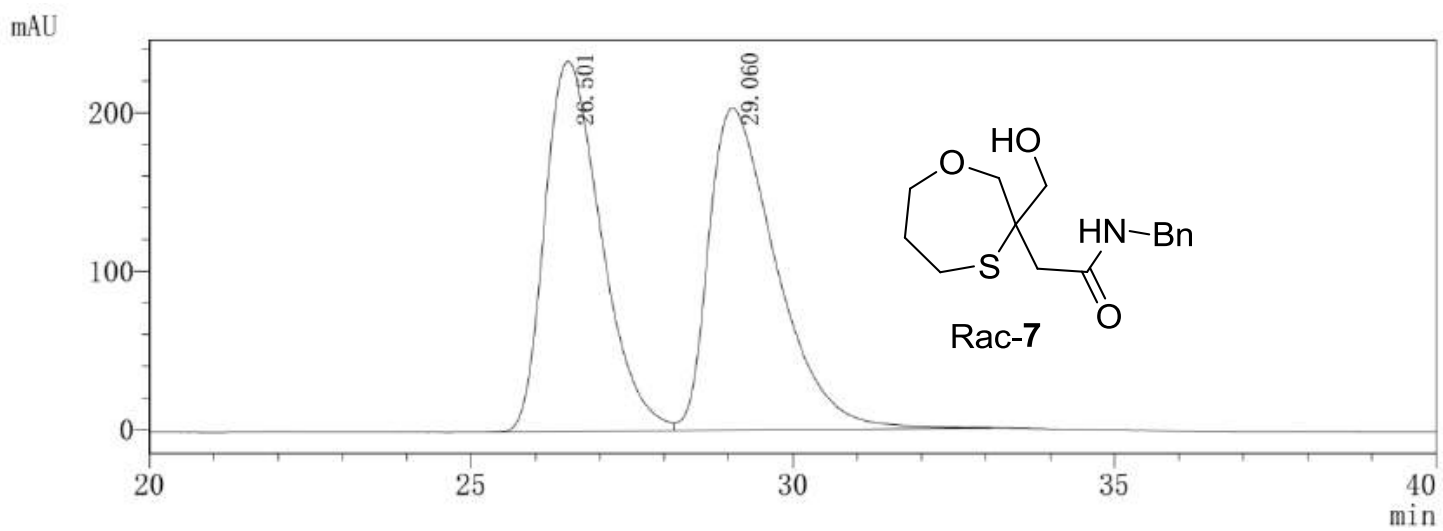

PDA Ch2 210nm 4nm

\begin{tabular}{|c|c|c|c|c|c|}
\hline Peak & Ret Time & Width & Area & Height & Area \% \\
\hline 1 & 26.501 & 1.600 & 14255855 & 233870 & 48.952 \\
\hline 2 & 29.060 & 1.886 & 14866537 & 203147 & 51.048 \\
\hline
\end{tabular}

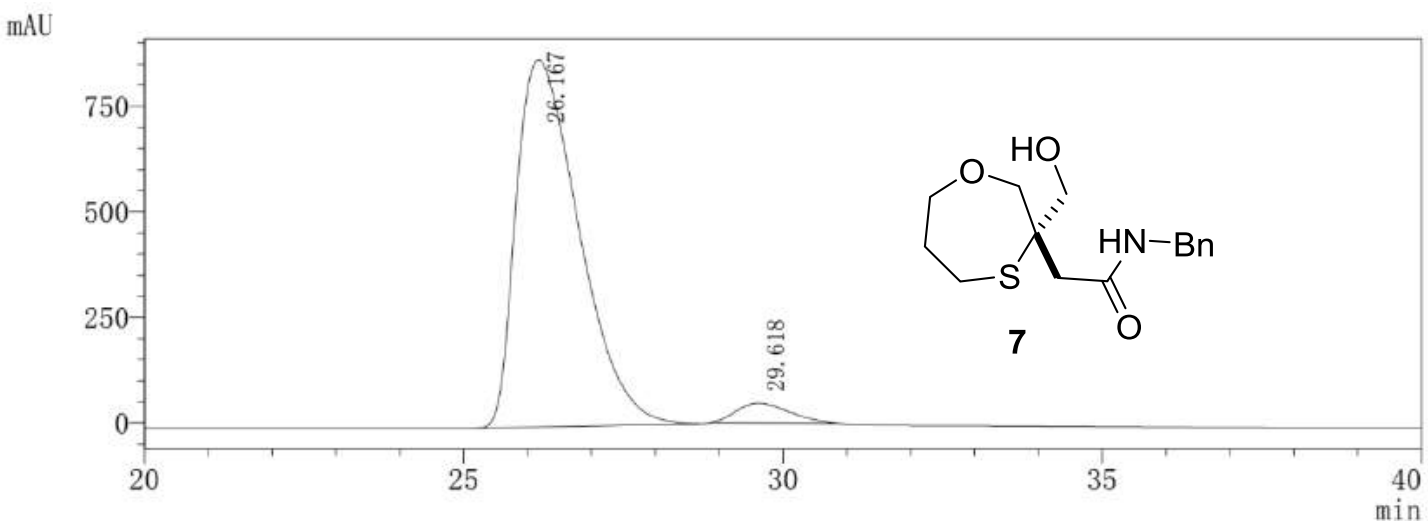

PDA Ch2 210nm 4nm

\begin{tabular}{|c|c|c|c|c|c|}
\hline Peak & Ret Time & Width & Area & Height & Area \% \\
\hline 1 & 26.167 & 1.799 & 59567098 & 870584 & 95.523 \\
\hline 2 & 29.618 & 1.611 & 2792106 & 47412 & 4.477 \\
\hline
\end{tabular}


mAU

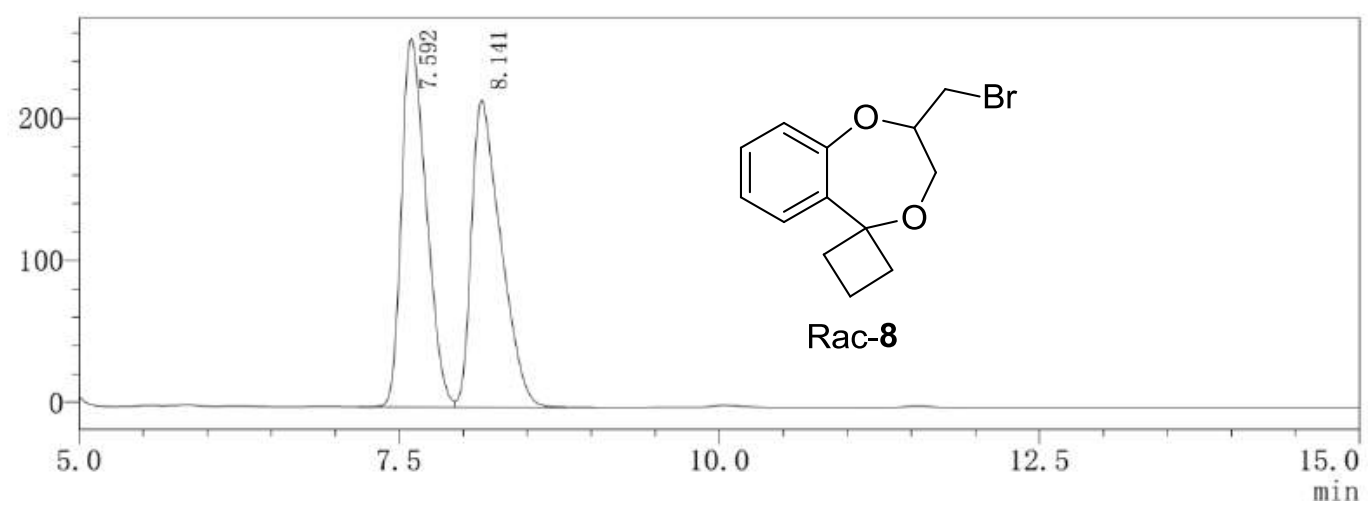

PDA Ch2 210nm 4nm

\begin{tabular}{|c|c|c|c|c|c|}
\hline Peak & Ret Time & Width & Area & Height & Area \% \\
\hline 1 & 7.592 & 0.376 & 3491038 & 259669 & 50.306 \\
\hline 2 & 8.141 & 0.442 & 3448590 & 216210 & 49.694 \\
\hline
\end{tabular}

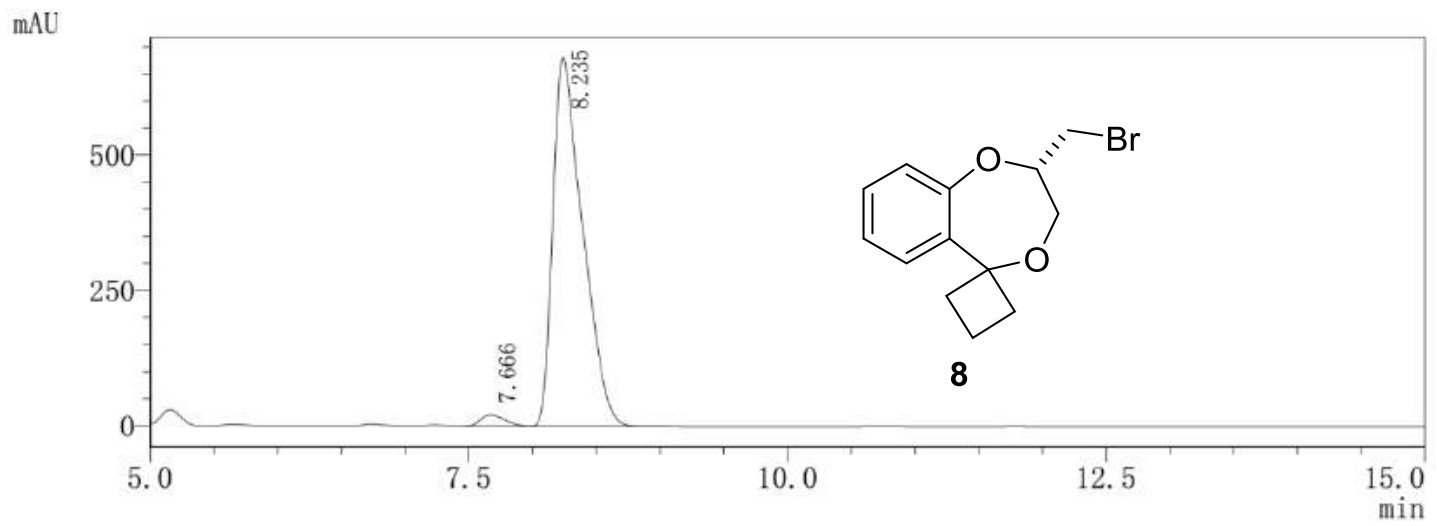

PDA Ch2 210nm 4nm

\begin{tabular}{|c|c|c|c|c|c|}
\hline Peak & Ret Time & Width & Area & Height & Area \% \\
\hline 1 & 7.666 & 0.366 & 268183 & 20786 & 2.372 \\
\hline 2 & 8.235 & 0.449 & 11038448 & 680335 & 97.628 \\
\hline
\end{tabular}




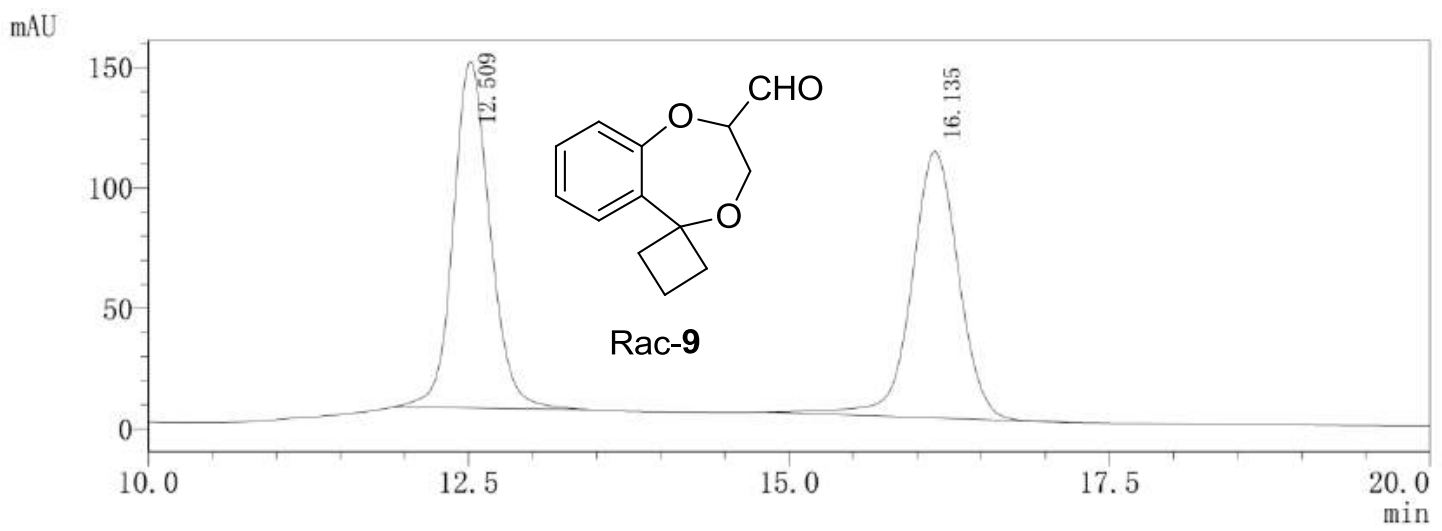

PDA Ch2 210nm 4nm

\begin{tabular}{|c|c|c|c|c|c|}
\hline Peak & Ret Time & Width & Area & Height & Area \% \\
\hline 1 & 12.509 & 0.520 & 2886129 & 143998 & 50.587 \\
\hline 2 & 16.135 & 0.656 & 2819173 & 110519 & 49.413 \\
\hline
\end{tabular}

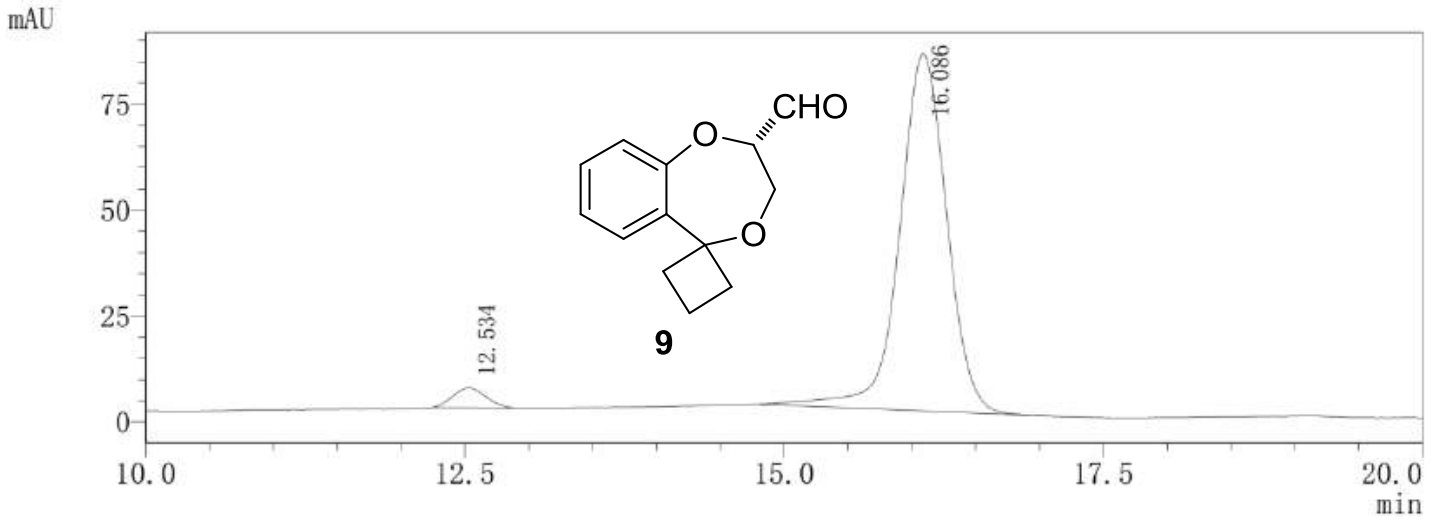

PDA Ch2 210nm 4nm

\begin{tabular}{|c|c|c|c|c|c|}
\hline Peak & Ret Time & Width & Area & Height & Area \% \\
\hline 1 & 12.534 & 0.488 & 81011 & 4552 & 3.597 \\
\hline 2 & 16.086 & 0.658 & 2171167 & 84201 & 96.403 \\
\hline
\end{tabular}




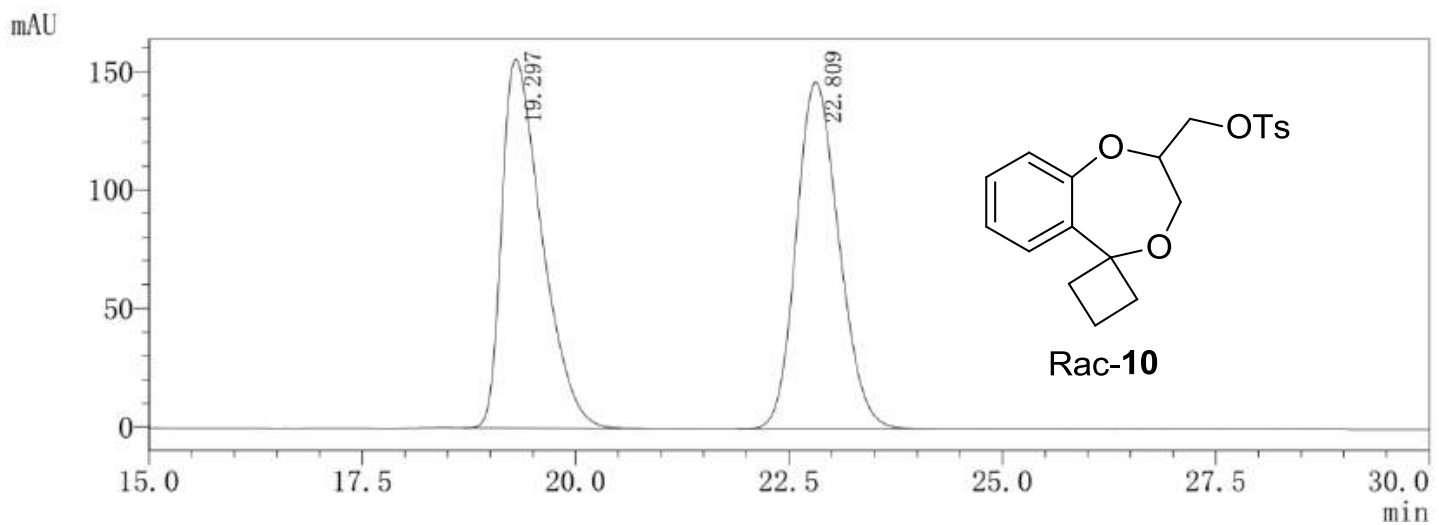

PDA Ch2 210nm 4nm

\begin{tabular}{|c|c|c|c|c|c|}
\hline Peak & Ret Time & Width & Area & Height & Area \% \\
\hline 1 & 19.297 & 0.867 & 5080723 & 155557 & 49.887 \\
\hline 2 & 22.809 & 0.928 & 5103700 & 146152 & 50.113 \\
\hline
\end{tabular}

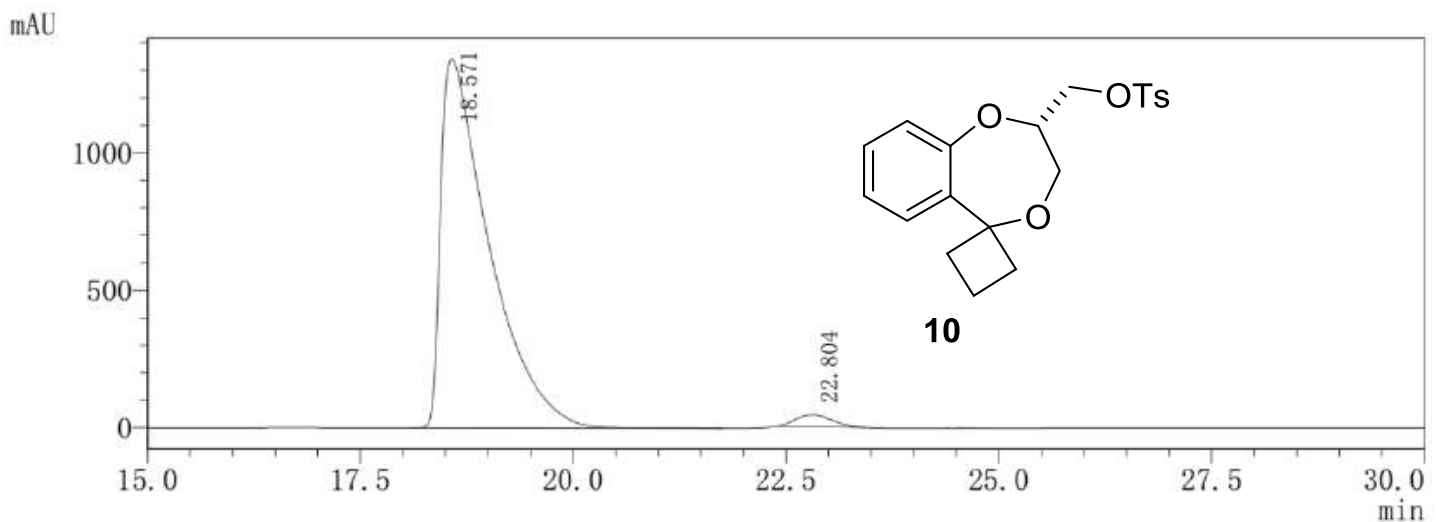

PDA Ch2 210nm 4nm

\begin{tabular}{|c|c|c|c|c|c|}
\hline Peak & Ret Time & Width & Area & Height & Area \% \\
\hline 1 & 18.571 & 1.009 & 53293080 & 1342268 & 97.597 \\
\hline 2 & 22.804 & 0.830 & 1312283 & 43436 & 2.403 \\
\hline
\end{tabular}




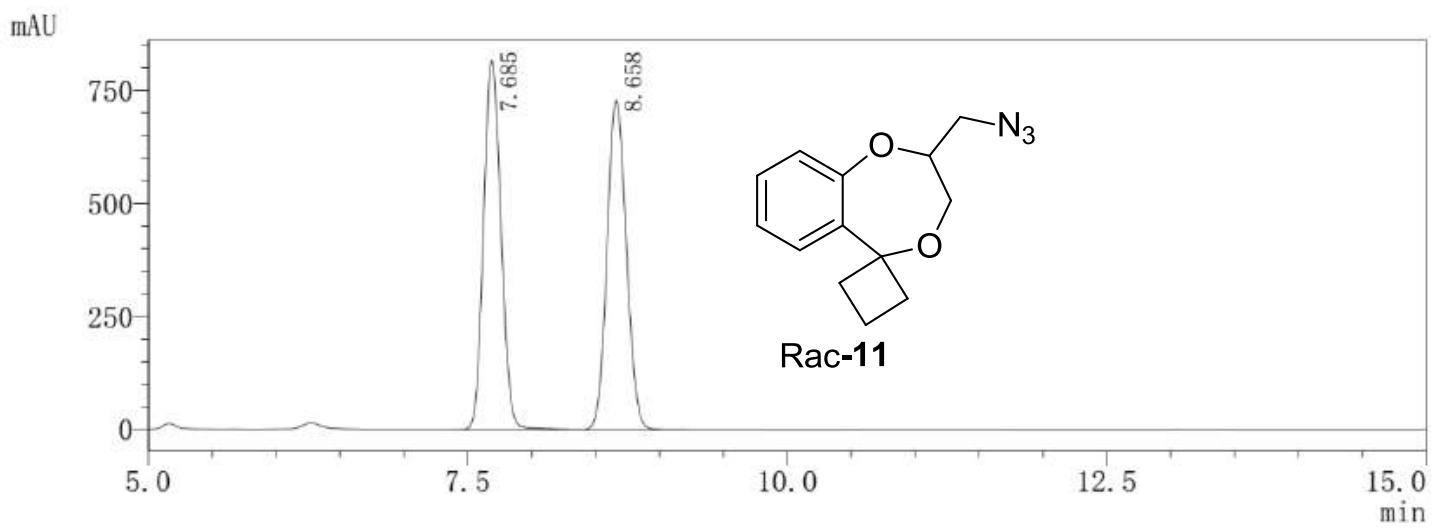

PDA Ch2 210nm 4nm

\begin{tabular}{|c|c|c|c|c|c|}
\hline Peak & Ret Time & Width & Area & Height & Area \% \\
\hline 1 & 7.685 & 0.261 & 7689908 & 816467 & 50.175 \\
\hline 2 & 8.658 & 0.291 & 7636385 & 727506 & 49.825 \\
\hline
\end{tabular}

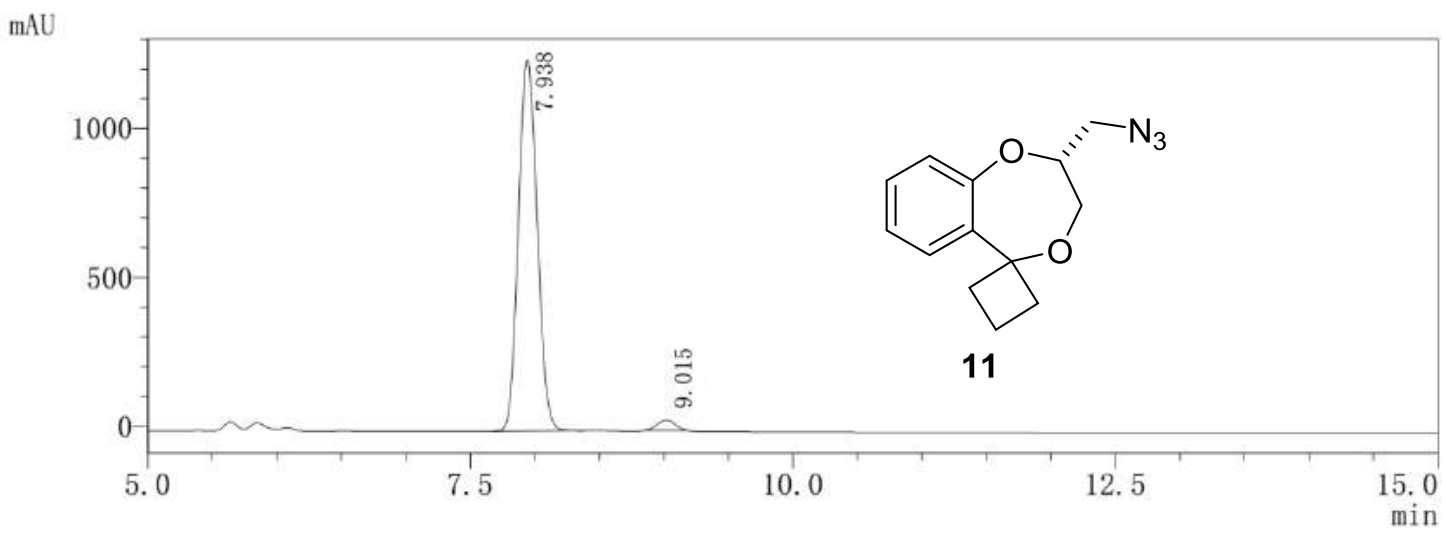

PDA Ch2 210nm 4nm

\begin{tabular}{|c|c|c|c|c|c|}
\hline Peak & Ret Time & Width & Area & Height & Area \% \\
\hline 1 & 7.938 & 0.278 & 12511967 & 1247067 & 97.382 \\
\hline 2 & 9.015 & 0.282 & 336341 & 33890 & 2.618 \\
\hline
\end{tabular}




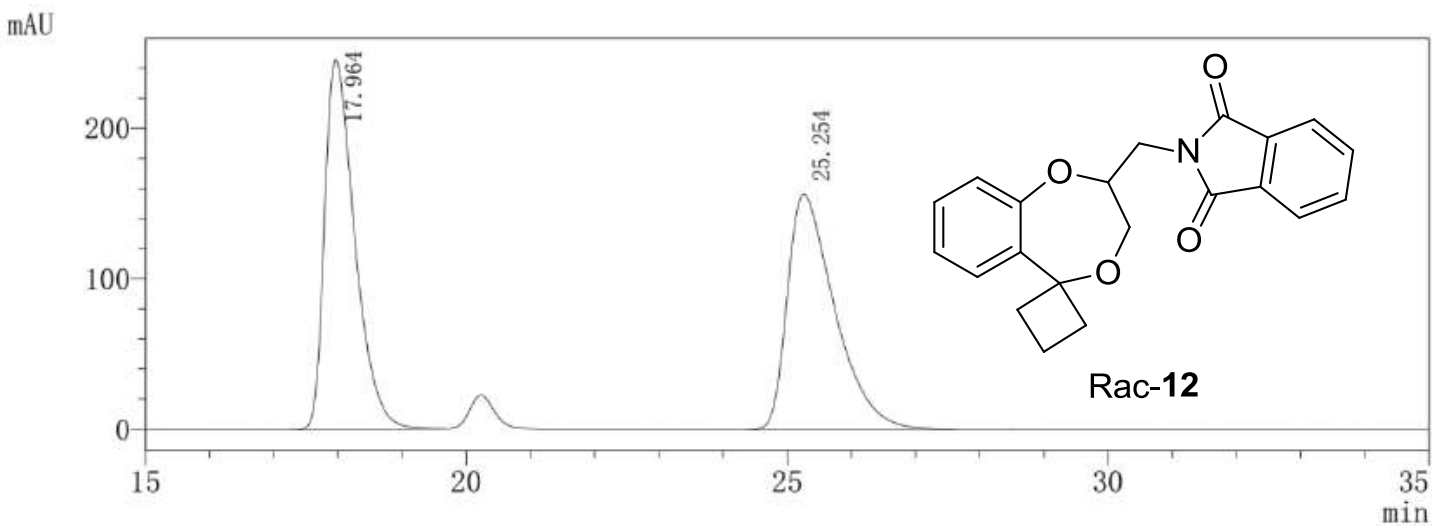

PDA Ch2 210nm 4nm

\begin{tabular}{|c|c|c|c|c|c|}
\hline Peak & Ret Time & Width & Area & Height & Area \% \\
\hline 1 & 17.964 & 0.853 & 8033790 & 246019 & 49.980 \\
\hline 2 & 25.254 & 1.333 & 8040237 & 156711 & 50.020 \\
\hline
\end{tabular}

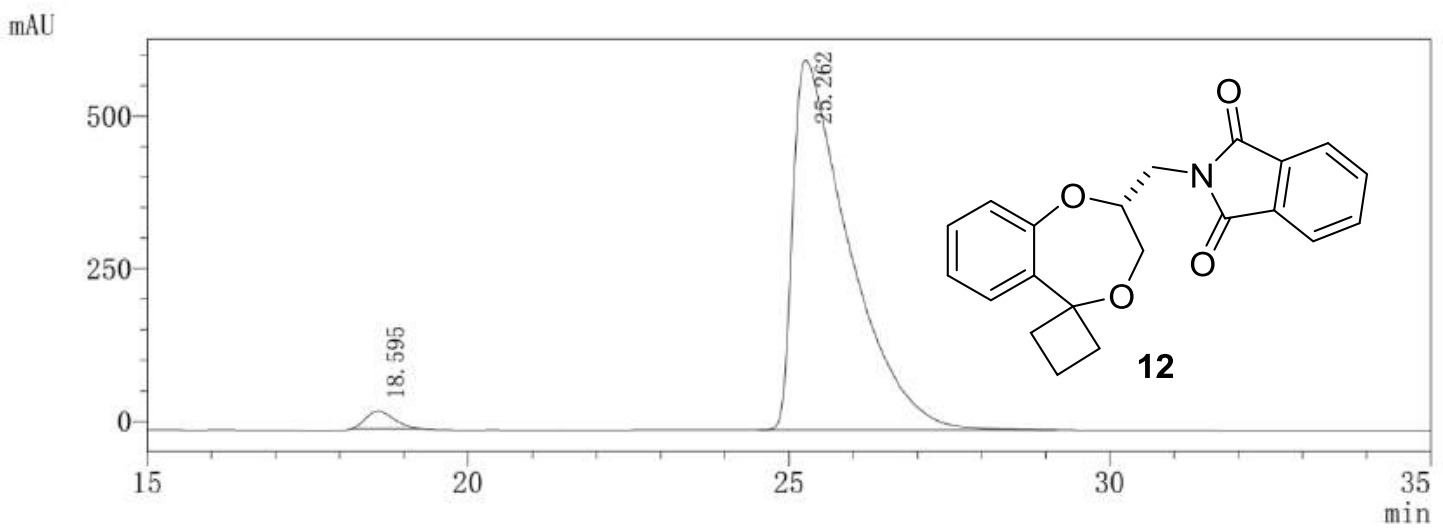

PDA Ch2 210nm 4nm

\begin{tabular}{|c|c|c|c|c|c|}
\hline Peak & Ret Time & Width & Area & Height & Area \% \\
\hline 1 & 18.595 & 0.851 & 910045 & 29275 & 2.402 \\
\hline 2 & 25.262 & 1.561 & 36973304 & 606340 & 97.598 \\
\hline
\end{tabular}




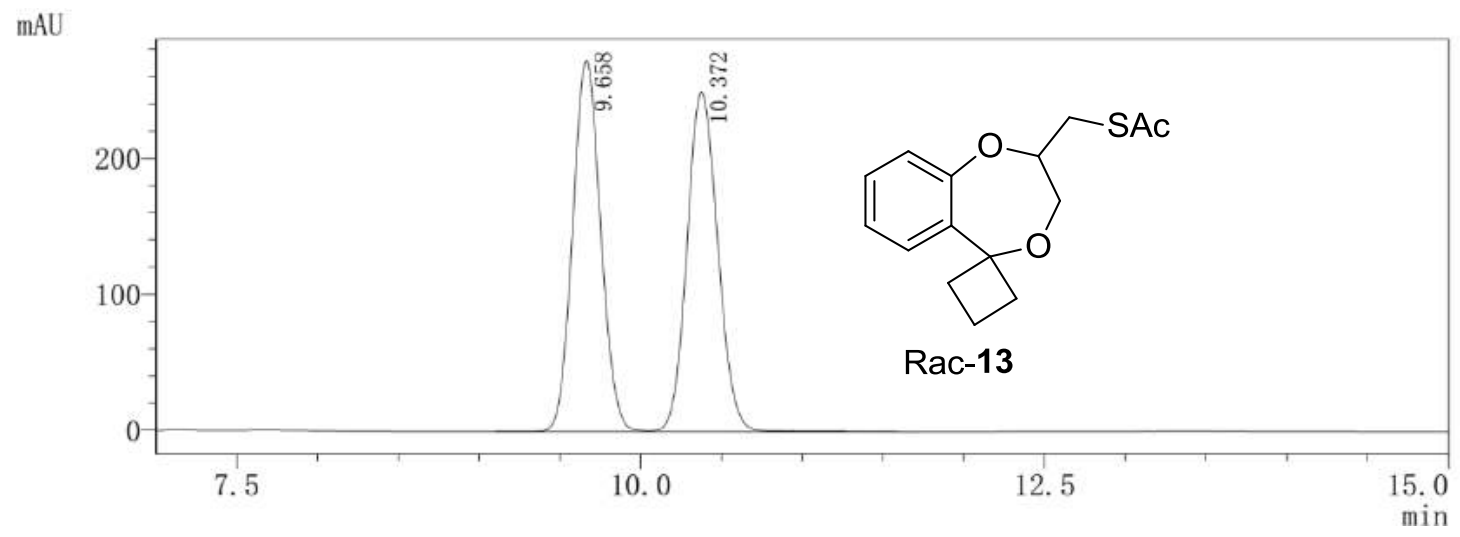

PDA Ch2 210nm 4nm

\begin{tabular}{|c|c|c|c|c|c|}
\hline Peak & Ret Time & Width & Area & Height & Area \% \\
\hline 1 & 9.658 & 0.330 & 3296692 & 273234 & 49.828 \\
\hline 2 & 10.372 & 0.360 & 3319455 & 250020 & 50.172 \\
\hline
\end{tabular}

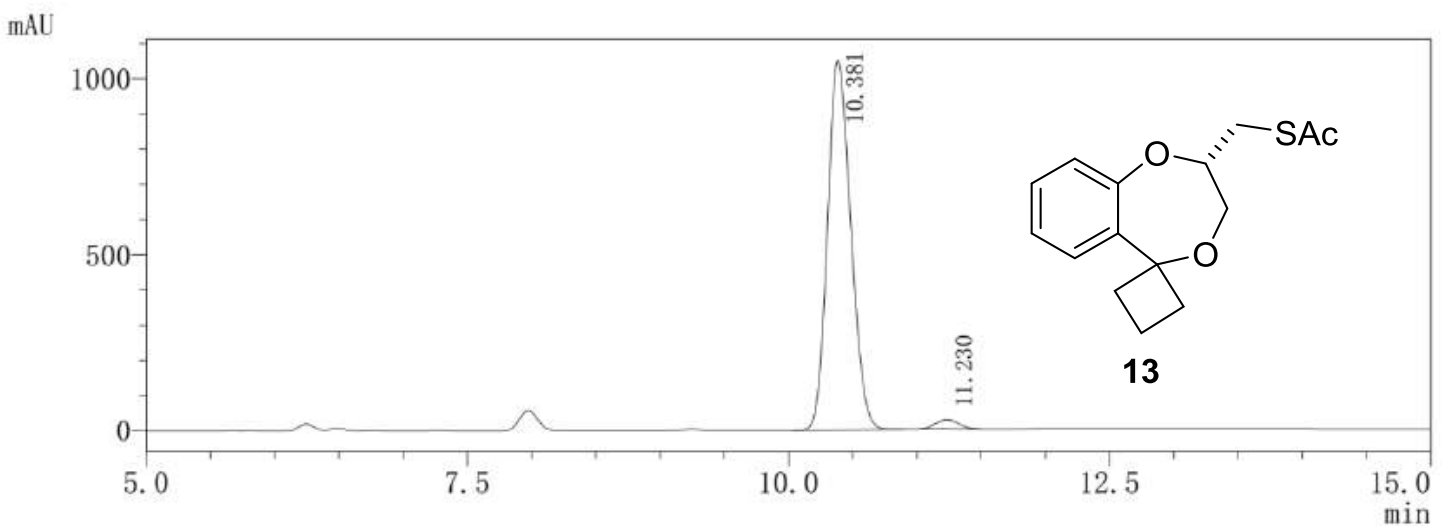

PDA Ch2 210nm 4nm

\begin{tabular}{|c|c|c|c|c|c|}
\hline Peak & Ret Time & Width & Area & Height & Area \% \\
\hline 1 & 10.381 & 0.348 & 13329860 & 1051610 & 97.508 \\
\hline 2 & 11.230 & 0.356 & 340705 & 26548 & 2.492 \\
\hline
\end{tabular}




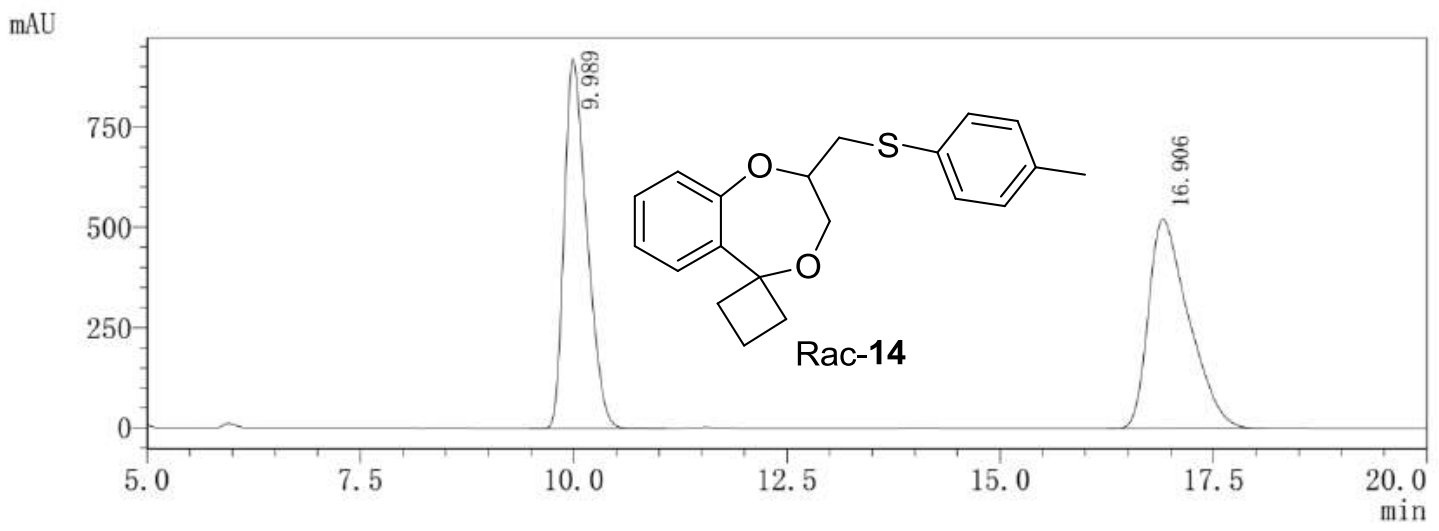

PDA Ch2 210nm 4nm

\begin{tabular}{|c|c|c|c|c|c|}
\hline Peak & Ret Time & Width & Area & Height & Area \% \\
\hline 1 & 9.989 & 0.509 & 17074969 & 921268 & 50.026 \\
\hline 2 & 16.906 & 0.877 & 17057466 & 519708 & 49.974 \\
\hline
\end{tabular}

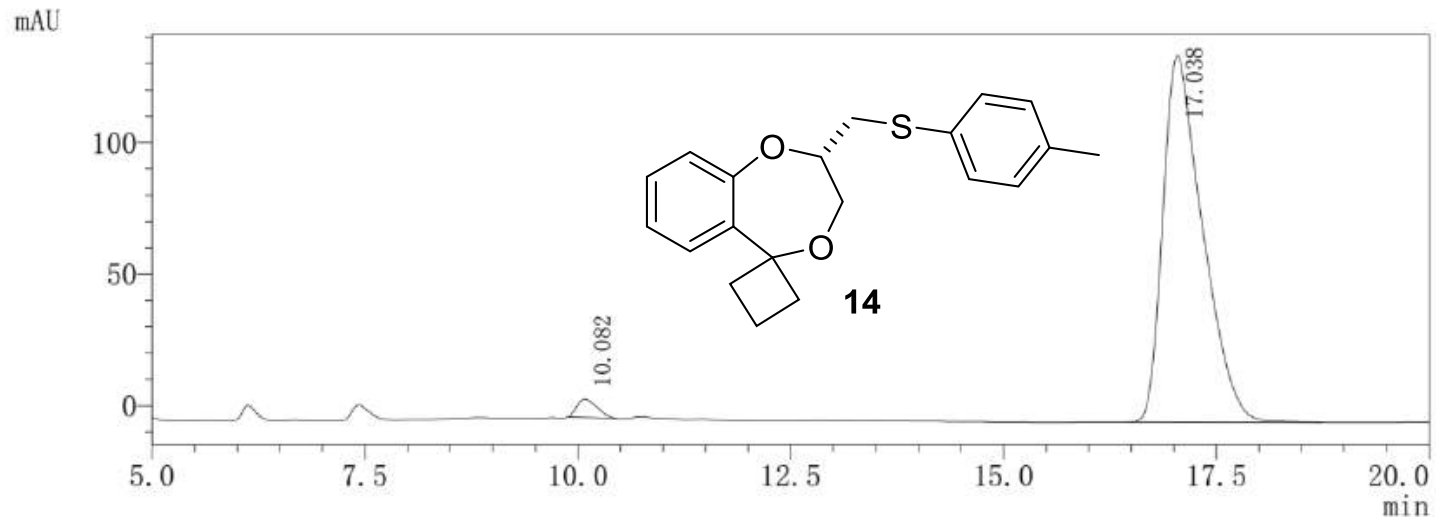

PDA Ch2 210nm 4nm

\begin{tabular}{|c|c|c|c|c|c|}
\hline Peak & Ret Time & Width & Area & Height & Area \% \\
\hline 1 & 10.082 & 0.462 & 111723 & 6868 & 2.406 \\
\hline 2 & 17.038 & 0.863 & 4531316 & 139479 & 97.594 \\
\hline
\end{tabular}




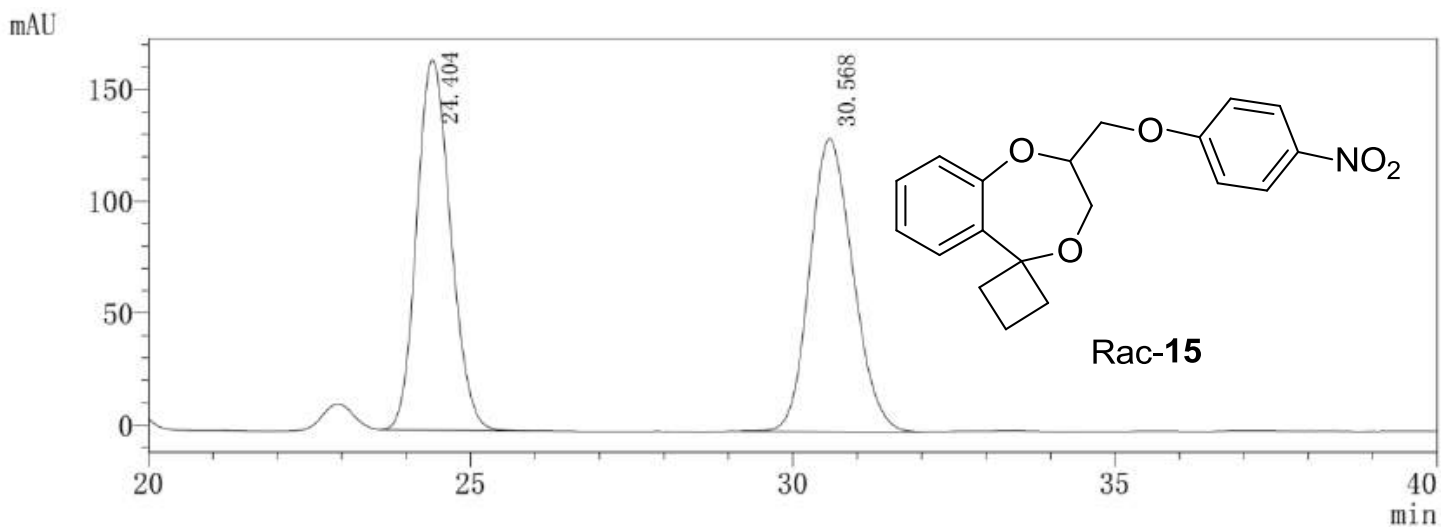

PDA Ch2 210nm 4nm

\begin{tabular}{|c|c|c|c|c|c|}
\hline Peak & Ret Time & Width & Area & Height & Area \% \\
\hline 1 & 24.404 & 0.973 & 6049410 & 165489 & 49.816 \\
\hline 2 & 30.568 & 1.237 & 6094009 & 130670 & 50.184 \\
\hline
\end{tabular}

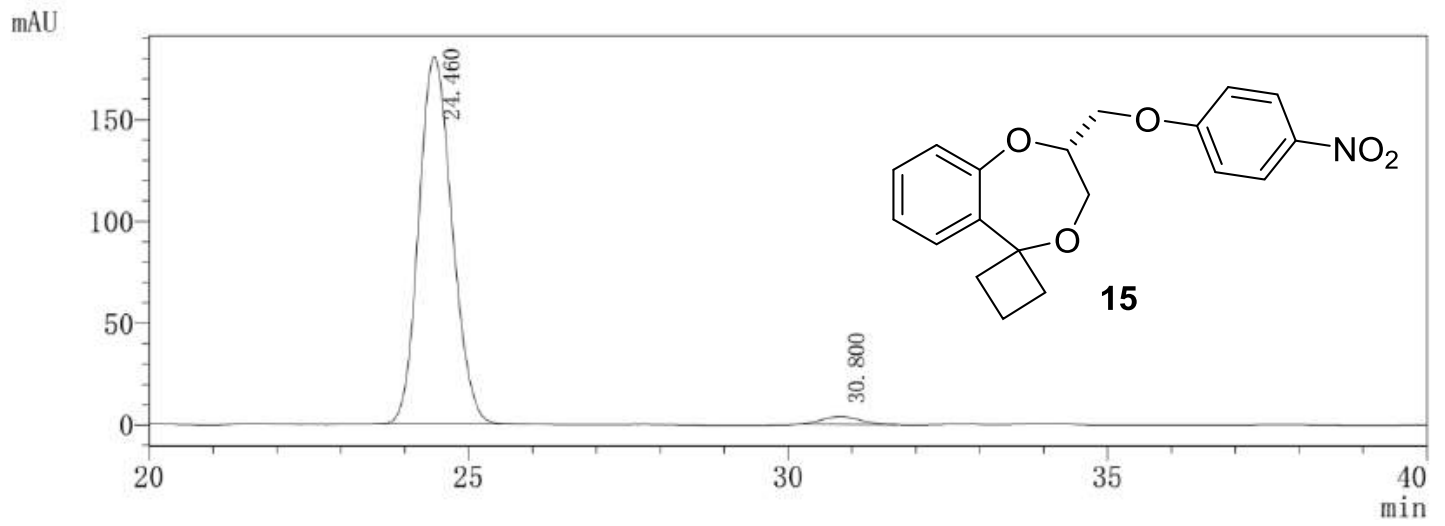

PDA Ch2 210nm 4nm

\begin{tabular}{|c|c|c|c|c|c|}
\hline Peak & Ret Time & Width & Area & Height & Area \% \\
\hline 1 & 24.460 & 0.967 & 6565804 & 180560 & 97.439 \\
\hline 2 & 30.800 & 1.172 & 172570 & 4020 & 2.561 \\
\hline
\end{tabular}

